\title{
THE FOURIER TRANSFORM FOR CERTAIN HYPERKÄHLER FOURFOLDS
}

\author{
MINGMIN SHEN AND CHARLES VIAL
}

\begin{abstract}
Using a codimension-1 algebraic cycle obtained from the Poincaré line bundle, Beauville defined the Fourier transform on the Chow groups of an abelian variety $A$ and showed that the Fourier transform induces a decomposition of the Chow ring $\mathrm{CH}^{*}(A)$. By using a codimension-2 algebraic cycle representing the Beauville-Bogomolov class, we give evidence for the existence of a similar decomposition for the Chow ring of hyperkähler varieties deformation equivalent to the Hilbert scheme of length-2 subschemes on a K3 surface. We indeed establish the existence of such a decomposition for the Hilbert scheme of length-2 subschemes on a K3 surface and for the variety of lines on a very general cubic fourfold.
\end{abstract}

\section{CONTEnTs}

Introduction

Part 1. The Fourier transform for hyperkähler fourfolds 13

1. The cohomological Fourier transform $\quad 13$

2. The Fourier transform on the Chow groups of hyperkähler fourfolds $\quad 17$

3. The Fourier decomposition is motivic 22

4. First multiplicative results 25

5. An application to symplectic automorphisms 27

6. On the birational invariance of the Fourier decomposition 28

7. An alternate approach to the Fourier decomposition on the Chow ring of abelian varieties 30

8. Multiplicative Chow-Künneth decompositions 33

9. Algebraicity of $\mathfrak{B}$ for hyperkähler varieties of $\mathrm{K} 3^{[n]}$-type $\quad 39$

Part 2. The Hilbert scheme $S^{[2]}$

10. Basics on the Hilbert scheme of length-2 subschemes on a variety $X$

11. The incidence correspondence $I \quad 46$

12. Decomposition results on the Chow groups of $X^{[2]} \quad 51$

13. Multiplicative Chow-Künneth decomposition for $X^{[2]}$

14. The Fourier decomposition for $S^{[2]} \quad 63$

15. The Fourier decomposition for $S^{[2]}$ is multiplicative $\quad 69$

16. The cycle $L$ of $S^{[2]}$ via moduli of stable sheaves 74

Part 3. The variety of lines on a cubic fourfold $\quad 76$

17. The incidence correspondence $I \quad 76$

18. The rational self-map $\varphi: F \rightarrow F \quad 78$

19. The Fourier decomposition for $F \quad 80$

20. A first multiplicative result $\quad 82$

21. The rational self-map $\varphi: F \rightarrow F$ and the Fourier decomposition 86

22. The Fourier decomposition for $F$ is multiplicative 94

Appendix A. Some geometry of cubic fourfolds 96

Appendix B. Rational maps and Chow groups 100

References $\quad 103$

Date: May 2, 2014.

2010 Mathematics Subject Classification. 14C25, 14C15, 53C26, 14J28, 14J32, 14K99, 14C17.

Key words and phrases. Hyperkähler manifolds, Irreducible holomorphic symplectic varieties, Cubic fourfolds, Fano schemes of lines, K3 surfaces, Hilbert schemes of points, Abelian varieties, Motives, Algebraic cycles, Chow groups, Chow ring, Chow-Künneth decomposition, Bloch-Beilinson filtration, Modified diagonals.

The first author is supported by the Simons Foundation as a Simons Postdoctoral Fellow.

The second author is supported by EPSRC Early Career Fellowship number EP/K005545/1. 


\section{INTRODUCTION}

A. Abelian varieties. Let $A$ be an abelian variety of dimension $d$ over a field $k$. Let $\hat{A}=\operatorname{Pic}^{0}(A)$ be its dual and let $L$ be the Poincaré line bundle on $A \times \hat{A}$ viewed as an element of $\mathrm{CH}^{1}(A \times \hat{A})$. The Fourier transform on the Chow groups with rational coefficients is defined as

$$
\mathcal{F}(\sigma):=p_{2, *}\left(e^{L} \cdot p_{1}^{*} \sigma\right), \quad \text { for all } \sigma \in \mathrm{CH}^{i}(A) .
$$

Here, $e^{L}:=[A \times \hat{A}]+L+\frac{L^{2}}{2 !}+\ldots+\frac{L^{2 d}}{(2 d) !}$, and $p_{1}: A \times \hat{A} \rightarrow A$ and $p_{2}: A \times \hat{A} \rightarrow \hat{A}$ are the two projections. The main result of $[8]$ is the following.

Theorem (Beauville). Let $A$ be an abelian variety of dimension d. The Fourier transform induces a canonical splitting

$$
\mathrm{CH}^{i}(A)=\bigoplus_{s=i-d}^{i} \mathrm{CH}^{i}(A)_{s}, \quad \text { where } \mathrm{CH}^{i}(A)_{s}:=\left\{\sigma \in \mathrm{CH}^{i}(A): \mathcal{F}(\sigma) \in \mathrm{CH}^{d-i+s}(\hat{A})\right\} .
$$

Furthermore, this decomposition enjoys the following two properties:

(a) $\mathrm{CH}^{i}(A)_{s}=\left\{\sigma \in \mathrm{CH}^{i}(A):[n]^{*} \sigma=n^{2 i-s} \sigma\right\}$, where $[n]: A \rightarrow A$ is the multiplication-by-n map ;

(b) $\mathrm{CH}^{i}(A)_{s} \cdot \mathrm{CH}^{j}(A)_{r} \subseteq \mathrm{CH}^{i+j}(A)_{r+s}$.

Property (a) shows that the Fourier decomposition (1) is canonical, while Property (b) shows that the Fourier decomposition is compatible with the ring structure on $\mathrm{CH}^{*}(A)$ given by intersection product. It should be mentioned that, as explained in [8], (1) is expected to be the splitting of a Bloch-Beilinson type filtration on $\mathrm{CH}^{*}(A)$. By [21], this splitting is in fact induced by a Chow-Künneth decomposition of the diagonal and it is of Bloch-Beilinson type if it satisfies the following two properties :

(B) $\mathrm{CH}^{i}(A)_{s}=0$ for all $s<0$;

(D) the cycle class map $\mathrm{CH}^{i}(A)_{0} \rightarrow \mathrm{H}^{2 i}(A, \mathbb{Q})$ is injective for all $i$.

Actually, if Property (D) is true for all abelian varieties, then Property (B) is true for all abelian varieties ; see [51].

A direct consequence of the Fourier decomposition on the Chow ring of an abelian variety is the following. First note that $\mathrm{CH}^{d}(A)_{0}=\langle[0]\rangle$, where [0] is the class of the identity element $0 \in A$. Let $D_{1}, \ldots, D_{d}$ be symmetric divisors, that is, divisors such that $[-1]^{*} D_{i}=D_{i}$, or equivalently $D_{i} \in \mathrm{CH}^{1}(A)_{0}$, for all $i$. Then

$$
D_{1} \cdot D_{2} \cdot \ldots \cdot D_{d}=\operatorname{deg}\left(D_{1} \cdot D_{2} \cdot \ldots \cdot D_{d}\right)[0] \quad \text { in } \mathrm{CH}^{d}(A) .
$$

So far, for lack of a Bloch-Beilinson type filtration on the Chow groups of hyperkähler varieties, it is this consequence (2), and variants thereof, of the canonical splitting of the Chow ring of an abelian variety that have been tested for certain types of hyperkähler varieties. Property (2) for divisors on hyperkähler varieties $F$ is Beauville's weak splitting conjecture [9]; it was subsequently strengthened in [54] to include the Chern classes of $F$. The goal of this manuscript is to show that a Fourier decomposition should exist on the Chow ring of hyperkähler fourfolds which are deformation equivalent to the Hilbert scheme of length-2 subschemes on a K3 surface. Before making this more precise, we first consider the case of K3 surfaces.

B. K3 surfaces. Let $S$ be a complex projective K3 surface. In that case, a Bloch-Beilinson type filtration on $\mathrm{CH}^{*}(S)$ is explicit : we have $\mathrm{F}^{1} \mathrm{CH}^{2}(S)=\mathrm{F}^{2} \mathrm{CH}^{2}(S)=\mathrm{CH}^{2}(S)_{\text {hom }}:=\operatorname{ker}\left\{c l: \mathrm{CH}^{2}(S) \rightarrow \mathrm{H}^{4}(S, \mathbb{Q})\right\}$ and $\mathrm{F}^{1} \mathrm{CH}^{1}(S)=0$. Beauville and Voisin [11] observed that this filtration splits canonically by showing the existence of a zero-cycle $\mathfrak{o}_{S} \in \mathrm{CH}^{2}(S)$, which is the class of any point lying on a rational curve on $S$, such that the intersection of any two divisors on $S$ is proportional to $\mathfrak{o}_{S}$. Let us introduce the Chow-Künneth decomposition

$$
\pi_{S}^{0}:=\mathfrak{o}_{S} \times S, \quad \pi_{S}^{4}:=S \times \mathfrak{o}_{S} \quad \text { and } \quad \pi_{S}^{2}:=\Delta_{S}-\mathfrak{o}_{S} \times S-S \times \mathfrak{o}_{S} \quad \text { in } \mathrm{CH}^{2}(S \times S) .
$$

Cohomologically, $\pi_{S}^{0}, \pi_{S}^{2}$ and $\pi_{S}^{4}$ are the Künneth projectors on $\mathrm{H}^{0}(S, \mathbb{Q}), \mathrm{H}^{2}(S, \mathbb{Q})$, and $\mathrm{H}^{4}(S, \mathbb{Q})$, respectively. The result of Beauville-Voisin shows that among the Chow-Künneth decompositions of $\Delta_{S}$ (note that a pair of zero-cycles of degree 1 induces a Chow-Künneth decomposition for $S$ and two distinct pairs 
induce distinct such decompositions) the symmetric one associated to $\mathfrak{o}_{S}$, denoted $\Delta_{S}=\pi_{S}^{0}+\pi_{S}^{2}+\pi_{S}^{4}$, is special because the decomposition it induces on the Chow groups of $S$ is compatible with the ring structure on $\mathrm{CH}^{*}(S)$.

A key property satisfied by $\mathfrak{o}_{S}$, proved in [11], is that $c_{2}(S)=24 \mathfrak{o}_{S}$. Let $\iota_{\Delta}: S \rightarrow S \times S$ be the diagonal embedding. Having in mind that the top Chern class of the tangent bundle $c_{2}(S)$ is equal to $\iota_{\Delta}^{*} \Delta_{S}$, we see that the self-intersection of the Chow-Künneth projector $\pi_{S}^{2}$ satisfies

$$
\begin{aligned}
\pi_{S}^{2} \cdot \pi_{S}^{2} & =\Delta_{S}^{\cdot 2}-2 \Delta_{S} \cdot\left(\mathfrak{o}_{S} \times S+S \times \mathfrak{o}_{S}\right)+\left(\mathfrak{o}_{S} \times S+S \times \mathfrak{o}_{S}\right)^{\cdot 2} \\
& =\left(\iota_{\Delta}\right)_{*} c_{2}(S)-2 \mathfrak{o}_{S} \times \mathfrak{o}_{S} \\
& =22 \mathfrak{o}_{S} \times \mathfrak{o}_{S} .
\end{aligned}
$$

We then observe that the action of $e^{\pi_{S}^{2}}:=S \times S+\pi_{S}^{2}+\frac{1}{2} \pi_{S}^{2} \cdot \pi_{S}^{2}=S \times S+\pi_{S}^{2}+11 \mathfrak{o}_{S} \times \mathfrak{o}_{S}$ on the Chow group $\mathrm{CH}^{*}(S)$, called the Fourier transform and denoted $\mathcal{F}$, induces the same splitting as the Chow-Künneth decomposition considered above. Indeed, writing

$$
\mathrm{CH}^{i}(S)_{s}:=\left\{\sigma \in \mathrm{CH}^{i}(S): \mathcal{F}(\sigma) \in \mathrm{CH}^{2-i+s}(S)\right\},
$$

we also have

$$
\mathrm{CH}^{i}(S)_{s}=\left(\pi_{S}^{2 i-s}\right)_{*} \mathrm{CH}^{i}(S) .
$$

With these notations, we then have $\mathrm{CH}^{2}(S)=\mathrm{CH}^{2}(S)_{0} \oplus \mathrm{CH}^{2}(S)_{2}, \mathrm{CH}^{1}(S)=\mathrm{CH}^{1}(S)_{0}$ and $\mathrm{CH}^{0}(S)=$ $\mathrm{CH}^{0}(S)_{0}$, with the multiplicative property that

$$
\mathrm{CH}^{2}(S)_{0}=\mathrm{CH}^{1}(S)_{0} \cdot \mathrm{CH}^{1}(S)_{0}=\left\langle\mathfrak{o}_{S}\right\rangle .
$$

The Beauville-Bogomolov form $q_{S}$ on a K3 surface $S$ is simply given by the cup-product on $\mathrm{H}^{2}(S, \mathbb{Q})$; its inverse $q_{S}^{-1}$ defines an element of $\mathrm{H}^{2}(S, \mathbb{Q}) \otimes \mathrm{H}^{2}(S, \mathbb{Q})$. By the Künneth formula, we may view $q_{S}^{-1}$ as an element of $\mathrm{H}^{4}(S \times S, \mathbb{Q})$ that we denote $\mathfrak{B}$ and call the Beauville-Bogomolov class. The description above immediately gives that the cohomology class of $\pi_{S}^{2}$ in $\mathrm{H}^{4}(S \times S, \mathbb{Q})$ is $\mathfrak{B}$. Let us denote $\mathfrak{b}:=\iota_{\Delta}^{*} \mathfrak{B}$ and $\mathfrak{b}_{i}=p_{i}^{*} \mathfrak{b}$, where $p_{i}: S \times S \rightarrow S$ is the $i^{\text {th }}$ projection, $i=1,2$. On the one hand, a cohomological computation yields

$$
\mathfrak{B}=\left[\Delta_{S}\right]-\frac{1}{22}\left(\mathfrak{b}_{1}+\mathfrak{b}_{2}\right) .
$$

On the other hand, the cycle $L:=\Delta_{S}-\mathfrak{o}_{S} \times S-S \times \mathfrak{o}_{S}$, which was previously denoted $\pi_{S}^{2}$, satisfies $\iota_{\Delta}^{*} L=c_{2}(S)-2 \mathfrak{o}_{S}=22 \mathfrak{o}_{S}$. Thus not only does $L$ lift $\mathfrak{B}$ to rational equivalence, but $L$ also lifts the above equation satisfied by $\mathfrak{B}$ in the sense that

$$
L=\Delta_{S}-\frac{1}{22}\left(l_{1}+l_{2}\right)
$$

in the Chow group $\mathrm{CH}^{2}(S \times S)$, where $l:=\iota_{\Delta}^{*} L$ and $l_{i}:=p_{i}^{*} l, i=1,2$. Moreover, a cycle $L$ that satisfies the equation $L=\Delta_{S}-\frac{1}{22}\left(\left(p_{1}^{*} \iota_{\Delta}^{*} L\right)+\left(p_{2}^{*} \iota_{\Delta}^{*} L\right)\right)$ is unique. Indeed, if $L+\varepsilon$ is another cycle such that $L+\varepsilon=\Delta_{S}-\frac{1}{22}\left(p_{1}^{*} \iota_{\Delta}^{*}(L+\varepsilon)+p_{2}^{*} \iota_{\Delta}^{*}(L+\varepsilon)\right)$, then $\varepsilon=-\frac{1}{22}\left(p_{1}^{*} \iota_{\Delta}^{*} \varepsilon+p_{2}^{*} \iota_{\Delta}^{*} \varepsilon\right)$. Consequently, $\iota_{\Delta}^{*} \varepsilon=-\frac{1}{11} \iota_{\Delta}^{*} \varepsilon$. Thus $\iota_{\Delta}^{*} \varepsilon=0$ and hence $\varepsilon=0$.

We may then state a refinement of the main result of [11].

Theorem (Beauville-Voisin, revisited). Let $S$ be a complex projective K3 surface. Then there exists a unique 2-cycle $L \in \mathrm{CH}^{2}(S \times S)$ such that $L=\Delta_{S}-\frac{1}{22}\left(l_{1}+l_{2}\right)$. Moreover, $L$ represents the BeauvilleBogomolov class $\mathfrak{B}$ on $S$ and the Fourier transform associated to $L$ induces a splitting of the Chow ring $\mathrm{CH}^{*}(S)$. 
MINGMIN SHEN AND CHARLES VIAL

C. Hyperkähler varieties of $K 3^{[2]}$-type. Hyperkähler manifolds are simply connected compact Kähler manifolds $F$ such that $\mathrm{H}^{0}\left(F, \Omega_{F}^{2}\right)$ is spanned by a nowhere degenerate 2-form. This class of manifolds constitutes a natural generalization of the notion of a K3 surface in higher dimensions. Such manifolds are endowed with a canonical symmetric bilinear form $q_{F}: \mathrm{H}^{2}(F, \mathbb{Z}) \otimes \mathrm{H}^{2}(F, \mathbb{Z}) \rightarrow \mathbb{Z}$ called the BeauvilleBogomolov form. Passing to rational coefficients, its inverse $q_{F}^{-1}$ defines an element of $\mathrm{H}^{2}(F, \mathbb{Q}) \otimes \mathrm{H}^{2}(F, \mathbb{Q})$ and we define the Beauville-Bogomolov class $\mathfrak{B}$ in $\mathrm{H}^{4}(F \times F, \mathbb{Q})$ to be the class corresponding to $q_{F}^{-1}$ via the Künneth formula. In this manuscript, we will mostly be concerned with projective hyperkähler manifolds and these will simply be called hyperkähler varieties. A hyperkähler variety is said to be of $\mathrm{K} 3^{[n]}$-type if it is deformation equivalent to the Hilbert scheme of length- $n$ subschemes on a K3 surface. A first result is that if $F$ is a hyperkähler variety of $\mathrm{K} 3^{[n]}$-type, then there is a cycle $L \in \mathrm{CH}^{2}(F \times F)$ defined in (53) whose cohomology class is $\mathfrak{B} \in \mathrm{H}^{4}(F \times F, \mathbb{Q})$; see Theorem 9.15.

C.1. The Fourier decomposition for the Chow groups. Given a hyperkähler variety $F$ of K3 ${ }^{[2]}{ }_{-}$ type endowed with a cycle $L$ with cohomology class $\mathfrak{B}$, we define a descending filtration $\mathrm{F}^{\bullet}$ on the Chow groups $\mathrm{CH}^{i}(F)$ as

$$
\mathrm{F}^{2 k+1} \mathrm{CH}^{i}(F)=\mathrm{F}^{2 k+2} \mathrm{CH}^{i}(F):=\operatorname{ker}\left\{\left(L^{4-i+k}\right)_{*}: \mathrm{F}^{2 k} \mathrm{CH}^{i}(F) \rightarrow \mathrm{CH}^{4-i+2 k}(F)\right\} .
$$

The cohomological description of the powers of $[L]=\mathfrak{B}$ given in Proposition 1.3 shows that this filtration should be of Bloch-Beilinson type. We then define the Fourier transform as

$$
\mathcal{F}(\sigma)=\left(p_{2}\right)_{*}\left(e^{L} \cdot p_{1}^{*} \sigma\right), \quad \text { for all } \sigma \in \mathrm{CH}^{*}(F) .
$$

In this work, we ask if there is a canonical choice of a codimension-2 cycle $L$ on $F \times F$ lifting the Beauville-Bogomolov class $\mathfrak{B}$ such that the Fourier transform associated to $L$ induces a splitting of the conjectural Bloch-Beilinson filtration on the Chow group $\mathrm{CH}^{*}(F)$ compatible with its ring structure given by intersection product. We give positive answers in the case when $F$ is either the Hilbert scheme of length-2 subschemes on a K3 surface or the variety of lines on a cubic fourfold.

Consider now a projective hyperkähler manifold $F$ of $K 3^{[2]}$-type and let $\mathfrak{B}$ denote its BeauvilleBogomolov class, that is, the inverse of its Beauville-Bogomolov form seen as an element of $\mathrm{H}^{4}(F \times F, \mathbb{Q})$. We define $\mathfrak{b}:=\iota_{\Delta}^{*} \mathfrak{B}$ and $\mathfrak{b}_{i}:=p_{i}^{*} \mathfrak{b}$. Proposition 1.3 shows that $\mathfrak{B}$ is uniquely determined up to sign by the following quadratic equation :

$$
\mathfrak{B}^{2}=2\left[\Delta_{F}\right]-\frac{2}{25}\left(\mathfrak{b}_{1}+\mathfrak{b}_{2}\right) \cdot \mathfrak{B}-\frac{1}{23 \cdot 25}\left(2 \mathfrak{b}_{1}^{2}-23 \mathfrak{b}_{1} \mathfrak{b}_{2}+2 \mathfrak{b}_{2}^{2}\right) \quad \text { in } \mathrm{H}^{8}(F \times F, \mathbb{Q})
$$

We address the following ; see also Conjecture 2.1 for a more general version involving hyperkähler fourfolds whose cohomology ring is generated by degree-2 classes.

Conjecture 1. Let $F$ be a hyperkähler variety $F$ of $\mathrm{K} 3^{[2]}$-type. Then there exists a cycle $L \in \mathrm{CH}^{2}(F \times F)$ with cohomology class $\mathfrak{B} \in \mathrm{H}^{4}(F \times F, \mathbb{Q})$ satisfying

$$
L^{2}=2 \Delta_{F}-\frac{2}{25}\left(l_{1}+l_{2}\right) \cdot L-\frac{1}{23 \cdot 25}\left(2 l_{1}^{2}-23 l_{1} l_{2}+2 l_{2}^{2}\right) \quad \text { in } \mathrm{CH}^{4}(F \times F),
$$

where by definition we have set $l:=\iota_{\Delta}^{*} L$ and $l_{i}:=p_{i}^{*} l$.

In fact, we expect the symmetric cycle $L$ defined in (53) to satisfy the quadratic equation (6). Moreover, we expect a symmetric cycle $L \in \mathrm{CH}^{2}(F)$ representing the Beauville-Bogomolov class $\mathfrak{B}$ to be uniquely determined by the quadratic equation (6) ; see Proposition 3.4 for some evidence. From now on, when $F$ is the Hilbert scheme of length- 2 subschemes on a K3 surface, $F$ is endowed with the cycle $L$ defined in $(92)$ - it agrees with the one defined in (53) by Proposition 16.1. When $F$ is the variety of lines on a cubic fourfold, $F$ is endowed with the cycle $L$ defined in (107) - although we do not give a proof, this cycle (107) should agree with the one defined in (53).

Our first result, upon which our work is built, is the following theorem ; see Theorem 14.5 and Theorem 19.2 .

Theorem 1. Let $F$ be either the Hilbert scheme of length-2 subschemes on a K3 surface or the variety of lines on a cubic fourfold. Then Conjecture 1 holds for $F$. 
Let us introduce the cycle $l:=\iota_{\Delta}^{*} L \in \mathrm{CH}^{2}(F)$ - it turns out that $l=\frac{5}{6} c_{2}(F)$ when $F$ is either the Hilbert scheme of length-2 subschemes on a K3 surface or the variety of lines on a cubic fourfold ; see (93) and (108). The following hypotheses, together with (6), constitute the key relations towards establishing a Fourier decomposition for the Chow groups of $F$ :

$$
\begin{aligned}
L_{*} l^{2} & =0 ; & & \\
L_{*}\left(l \cdot L_{*} \sigma\right) & =25 L_{*} \sigma & & \text { for all } \sigma \in \mathrm{CH}^{4}(F) ; \\
\left(L^{2}\right)_{*}\left(l \cdot\left(L^{2}\right)_{*} \tau\right) & =0 & & \text { for all } \tau \in \mathrm{CH}^{2}(F) .
\end{aligned}
$$

Indeed, Theorem 2 below shows that in order to establish the existence of a Fourier decomposition on the Chow groups of a hyperkähler variety of $\mathrm{K} 3^{[2]}$-type, it suffices to show that the cycle $L$ defined in (53) (which is a characteristic class of Markman's twisted sheaf [35]) satisfies (6), (7), (8) and (9). Note that Properties (8) and (9) describe the intersection of $l$ with 2-cycles on $F$.

Theorem 2. Let $F$ be a hyperkähler variety of $K^{[2]}$-type. Assume that there exists a cycle $L \in \mathrm{CH}^{2}(F \times$ $F$ ) representing the Beauville-Bogomolov class $\mathfrak{B}$ satisfying (6), (7), (8) and (9). For instance, $F$ could be either the Hilbert scheme of length-2 subschemes on a K3 surface endowed with the cycle L of (92) or the variety of lines on a cubic fourfold endowed with the cycle $L$ of (107). Denote

$$
\mathrm{CH}^{i}(F)_{s}:=\left\{\sigma \in \mathrm{CH}^{i}(F): \mathcal{F}(\sigma) \in \mathrm{CH}^{4-i+s}(F)\right\} .
$$

Then the Chow groups of $F$ split canonically as

$$
\begin{aligned}
& \mathrm{CH}^{0}(F)=\mathrm{CH}^{0}(F)_{0} ; \\
& \mathrm{CH}^{1}(F)=\mathrm{CH}^{1}(F)_{0} ; \\
& \mathrm{CH}^{2}(F)=\mathrm{CH}^{2}(F)_{0} \oplus \mathrm{CH}^{2}(F)_{2} ; \\
& \mathrm{CH}^{3}(F)=\mathrm{CH}^{3}(F)_{0} \oplus \mathrm{CH}^{3}(F)_{2} ; \\
& \mathrm{CH}^{4}(F)=\mathrm{CH}^{4}(F)_{0} \oplus \mathrm{CH}^{4}(F)_{2} \oplus \mathrm{CH}^{4}(F)_{4} .
\end{aligned}
$$

Moreover, we have

$$
\mathrm{CH}^{i}(F)_{s}=\mathrm{Gr}_{\mathrm{F}}^{s} \bullet \mathrm{CH}^{i}(F) \text {, where } \mathrm{F}^{\bullet} \text { denotes the filtration (4); }
$$

and

$$
\sigma \text { belongs to } \mathrm{CH}^{i}(F)_{s} \text { if and only if } \mathcal{F}(\sigma) \text { belongs to } \mathrm{CH}^{4-i+s}(F)_{s} \text {. }
$$

We give further evidence that the splitting obtained in Theorem 2 is the splitting of a conjectural filtration $\mathrm{F}^{\bullet}$ on $\mathrm{CH}^{*}(F)$ of Bloch-Beilinson type by showing that it arises as the splitting of a filtration induced by a Chow-Künneth decomposition of the diagonal ; see Theorem 3.3. Note that our indexing convention is such that the graded piece of the conjectural Bloch-Beilinson filtration $\mathrm{CH}^{i}(F)_{s}=\mathrm{Gr}_{\mathrm{F}}^{s} \mathrm{CH}^{i}(F)$ should only depend on the cohomology group $\mathrm{H}^{2 i-s}(F, \mathbb{Q})$, or rather, on the Grothendieck motive $\mathfrak{h}_{\text {hom }}^{2 i-s}(F)$.

The proof that the Chow groups of a hyperkähler variety of $\mathrm{K} 3^{[2]}$-type satisfying hypotheses $(6),(7)$, (8) and (9) have a Fourier decomposition as described in the conclusion of Theorem 2 is contained in Theorems $2.2 \& 2.4$. That the Hilbert scheme of length-2 subschemes on a K3 surface endowed with the cycle $L$ of (92) satisfies hypotheses (6), (7), (8) and (9) is Theorem 14.5, Proposition 14.6 and Proposition 14.8. That the variety of lines on a cubic fourfold endowed with the cycle $L$ of (107) satisfies hypotheses (6), (7), (8) and (9) is Theorem 19.2, Proposition 19.4 and Proposition 19.6.

Theorem 2 is of course reminiscent of the case of abelian varieties where the Fourier decomposition on the Chow groups is induced by the exponential of the Poincaré bundle. Beauville's proof relies essentially on the interplay of the Poincaré line bundle and the multiplication-by- $n$ homomorphisms. Those homomorphisms are used in a crucial way to prove the compatibility of the Fourier decomposition with the intersection product. The difficulty in the case of hyperkähler varieties is that there are no obvious analogues to the multiplication-by- $n$ morphisms ; see however Remark 21.11. Still, when $F$ is the variety of lines on a cubic fourfold, Voisin [53] defined a rational self-map $\varphi: F \rightarrow F$ as follows. For a general point $[l] \in F$ representing a line $l$ on $X$, there is a unique plane $\Pi$ containing $l$ which is tangent to $X$ along $l$. Thus $\Pi \cdot X=2 l+l^{\prime}$, where $l^{\prime}$ is the residue line. Then one defines $\varphi([l])=\left[l^{\prime}\right]$. In $\S 21$, we study the graph 
MINGMIN SHEN AND CHARLES VIAL

of $\varphi$ in depth and completely determine its class both modulo rational equivalence and homological equivalence. It turns out that the action of $\varphi$ on the Chow groups of $F$ respects the Fourier decomposition; see $\S 21.6$. Thus in many respects the rational map $\varphi$ may be considered as an "endomorphism" of $F$. The interplay of $\varphi$ with $L$ is used to prove many features of the Fourier decomposition on the Chow groups of $F$.

C.2. The Fourier decomposition for the Chow ring. As in the case of abelian varieties or K3 surfaces, we are interested in the compatibility of the Fourier decomposition of Theorem 2 with the ring structure of $\mathrm{CH}^{*}(F)$. In the hyperkähler case, this was initiated by Beauville [9] who considered the sub-ring of $\mathrm{CH}^{*}(F)$ generated by divisors on the Hilbert scheme of length-2 subschemes on a K3 surface, and then generalized by Voisin [54] who considered the sub-ring of $\mathrm{CH}^{*}(F)$ generated by divisors and the Chern classes of the tangent bundle when $F$ is either the Hilbert scheme of length-2 subschemes on a K3 surface or the variety of lines on a cubic fourfold :

Theorem (Beauville [9], Voisin [54]). Let $F$ be either the Hilbert scheme of length-2 subschemes on a K3 surface, or the variety of lines on a cubic fourfold. Then any polynomial expression $P\left(D_{i}, c_{2}(F)\right)$, $D_{i} \in \mathrm{CH}^{1}(F)$, which is homologically trivial vanishes in the Chow ring $\mathrm{CH}^{*}(F)$.

This theorem implies the existence of a zero-cycle $\mathfrak{o}_{F} \in \mathrm{CH}_{0}(F)$ which is the class of a point such that

$$
\left\langle\mathfrak{o}_{F}\right\rangle=\left\langle c_{2}(F)^{2}\right\rangle=\left\langle c_{2}(F)\right\rangle \cdot \mathrm{CH}^{1}(F)^{\cdot 2}=\mathrm{CH}^{1}(F)^{\cdot 4} .
$$

This latter result can already be restated, in the context of our Fourier decomposition, as follows ; see Theorem 4.6.

We ask whether

$$
\mathrm{CH}^{4}(F)_{0}=\left\langle l^{2}\right\rangle=\langle l\rangle \cdot \mathrm{CH}^{1}(F)_{0}^{\cdot 2}=\mathrm{CH}^{1}(F)_{0}^{\cdot 4} .
$$

$$
\mathrm{CH}^{i}(F)_{s} \cdot \mathrm{CH}^{j}(F)_{r} \subseteq \mathrm{CH}^{i+j}(F)_{r+s}, \quad \text { for all }(i, s),(j, r) .
$$

The following theorem answers this question affirmatively when $F$ is the Hilbert scheme of length-2 subschemes on a K3 surface or the variety of lines on a very general cubic fourfold. Hilbert schemes of length-2 subschemes on K3 surfaces are dense in the moduli of hyperkähler varieties of $\mathrm{K} 3^{[2]}$-type, and the varieties of lines on cubic fourfolds form an algebraic component of maximal dimension. Therefore Theorem 3 gives strong evidence that a Fourier decomposition on the Chow ring of hyperkähler varieties of $\mathrm{K} 3^{[2]}$-type should exist.

Theorem 3. Let $F$ be either the Hilbert scheme of length-2 subschemes on a K3 surface or the variety of lines on a very general cubic fourfold. Then

$$
\mathrm{CH}^{i}(F)_{s} \cdot \mathrm{CH}^{j}(F)_{r} \subseteq \mathrm{CH}^{i+j}(F)_{r+s}, \quad \text { for all }(i, s),(j, r) .
$$

Moreover equality holds except when $\mathrm{CH}^{i+j}(F)_{r+s}=\mathrm{CH}^{3}(F)_{2}$ or $\mathrm{CH}^{2}(F)_{0}$.

We actually show Theorem 3 in a few more cases. Indeed, it is shown in Theorem 6.5 that the existence of a Fourier decomposition on the Chow groups or Chow ring of a hyperkähler variety of $\mathrm{K} 3^{[2]}$-type is a birational invariant, so that the conclusion of Theorem 3 also holds for any hyperkähler fourfold that is birational to the Hilbert scheme of length-2 subschemes on a K3 surface or to the variety of lines on a very general cubic fourfold.

The proof of Theorem 3 uses in an essential way a Theorem of Beauville-Voisin [11] on the vanishing of the "modified diagonal" of the K3 surface $S$ in the case when $F$ is the Hilbert scheme $S^{[2]}$, while it uses in an essential way the rational self-map $\varphi: F \rightarrow F$ constructed by Voisin [53] when $F$ is the variety of lines on a cubic fourfold. Theorem 3 is proved for divisors in Section 4, while it is proved in full generality for $S^{[2]}$ in Theorem 15.8 and for the variety of lines on a very general cubic fourfold in Section 22. In the $S^{[2]}$ case, we prove in fact a stronger result : the Fourier decomposition is induced by a multiplicative Chow-Künneth decomposition of the diagonal in the sense of Definition 8.1 ; see Theorem 15.8.

In addition to stating the multiplicativity property of the Fourier decomposition, Theorem 3 also states that $\mathrm{CH}^{2}(F)_{2} \cdot \mathrm{CH}^{2}(F)_{2}=\mathrm{CH}^{4}(F)_{4}$. This equality, which also holds for the variety of lines on a (not necessarily very general) cubic fourfold, reflects at the level of Chow groups, as predicted by the Bloch-Beilinson conjectures, the fact that the transcendental part of the Hodge structure $\mathrm{H}^{4}(F, \mathbb{Q})$ is a sub-quotient of $\operatorname{Sym}^{2} \mathrm{H}^{2}(F, \mathbb{Q})$. A proof in the case of $S^{[2]}$ can be found in Proposition 12.9 and a proof 
in the case of the variety of lines on a cubic fourfold can be found in Proposition 20.3. It is also expected from the Bloch-Beilinson conjectures that $\mathrm{CH}^{2}(F)_{0 \text {,hom }}=0$; see Theorem 3.3. In fact, for $F=S^{[2]}$, this would essentially follow from the validity of Bloch's conjecture for $S$. Although we cannot prove such a vanishing, a direct consequence of Theorem 3 is that

$$
\mathrm{CH}^{1}(F) \cdot \mathrm{CH}^{2}(F)_{0, \text { hom }}=0 \text { and } \mathrm{CH}^{2}(F)_{0} \cdot \mathrm{CH}^{2}(F)_{0, \text { hom }}=0 .
$$

Propositions $22.4 \& 22.5$ show that the above identities also hold when $F$ is the variety of lines on a (not necessarily very general) cubic fourfold.

Furthermore, we ought to mention that each piece of the decomposition of $\mathrm{CH}^{i}(F)$ obtained in Theorem 2 is non-trivial. Indeed, we have $\mathrm{CH}^{4}(F)_{0}=\left\langle l^{2}\right\rangle$ and $l \cdot: \mathrm{CH}^{2}(F)_{2} \rightarrow \mathrm{CH}^{4}(F)$ is injective with image $\mathrm{CH}^{4}(F)_{2}$; see Theorems $2.2 \&$ 2.4. Therefore, $\mathrm{CH}^{4}(F)_{0} \oplus \mathrm{CH}^{4}(F)_{2}$ is supported on a surface. Since $\mathrm{H}^{4}\left(F, \mathcal{O}_{F}\right) \neq 0$, it follows from Bloch-Srinivas [14] that $\mathrm{CH}^{4}(F)_{4} \neq 0$. Thus, because $\mathrm{CH}^{2}(F)_{2} \cdot \mathrm{CH}^{2}(F)_{2}=\mathrm{CH}^{4}(F)_{4}, \mathrm{CH}^{2}(F)_{2} \neq 0$ and hence $\mathrm{CH}^{4}(F)_{2} \neq 0$.

A direct consequence of Theorems $2 \& 3$ for zero-dimensional cycles is the following theorem which is analogous to the decomposition of the Chow group of zero-cycles on an abelian variety as can be found in [8, Proposition 4].

Theorem 4. Let $F$ be either the Hilbert scheme of length-2 subschemes on a K3 surface or the variety of lines on a cubic fourfold. Then

$$
\mathrm{CH}^{4}(F)=\left\langle l^{2}\right\rangle \oplus\langle l\rangle \cdot L_{*} \mathrm{CH}^{4}(F) \oplus\left(L_{*} \mathrm{CH}^{4}(F)\right)^{\cdot 2} .
$$

Moreover, this decomposition agrees with the Fourier decomposition of Theorem 2.

Let us point out that, with the notations of Theorem $2, L_{*} \mathrm{CH}^{4}(F)=\mathrm{CH}^{2}(F)_{2}$. A hyperkähler variety is simply connected. Thus its first Betti number vanishes and a theorem of Rojtman [45] implies that $\mathrm{CH}_{\mathbb{Z}}^{4}(F)_{\text {hom }}$ is uniquely divisible. Therefore, Theorem 4 can actually be stated for 0 -dimensional cycles with integral coefficients :

$$
\mathrm{CH}_{\mathbb{Z}}^{4}(F)=\mathbb{Z} \mathfrak{o}_{F} \oplus\langle l\rangle \cdot\left(L_{*} \mathrm{CH}^{4}(F)\right) \oplus\left(L_{*} \mathrm{CH}^{4}(F)\right)^{\cdot 2} .
$$

Finally, let us mention the following remark which could be useful to future work. As it is not essential to the work presented here, the details are not expounded. Given a divisor $D$ on $F$ with $q_{F}([D]) \neq 0$ where $q_{F}$ denotes the quadratic form attached to the Beauville-Bogomolov form, the cycle $L_{D}:=L-\frac{1}{q_{F}([D])} D_{1} \cdot D_{2}$, where $D_{i}:=p_{i}^{*} D, i=1,2$, defines a special Fourier transform $\mathcal{F}_{D}: \mathrm{CH}^{*}(F) \rightarrow$ $\mathrm{CH}^{*}(F), x \mapsto\left(p_{2}\right)_{*}\left(e^{L_{D}} \cdot p_{1}^{*} x\right)$ such that $\mathcal{F}_{D} \circ \mathcal{F}_{D}$ induces a further splitting of $\mathrm{CH}^{*}(F)$ which takes into account $D$. For instance, if $F$ is the variety of lines on a cubic fourfold and if $g$ is the Plücker polarization on $F$, then $\mathcal{F}_{g} \circ \mathcal{F}_{g}$ induces an orthogonal decomposition $\mathrm{CH}^{1}(F)=\langle g\rangle \oplus \mathrm{CH}^{1}(F)_{\text {prim }}$ but also a further decomposition $\mathrm{CH}^{3}(F)_{2}=A \oplus B$, where $\mathcal{F}_{g} \circ \mathcal{F}_{g}$ acts as the identity on $A$ and as zero on $B=g \cdot \mathrm{CH}^{2}(F)_{2}$. It turns out that $\varphi^{*}$ acts by multiplication by 4 on $A$ and by multiplication by -14 on $B$.

D. Hyperkähler varieties. Let $F$ be a hyperkähler variety. In general, the sub-algebra of $\mathrm{H}^{*}(F \times F, \mathbb{Q})$ generated by the Beauville-Bogomolov class $\mathfrak{B}, \mathfrak{b}_{1}$ and $\mathfrak{b}_{2}$ only "sees" the sub-Hodge structure of $\mathrm{H}^{*}(F, \mathbb{Q})$ generated by $\mathrm{H}^{2}(F, \mathbb{Q})$. Precisely, it can be checked that the cohomological Fourier transform $\mathcal{F}$ acts trivially on the orthogonal complement of the image of $\operatorname{Sym}^{n} \mathrm{H}^{2}(F, \mathbb{Q})$ inside $\mathrm{H}^{2 n}(F, \mathbb{Q})$. Therefore, it does not seem possible to formulate directly an analogous Fourier decomposition, as that of Theorems 2 $\& 3$, for the Chow ring of those hyperkähler varieties $F$ whose cohomology is not generated by $\mathrm{H}^{2}(F, \mathbb{Q})$. There are however three questions that we would like to raise concerning algebraic cycles on hyperkähler varieties. 
D.1. Zero-cycles on hyperkähler varieties. If $\omega$ is a nowhere degenerate 2-form on a hyperkähler variety $F$, then the powers $\omega^{n} \in \operatorname{Sym}^{n} \mathrm{H}^{2}(F, \mathbb{C})$ of $\omega$ span the degree-zero graded part of the cohomology of $F$ for the coniveau filtration. Hence we can expect that if a canonical 2-cycle $L \in \mathrm{CH}^{2}(F \times F)$ representing the Beauville-Bogomolov class $\mathfrak{B}$ exists, then the Fourier transform $\mathcal{F}:=e^{L}$ splits a BlochBeilinson type filtration on $\mathrm{CH}_{0}(F)$. It is thus tempting to ask whether the decomposition of Theorem 4 holds for hyperkähler varieties :

Conjecture 2. Let $F$ be a hyperkähler variety of dimension $2 d$. Then there exists a canonical cycle $L \in \mathrm{CH}^{2}(F \times F)$ representing the Beauville-Bogomolov class $\mathfrak{B}$ which induces a canonical splitting

$$
\mathrm{CH}^{2 d}(F)=\bigoplus_{s=0}^{d} \mathrm{CH}^{2 d}(F)_{2 s},
$$

where $\mathrm{CH}^{2 d}(F)_{2 s}:=\left\{\sigma \in \mathrm{CH}^{2 d}(F): \mathcal{F}(\sigma) \in \mathrm{CH}^{2 s}(F)\right\}$. Moreover, we have

$$
\mathrm{CH}^{2 d}(F)_{2 s}=\left\langle l^{d-s}\right\rangle \cdot\left(L_{*} \mathrm{CH}^{2 d}(F)\right)^{\cdot s}
$$

and

$$
\mathrm{CH}^{2 d}(F)_{2 s} \supseteq P\left(l, D_{1}, \ldots, D_{r}\right) \cdot\left(L_{*} \mathrm{CH}^{2 d}(F)\right)^{\cdot s},
$$

for any degree $2 d-2 s$ weighted homogeneous polynomial $P$ in $l$ and divisors $D_{i}, i=1, \ldots, r$.

Note that in the case of hyperkähler varieties of $\mathrm{K} 3^{[n]}$-type, a candidate for a canonical cycle $L$ representing $\mathfrak{B}$ is given by Theorem 9.15 . Note also that, in general, because $[L]=\mathfrak{B}$ induces an isomorphism $\mathrm{H}^{2 d-2}(F, \mathbb{Q}) \rightarrow \mathrm{H}^{2}(F, \mathbb{Q})$ a consequence of the Bloch-Beilinson conjectures would be that $L_{*} \mathrm{CH}^{2 d}(F)=\operatorname{ker}\left\{A J^{2}: \mathrm{CH}^{2}(F)_{\text {hom }} \rightarrow J^{2}(F) \otimes \mathbb{Q}\right\}$, where $A J^{2}$ denotes Griffiths' Abel-Jacobi map tensored with $\mathbb{Q}$.

D.2. On the existence of a distinguished cycle $L \in \mathrm{CH}^{2}(F \times F)$. Let $F$ be a hyperkähler variety. Beauville [9] conjectured that the sub-algebra $V_{F}$ of $\mathrm{CH}^{*}(F)$ generated by divisors injects into cohomology via the cycle class map. Voisin [54] conjectured that if one adds to $V_{F}$ the Chern classes of the tangent bundle of $F$, then the resulting sub-algebra still injects into cohomology. The following conjecture, which is in the same vein as the conjecture of Beauville-Voisin, is rather speculative but as Theorem 5 shows, it implies the existence of a Fourier decomposition on the Chow ring of hyperkähler fourfolds of $\mathrm{K} 3^{[2]}$-type. Before we can state it, we introduce some notations. Let $X$ be a smooth projective variety, and let the $\mathrm{Q}$-vector space $\bigoplus_{n} \mathrm{CH}^{*}\left(X^{n}\right)$ be equipped with the algebra structure given by intersection product on each summand. Denote $p_{X^{n} ; i_{1}, \ldots, i_{k}}: X^{n} \rightarrow X^{k}$ the projection on the $\left(i_{1}, \ldots, i_{k}\right)^{\text {th }}$ factor for $1 \leq i_{1}<\ldots<i_{k} \leq n$, and $\iota_{\Delta, X^{n}}: X \rightarrow X^{n}$ the diagonal embedding. Given cycles $\sigma_{1}, \ldots, \sigma_{r} \in \bigoplus_{n} \mathrm{CH}^{*}\left(X^{n}\right)$, we define $V\left(X ; \sigma_{1}, \ldots, \sigma_{r}\right)$ to be the smallest sub-algebra of $\bigoplus_{n} \mathrm{CH}^{*}\left(X^{n}\right)$ that contains $\sigma_{1}, \ldots, \sigma_{r}$ and that is stable under $\left(p_{X^{n} ; i_{1}, \ldots, i_{k}}\right)_{*},\left(p_{X^{n} ; i_{1}, \ldots, i_{k}}\right)^{*},\left(\iota_{\Delta, X^{n}}\right)_{*}$ and $\left(\iota_{\Delta, X^{n}}\right)^{*}$.

Conjecture 3 (Generalization of Conjecture 1). Let $F$ be a hyperkähler variety. Then there exists a canonical cycle $L \in \mathrm{CH}^{2}(F \times F)$ representing the Beauville-Bogomolov class $\mathfrak{B} \in \mathrm{H}^{4}(F \times F, \mathbb{Q})$ such that the restriction of the cycle class map $\bigoplus_{n} \mathrm{CH}^{*}\left(F^{n}\right) \rightarrow \bigoplus_{n} \mathrm{H}^{*}\left(F^{n}, \mathbb{Q}\right)$ to $V\left(F ; L, c_{0}(F), c_{2}(F), \ldots, c_{2 d}(F), D_{1}, \ldots, D_{r}\right)$, for any $D_{i} \in \mathrm{CH}^{1}(F)$, is injective.

Note that in higher dimensions $l$ and $c_{2}(F)$ are no longer proportional in $\mathrm{H}^{4}(F, \mathbb{Q})$. This is the reason why we consider $V\left(F ; L, c_{2}(F), \ldots, c_{2 d}(F), D_{1}, \ldots, D_{r}\right)$ rather than $V\left(F ; L, D_{1}, \ldots, D_{r}\right)$. Conjecture 3 is very strong : Voisin [54, Conjecture 1.6] had already stated it in the case of K3 surfaces (in that case $L$ need not be specified as it is simply given by $\Delta_{S}-\mathfrak{o}_{S} \times S-S \times \mathfrak{o}_{S}$ ) and noticed [57, p.92] that it implies the finite dimensionality in the sense of Kimura [33] and O'Sullivan [42] of the Chow motive of $S$. The following theorem reduces the Fourier decomposition problem for the Chow ring of hyperkähler varieties of $\mathrm{K} 3^{[2]}$-type to a weaker form of Conjecture 3 that only involves $L$.

Theorem 5 (Theorem 8.18). Let $F$ be a hyperkähler variety of $\mathrm{K}^{[2]}$-type. Assume that $F$ satisfies the following weaker version of Conjecture 3 : there exists a cycle $L \in \mathrm{CH}^{2}(F \times F)$ representing the BeauvilleBogomolov class $\mathfrak{B}$ satisfying equation (6), and the restriction of the cycle class map $\bigoplus_{n} \mathrm{CH}^{*}\left(F^{n}\right) \rightarrow$ $\bigoplus_{n} \mathrm{H}^{*}\left(F^{n}, \mathbb{Q}\right)$ to $V(F ; L)$ is injective. Then $\mathrm{CH}^{*}(F)$ admits a Fourier decomposition as in Theorem 2 which is compatible with its ring structure. 
This provides an approach to proving the Fourier decomposition for the Chow ring of hyperkähler varieties of $\mathrm{K}^{[2]}$-type that would avoid having to deal with non-generic cycles. Conjecture 3 can be considered as an analogue for hyperkähler varieties of O'Sullivan's theorem [43] which is concerned with abelian varieties. In the same way that Conjecture 3 implies the existence of a Fourier decomposition for the Chow ring of hyperkähler varieties of $\mathrm{K} 3^{[2]}$-type, we explain in Section 7 how Beauville's Fourier decomposition theorem for abelian varieties can be deduced directly from O'Sullivan's theorem.

Finally, as already pointed out, the diagonal $\left[\Delta_{F}\right]$ cannot be expressed as a polynomial in $\mathfrak{B}, \mathfrak{b}_{1}$ and $\mathfrak{b}_{2}$ when $F$ is a hyperkähler variety whose cohomology ring is not generated by degree-2 cohomology classes. Nevertheless, the orthogonal projector on the sub-Hodge structure generated by $\mathrm{H}^{2}(F, \mathbb{Q})$ can be expressed as a polynomial in $\mathfrak{B}, \mathfrak{b}_{1}$ and $\mathfrak{b}_{2}$. If one believes in Conjecture 3 , this projector should in fact lift to a projector, denoted $\Pi$, modulo rational equivalence. In that case, it seems reasonable to expect $\Pi_{*} \mathrm{CH}^{*}(F)$ to be a sub-ring of $\mathrm{CH}^{*}(F)$ and to expect the existence of a Fourier decomposition with kernel $L$ on the ring $\Pi_{*} \mathrm{CH}^{*}(F)$.

D.3. Multiplicative Chow-Künneth decompositions. We already mentioned that the Fourier decomposition for the Chow ring of the Hilbert scheme of length-2 subschemes on a K3 surface or the variety of lines on a very general cubic fourfold is in fact induced by a Chow-Künneth decomposition of the diagonal ( $c f$. $\S 3$ for a definition). A smooth projective variety $X$ of dimension $d$ is said to admit a weakly multiplicative Chow-Künneth decomposition if it can be endowed with a Chow-Künneth decomposition $\left\{\pi_{X}^{i}: 0 \leq i \leq 2 d\right\}$ that induces a decomposition of the Chow ring of $X$. That is, writing

$$
\mathrm{CH}_{\mathrm{CK}}^{i}(X)_{s}:=\left(\pi_{X}^{2 i-s}\right)_{*} \mathrm{CH}^{i}(X),
$$

we have

$$
\mathrm{CH}_{\mathrm{CK}}^{i}(X)_{s} \cdot \mathrm{CH}_{\mathrm{CK}}^{j}(X)_{r} \subseteq \mathrm{CH}_{\mathrm{CK}}^{i+j}(X)_{r+s}, \quad \text { for all }(i, s),(j, r) .
$$

The Chow-Künneth decomposition is said to be multiplicative if the above holds at the level of correspondences ; see Definition 8.1.

Together with Murre's conjecture (D) as stated in $\S 3$, we ask :

Conjecture 4. Let $X$ be a hyperkähler variety. Then $X$ can be endowed with a Chow-Künneth decomposition that is multiplicative. Moreover, the cycle class map $\mathrm{CH}^{i}(X) \rightarrow \mathrm{H}^{2 i}(X, \mathbb{Q})$ restricted to $\mathrm{CH}_{\mathrm{CK}}^{i}(X)_{0}$ is injective.

Note that if $A$ is an abelian variety, then $A$ has a multiplicative Chow-Künneth decomposition ( $c f$. Example 8.3) ; and that the cycle class map restricted to the degree-zero graded part of the Chow ring for the Chow-Künneth decomposition is injective was asked by Beauville [8]. Note also that Conjecture 3 and Conjecture 4 have non-trivial intersection. Indeed, if $X$ is a hyperkähler variety, then provided that $\mathfrak{B}$ is algebraic (which is the case if $X$ is of $\mathrm{K} 3^{[n]}$-type by Theorem 9.15), then by invoking Lemma 8.7 we see that Conjecture 3 for $X$ is implied by Conjecture 4 for $X$ and by knowing that the Chern classes $c_{i}(X) \in \mathrm{CH}^{i}(X)$ of $X$ belong to $\mathrm{CH}_{\mathrm{CK}}^{i}(X)_{0}$. As a partial converse, Theorem 8.18 shows that a hyperkähler variety of $\mathrm{K} 3^{[2]}$-type for which Conjecture 3 holds admits a multiplicative Chow-Künneth decomposition.

In Section 8, we define the notion of multiplicative Chow-Künneth decomposition and discuss its relevance as well as its links with so-called modified diagonals (cf. Proposition 8.12). In Section 13, we prove that the Hilbert scheme of length-2 subschemes on a K3 surface not only admits a weakly multiplicative Chow-Künneth decomposition but admits a multiplicative Chow-Künneth decomposition (as predicted by Conjecture 3). More generally, we produce many examples of varieties that can be endowed with a multiplicative Chow-Künneth decomposition. In $\S 13.4$, we prove :

Theorem 6. Let $E$ be the smallest sub-set of smooth projective varieties that contains varieties with Chow groups of finite rank (as $\mathbb{Q}$-vector spaces), abelian varieties, symmetric products of hyperelliptic curves, and $K 3$ surfaces, and that is stable under the following operations :

(i) if $X$ and $Y$ belong to $E$, then $X \times Y \in E$;

(ii) if $X$ belongs to $E$, then $\mathbb{P}\left(\mathscr{T}_{X}\right) \in E$, where $\mathscr{T}_{X}$ is the tangent bundle of $X$; 
(iii) if $X$ belongs to $E$, then the blow-up of $X \times X$ along the diagonal belongs to $E$;

(iv) if $X$ belongs to $E$, then the Hilbert scheme of length-2 subschemes $X^{[2]} \in E$.

Let $X$ be a smooth projective variety that is isomorphic to a variety in $E$. Then $X$ admits a multiplicative Chow-Künneth decomposition.

It is intriguing to notice that all the examples that we produce satisfy $c_{i}(X) \in \mathrm{CH}_{\mathrm{CK}}^{i}(X)_{0}$.

Abelian varieties and hyperkähler varieties are instances of varieties with vanishing first Chern class. In fact a classical theorem of Beauville [6] and Bogomolov [15] states that every smooth projective variety $X$ with vanishing first Chern class is, up to finite étale cover, isomorphic to the product of an abelian variety with hyperkähler varieties and Calabi-Yau varieties. However, one cannot expect varieties with vanishing first Chern class to have a multiplicative (and even a weakly multiplicative) Chow-Künneth decomposition : Beauville constructed Calabi-Yau threefolds $X$ [9, Example 2.1.5(b)] that do not satisfy the weak splitting property. Precisely, Beauville's examples have the property that there exist two divisors $D_{1}$ and $D_{2}$ such that $D_{1} \cdot D_{2} \neq 0 \in \mathrm{CH}^{2}(X)$ but such that $\left[D_{1}\right] \cup\left[D_{2}\right]=0 \in \mathrm{H}^{4}(X, \mathbb{Q})$. To conclude, we ask : what is a reasonable class of varieties, containing abelian varieties and hyperkähler varieties, that we can expect to have a multiplicative Chow-Künneth decomposition? 
Outline. The manuscript is divided into three parts. We have tried to extract the key properties that a cycle $L$ representing the Beauville-Bogomolov class $\mathfrak{B}$ must satisfy in order to induce a Fourier decomposition on the Chow groups of a hyperkähler fourfold of $\mathrm{K} 3^{[2]}$-type. As a consequence the sections are not organized in a linear order.

We first introduce in $\S 1$ the Beauville-Bogomolov class $\mathfrak{B}$ and establish in Proposition 1.3 the quadratic equation (5). The core of Part 1 then consists in Theorems $2.2 \& 2.4$ and Theorem 3.3, where we consider a hyperkähler variety $F$ of $\mathrm{K}^{[2]}$-type endowed with a cycle $L \in \mathrm{CH}^{2}(F \times F)$ representing the BeauvilleBogomolov class $\mathfrak{B}$ that satisfies (6), (7), (8) and (9), and show that the conclusion of Theorem 2 holds for $F$ and that the resulting Fourier decomposition on the Chow groups of $F$ is in fact induced by a Chow-Künneth decomposition. We then assume that we have proved the existence of a cycle $L$ representing the Beauville-Bogomolov class satisfying hypotheses (6), (7), (8) and (9) when $F$ is either the Hilbert scheme of length-2 subschemes on a K3 surface or the variety of lines on a cubic fourfold. We then consider in $\S 4$ the sub-algebra $V_{F}$ of $\mathrm{CH}^{*}(F)$ generated by divisors and $l$. With the formalism of the Fourier decomposition in mind, we show that the results of Beauville [9] and Voisin [54] which state that $V_{F}$ injects into cohomology via the cycle class map can be reinterpreted as saying that $V_{F}$ lies in the degree-zero graded part of our filtration. Proposition 5.1 explains how Conjecture 2 is helpful to understanding the action of automorphisms on zero-cycles of hyperkähler varieties. An application to symplectic automorphisms of $S^{[2]}$ is given. We show in $\S 6$ that the multiplicativity property of the Fourier decomposition boils down to intersection-theoretic properties of the cycle $L$. We deduce in Theorem 6.5 that the Fourier decomposition is a birational invariant for hyperkähler varieties of $\mathrm{K}^{[2]}$-type. This approach is used in $\S 7$ to give new insight on the theory of algebraic cycles on abelian varieties by showing how Beauville's Fourier decomposition theorem [8] is a direct consequence of a recent theorem of O'Sullivan [43]. Section 8 introduces the notion of multiplicative Chow-Künneth decomposition and its relevance is discussed. In particular, we relate this notion to the notion of so-called modified diagonals, give first examples of varieties admitting a multiplicative Chow-Künneth decomposition, and prove a more precise version of Theorem 5. Part 1 ends with $\S 9$ where a proof of the algebraicity of the BeauvilleBogomolov class $\mathfrak{B}$ is given for hyperkähler varieties of $\mathrm{K} 3^{[n]}$-type.

Part 2 and Part 3 are devoted to proving Theorem 1, Theorem 2 and Theorem 3 for the Hilbert scheme of length-2 subschemes on a K3 surface and for the variety of lines on a cubic fourfold, respectively. In both cases, the strategy for proving Theorem 1 and Theorem 2 consists in first studying the incidence correspondence $I$ and its intersection-theoretic properties, and then in constructing a cycle $L \in \mathrm{CH}^{2}(F \times$ $F$ ) very close to $I$ representing the Beauville-Bogomolov class satisfying hypotheses (6), (7), (8) and (9).

Actually, Part 2 begins by considering the incidence correspondence $I$ for the Hilbert scheme $X^{[2]}$ for any smooth projective variety $X$. In that generality, we establish in $\S 11$ some equations satisfied by $I$ and deduce in $\S 12$ some splitting results for the action of $I^{2}=I \cdot I$ on $\mathrm{CH}_{0}\left(X^{[2]}\right)$. We then gradually add some constraints on $X$ which are related to degeneration properties of the modified diagonal. In $\S 13$, we study in depth the notion of multiplicative Chow-Künneth decomposition for $X$ and give sufficient conditions for it to be stable under blow-up and under taking the Hilbert scheme of length-2 points $X^{[2]}$. The main contribution there is the proof of Theorem 6 given in $\S 13.4$, and a notable intermediate result is Theorem 13.4 which shows in particular that $X^{[2]}$ admits a multiplicative Chow-Künneth decomposition when $X$ is a K3 surface or an abelian variety. In $\S 14$, we turn our focus exclusively on $S^{[2]}$ for a K3 surface $S$, and prove Theorem 1 (note the use of the crucial Lemma 14.3) and Theorem 2 in that case. In $\S 15$, we prove Theorem 3 by showing that the Fourier decomposition of the Chow group $\mathrm{CH}^{*}\left(S^{[2]}\right)$ agrees with the multiplicative Chow-Künneth decomposition of Theorem 13.4 obtained by considering the multiplicative Chow-Künneth decomposition for $S$ of Example 8.17. Finally, in $\S 16$, we prove that the cycle $L$ constructed in $\$ 14.2$ using the incidence correspondence coincides with the cycle $L$ constructed in $§ 9$ using Markman's twisted sheaf.

The structure of Part 3 is more straightforward. We define the incidence correspondence $I$ and Voisin's rational self-map $\varphi$ for $F$, the variety of lines on a cubic fourfold, and study their interaction when acting on the Chow groups of $F$. This leads in $\S 19$ to a proof of Theorems $1 \& 2$ in that case. The goal of $\S 20$ is to prove that $\mathrm{CH}^{4}(F)_{4}=\mathrm{CH}^{2}(F)_{2} \cdot \mathrm{CH}^{2}(F)_{2}$. While such a statement was only a matter of combinatorics in the case of $S^{[2]}$, here we have to resort to an analysis of the geometry of cubic fourfolds ; see Theorem 
20.5 in this respect. Finally, in order to complete the proof of Theorem 3 in $\S 22$, we use the rational map $\varphi$ in an essential way. For that matter, the main result of $\S 21$ is Proposition 21.2, a nice consequence of which is Theorem 21.9 which shows that the action of $\varphi^{*}$ on $\mathrm{CH}^{*}(F)$ is diagonalizable when $X$ does not contain any plane. For the sake of completeness and clarity, we have gathered general results used throughout Part 3 about cubic fourfolds and about the action of rational maps on Chow groups in two separate and self-contained appendices.

Conventions. We work over the field of complex numbers. Chow groups $\mathrm{CH}^{i}$ are with rational coefficients and we use $\mathrm{CH}_{\mathbb{Z}}^{i}$ to denote the Chow groups with integral coefficients. If $X$ is a variety, $\mathrm{CH}^{i}(X)_{\text {hom }}$ is the kernel of the cycle class map that sends a cycle $\sigma \in \mathrm{CH}^{i}(X)$ to its cohomology class $[\sigma] \in \mathrm{H}^{2 i}(X, \mathbb{Q})$. By definition we set $\mathrm{CH}^{j}(X)=0$ for $j<0$ and for $j>\operatorname{dim} X$. If $Y$ is another variety and if $\gamma$ is a correspondence in $\mathrm{CH}^{i}(X \times Y)$, its transpose ${ }^{t} \gamma \in \mathrm{CH}^{i}(Y \times X)$ is the image of $\gamma$ under the action of the permutation map $X \times Y \rightarrow Y \times X$. If $\gamma_{1}, \cdots, \gamma_{n}$ are correspondences in $\mathrm{CH}^{*}(X \times Y)$, then the correspondence $\gamma_{1} \otimes \cdots \otimes \gamma_{n} \in \mathrm{CH}^{*}\left(X^{n} \times Y^{n}\right)$ is defined as $\gamma_{1} \otimes \cdots \otimes \gamma_{n}=\prod_{i=1}^{n}\left(p_{i, n+i}\right)^{*} \gamma_{i}$, where $p_{i, n+i}: X^{n} \times Y^{n} \rightarrow X \times Y$ is the projection on the $i^{\text {th }}$ and $(n+i)^{\text {th }}$ factors.

Acknowledgments. We would like to thank Daniel Huybrechts for his interest and for explaining [35]. We are also very grateful to two referees for their detailed comments that have helped improve the exposition. 


\section{Part 1. The Fourier transform for hyperkähler fourfolds}

\section{The COHOMOlOgical Fourier transform}

The goal of this section is to establish equation (5) satisfied by the Beauville-Bogomolov class $\mathfrak{B}$ of a hyperkähler manifold of $\mathrm{K}^{[2]}$-type, and to show that $\mathfrak{B}$ is uniquely determined, up to sign, by this equation. This is embodied in Proposition 1.3. Along the way, we express the Künneth projectors in terms of $\mathfrak{B}$; see Corollary 1.7 .

We start by defining the Beauville-Bogomolov class $\mathfrak{B}$ seen as an element of $\mathrm{H}^{4}(F \times F, \mathbb{Q})$. In a broader context, let us first consider a free abelian group $\Lambda$ and the associated vector space $V=\Lambda \otimes \mathbb{Q}$ (or $\Lambda \otimes \mathbb{C}$ ). A quadratic form (or equivalently a symmetric bilinear form) $q$ on $V$ can be viewed as an element of $\operatorname{Sym}^{2}\left(V^{*}\right)$. If $q$ is non-degenerate, then it induces an isomorphism $V \cong V^{*}$ which further gives an identification

$$
\operatorname{Sym}^{2}(V) \cong \operatorname{Sym}^{2}\left(V^{*}\right) .
$$

Under this identification, the element $q \in \operatorname{Sym}^{2}\left(V^{*}\right)$ gives rise to an element, denoted $q^{-1}$, of $\operatorname{Sym}^{2}(V)$. If we choose a basis $\left\{v_{1}, \ldots, v_{r}\right\}$ of $V$ and write $A:=\left(q\left(v_{i}, v_{j}\right)\right)_{1 \leq i, j \leq r}$, then

$$
q^{-1}=\sum_{1 \leq i, j \leq r} q_{i j} v_{i} \otimes v_{j} \in \operatorname{Sym}^{2} V
$$

where $\left(q_{i j}\right)_{1 \leq i, j \leq r}:=A^{-1}$.

We will be especially interested in the second cohomology group of a compact hyperkähler manifold, which carries a canonical bilinear form by the following theorem of Bogomolov, Beauville and Fujiki.

Theorem 1.1 (Bogomolov, Beauville, and Fujiki ; see [6, 24]). Let F be a compact hyperkähler manifold of dimension $2 n$. Then $\Lambda=\mathrm{H}^{2}(F, \mathbb{Z})$ is endowed with a canonical bilinear form $q_{F}$ which satisfies the Fujiki relation

$$
\int_{F} \alpha^{2 n}=\frac{(2 n) !}{2^{n} n !} c_{F} q_{F}(\alpha, \alpha)^{n}, \quad \forall \alpha \in \mathrm{H}^{2}(F, \mathbb{Z}) .
$$

The above bilinear form of Theorem 1.1 is called the Beauville-Bogomolov form and the constant $\frac{(2 n) !}{2^{n} n !} c_{F}$ is called the Fujiki constant. In this section, we deal with cup-product only for cohomology classes of even degree. Hence the symbol " $\cup$ " will frequently be omitted and $\alpha \beta$ will stand for $\alpha \cup \beta$.

Definition 1.2. Let $q_{F}$ be the Beauville-Bogomolov bilinear form on $\mathrm{H}^{2}(F, \mathbb{Z})$. Its inverse $q_{F}^{-1}$ defines an element of $\mathrm{H}^{2}(F, \mathbb{Z}) \otimes \mathrm{H}^{2}(F, \mathbb{Z}) \otimes \mathbb{Q}$, and the Beauville-Bogomolov class

$$
\mathfrak{B} \in \mathrm{H}^{4}(F \times F, \mathbb{Q})
$$

is defined to be the image of $0 \oplus q_{F}^{-1} \oplus 0 \in \mathrm{H}^{0}(F, \mathbb{Q}) \otimes \mathrm{H}^{4}(F, \mathbb{Q}) \oplus \mathrm{H}^{2}(F, \mathbb{Q}) \otimes \mathrm{H}^{2}(F, \mathbb{Q}) \oplus \mathrm{H}^{4}(F, \mathbb{Q}) \otimes \mathrm{H}^{0}(F, \mathbb{Q})$ under the Künneth decomposition.

The cohomological Fourier transform is the homomorphism

$$
[\mathcal{F}]: \mathrm{H}^{*}(F, \mathbb{Q}) \rightarrow \mathrm{H}^{*}(F, \mathbb{Q}), \quad x \mapsto p_{2 *}\left(e^{\mathfrak{B}} \cup p_{1}^{*} x\right),
$$

where $p_{i}: F \times F \rightarrow F$ is the projection onto the $i^{\text {th }}$ factor.

We write $\iota_{\Delta}: F \hookrightarrow F \times F$ for the diagonal embedding and we define

$$
\mathfrak{b}:=\iota_{\Delta}^{*} \mathfrak{B} \in \mathrm{H}^{4}(F, \mathbb{Q}) \quad \text { and } \quad \mathfrak{b}_{i}:=p_{i}^{*} \mathfrak{b} \in \mathrm{H}^{4}(F \times F, \mathbb{Q}) .
$$

From now on, we assume that $\operatorname{dim} F=4$. In this case, the Fujiki relation of Theorem 1.1 implies

$$
\int_{F} \alpha_{1} \alpha_{2} \alpha_{3} \alpha_{4}=c_{F}\left\{q_{F}\left(\alpha_{1}, \alpha_{2}\right) q_{F}\left(\alpha_{3}, \alpha_{4}\right)+q_{F}\left(\alpha_{1}, \alpha_{3}\right) q_{F}\left(\alpha_{2}, \alpha_{4}\right)+q_{F}\left(\alpha_{1}, \alpha_{4}\right) q_{F}\left(\alpha_{2}, \alpha_{3}\right)\right\}
$$

for all $\alpha_{1}, \alpha_{2}, \alpha_{3}, \alpha_{4} \in \Lambda=\mathrm{H}^{2}(F, \mathbb{Z})$. The following proposition describes the action of the powers of $\mathfrak{B} \in \mathrm{H}^{4}(F \times F, \mathbb{Q})$ on $\mathrm{H}^{*}(F, \mathbb{Q})$, when the cohomology ring of $F$ is generated by degree-2 classes. Note that by a result of Verbitsky, the cup-product map $\operatorname{Sym}^{2}\left(\mathrm{H}^{2}(F, \mathbb{Q})\right) \rightarrow \mathrm{H}^{4}(F, \mathbb{Q})$ is always injective ; see $[16]$. 
Proposition 1.3. Assume $F$ is a compact hyperkähler manifold of dimension 4 which satisfies

$$
\mathrm{H}^{4}(F, \mathbb{Q})=\operatorname{Sym}^{2}\left(\mathrm{H}^{2}(F, \mathbb{Q})\right) \quad \text { and } \mathrm{H}^{3}(F, \mathbb{Q})=0 .
$$

Let $r=\operatorname{dim} \mathrm{H}^{2}(F, \mathbb{Q})$ and let $c_{F}$ be a third of the Fujiki constant of $F$. Then the following are true.

(i) The following equality holds in $\mathrm{H}^{8}(F \times F, \mathbb{Q})$,

$$
\mathfrak{B}^{2}=2 c_{F}\left[\Delta_{F}\right]-\frac{2}{r+2}\left(\mathfrak{b}_{1}+\mathfrak{b}_{2}\right) \mathfrak{B}-\frac{1}{r(r+2)}\left(2 \mathfrak{b}_{1}^{2}-r \mathfrak{b}_{1} \mathfrak{b}_{2}+2 \mathfrak{b}_{2}^{2}\right) .
$$

(ii) The action of $\left(\mathfrak{B}^{k}\right)_{*}, k=0,1,2,3,4$, on $\mathrm{H}^{i}(F, \mathbb{Q})$ is zero for $i \neq 8-2 k$.

(iii) The action of $\left(\mathfrak{B}^{2}\right)_{*}$ on $\mathrm{H}^{4}(F, \mathbb{Q})$ gives an eigenspace decomposition

$$
\mathrm{H}^{4}(F, \mathbb{Q})=\langle\mathfrak{b}\rangle \oplus\langle\mathfrak{b}\rangle^{\perp}
$$

which is orthogonal for the intersection form and where $\left(\mathfrak{B}^{2}\right)_{*}=(r+2) c_{F}$ on $\langle\mathfrak{b}\rangle$ and $\left(\mathfrak{B}^{2}\right)_{*}=2 c_{F}$ on $\langle\mathfrak{b}\rangle^{\perp}$.

(iv) $\left(\mathfrak{B}^{k}\right)_{*} \circ\left(\mathfrak{B}^{4-k}\right)_{*}: \mathrm{H}^{2 k}(F, \mathbb{Q}) \rightarrow \mathrm{H}^{8-2 k}(F, \mathbb{Q}) \rightarrow \mathrm{H}^{2 k}(F, \mathbb{Q})$ is multiplication by $3(r+2) c_{F}^{2}$ when $k=1,3$ and is multiplication by $3 r(r+2) c_{F}^{2}$ when $k=0,4$.

(v) $\pm \mathfrak{B}$ is the unique element in $\mathrm{H}^{2}(F) \otimes \mathrm{H}^{2}(F)$ that satisfies the above equation (12) in the following sense. Assume that $\mathfrak{C} \in \mathrm{H}^{2}(F) \otimes \mathrm{H}^{2}(F)$ satisfies equation (12) with $\mathfrak{B}$ replaced by $\mathfrak{C}$ and $\mathfrak{b}_{i}$ replaced by $\mathfrak{c}_{i}$, where $\mathfrak{c}_{i}=p_{i}^{*} \mathfrak{c}$ and $\mathfrak{c}=\iota_{\Delta}^{*} \mathfrak{C}$. Then $\mathfrak{C}= \pm \mathfrak{B}$.

Before proving Proposition 1.3, we establish some auxiliary lemmas : Lemma 1.4 and Lemma 1.5 hold generally for any compact hyperkähler manifold of dimension 4, and Lemma 1.6 computes the class of the diagonal $\left[\Delta_{F}\right]$ in terms of the Beauville-Bogomolov class under the condition that the cohomology of $F$ is generated by degree-2 elements.

Let $V=\mathrm{H}^{2}(F, \mathbb{C})$, then the Beauville-Bogomolov bilinear form extends to $V \times V$. We choose an orthonormal basis $\left\{e_{1}, e_{2}, \ldots, e_{r}\right\}$ for $V$, namely $q_{F}\left(e_{i}, e_{j}\right)=\delta_{i j}$. Then we have

$$
\mathfrak{B}=e_{1} \otimes e_{1}+e_{2} \otimes e_{2}+\cdots+e_{r} \otimes e_{r}
$$

and $\mathfrak{b}=e_{1}^{2}+e_{2}^{2}+\cdots+e_{r}^{2}$

By Poincaré duality, we have

$$
\mathrm{H}^{6}(F, \mathbb{C})=\mathrm{H}^{2}(F, \mathbb{C})^{\vee} .
$$

This allows us to define the dual basis $\left\{e_{1}^{\vee}, e_{2}^{\vee}, \ldots, e_{r}^{\vee}\right\}$ of $\mathrm{H}^{6}(F, \mathbb{C})$.

With the above notions, we first note that if $1 \leq i, j, k \leq r$ are distinct then

$$
e_{i} e_{j} e_{k}=0, \quad \text { in } \mathrm{H}^{6}(F, \mathbb{C}) .
$$

This is because, by equation (11), one easily shows that $e_{i} e_{j} e_{k} e_{l}=0$ for all $1 \leq l \leq r$. We also note that

$$
\begin{gathered}
e_{i}^{2} e_{j}=c_{F} e_{j}^{\vee}, \quad 1 \leq i \neq j \leq r, \\
e_{j}^{3}=3 c_{F} e_{j}^{\vee}, \quad 1 \leq j \leq r .
\end{gathered}
$$

The equation (15) follows from the following computation

$$
e_{i}^{2} e_{j} e_{k}=c_{F}\left(q_{F}\left(e_{i}, e_{i}\right) q_{F}\left(e_{j}, e_{k}\right)+2 q_{F}\left(e_{i}, e_{j}\right) q_{F}\left(e_{i}, e_{k}\right)\right)=c_{F} \delta_{j k}, \quad i \neq j .
$$

Similarly, equation (16) follows from $e_{j}^{3} e_{k}=3 c_{F} q_{F}\left(e_{j}, e_{j}\right) q_{F}\left(e_{j}, e_{k}\right)=3 c_{F} \delta_{j k}$. Combining equations (15) and (16), we have

$$
\mathfrak{b} e_{i}=(r+2) c_{F} e_{i}^{\vee}, \quad 1 \leq i \leq r .
$$

Lemma 1.4. The following cohomological relations hold.

(i) $\mathfrak{B}^{2}=\sum_{i=1}^{r} e_{i}^{2} \otimes e_{i}^{2}+2 \sum_{1 \leq i<j \leq r} e_{i} e_{j} \otimes e_{i} e_{j}$.

(ii) $\mathfrak{B}^{3}=3(r+2) c_{F}^{2} \sum_{i=1}^{r} e_{i}^{\vee} \otimes e_{i}^{\vee}$.

(iii) $\mathfrak{B}^{4}=3 r(r+2) c_{F}^{2}[p t] \otimes[p t]$. 
Proof. Statement (i) follows by squaring both sides of (13). Statement (ii) can be derived as follows. First we use (i) and get

$$
\mathfrak{B}^{2}\left(e_{k} \otimes e_{k}\right)=\sum_{i=1^{r}} e_{i}^{2} e_{k} \otimes e_{i}^{2} e_{k}+2 \sum_{1 \leq i<j \leq r} e_{i} e_{j} e_{k} \otimes e_{i} e_{j} e_{k} .
$$

We invoke equations (14), (15) and (16) to find

$$
\mathfrak{B}^{2}\left(e_{k} \otimes e_{k}\right)=(r+6) c_{F}^{2} e_{k}^{\vee} \otimes e_{k}^{\vee}+2 c_{F}^{2} \sum_{i=1}^{r} e_{i}^{\vee} \otimes e_{i}^{\vee} .
$$

By taking the sum as $k$ runs through 1 to $r$, we get (ii). Statement (iii) can be proved similarly.

Lemma 1.5. The following cohomological relations hold.

(i) $\mathfrak{b}_{1} \mathfrak{B}=(r+2) c_{F} \sum_{i=1}^{r} e_{i}^{\vee} \otimes e_{i} \in \mathrm{H}^{6}(F) \otimes \mathrm{H}^{2}(F)$.

(ii) $\mathfrak{b}_{2} \mathfrak{B}=(r+2) c_{F} \sum_{i=1}^{r} e_{i} \otimes e_{i}^{\vee} \in \mathrm{H}^{2}(F) \otimes \mathrm{H}^{6}(F)$.

(iii) $\left(\mathfrak{b}_{1}\right)^{2}=r(r+2) c_{F}[p t] \otimes[F] \in \mathrm{H}^{8}(F) \otimes \mathrm{H}^{0}(F)$.

(iv) $\left(\mathfrak{b}_{2}\right)^{2}=r(r+2) c_{F}[F] \otimes[p t] \in \mathrm{H}^{0}(F) \otimes \mathrm{H}^{8}(F)$.

(v) $\mathfrak{b}_{1} \mathfrak{b}_{2}=\sum_{i, j=1}^{r} e_{i}^{2} \otimes e_{j}^{2} \in \mathrm{H}^{4}(F) \otimes \mathrm{H}^{4}(F)$.

Proof. Under the Künneth isomorphism, we have $\mathfrak{b}_{1}=\sum_{i=1}^{r} e_{i}^{2} \otimes 1$ and $\mathfrak{b}_{2}=\sum_{i=1}^{r} 1 \otimes e_{i}^{2}$. Hence we get

$$
\mathfrak{b}_{1} \mathfrak{B}=\sum_{i, j=1}^{r} e_{i}^{2} e_{j} \otimes e_{j}=\sum_{j=1}^{r}\left(\mathfrak{b} e_{j}\right) \otimes e_{j}=(r+2) c_{F} \sum_{j=1}^{r} e_{j}^{\vee} \otimes e_{j},
$$

which proves (i). Statement (ii) can be proved similarly. Statements (iii) and (iv) follow from $\mathfrak{b}^{2}=$ $r(r+2) c_{F}[p t]$. Statement $(v)$ follows from the above explicit expressions for $\mathfrak{b}_{1}$ and $\mathfrak{b}_{2}$.

Lemma 1.6. Under the assumption of Proposition 1.3, the cohomology class of the diagonal $\Delta_{F}$ is given by

$$
\begin{aligned}
{\left[\Delta_{F}\right]=} & \sum_{i=1}^{r}\left(e_{i}^{\vee} \otimes e_{i}+e_{i} \otimes e_{i}^{\vee}\right)+\frac{1}{2 c_{F}} \sum_{i=1}^{r} e_{i}^{2} \otimes e_{i}^{2}+\frac{1}{c_{F}} \sum_{1 \leq i<j \leq r} e_{i} e_{j} \otimes e_{i} e_{j} \\
& -\frac{1}{2(r+2) c_{F}} \sum_{i, j=1}^{r} e_{i}^{2} \otimes e_{j}^{2}+[p t] \otimes[F]+[F] \otimes[p t] .
\end{aligned}
$$

Proof. We need to check that the right-hand side of (18) acts as the identity on cohomology. Here we only check this on $\mathrm{H}^{4}(F, \mathbb{Q})$. The other cases are easy to check. Note that if $1 \leq i<j \leq r$ then

$$
\left(e_{i} e_{j} \otimes e_{i} e_{j}\right)_{*}\left(e_{k} e_{l}\right)=\left(e_{i} e_{j} e_{k} e_{l}\right) e_{i} e_{j}=c_{F}\left(\delta_{i k} \delta_{j l}\right) e_{i} e_{j}, \quad 1 \leq k<l \leq r
$$

Likewise, we note that

$$
\left(\sum_{i, j=1}^{r} e_{i}^{2} \otimes e_{j}^{2}\right)_{*}\left(e_{k} e_{l}\right)=0, \quad 1 \leq k<l \leq r
$$

and that

$$
\left(e_{i}^{2} \otimes e_{i}^{2}\right)_{*} e_{k} e_{l}=0, \quad 1 \leq k<l \leq r .
$$

From these, we see that the right-hand side of (18) acts as the identity on the sub-space of $\mathrm{H}^{4}(F, \mathbb{Q})$ spanned by $\left\{e_{k} e_{l}: 1 \leq k<l \leq r\right\}$. Now we consider the action of the right-hand side of (18) on $e_{k}^{2}$. First we note that

$$
\left(e_{i} e_{j} \otimes e_{i} e_{j}\right)_{*} e_{k}^{2}=0
$$

for all $1 \leq i<j \leq r$ and all $1 \leq k \leq r$, and also that

$$
\left(e_{i}^{2} \otimes e_{i}^{2}\right)_{*} e_{k}^{2}= \begin{cases}c_{F} e_{i}^{2}, & i \neq k \\ 3 c_{F} e_{i}^{2}, & i=k\end{cases}
$$


From the above equation, we get

$$
\left(\sum_{i=1}^{r} e_{i}^{2} \otimes e_{i}^{2}\right)_{*} e_{k}^{2}=3 c_{F} e_{k}^{2}+c_{F} \sum_{i \neq k} e_{i}^{2}=2 c_{F} e_{k}^{2}+c_{F} \sum_{i=1}^{r} e_{i}^{2} .
$$

Furthermore, we note that equation (17) implies that $\left(\sum_{i=1}^{r} e_{i}^{2}\right) e_{k}^{2}=(r+2) c_{F}[p t]$. It follows that

$$
\left(\sum_{i, j=1}^{r} e_{i}^{2} \otimes e_{j}^{2}\right)_{*} e_{k}^{2}=(r+2) c_{F} \sum_{i=1}^{r} e_{i}^{2} .
$$

Combining the above computations, we see that the right-hand side of (18) acts as the identity on the sub-space spanned by $\left\{e_{k}^{2}\right\}$. Thus it acts as the identity on the whole cohomology group $\mathrm{H}^{4}(F, \mathbb{Q})$.

Proof of Proposition 1.3. We may work with complex coefficients and hence use an orthonormal basis $\left\{e_{i}\right\}$ as above. Then statement (i) follows easily from Lemma 1.5 and Lemma 1.6.

Statement (ii) follows from the fact that $\mathfrak{B}^{k}$ lies in $\mathrm{H}^{2 k}(F) \otimes \mathrm{H}^{2 k}(F)$; see Lemma 1.4.

If we combine Lemma 1.4 with equations (19) and (20), we see that

$$
\left(\mathfrak{B}^{2}\right)_{*} e_{k} e_{l}=2 c_{F} e_{k} e_{l}, \quad 1 \leq k<l \leq r .
$$

Similarly, equations (21) and (22) give

$$
\left(\mathfrak{B}^{2}\right)_{*} e_{k}^{2}=2 c_{F} e_{k}^{2}+c_{F} \mathfrak{b}
$$

Note that $\mathfrak{b}=\sum e_{i}^{2}$, and therefore

$$
\left(\mathfrak{B}^{2}\right)_{*} \mathfrak{b}=(r+2) c_{F} \mathfrak{b} .
$$

Since by definition $\langle\mathfrak{b}\rangle^{\perp}$ is generated by $e_{i} e_{j}$ and $e_{i}^{2}-e_{j}^{2}$, we easily see that $\left(\mathfrak{B}^{2}\right)_{*}$ is multiplication by $2 c_{F}$ on $\langle\mathfrak{b}\rangle^{\perp}$. This proves statement (iii).

Statement (iv) can be proved using Lemma 1.4. For example take $e_{i}^{\vee} \in \mathrm{H}^{6}(F)$. Then we easily see that $\mathfrak{B}_{*} e_{i}^{\vee}=e_{i}$. Using the explicit expression for $\mathfrak{B}^{3}$ in Lemma 1.4, we get $\left(\mathfrak{B}^{3}\right)_{*} e_{i}=3(r+2) c_{F}^{2} e_{i}^{\vee}$. It follows that $\left(\mathfrak{B}^{3}\right)_{*} \circ(\mathfrak{B})_{*}$ is equal to $3(r+2) c_{F}^{2}$ on $\mathrm{H}^{6}(F, \mathbb{Q})$. The other equalities are proved similarly.

To prove $(v)$, we assume that $\mathfrak{C}$ satisfies the equation (12). We write $\mathfrak{C}=\sum a_{i j} e_{i} \otimes e_{j}$ and hence $\mathfrak{c}=\sum a_{i j} e_{i} e_{j}$. We define the transpose ${ }^{t} \mathfrak{C}:=\iota^{*} \mathfrak{C}=\sum a_{j i} e_{j} \otimes e_{i}$, where $\iota$ is the involution on $F \times F$ that switches the two factors. Let $A=\left(a_{i j}\right)$. Plugging in $\mathfrak{C}$ into equation (12) and taking the $\mathrm{H}^{4}(F) \otimes \mathrm{H}^{4}(F)$ component of the equation, we get

$$
\mathfrak{C}^{2}=2 c_{F}\left[\Delta_{F}\right]_{4,4}+\frac{1}{r+2} \mathfrak{c} \otimes \mathfrak{c}
$$

where []$_{4,4}$ means the $\mathrm{H}^{4}(F) \otimes \mathrm{H}^{4}(F)$-component. Given the explicit expression for $\left[\Delta_{F}\right]$ in Lemma 1.6, we can compare the coefficients of the two sides of (23). When we consider the coefficient of $e_{i}^{2} \otimes e_{i}^{2}$, we get

$$
\left(a_{i i}\right)^{2}=1-\frac{1}{r+2}+\frac{\left(a_{i i}\right)^{2}}{r+2}
$$

which implies that $a_{i i}= \pm 1$. Without loss of generality, we assume that $a_{i i}=1$ for $1 \leq i \leq r^{\prime}$ and $a_{i i}=-1$ for $r^{\prime}+1 \leq i \leq r$, where $0 \leq r^{\prime} \leq r$.

We first show that $\mathfrak{C}$ is symmetric, meaning $\mathfrak{C}={ }^{t} \mathfrak{C}$. Applying $\iota^{*}$ to equation (12) and taking the difference with the original equation, we get

$$
\mathfrak{C}^{2}-\left({ }^{t} \mathfrak{C}\right)^{2}=-\frac{1}{2}\left(\mathfrak{c}_{1}+\mathfrak{c}_{2}\right)\left(\mathfrak{C}-{ }^{t} \mathfrak{C}\right)
$$

Note that the left-hand side is an element in $\mathrm{H}^{4}(F) \otimes \mathrm{H}^{4}(F)$ and that $\mathfrak{c}_{1}\left(\mathfrak{C}-{ }^{t} \mathfrak{C}\right)\left(\right.$ resp. $\left.\mathfrak{c}_{2}\left(\mathfrak{C}-{ }^{t} \mathfrak{C}\right)\right)$ is an element in $\mathrm{H}^{6}(F) \otimes \mathrm{H}^{2}(F)$ (resp. $\mathrm{H}^{2}(F) \otimes \mathrm{H}^{6}(F)$ ). Hence we conclude that

$$
\mathfrak{C}^{2}=\left({ }^{t} \mathfrak{C}\right)^{2}, \quad \mathfrak{c}_{i}\left(\mathfrak{C}-{ }^{t} \mathfrak{C}\right)=0 .
$$

Comparing the coefficients of $e_{i}^{2} \otimes e_{j} e_{j^{\prime}}$ in $\mathfrak{C}^{2}$ and $\left({ }^{t} \mathfrak{C}\right)^{2}$, we get

$$
a_{i j} a_{i j^{\prime}}=a_{j i} a_{j^{\prime} i}, \quad \forall i, j, j^{\prime} .
$$

By taking $j^{\prime}=i$ in (25) and by noting that $a_{i i}= \pm 1$, we get $a_{i j}=a_{j i}$. Hence $\mathfrak{C}$ is symmetric. 
Now we have ${ }^{t} A=A$, and Lemma 1.4 yields

$$
\begin{aligned}
\mathfrak{c}_{1} \mathfrak{C} & =\sum a_{i j} a_{k l} e_{i} e_{j} e_{k} \otimes e_{l} \\
& =c_{F} \sum a_{i j} a_{k l}\left(\delta_{i j} e_{k}^{\vee}+\delta_{i k} e_{j}^{\vee}+\delta_{j k} e_{i}^{\vee}\right) \otimes e_{l} \\
& =c_{F} \sum_{i, j}\left(\sum_{k=1}^{r} a_{k k} a_{i j}+2 \sum_{k=1}^{r} a_{i k} a_{k j}\right) e_{i}^{\vee} \otimes e_{j} .
\end{aligned}
$$

Note that the left-hand side of equation (12) is purely in $\mathrm{H}^{4}(F) \otimes \mathrm{H}^{4}(F)$. Given Lemma 1.6 and considering the $\mathrm{H}^{6}(F) \otimes \mathrm{H}^{2}(F)$-component of the right-hand side of (12), we get

$$
2 A^{2}+(\operatorname{tr} A) A-(r+2) I=0 .
$$

Comparing the coefficients of $e_{i}^{2} \otimes e_{j}^{2}$ for $i \neq j$ in equation (23), we get

$$
\left(a_{i j}\right)^{2}=-\frac{1}{r+2}+\frac{a_{i i} a_{j j}}{r+2} .
$$

It follows that $a_{i j}=0$ for all $1 \leq i, j \leq r^{\prime}$ or $r^{\prime}+1 \leq i, j \leq r$ with $i \neq j$. Comparing the coefficients of $e_{i}^{2} \otimes e_{j} e_{k}$ for $j \neq k$ in equation (23), we get

$$
2 a_{i j} a_{i k}=\frac{2 a_{i i} a_{j k}}{r+2} .
$$

If $r^{\prime} \geq 2$, we take $1 \leq i \neq j \leq r^{\prime}$ and $r^{\prime}+1 \leq k \leq r$ in the above equation. Recalling that $a_{i j}=0$ it follows that $a_{j k}=0$ and hence we conclude that $A$ is diagonal. If $r^{\prime}=1$, we take $r^{\prime}+1 \leq i \neq j \leq r$ in the above equation, we can still conclude that $A$ is diagonal. So, in any case, $A$ is a diagonal matrix with \pm 1 coefficients in the diagonal. Then equation (26) implies that $A= \pm I$, namely $\mathfrak{C}= \pm \mathfrak{B}$.

Corollary 1.7. Let $F$ be a compact hyperkähler manifold of dimension 4 and let $\pi_{\text {hom }}^{i}$ be the class of the Künneth projector on $\mathrm{H}^{i}(F, \mathbb{Q})$ in $\mathrm{H}^{8}(F \times F, \mathbb{Q})$. Then

$$
\pi_{\text {hom }}^{0}=\frac{1}{c_{F} r(r+2)} \mathfrak{b}_{1}^{2}, \quad \pi_{\text {hom }}^{2}=\frac{1}{c_{F}(r+2)} \mathfrak{b}_{1} \mathfrak{B}, \quad \pi_{\text {hom }}^{6} \quad=\frac{1}{c_{F}(r+2)} \mathfrak{b}_{2} \mathfrak{B}, \quad \pi_{\text {hom }}^{8}=\frac{1}{c_{F} r(r+2)} \mathfrak{b}_{2}^{2} .
$$

Moreover, if $F$ satisfies the assumptions of Proposition 1.3, then equation (12) defines a Künneth decomposition of the diagonal and we have

$$
\pi_{\text {hom }}^{4}=\frac{1}{2 c_{F}}\left(\mathfrak{B}^{2}-\frac{1}{r+2} \mathfrak{b}_{1} \mathfrak{b}_{2}\right) .
$$

Proof. This follows from Lemma 1.4 and Lemma 1.5.

The cohomological Fourier transform is then easy to understand.

Proposition 1.8. Let $F$ be a compact hyperkähler manifold of dimension 4 and let $[\mathcal{F}]$ be the cohomological Fourier transform. Then

(i) $[\mathcal{F}] \circ[\mathcal{F}]=\frac{r(r+2) c_{F}^{2}}{8}$ on $\mathrm{H}^{0}(F, \mathbb{Q})$ and on $\mathrm{H}^{8}(F, \mathbb{Q})$;

(ii) $[\mathcal{F}] \circ[\mathcal{F}]=\frac{(r+2) c_{F}^{2}}{2}$ on $\mathrm{H}^{2}(F, \mathbb{Q})$ and on $\mathrm{H}^{6}(F, \mathbb{Q})$;

(iii) if $F$ satisfies the assumptions of Proposition 1.3, $[\mathcal{F}] \circ[\mathcal{F}]$ induces an eigenspace decomposition $\mathrm{H}^{4}(F, \mathbb{Q})=\langle\mathfrak{b}\rangle \oplus\langle\mathfrak{b}\rangle^{\perp}$, where $[\mathcal{F}] \circ[\mathcal{F}]=\left(\frac{(r+2)}{2} c_{F}\right)^{2}$ on $\langle\mathfrak{b}\rangle$ and $[\mathcal{F}] \circ[\mathcal{F}]=c_{F}^{2}$ on $\langle\mathfrak{b}\rangle^{\perp}$.

\section{The Fourier transform on the ChOW Groups of HyPERKÄHLER FourfoldS}

The aim of this section is to exhibit key properties that a cycle $L \in \mathrm{CH}^{2}(F \times F)$ representing the Beauville-Bogomolov class $\mathfrak{B}$ on a hyperkähler variety $F$ of $\mathrm{K} 3^{[2]}$-type should satisfy in order to induce a Fourier decomposition as in Theorem 2 on the Chow groups of $F$. The two main results here are Theorems $2.2 \& 2.4$; they reduce Theorem 2 to showing that $L$ satisfies hypotheses (6), (7), (8) and (9). 
Although in this paper we will be mostly considering hyperkähler varieties which are deformation equivalent to the Hilbert scheme of length-2 subschemes on a K3 surface, in which case by $[6,44]$ we have $c_{F}=1$ (so that the Fujiki constant is equal to 3 ) and $r=23$, we first formulate a more general version of Conjecture 1.

Conjecture 2.1. Let $F$ be a projective hyperkähler manifold of dimension 4 whose cohomology ring is generated by $\mathrm{H}^{2}(F, \mathbb{Q})$. Let $r=\operatorname{dim} \mathrm{H}^{2}(F, \mathbb{Q})$. Then there exists a canonical cycle $L \in \mathrm{CH}^{2}(F \times F)$ with cohomology class $\mathfrak{B} \in \mathrm{H}^{4}(F \times F, \mathbb{Q})$ satisfying

$$
L^{2}=2 c_{F} \Delta_{F}-\frac{2}{r+2}\left(l_{1}+l_{2}\right) \cdot L-\frac{1}{r(r+2)}\left(2 l_{1}^{2}-r l_{1} l_{2}+2 l_{2}^{2}\right) \quad \in \mathrm{CH}^{4}(F \times F),
$$

where by definition we have set $l:=\iota_{\Delta}^{*} L$ and $l_{i}:=p_{i}^{*} l$.

Theorem 2.2. Let $F$ be a hyperkähler variety of $\mathrm{K}^{[2]}$-type. Assume that there exists a cycle $L \in$ $\mathrm{CH}^{2}(F \times F)$ as in Conjecture 1 and assume moreover that

$$
\begin{gathered}
L_{*} l^{2}=0 ; \\
L_{*}\left(l \cdot L_{*} \sigma\right)=25 L_{*} \sigma \text { for all } \sigma \in \mathrm{CH}^{4}(F) .
\end{gathered}
$$

Then the action of $\left(L^{2}\right)_{*}$ on $\mathrm{CH}^{*}(F)$ diagonalizes. Precisely, for $\lambda \in \mathbb{Q}$, writing

$$
\Lambda_{\lambda}^{i}:=\left\{\sigma \in \mathrm{CH}^{i}(F):\left(L^{2}\right)_{*} \sigma=\lambda \sigma\right\}
$$

we have

$$
\begin{aligned}
& \mathrm{CH}^{4}(F)=\Lambda_{0}^{4} \oplus \Lambda_{2}^{4}, \text { with } \Lambda_{2}^{4}=\mathrm{F}^{4} \mathrm{CH}^{4}(F):=\operatorname{ker}\left\{L_{*}: \mathrm{CH}^{4}(F)_{\text {hom }} \rightarrow \mathrm{CH}^{2}(F)\right\} ; \\
& \mathrm{CH}^{3}(F)=\Lambda_{0}^{3} \oplus \Lambda_{2}^{3}, \text { with } \Lambda_{2}^{3}=\mathrm{CH}^{3}(F)_{\text {hom }} ; \\
& \mathrm{CH}^{2}(F)=\Lambda_{25}^{2} \oplus \Lambda_{2}^{2} \oplus \Lambda_{0}^{2}, \text { with } \Lambda_{25}^{2}=\langle l\rangle \text { and } \Lambda_{0}^{2}=L_{*} \mathrm{CH}^{4}(F) \subseteq \mathrm{CH}^{2}(F)_{\text {hom }} ; \\
& \mathrm{CH}^{1}(F)=\Lambda_{0}^{1} \\
& \mathrm{CH}^{0}(F)=\Lambda_{0}^{0} .
\end{aligned}
$$

Moreover, $l \cdot: \Lambda_{0}^{2} \rightarrow \mathrm{CH}^{4}(F)$ maps $\Lambda_{0}^{2}$ isomorphically onto $\left(\Lambda_{0}^{4}\right)_{\mathrm{hom}}$ with inverse given by $\frac{1}{25} L_{*}$ and $l \cdot: \Lambda_{0}^{1} \rightarrow \mathrm{CH}^{3}(F)$ maps $\Lambda_{0}^{1}$ isomorphically onto $\Lambda_{0}^{3}$ with inverse given by $\frac{1}{25} L_{*}$. We also have the natural inclusion $l \cdot \Lambda_{2}^{2} \subseteq \Lambda_{2}^{4}$.

Proof. Let us first remark that, by the projection formula, we have that for all $\sigma \in \mathrm{CH}^{*}(F)$ and all $i, j, k \geq 0$

$$
\left(l_{1}^{i} \cdot l_{2}^{j} \cdot L^{k}\right)_{*} \sigma=l^{j} \cdot\left(L^{k}\right)_{*}\left(l^{i} \cdot \sigma\right) .
$$

The following lemma will be used throughout the proof of the proposition.

Lemma 2.3. Assume that there exists $L \in \mathrm{CH}^{2}(F \times F)$ as in Conjecture 1 satisfying hypothesis (7). Then

$$
\left(L^{k}\right)_{*}\left(l^{j}\right)=0 \text { if } k+2 j \neq 4, \text { and }\left(L^{0}\right)_{*} l^{2}=23 \cdot 25[F],\left(L^{2}\right)_{*} l=25 l,\left(L^{4}\right)_{*}[F]=3 l^{2} .
$$

Proof of Lemma 2.3. Following directly from the cohomological description of $L$ are the following identities: $\left(L^{0}\right)_{*} l^{2}=23 \cdot 25 l^{0}$ and $L_{*} l^{j}=0$ for $j=0,1$. Now, using (6), we have

$$
\left(L^{2}\right)_{*} l^{j}=2 l^{j}+\left\{\begin{array}{lll}
-\frac{2}{23 \cdot 25}\left(L^{0}\right)_{*} l^{2} & =0 & \text { if } j=0 \\
+\frac{1}{25} l \cdot\left(L^{0}\right)_{*} l^{2} & =25 l & \text { if } j=1 \\
-\frac{2}{23 \cdot 25} l^{2} \cdot\left(L^{0}\right)_{*} l^{2} & =0 & \text { if } j=2 .
\end{array}\right.
$$

Because $\Delta_{F} \cdot L=\left(\iota_{\Delta}\right)_{*} \iota_{\Delta}^{*} L=\left(\iota_{\Delta}\right)_{*} l$, where $\iota_{\Delta}: F \rightarrow F \times F$ is the diagonal embedding, we have after intersecting (6) with $L$ :

which yields

$$
L^{3}=2\left(\iota_{\Delta}\right)_{*} l-\frac{2}{25}\left(l_{1}+l_{2}\right) \cdot L^{2}-\frac{1}{23 \cdot 25}\left(2 l_{1}^{2}-23 l_{1} l_{2}+2 l_{2}^{2}\right) \cdot L,
$$

$$
\left(L^{3}\right)_{*} l^{j}=2 l^{j+1}-\frac{2}{25}\left(L^{2}\right)_{*}\left(l^{j+1}\right)-\frac{2}{25} l \cdot\left(L^{2}\right)_{*}\left(l^{j}\right)=0 \text { for all } j .
$$


Finally, intersecting (6) with $L^{2}$ gives

$$
L^{4}=2\left(\iota_{\Delta}\right)_{*} l^{2}-\frac{2}{25}\left(l_{1}+l_{2}\right) \cdot L^{3}-\frac{1}{23 \cdot 25}\left(2 l_{1}^{2}-23 l_{1} l_{2}+2 l_{2}^{2}\right) \cdot L^{2},
$$

which, combined with the previous computations, yields the relation $\left(L^{4}\right)_{*} l^{0}=3 l^{2}$.

The decompositions induced by the action $L^{2}$ on the Chow groups of $F$ are established case by case.

Step 1: $\mathrm{CH}^{4}(F)=\Lambda_{2}^{4} \oplus \Lambda_{0}^{4}$, with $\Lambda_{2}^{4}=\mathrm{F}^{4} \mathrm{CH}^{4}(F)$.

Let $\sigma \in \mathrm{CH}^{4}(F)$ and denote $\tau:=l \cdot L_{*} \sigma$. Note that the statement (ii) of Proposition 1.3 implies that $L_{*} \sigma$ is homologically trivial and hence $\operatorname{deg}(\tau)=0$. Equation (6) applied to $\sigma$ gives

$$
\left(\left(L^{2}\right)_{*}-2\right) \sigma=-\frac{2}{25} l \cdot L_{*} \sigma-\frac{2 \operatorname{deg}(\sigma)}{23 \cdot 25} l^{2}=-\frac{2}{25} \tau-\frac{2 \operatorname{deg}(\sigma)}{23 \cdot 25} l^{2}
$$

On the one hand, by condition (8) we have $l \cdot L_{*} \tau=25 \tau$ so that

$$
\left(L^{2}\right)_{*} \tau=2 \tau-\frac{2}{25} l \cdot L_{*} \tau-\frac{2 \operatorname{deg}(\tau)}{23 \cdot 25} l^{2}=2 \tau-\frac{2}{25} \cdot 25 \tau=0 .
$$

On the other hand, we have $\left(L^{2}\right)_{*} l^{2}=0$ by Lemma 2.3 .

Applying $\left(L^{2}\right)_{*}$ to equation (31) thus gives

$$
\left(L^{2}\right)_{*}\left(\left(L^{2}\right)_{*}-2\right) \sigma=0,
$$

which yields the required eigenspace decomposition for $\mathrm{CH}^{4}(F)$.

Let us now show that $\Lambda_{2}^{4}=\mathrm{F}^{4} \mathrm{CH}^{4}(F)$. If $\sigma \in \mathrm{F}^{4} \mathrm{CH}^{4}(F)$, then $\tau=l \cdot L_{*} \sigma=0$. Hence we get $\left(L^{2}\right)_{*} \sigma=2 \sigma$. It follows that

$$
\mathrm{F}^{4} \mathrm{CH}^{4}(F) \subseteq \Lambda_{2}^{4} .
$$

Conversely, consider $\sigma \in \Lambda_{2}^{4}$. Then equation (31) implies

$$
0=-\frac{2}{25} \tau-\frac{2 \operatorname{deg}(\sigma)}{23 \cdot 25} l^{2}
$$

Since $\tau$ is homologically trivial, we get $\operatorname{deg}(\sigma)=0$, which further implies $\tau=0$. Then by condition (8) we have $25 L_{*} \sigma=L_{*} \tau=0$ which means that $L_{*} \sigma=0$. Hence we have

$$
\Lambda_{2}^{4} \subseteq \mathrm{F}^{4} \mathrm{CH}^{4}(F) .
$$

Finally, note that, as an immediate consequence of equation $(31)$ and of the fact that $\operatorname{deg}\left(\left(L^{2}\right)_{*} \sigma\right)=0$, we have

$$
\left(\Lambda_{0}^{4}\right)_{\mathrm{hom}}=\left\{\sigma \in \mathrm{CH}^{4}(F): l \cdot L_{*} \sigma=25 \sigma\right\} .
$$

Step 2 : $\mathrm{CH}^{3}(F)=\Lambda_{2}^{3} \oplus \Lambda_{0}^{3}$, where $\Lambda_{2}^{3}=\mathrm{CH}^{3}(F)_{\text {hom }}$.

Consider a divisor $\sigma \in \mathrm{CH}^{3}(F)$. Equation (6) applied to $\sigma$ gives

$$
\left(\left(L^{2}\right)_{*}-2\right) \sigma=-\frac{2}{25} l \cdot L_{*} \sigma
$$

Note that $L_{*} \sigma$ is a divisor, and recall from Corollary 1.7 that $L_{*}$ induces an isomorphism $\mathrm{H}^{6}(F, \mathbb{Q}) \stackrel{\simeq}{\longrightarrow}$ $\mathrm{H}^{2}(F, \mathbb{Q})$ with inverse given by intersecting with $\frac{1}{25} l$. Because $\mathrm{CH}^{1}(F)$ injects into $\mathrm{H}^{2}(F, \mathbb{Q})$ via the cycle class map, we obtain that $L_{*} \sigma=0$ if and only if $\sigma$ is homologically trivial. In other words, we have

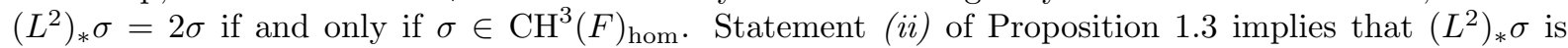
homologically trivial for all $\sigma \in \mathrm{CH}^{3}(F)$. Thus

$$
\left(\left(L^{2}\right)_{*}-2\right)\left(L^{2}\right)_{*} \sigma=0
$$

which yields the required eigenspace decomposition. Note for future reference that

$$
\Lambda_{0}^{3}=\left\{\sigma \in \mathrm{CH}^{3}(F): l \cdot L_{*} \sigma=25 \sigma\right\} .
$$

Step 3: $\mathrm{CH}^{2}(F)=\Lambda_{25}^{2} \oplus \Lambda_{2}^{2} \oplus \Lambda_{0}^{2}$, with $\Lambda_{25}^{2}=\langle l\rangle, \Lambda_{0}^{2}=L_{*} \mathrm{CH}^{4}(F)=L_{*}\left(\Lambda_{0}^{4}\right)_{\text {hom }}$. Furthermore, we have $\mathrm{CH}^{2}(F)_{\text {hom }}=\left(\Lambda_{2}^{2}\right)_{\text {hom }} \oplus \Lambda_{0}^{2}$. 
Let $\sigma \in \mathrm{CH}^{2}(F)$ and denote $\tau:=L_{*}(l \cdot \sigma)$. We know from (ii) of Proposition 1.3 that $L_{*} \sigma=0$ and that $\tau$ is homologically trivial. Equation (6), together with the identity $L_{*} l=0$, then gives

$$
\left(\left(L^{2}\right)_{*}-2\right) \sigma=-\frac{2}{25} L_{*}(l \cdot \sigma)+\frac{1}{25}\left(\int_{F}[l] \cup[\sigma]\right) l .
$$

In particular, equation (6) applied to $\tau$ gives

$$
\left(\left(L^{2}\right)_{*}-2\right) \tau=-\frac{2}{25} L_{*}(l \cdot \tau)=-\frac{2}{25} L_{*}\left(l \cdot L_{*}(l \cdot \sigma)\right)=-\frac{2}{25}\left(25 L_{*}(l \cdot \sigma)\right)=-2 \tau,
$$

where the third equality is hypothesis $(8)$. Thus $\left(L^{2}\right)_{*} \tau=0$. We then apply $\left(L^{2}\right)_{*}$ to equation (34) and get, in view of the identity $\left(L^{2}\right)_{*} l=25 l$ of Lemma 2.3 ,

$$
\left(L^{2}\right)_{*}\left(\left(L^{2}\right)_{*}-2\right) \sigma=\left(\int_{F}[l] \cup[\sigma]\right) l, \quad \forall \sigma \in \mathrm{CH}^{2}(F) .
$$

We then apply $\left(\left(L^{2}\right)_{*}-25\right)$ to the above equation and get

$$
\left(L^{2}\right)_{*}\left(\left(L^{2}\right)_{*}-2\right)\left(\left(L^{2}\right)_{*}-25\right) \sigma=0, \quad \forall \sigma \in \mathrm{CH}^{2}(F),
$$

which yields the required eigenspace decomposition.

It is worth noting that (35) implies that

$$
\left(L^{2}\right)_{*}\left(\left(L^{2}\right)_{*}-2\right) \sigma=0, \quad \forall \sigma \in \mathrm{CH}^{2}(F)_{\text {hom }} .
$$

Let us characterize the eigenspaces $\Lambda_{25}^{2}, \Lambda_{2}^{2}$ and $\Lambda_{0}^{2}$. First we show that $\Lambda_{25}^{2}$ is spanned by $l$. If $\sigma \in \Lambda_{25}^{2}$, then we have

$$
23 \sigma=\left(\left(L^{2}\right)_{*}-2\right) \sigma=-\frac{2}{25} \tau+\frac{1}{25}\left(\int_{F}[l] \cup[\sigma]\right) l .
$$

Since $\tau$ is homologically trivial, we see that the homology class $[\sigma]$ of $\sigma$ is a multiple of the homology class of $l$. Consequently, there exists a rational number $a$ such that $\sigma^{\prime}=\sigma-a l$ is homologically trivial and hence, by equation (36), we get

$$
\left(L^{2}\right)_{*}\left(\left(L^{2}\right)_{*}-2\right) \sigma^{\prime}=0
$$

Since $\sigma^{\prime} \in \Lambda_{25}^{2}$, the above equation implies $\sigma^{\prime}=0$, namely $\sigma=a l$. This proves that $\Lambda_{25}^{2}=\langle l\rangle$.

Identity (34), together with Proposition 1.3(iii), implies that $\sigma \in \mathrm{CH}^{2}(F)$ satisfies $\left(L^{2}\right)_{*} \sigma=2 \sigma$ if and only if $L_{*}(l \cdot \sigma)=0$ and $\operatorname{deg}(l \cdot \sigma)=0$. Thus, since $\Lambda_{2}^{4}=\mathrm{F}^{4} \mathrm{CH}^{4}(F)$, we get

$$
\Lambda_{2}^{2}=\left\{\sigma \in \mathrm{CH}^{2}(F):\left(L^{2}\right)_{*}(l \cdot \sigma)=2 l \cdot \sigma\right\} .
$$

In particular, this implies the inclusion $l \cdot \Lambda_{2}^{2} \subseteq \Lambda_{2}^{4}$.

Finally, we give a description of the space $\Lambda_{0}^{2}$. Statement (iii) of Proposition 1.3 implies that 0 is not an eigenvalue of $\left(L^{2}\right)_{*}$ acting on the cohomology group $\mathrm{H}^{4}(F, \mathbb{Q})$. Hence all the elements of $\Lambda_{0}^{2}$ are homologically trivial. Thus, if $\sigma \in \Lambda_{0}^{2}$, then we have

$$
-2 \sigma=\left(\left(L^{2}\right)_{*}-2\right) \sigma=-\frac{2}{25} L_{*}(l \cdot \sigma) .
$$

It follows that $\sigma=\frac{1}{25} L_{*}(l \cdot \sigma)$, namely that $\Lambda_{0}^{2} \subseteq L_{*} \mathrm{CH}^{4}(F)$. Consider now $\sigma \in L_{*} \mathrm{CH}^{4}(F)$. Note that $\sigma$ is homologically trivial by statement (ii) of Proposition 1.3. By condition (8), we see that

$$
L_{*}(l \cdot \sigma)=25 \sigma .
$$

Hence it follows that

$$
\left(\left(L^{2}\right)_{*}-2\right) \sigma=-\frac{2}{25} L_{*}(l \cdot \sigma)+\frac{1}{25}\left(\int_{F}[l] \cup[\sigma]\right) l=-2 \sigma,
$$

which further gives $\sigma \in \Lambda_{0}^{2}$. Thus we have the inclusion $L_{*} \mathrm{CH}^{4}(F) \subseteq \Lambda_{0}^{2}$. The above arguments establish the equality

$$
\Lambda_{0}^{2}=L_{*} \mathrm{CH}^{4}(F) .
$$

Actually, since $L_{*} l^{2}=0$ and $L_{*} \Lambda_{2}^{4}=0$, we have

$$
\Lambda_{0}^{2}=L_{*}\left(\Lambda_{0}^{4}\right)_{\text {hom }}
$$


The identity (32) then implies that $L_{*}:\left(\Lambda_{0}^{4}\right)_{\text {hom }} \rightarrow \Lambda_{0}^{2}$ is an isomorphism with inverse $\frac{1}{25} l \cdot: \Lambda_{0}^{2} \rightarrow$ $\left(\Lambda_{0}^{4}\right)_{\text {hom }}$.

Step 4 : $\mathrm{CH}^{1}(F)=\Lambda_{0}^{1}$. Indeed, $\mathrm{CH}^{1}(F)$ injects into $\mathrm{H}^{2}(F, \mathbb{Q})$ by the cycle class map and $L^{2}$ acts as zero on $\mathrm{H}^{2}(F, \mathbb{Q})$ by Proposition $1.3(i i)$.

Step $5: \mathrm{CH}^{0}(F)=\Lambda_{0}^{0}$. This is obvious from Lemma 2.3 .

Theorem 2.4. Let $F$ be a hyperkähler variety of $\mathrm{K}^{[2]}$-type. Assume that there exists $L \in \mathrm{CH}^{2}(F \times F)$ as in Conjecture 1 satisfying conditions (7) and (8). For $\lambda \in \mathbb{Q}$, denote

$$
W_{\lambda}^{i}:=\left\{\sigma \in \mathrm{CH}^{i}(F): \mathcal{F} \circ \mathcal{F}(\sigma)=\lambda \sigma\right\} .
$$

Then, with the notations of Theorem 2 and Theorem 2.2, we have

$$
\begin{aligned}
\mathrm{CH}^{4}(F)_{0} & =W_{\frac{23 \cdot 25}{8}}^{4}=\left\langle l^{2}\right\rangle, \mathrm{CH}^{4}(F)_{2}=W_{\frac{25}{2}}^{4}=\left(\Lambda_{0}^{4}\right)_{\text {hom }}, \mathrm{CH}^{4}(F)_{4}=W_{1}^{4}=\Lambda_{2}^{4} ; \\
\mathrm{CH}^{3}(F)_{0} & =W_{\frac{25}{2}}^{3}=\Lambda_{0}^{3}, \mathrm{CH}^{3}(F)_{2}=W_{1}^{3}=\Lambda_{2}^{3} ; \\
W_{\left(\frac{25}{2}\right)^{2}}^{2} & =\Lambda_{25}^{2}=\langle l\rangle, W_{1}^{2}=\Lambda_{2}^{2}, \mathrm{CH}^{2}(F)_{2}=W_{\frac{25}{2}}^{2}=\Lambda_{0}^{2} .
\end{aligned}
$$

Assume moreover that $L$ satisfies the following condition :

$$
\left(L^{2}\right)_{*}\left(l \cdot\left(L^{2}\right)_{*} \sigma\right)=0 \text { for all } \sigma \in \mathrm{CH}^{2}(F) .
$$

Then

$$
\mathrm{CH}^{2}(F)_{0}=\Lambda_{25}^{2} \oplus \Lambda_{2}^{2}
$$

Proof. All is needed is to understand the actions of $\left(L^{s}\right)_{*}$ and $\left(L^{r}\right)_{*}\left(L^{s}\right)_{*}$ on the pieces $\Lambda_{\lambda}^{i}$. Clearly, for dimension reasons, $\left(L^{s}\right)_{*} \Lambda_{\lambda}^{i}=0$ either if $i<4-2 s$ or $i>8-2 s$.

First, we note that the action of $L^{2}$ does not completely decompose $\mathrm{CH}^{4}(F)$. The correspondence $L^{0}=[F \times F]$ clearly acts as zero on $\mathrm{CH}^{4}(F)_{\text {hom }}$. In particular $L^{0}$ acts as zero on $\Lambda_{2}^{4}$. By Lemma $2.3, F$ satisfies condition (28). Thus $L^{4} \circ L^{0}$ acts as multiplication by $3 \cdot 23 \cdot 25$ on $l^{2}$ and $\left(L^{2}\right)_{*} l^{2}=0$. It follows that the action of $L^{4} \circ L^{0}$ commutes with the action of $L^{2}$ on $\mathrm{CH}^{4}(F)$. Therefore, $\Lambda_{0}^{4}$ further splits into

$$
\Lambda_{0}^{4}=\left\langle l^{2}\right\rangle \oplus\left(\Lambda_{0}^{4}\right)_{\text {hom }} .
$$

Now by condition (7) we have $L_{*} l^{2}=0$, and by Theorem 2.2 we have $L_{*} \Lambda_{0}^{2}=0$. We claim that $L^{3} \circ L$ acts by multiplication by $3 \cdot 25$ on $\left(\Lambda_{0}^{4}\right)_{\text {hom }}$. Recall that we have equation (29) for the expression of $L^{3}$. Thus, having in mind that $L_{*} \sigma$ is homologically trivial for $\sigma \in \mathrm{CH}^{4}(F)$,

$$
\left(L^{3}\right)_{*}\left(L_{*} \sigma\right)=2 l \cdot L_{*} \sigma-\frac{2}{25}\left(l \cdot\left(L^{2}\right)_{*} L_{*} \sigma+\left(L^{2}\right)_{*}\left(l \cdot L_{*} \sigma\right)\right)+\frac{1}{25} l \cdot L_{*}\left(l \cdot L_{*} \sigma\right), \quad \text { for all } \sigma \in \mathrm{CH}^{4}(F) .
$$

Condition (8) gives $l \cdot L_{*}\left(l \cdot L_{*} \sigma\right)=25 l \cdot L_{*} \sigma$ and Theorem 2.2 gives $L_{*} \sigma \in \Lambda_{0}^{2}$, i.e., $\left(L^{2}\right)_{*} L_{*} \sigma=0$. Hence

$$
\left(L^{3}\right)_{*}\left(L_{*} \sigma\right)=3 l \cdot L_{*} \sigma-\frac{2}{25}\left(L^{2}\right)_{*}\left(l \cdot L_{*} \sigma\right), \quad \text { for all } \sigma \in \mathrm{CH}^{4}(F) .
$$

The claim then follows from the identity (32) which stipulates that $l \cdot L_{*} \sigma=25 \sigma$ for all $\sigma \in\left(\Lambda_{0}^{4}\right)_{\text {hom }}$.

Since $\Lambda_{2}^{3}=\mathrm{CH}^{3}(F)_{\text {hom }}$, it is clear that $L_{*} \Lambda_{2}^{3}=0$. Consider $\sigma \in \Lambda_{0}^{3}$. Then Theorem 2.2 gives $L_{*} \sigma \in \Lambda_{0}^{1}$ and $l \cdot L_{*} \sigma=25 \sigma$. Equation (29) applied to $L_{*} \sigma$ yields

$$
\left(L^{3}\right)_{*} L_{*} \sigma=2 l \cdot L_{*} \sigma+\frac{1}{25} l \cdot L_{*}\left(l \cdot L_{*} \sigma\right)=3 \cdot 25 \sigma .
$$

Condition (7), via Lemma 2.3, gives $\left(L^{k}\right)_{*} \Lambda_{2}^{25}=0$ unless $k=2$, and Proposition 1.3 gives $L_{*} \mathrm{CH}^{2}(F)=$ 0 . Recall that by Theorem 2.2 we have $\Lambda_{0}^{2}=L_{*}\left(\Lambda_{0}^{4}\right)_{\text {hom }}$. Consider $\sigma \in \Lambda_{0}^{2}$ and $\sigma_{0} \in\left(\Lambda_{0}^{4}\right)_{\text {hom }}$ such that $\sigma=L_{*} \sigma_{0}$. Equation (29), together with equation (32), then gives $\left(L^{3}\right)_{*} \sigma=3 \cdot 25 \sigma_{0}$, so that

$$
L_{*}\left(L^{3}\right)_{*} \sigma=3 \cdot 25 \sigma .
$$


Let us now consider an element $\sigma \in \Lambda_{2}^{2}$. According to (37), $\sigma \in \Lambda_{2}^{2}$ if and only if $l \cdot \sigma \in \Lambda_{2}^{4}$; and according to Theorem 2.2, $l \cdot \sigma \in \Lambda_{2}^{4}$ only if $L_{*}(l \cdot \sigma)=0$. Therefore applying (29) to $\sigma$ yields

$$
\left(L^{3}\right)_{*} \sigma=2 l \cdot \sigma+\frac{2}{25} l \cdot\left(L^{2}\right)_{*} \sigma+\frac{2}{25}\left(L^{2}\right)_{*}(l \cdot \sigma)+\frac{1}{25} l \cdot L_{*}(l \cdot \sigma)=\frac{58}{25} l \cdot \sigma .
$$

It immediately follows that

$$
L_{*}\left(L^{3}\right)_{*} \sigma=0, \quad \text { for all } \sigma \in \Lambda_{2}^{2} .
$$

Note that

$$
\text { Condition (9) is equivalent to } l \cdot \Lambda_{2}^{2}=0 \text {. }
$$

Indeed, consider $\sigma \in \Lambda_{2}^{2}$. On the one hand, (37) gives $l \cdot \sigma \in \Lambda_{2}^{4}$. On the other hand, condition (9) gives $\left(L^{2}\right)_{*}(l \cdot \sigma)=0$, i.e., $l \cdot \sigma \in \Lambda_{0}^{4}$. But, by Theorem $2.2, \mathrm{CH}^{4}(F)=\Lambda_{0}^{4} \oplus \Lambda_{2}^{4}$. Hence $l \cdot \sigma=0$.

Thus, assuming (9), (38) gives $\left(L^{3}\right)_{*} \sigma=0$ for all $\sigma \in \Lambda_{2}^{2}$. This yields the identity $\mathrm{CH}^{2}(F)_{0}=\Lambda_{25}^{2} \oplus \Lambda_{2}^{2}$.

Finally, both $\mathrm{CH}^{1}(F)$ and $\mathrm{CH}^{0}(F)$ inject by the cycle class map into $\mathrm{H}^{2}(F, \mathbb{Q})$ and $\mathrm{H}^{0}(F, \mathbb{Q})$ respectively, so that the proof here reduces to Proposition 1.3.

\section{The Fourier DeCOMposition IS MOtiviC}

Let $F$ be a hyperkähler variety of $\mathrm{K} 3^{[2]}$-type. In this section, we show that, provided $F$ is endowed with a cycle $L \in \mathrm{CH}^{2}(F \times F)$ representing the Beauville-Bogomolov class $\mathfrak{B}$ satisfying (6), (7), (8) and (9), the induced Fourier decomposition of Theorem 2 arises from a Chow-Künneth decomposition of the diagonal.

Let $X$ be a smooth projective variety of dimension $d$ defined over a field $k$. Murre [39] conjectured the existence of mutually orthogonal idempotents $\pi^{0}, \ldots, \pi^{2 d}$ in the ring (for the composition law) of correspondences $\mathrm{CH}^{d}(X \times X)$ such that $\Delta_{X}=\pi^{0}+\ldots+\pi^{2 d} \in \mathrm{CH}^{d}(X \times X)$ and such that the cohomology class of $\pi^{j} \in \mathrm{H}^{2 d}(X \times X) \subseteq \operatorname{End}\left(\mathrm{H}^{*}(X)\right)$ is the projector on $\mathrm{H}^{j}(X)$. Here $\mathrm{H}^{j}(X)$ denotes the $\ell$ adic cohomology group $\mathrm{H}^{j}\left(X_{\bar{k}}, \mathbb{Q}_{\ell}\right)$. Such a decomposition of the diagonal is called a Chow-Künneth decomposition; it is a lift of the Künneth decomposition of id $\in \operatorname{End}\left(\mathrm{H}^{*}(X)\right)$ via the cycle class map $\mathrm{CH}^{d}(X \times X) \rightarrow \mathrm{H}^{2 d}(X \times X)$. Furthermore, Murre conjectured that any Chow-Künneth decomposition satisfies the following properties :

(B) $\pi_{*}^{i} \mathrm{CH}^{j}(X)=0$ for $i>2 j$ and for $i<j$;

(D) $\operatorname{ker}\left\{\pi_{*}^{2 j}: \mathrm{CH}^{j}(X) \rightarrow \mathrm{CH}^{j}(X)\right\}=\mathrm{CH}^{j}(X)_{\text {hom }}$.

If such a Chow-Künneth decomposition exists, we may define a descending filtration $\mathrm{F}^{\bullet}$ on $\mathrm{CH}^{j}(X)$ as follows :

$$
\mathrm{F}^{r} \mathrm{CH}^{j}(X):=\operatorname{ker}\left\{\left(\pi^{2 j}+\ldots+\pi^{2 j-r+1}\right)_{*}: \mathrm{CH}^{j}(X) \rightarrow \mathrm{CH}^{j}(X)\right\} .
$$

Thus $\mathrm{F}^{0} \mathrm{CH}^{j}(X)=\mathrm{CH}^{j}(X), \mathrm{F}^{1} \mathrm{CH}^{j}(X)=\mathrm{CH}^{j}(X)$ hom and $\mathrm{F}^{j+1} \mathrm{CH}^{j}(X)=0$. Murre further conjectured (C) The filtration $\mathrm{F}^{\bullet}$ does not depend on the choice of Chow-Künneth decomposition.

It is a theorem of Jannsen [32] that Murre's conjectures for all smooth projective varieties are equivalent to the conjectures of Bloch and Beilinson [12,13] ; see [32, Conjecture 2.1] for a formulation of these conjectures. Here we show that a hyperkähler fourfold that satisfies the assumptions of Theorems 2.2 and 2.4, e.g. the Hilbert scheme of length-2 subschemes on a K3 surface or the Fano scheme of lines on a smooth cubic fourfold, has a Chow-Künneth decomposition that satisfies (B) and for which the induced filtration on $\mathrm{CH}^{4}(F)$ is the one considered in (4). Note that the existence of a Chow-Künneth decomposition for the Hilbert scheme of length- $n$ subschemes on a surface follows directly from the existence of a Chow-Künneth decomposition for surfaces [38] and from the work of de Cataldo and Migliorini [18]. We will also give in Section 13 another way of constructing a Chow-Künneth decomposition for $S^{[2]}$. In the same spirit as Conjecture 3, all these Chow-Künneth decompositions for $S^{[2]}$ should agree provided that one starts with the Chow-Künneth decomposition for $S$ given by $\pi_{S}^{0}=\mathfrak{o}_{S} \times S, \pi_{S}^{4}=S \times \mathfrak{o}_{S}$ and $\pi_{S}^{2}=\Delta_{S}-\pi_{S}^{0}-\pi_{S}^{4}$, where $\mathfrak{o}_{S}$ is any point lying on a rational curve on $S$.

The following lemma relies on a technique initiated by Bloch and Srinivas [14]. 
Lemma 3.1. Let $X$ be a smooth projective variety over a field $k$ and let $\Omega$ be a universal domain containing $k$. If $f \in \mathrm{CH}^{\operatorname{dim} X}(X \times X)$ is a correspondence such that $\left(f_{\Omega}\right)_{*} \mathrm{CH}_{0}\left(X_{\Omega}\right)=0$, then there is a smooth projective variety $Y$ of dimension $\operatorname{dim} X-1$ and correspondences $g \in \mathrm{CH}^{\operatorname{dim} X}(Y \times X)$ and $h \in \mathrm{CH}_{\operatorname{dim} X}(X \times Y)$ such that $f=g \circ h$. If $\operatorname{dim} X=0$, then $f=0$.

Proof. The lemma is clear when $d:=\operatorname{dim} X=0$. Let $k(X)$ be the function field of $X$. The assumption that $\left(f_{\Omega}\right)_{*} \mathrm{CH}_{0}\left(X_{\Omega}\right)=0$ implies that $\left(f_{k(X)}\right)_{*} \mathrm{CH}_{0}\left(X_{k(X)}\right)=0$. Let $\eta$ be the generic point of $X$ seen as a $k(X)$-rational point. In particular, $\left(f_{k(X)}\right)_{*}[\eta]=0$. The 0 -cycle $\left(f_{k(X)}\right)_{*}[\eta] \in \mathrm{CH}_{0}(k(X) \times X)$ coincides with the restriction of $f$ along the map $\mathrm{CH}^{d}(X \times X) \rightarrow \mathrm{CH}_{0}(k(X) \times X)$ obtained as the direct limit, indexed by the non-empty open sub-sets $U$ of $X$, of the flat pull-back maps $\mathrm{CH}^{d}(X \times X) \rightarrow \mathrm{CH}^{d}(U \times X)$. Therefore, by the localization exact sequence for Chow groups, $f$ is supported on $D \times X$ for some divisor $D$ inside $X$. If $Y \rightarrow D$ is an alteration of $D$, then $f$ factors through $Y$.

Proposition 3.2. Let $X$ be a smooth projective variety over a field $k$ and let $\Omega$ be a universal domain containing $k$. If $f \in \mathrm{CH}^{\operatorname{dim} X}(X \times X)$ is a correspondence such that $\left(f_{\Omega}\right)_{*} \mathrm{CH}_{*}\left(X_{\Omega}\right)=0$, then $f$ is nilpotent. Precisely, if $N:=2^{\operatorname{dim} X}-1$, we have $f^{\circ N}=0$.

Proof. We proceed by induction on $\operatorname{dim} X$. If $\operatorname{dim} X=0$, then by Lemma $3.1 f=0$. Let's now assume that $\operatorname{dim} X>0$. By Lemma 3.1, there is a smooth projective variety $Y$ of dimension $\operatorname{dim} X-1$ and correspondences $g \in \mathrm{CH}^{\operatorname{dim} X}(Y \times X)$ and $h \in \mathrm{CH}_{\operatorname{dim} X}(X \times Y)$ such that $f=g \circ h$. The correspondence $(h \circ g \circ h \circ g)_{\Omega} \in \mathrm{CH}_{\operatorname{dim} Y}\left(Y_{\Omega} \times Y_{\Omega}\right)$ then acts as zero on $\mathrm{CH}_{*}\left(Y_{\Omega}\right)$. By induction, $h \circ g \circ h \circ g$ is nilpotent of index $2^{\operatorname{dim} Y}-1$. It immediately follows that $f$ is nilpotent of index $2 \cdot\left(2^{\operatorname{dim} Y}-1\right)+1=2^{\operatorname{dim} X}-1$.

Theorem 3.3. Let $F$ be a hyperkähler fourfold of $\mathrm{K}^{[2]}$-type that satisfies the assumptions of Theorems 2.2 and 2.4. Then $F$ has a Chow-Künneth decomposition $\left\{\pi^{0}, \pi^{2}, \pi^{4}, \pi^{6}, \pi^{8}\right\}$ that satisfies (B) and such that

$$
\pi_{*}^{s} \mathrm{CH}^{i}(F)=\mathrm{CH}^{i}(F)_{2 i-s} .
$$

Moreover, $\left\{\pi^{0}, \pi^{2}, \pi^{4}, \pi^{6}, \pi^{8}\right\}$ satisfies $(D)$ if and only if the cycle class map cl $: \mathrm{CH}^{2}(F) \rightarrow \mathrm{H}^{4}(F, \mathbb{Q})$ restricted to $\mathrm{CH}^{2}(F)_{0}$ is injective.

Proof. In view of Corollary 1.7, it is tempting to think that the identity (6) already gives a Chow-Künneth decomposition of the diagonal (and this would be a consequence of Conjecture 3 ; see Theorem 8.18). We cannot prove this but the arguments below consist in modifying the correspondences of Proposition 1.7 so as to turn them into mutually orthogonal idempotents modulo rational equivalence.

First we define $\pi^{0}=\frac{1}{23 \cdot 25} l_{1}^{2}$ and $\pi^{8}=\frac{1}{23 \cdot 25} l_{2}^{2}$. These are clearly orthogonal idempotents. Then we define

$$
p:=\frac{1}{25} L \cdot l_{2} \in \mathrm{CH}^{4}(F \times F) .
$$

The correspondence $p$ defines an idempotent in $\mathrm{H}^{8}(F \times F, \mathbb{Q})$, which is the Künneth projector onto $\mathrm{H}^{6}(F)$; see Corollary 1.7. Because $L_{*} l^{2}=0 \in \mathrm{CH}^{*}(F)$, we see that $p \circ \pi^{8}=\pi^{8} \circ p=0$. The action of $p_{*}$ on $\mathrm{CH}^{*}(F)$ is given by $p_{*} \sigma=l \cdot L_{*} \sigma$ and the action of $p^{*}$ is given by $p^{*} \sigma=L_{*}(l \cdot \sigma)$. The action of $p_{*}$ on $\mathrm{CH}^{i}(F)_{s}$ is zero unless $2 i-s=6$, in which case $p_{*}$ acts as the identity, and the action of $p^{*}$ on $\mathrm{CH}^{i}(F)_{s}$ is zero unless $2 i-s=2$, in which case $p^{*}$ acts as the identity ; see the proof of Proposition 2.2, especially the identities (32), (33) and (37) therein. Finally, we note that $p \circ{ }^{t} p$ factors through $L \circ L \in \mathrm{CH}^{0}(F \times F)$ which is zero because $L_{*} l^{2}=0$.

The above shows that the correspondence $p \circ p-p \in \mathrm{CH}^{4}(F \times F)$ acts as zero on $\mathrm{CH}^{*}(F)$. By Proposition 3.2, $p \circ p-p$ is nilpotent, say of index $N$. It follows that the image, denoted $A$, of the homomorphism of $\mathbb{Q}$-algebras $\mathbb{Q}[T] \rightarrow \mathrm{CH}^{4}(F \times F)$ which sends $T$ to $p$ is a quotient of $\mathbb{Q}[T] /\left(T^{N}(T-1)^{N}\right)$, in particular a commutative finite-dimensional $\mathbb{Q}$-algebra. Moreover, if we consider the reduced algebra, then $\bar{p} \in A / \operatorname{Nil}(A)$ is a projector. By Wedderburn's section theorem (cf. [5]), we can lift $\bar{p}$ to a genuine projector $q$ in $A$, which differs from $p$ by a nilpotent element $n \in A$. Since neither $p$ nor $1-p$ are nilpotent (they both define non-trivial projectors modulo homological equivalence), we remark that $n$ must factor through $p \circ p-p$. Thus $p$ and $q$ are homologically equivalent correspondences and, because $p \circ p-p$ acts as zero on $\mathrm{CH}^{*}(F)$, it is also apparent that $p_{*}$ and $q_{*}$ have the same action on $\mathrm{CH}^{*}(F)$. Thus $q$ is an idempotent whose cohomology class is the projector on $\mathrm{H}^{6}(M)$ and whose action $q_{*}$ on $\mathrm{CH}^{4}(F)$ is the projector with image $\mathrm{CH}^{4}(F)_{2}$ and kernel $\mathrm{CH}^{4}(F)_{0} \oplus \mathrm{CH}^{4}(F)_{4}$. 
We noted that $p \circ{ }^{t} p=0$. We also noted that $q=p+n$, where $n$ factors through $p \circ p-p$ and also commutes with $p$. Therefore $q \circ{ }^{t} q=0$. We then define

$$
\pi^{2}:={ }^{t} q \circ\left(1-\frac{1}{2} q\right) \quad \text { and } \quad \pi^{6}:=\left(1-\frac{1}{2}^{t} q\right) \circ q .
$$

The correspondences $\pi^{2}$ and $\pi^{6}$ are then clearly orthogonal idempotents in $\mathrm{CH}^{4}(F \times F)$. By Poincaré duality, we see that the classes modulo homological equivalence of $\pi^{2}$ and $\pi^{6}$ are $\pi_{\text {hom }}^{2}$ and $\pi_{\text {hom }}^{6}$, respectively. It is also apparent that $\pi_{*}^{0} \mathrm{CH}^{*}(F)=\mathrm{CH}^{0}(F)_{0}, \pi_{*}^{2} \mathrm{CH}^{*}(F)=\mathrm{CH}^{1}(F)_{0} \oplus \mathrm{CH}^{2}(F)_{2}$, $\pi_{*}^{6} \mathrm{CH}^{*}(F)=\mathrm{CH}^{3}(F)_{0} \oplus \mathrm{CH}^{4}(F)_{2}$, and $\pi_{*}^{8} \mathrm{CH}^{*}(F)=\mathrm{CH}^{4}(F)_{0}$.

We now define

$$
\pi^{4}:=\Delta_{F}-\left(\pi^{0}+\pi^{2}+\pi^{6}+\pi^{8}\right)
$$

It is then clear that $\left\{\pi^{0}, \pi^{2}, \pi^{4}, \pi^{6}, \pi^{8}\right\}$ defines a Chow-Künneth decomposition for $F$ which satisfies $\pi_{*}^{4} \mathrm{CH}^{*}(F)=\mathrm{CH}^{2}(F)_{0} \oplus \mathrm{CH}^{3}(F)_{2} \oplus \mathrm{CH}^{4}(F)_{4}$ as well as Murre's conjecture (B).

Finally, note that the correspondences ${ }^{t} p$ and ${ }^{t} q$ act the same on $\mathrm{CH}^{2}(F)$ hom : they project onto $\mathrm{CH}^{2}(F)_{2}$ along $\mathrm{CH}^{2}(F)_{0, \text { hom }}$. Moreover, $q$ acts as zero on $\mathrm{CH}^{2}(F)_{\text {hom }}$. Thus $\pi^{2}$ acts like ${ }^{t} p$ on $\mathrm{CH}^{2}(F)_{\text {hom }}$. By the above, we also have a decomposition $\mathrm{CH}^{2}(F)=\operatorname{im}\left\{\pi_{*}^{4}\right\} \oplus \operatorname{im}\left\{\pi_{*}^{2}\right\}=\operatorname{im}\left\{\pi_{*}^{4}\right\} \oplus \operatorname{ker}\left\{\pi_{*}^{4}\right\}$, where $\pi_{*}^{4}$ and $\pi_{*}^{2}$ are acting on $\mathrm{CH}^{2}(F)$. Therefore, Murre's conjecture (D) holds if and only if $\operatorname{ker}\left\{\pi_{*}^{4}\right\}=$ $\mathrm{CH}^{2}(F)_{\text {hom }}$ if and only if $\operatorname{im}\left\{\pi_{*}^{2}\right\}=\mathrm{CH}^{2}(F)_{\text {hom }}$ if and only if $\mathrm{CH}^{2}(F)_{0, \text { hom }}=0$.

Let us end this paragraph with the following proposition which gives evidence for the uniqueness up to sign of a cycle $L \in \mathrm{CH}^{2}(F \times F)$ satisfying the quadratic equation (6).

Proposition 3.4. Let $F$ be a hyperkähler fourfold of $\mathrm{K}^{[2]}$-type endowed with a cycle $L \in \mathrm{CH}^{2}(F \times$ $F)$ representing the Beauville-Bogomolov class $\mathfrak{B}$. Assume that $L$ is symmetric, that is ${ }^{t} L=L$, and satisfies (6), (7), (8) and (9). Assume that the Fourier decomposition of Theorem 2.4 satisfies $\mathrm{CH}^{2}(F)_{2}$. $\mathrm{CH}^{2}(F)_{2} \subseteq \mathrm{CH}^{4}(F)_{4}$. Assume the Bloch-Beilinson conjectures. Then $L$ is the unique symmetric cycle in $\mathrm{CH}^{2}(F \times F)$ representing $\mathfrak{B}$ that satisfies the quadratic equation (6).

Proof. Let $L$ be as in Theorem 1 and let $L+E$ be another cycle representing $\mathfrak{B}$ that satisfies the quadratic equation (6). In particular, $E$ is homologically trivial. Writing $l:=\iota_{\Delta}^{*} L, l_{i}:=p_{i}^{*} l, \varepsilon:=\iota_{\Delta}^{*} E$ and $\varepsilon_{i}:=p_{i}^{*} \varepsilon$, where $\iota_{\Delta}: F \rightarrow F \times F$ is the diagonal embedding and $p_{i}: F \times F \rightarrow F$ are the projections, we have

$$
(L+E)^{2}=2 \Delta_{F}-\frac{2}{25}\left(l_{1}+\varepsilon_{1}+l_{2}+\varepsilon_{2}\right) \cdot(L+E)-\frac{2}{23 \cdot 25}\left(\left(l_{1}+\varepsilon_{1}\right)^{2}+\left(l_{2}+\varepsilon_{2}\right)^{2}\right)+\frac{1}{25}\left(l_{1}+\varepsilon_{1}\right) \cdot\left(l_{2}+\varepsilon_{2}\right) .
$$

Subtracting the equation satisfied by $L$ and pulling back along the diagonal, we obtain

$$
\varepsilon^{2}+2 \varepsilon \cdot l=0 \in \mathrm{CH}^{4}(F) .
$$

Since $E$ is homologically trivial, $\varepsilon$ is also homologically trivial. Assuming the Bloch-Beilinson conjectures, we get by Theorem 3.3 that $\varepsilon \in \mathrm{CH}^{2}(F)_{2}$. Therefore we have on the one hand $\varepsilon^{2} \in \mathrm{CH}^{4}(F)_{4}$ by assumption, and on the other hand $\varepsilon \cdot l \in \mathrm{CH}^{4}(F)_{2}$ by Theorem 2.4. It immediately follows that $\varepsilon^{2}=\varepsilon \cdot l=0$. By Theorem 2.2, $l \cdot: \mathrm{CH}^{2}(F)_{2} \rightarrow \mathrm{CH}^{4}(F)$ is injective. We conclude that $\varepsilon=0$.

Let us now write $\mathfrak{h}(F)=\mathfrak{h}^{0}(F) \oplus \mathfrak{h}^{2}(F) \oplus \mathfrak{h}^{4}(F) \oplus \mathfrak{h}^{6}(F) \oplus \mathfrak{h}^{8}(F)$ for the Chow-Künneth decomposition of $F$ obtained in Theorem 3.3. Here $\mathfrak{h}(F)$ is the Chow motive of $F$ and $\mathfrak{h}^{2 i}(F)$ is the Chow motive $\left(F, \pi^{2 i}\right)$. Then the motive of $F \times F$ has a Chow-Künneth decomposition $\bigoplus_{i=0}^{8} \mathfrak{h}^{2 i}(F \times F)$, where $\mathfrak{h}^{2 i}(F \times F):=\bigoplus_{j=0}^{i} \mathfrak{h}^{2 j}(F) \otimes \mathfrak{h}^{2 i-2 j}(F)$. The Bloch-Beilinson conjectures imply that $\mathrm{CH}^{2}(F \times$ $F)_{\text {hom }}=\mathrm{CH}^{2}\left(\mathfrak{h}^{2}(F \times F)\right)=\mathrm{CH}^{2}\left(\mathfrak{h}^{2}(F) \otimes \mathfrak{h}^{0}(F) \oplus \mathfrak{h}^{0}(F) \otimes \mathfrak{h}^{2}(F)\right)=p_{1}^{*} \mathrm{CH}^{2}\left(\mathfrak{h}^{2}(F)\right) \oplus p_{2}^{*} \mathrm{CH}^{2}\left(\mathfrak{h}^{2}(F)\right)=$ $p_{1}^{*} \mathrm{CH}^{2}(F)_{\text {hom }} \oplus p_{2}^{*} \mathrm{CH}^{2}(F)_{\text {hom }}$. Therefore, there exist $\mu$ and $\nu \in \mathrm{CH}^{2}(F)_{\text {hom }}$ such that

$$
E:=p_{1}^{*} \mu+p_{2}^{*} \nu
$$

That $\varepsilon:=\iota_{\Delta}^{*} E=0$ yields $\mu+\nu=0$. On the other hand, if one assumes that ${ }^{t} L=L$ and ${ }^{t}(L+E)=L+E$, then ${ }^{t} E=E$, so that $\mu=\nu$. We conclude that $\mu=\nu=0$ and hence that $E=0$.

Remark 3.5. As will be shown in Sections 14 and 19, if $F$ is the Hilbert scheme of length- 2 subschemes on a K3 surface or the variety of lines on a cubic fourfold, then there is a symmetric cycle $L \in \mathrm{CH}^{2}(F \times$ $F$ ) representing the Beauville-Bogomolov class $\mathfrak{B}$ that satisfies (6), (7), (8) and (9). Moreover, the 
Fourier decomposition induced by such a cycle $L \in \mathrm{CH}^{2}(F \times F)$ satisfies the extra assumption $\mathrm{CH}^{2}(F)_{2}$. $\mathrm{CH}^{2}(F)_{2} \subseteq \mathrm{CH}^{4}(F)_{4}$ of Proposition 3.4 (and in fact $\left.\mathrm{CH}^{2}(F)_{2} \cdot \mathrm{CH}^{2}(F)_{2}=\mathrm{CH}^{4}(F)_{4}\right)$; see Proposition 12.9 and Theorem 20.2 .

\section{First multiplicative Results}

4.1. Intersection with $l$. In this paragraph, we consider a hyperkähler fourfold $F$ endowed with a cycle $L \in \mathrm{CH}^{2}(F \times F)$ representing the Beauville-Bogomolov class $\mathfrak{B}$ that satisfies hypotheses (6), (7), (8) and (9). These hypotheses which are prerequisites to establishing the Fourier decomposition for $F$ are related to the understanding of the intersection of $l$ with 2-cycles. Here, we basically reformulate these hypotheses in the context of the Fourier decomposition.

First, it is straightforward to extract from the statements of Theorems 2.2 and 2.4 the following.

Proposition 4.1. We have

$$
\mathrm{CH}^{4}(F)_{2}=\langle l\rangle \cdot \mathrm{CH}^{2}(F)_{2} \text {. }
$$

Second, (9) can be reformulated as the following.

Proposition 4.2. We have

$$
\mathrm{CH}^{4}(F)_{0}=\langle l\rangle \cdot \mathrm{CH}^{2}(F)_{0} .
$$

More precisely, we have

$$
W_{1}^{2}=\operatorname{ker}\left\{l \cdot: \mathrm{CH}^{2}(F) \rightarrow \mathrm{CH}^{4}(F)\right\} .
$$

Proof. Recall from Theorems $2.2 \& 2.4$ that $\mathrm{CH}^{2}(F)=\mathrm{CH}^{2}(F)_{0} \oplus \mathrm{CH}^{2}(F)_{2}$ with $\mathrm{CH}^{2}(F)_{0}=\langle l\rangle \oplus \Lambda_{2}^{2}$, $\mathrm{CH}^{2}(F)_{2}=\Lambda_{0}^{2}$, and that $l \cdot: \mathrm{CH}^{2}(F)_{2} \rightarrow \mathrm{CH}^{4}(F)$ is injective. We also have $\Lambda_{2}^{2}=W_{1}^{2}$. Thus, because $\mathrm{CH}^{4}(F)_{0}=\left\langle l^{2}\right\rangle$, we are reduced to prove that $l \cdot \Lambda_{2}^{2}=0$. But then, this is (39).

4.2. Intersection of divisors. In this paragraph, we consider a hyperkähler fourfold $F$ which is either the Hilbert scheme of length-2 subschemes on a K3 surface or the variety of lines on a smooth cubic fourfold. It will be shown in Parts 2 and 3 that there exists a cycle $L \in \mathrm{CH}^{2}(F \times F)$ representing the Beauville-Bogomolov class $\mathfrak{B}$ that satisfies hypotheses (6), (7), (8) and (9). Moreover, it will also be the case that $l:=\iota_{\Delta}^{*} L=\frac{5}{6} c_{2}(F)$, where $\iota_{\Delta}: F \rightarrow F \times F$ is the diagonal embedding. The goal here is to prove Theorem 4.6, which sets in the context of the Fourier decomposition the following result.

Theorem 4.3 (Voisin [54]). Let $F$ be either the Hilbert scheme of length-2 subschemes on a K3 surface or the variety of lines on a smooth cubic fourfold. Then any polynomial cohomological relation $\left.P\left(\left[c_{1}\left(L_{j}\right)\right] ;\left[c_{i}(F)\right)\right]\right)=0$ in $\mathrm{H}^{2 k}(F, \mathbb{Q}), L_{j} \in \mathrm{Pic} F$, already holds at the level of Chow groups : $P\left(c_{1}\left(L_{j}\right) ; c_{i}(F)\right)=$ 0 in $\mathrm{CH}^{k}(F)$.

Lemma 4.4. Let $D_{i}, D_{i}^{\prime} \in \mathrm{CH}^{1}(F)$ be divisors on $F$. Then

(i) $D_{1} \cdot D_{2} \in W_{1}^{2} \oplus\langle l\rangle\left(=\mathrm{CH}^{2}(F)_{0}\right)$;

(ii) $\sum_{i=1}^{m} D_{i} \cdot D_{i}^{\prime} \in W_{1}^{2}$ if and only if $\sum_{i=1}^{m} q_{F}\left(\left[D_{i}\right],\left[D_{i}^{\prime}\right]\right)=0$.

Proof. By Proposition $1.3, l \cdot L_{*}\left(D_{1} \cdot D_{2}\right)$ vanishes. Therefore, according to (6), we have

$$
\left(L^{2}\right)_{*}\left(D_{1} \cdot D_{2}\right)=2 D_{1} \cdot D_{2}-\frac{2}{25} L_{*}\left(l \cdot D_{1} \cdot D_{2}\right)+\frac{1}{25}\left(\int_{F}[l] \cup\left[D_{1}\right] \cup\left[D_{2}\right]\right) l .
$$

By Theorem 4.3 and the fact that $l=\frac{5}{6} c_{2}(F), l \cdot D_{1} \cdot D_{2}$ is a multiple of $l^{2}$, so that $L_{*}\left(l \cdot D_{1} \cdot D_{2}\right)=0$. It is also a fact that $\int_{F}[l] \cup\left[D_{1}\right] \cup\left[D_{2}\right]=25 q_{F}\left(\left[D_{1}\right],\left[D_{2}\right]\right)$. Hence

$$
\left(L^{2}\right)_{*}\left(D_{1} \cdot D_{2}\right)=2 D_{1} \cdot D_{2}+q_{F}\left(\left[D_{1}\right],\left[D_{2}\right]\right) l,
$$

which establishes item (ii). Since $\left(L^{2}\right)_{*} l=25 l$ by Theorem 2.2 , we find that

$$
\left(L^{2}-25\right)_{*}\left(L^{2}-2\right)_{*}\left(D_{1} \cdot D_{2}\right)=0,
$$

that is, $D_{1} \cdot D_{2}$ belongs to $\Lambda_{2}^{2} \oplus \Lambda_{25}^{2}$. By Theorem 2.4, $\Lambda_{2}^{2}=W_{1}^{2}$ and $\Lambda_{25}^{2}=\langle l\rangle$, and item (i) is proved. 
Lemma 4.5. Let $D \in \mathrm{CH}^{1}(F)$ be a divisor. Then

(i) $L_{*} D^{3}=3 q_{F}([D]) D$;

(ii) $\left(L^{2}\right)_{*} D^{3}=0$;

(iii) $D^{3}=\frac{3}{25} q_{F}([D]) l \cdot D$.

Proof. (i) $-L_{*} D^{3}$ is a divisor whose cohomology class is

$$
\left[L_{*} D^{3}\right]=\sum_{i=1}^{23}\left(\left[D^{3}\right] \cup e_{i}\right) e_{i}=\sum_{i=1}^{23} 3 q_{F}([D]) q_{F}\left([D], e_{i}\right) e_{i}=3 q_{F}([D])[D] .
$$

Thus $L_{*} D^{3}=3 q_{F}([D]) D$.

(ii) and (iii) - According to (6), together with the above, we have

$$
\left(L^{2}\right)_{*} D^{3}=2 D^{3}-\frac{2}{25} l \cdot L_{*} D^{3}=2 D^{3}-\frac{2 \cdot 3}{25} q_{F}([D]) l \cdot D .
$$

Since by Theorem $2.2 \mathrm{CH}^{3}(F)$ splits as $\Lambda_{0}^{3} \oplus \Lambda_{2}^{3}$ under the action of $L^{2}$, with $\Lambda_{2}^{3}=\mathrm{CH}^{3}(F)_{\text {hom }}$, we get $2\left[D^{3}\right]-\frac{2 \cdot 3}{25} q_{F}([D])[l] \cdot[D]=0$. Theorem 4.3 yields $2 D^{3}-\frac{2 \cdot 3}{25} q_{F}([D]) l \cdot D=0$.

Theorem 4.6. Let $P\left(D_{i}, l\right), D_{i} \in \mathrm{CH}^{1}(F)$, be a polynomial. Then

$$
P\left(D_{i}, l\right) \in \bigoplus_{i} \mathrm{CH}^{i}(F)_{0}
$$

Proof. By Theorem 2.4, we have to show that $P\left(D_{i}, l\right) \in\left\langle l^{2}\right\rangle \oplus W_{\frac{25}{2}}^{3} \oplus\langle l\rangle \oplus W_{1}^{2} \oplus \mathrm{CH}^{1}(F) \oplus\left\langle l^{0}\right\rangle$. Given Theorem 4.3 and Lemma 4.4, we only need to show that $P\left(D_{i}, l\right) \in W_{\frac{25}{2}}^{3}$ for all weighted homogeneous $P$ of degree 3. First we note that the intersection of three divisors can be written as a linear combination of cycles of the form $D^{3}$. Since $l \cdot D$ is proportional to $D^{3}$, we only need to show that $D^{3} \in W_{\frac{25}{2}}^{3}=\Lambda_{0}^{3}$. Observe that by Lemma 4.5 we have

$$
l \cdot L_{*} D^{3}=3 q_{F}([D]) l \cdot D=25 D^{3},
$$

so that the proof follows from (33).

Remark 4.7. By Theorems $2.2 \& 2.4$ and Lemma 4.5, we actually have $\mathrm{CH}^{3}(F)_{0}=\left(\mathrm{CH}^{1}(F)\right)^{\cdot 3}=$ $\langle l\rangle \cdot \mathrm{CH}^{1}(F)$.

Let us end this section with the following lemma, which will be used in Part 2 and Part 3.

Lemma 4.8. Let $\mathrm{V}_{F} \subset \mathrm{CH}^{*}(F)$ be the sub-algebra generated by all divisors and Chern classes of $F$. Then any cohomological polynomial relation $P$ among elements of the sub-algebra of $\mathrm{CH}^{*}(F \times F)$ generated by $p_{1}^{*} \mathrm{~V}_{F}$ and $p_{2}^{*} \mathrm{~V}_{F}$ holds in the Chow ring $\mathrm{CH}^{*}(F \times F)$.

Proof. This is a consequence of Theorem 4.3. We assume that $P$ is a polynomial of bidegree $\left(e_{1}, e_{2}\right)$. We take a basis

$$
\left\{\mathfrak{a}_{1}, \mathfrak{a}_{2}, \ldots, \mathfrak{a}_{m}\right\}
$$

of $\mathrm{V}_{F} \cap \mathrm{CH}^{e_{1}}(F)$. Then we can write

$$
P=\sum_{i=1}^{m} p_{1}^{*} \mathfrak{a}_{i} \cdot p_{2}^{*} P_{i}
$$

where $P_{i}$ are polynomials in $\mathrm{V}_{F}$ of degree $e_{2}$. There exists a basis $\left\{\mathfrak{b}_{1}, \mathfrak{b}_{2}, \ldots \mathfrak{b}_{m}\right\}$ of $\mathrm{V}_{F} \cap \mathrm{CH}^{4-e_{1}}(F)$ such that $\operatorname{deg}\left(\mathfrak{a}_{i} \cdot \mathfrak{b}_{j}\right)=\delta_{i j}$. If $P=0$ in cohomology, then $P_{i}=P_{*} \mathfrak{b}_{i}$ is homologically trivial. It follows from Proposition 4.3 that $P_{i}=0$. Consequently, we get $P=0$ at the Chow group level. 


\section{An APPLICATION TO SYMPLECTIC AUTOMORPHiSMS}

The results of this section will not be used in the rest of this manuscript. The goal here is to show that the Fourier decomposition reduces the question of understanding the action $f^{*}$ of a morphism $f: F \rightarrow F$ on the Chow group of zero-cycles of a hyperkähler variety $F$ to the understanding of the action of $f^{*}$ on certain codimension-two cycles ; see Proposition 5.1. We then use recent results of Voisin [56], Huybrechts [30] and Fu [23] to exemplify this method to the cases where $F$ is the Hilbert scheme of length-2 subschemes on a K3 surface or the variety of lines on a cubic fourfold ; see Proposition 5.2 and Theorem 5.3, respectively. Note that, in both cases, we show that our cycle $L$ representing the Beauville-Bogomolov class $\mathfrak{B}$ is preserved under the action of any automorphism $f: F \rightarrow F$.

Proposition 5.1. Let $F$ be a hyperkähler variety of dimension $2 d$ for which the decomposition

$$
\mathrm{CH}^{2 d}(F)=\bigoplus_{s=0}^{d} \mathrm{CH}^{2 d}(F)_{2 s}, \quad \mathrm{CH}^{2 d}(F)_{2 s}=l^{d-s} \cdot\left(L_{*} \mathrm{CH}^{2 d}(F)\right)^{\cdot s}
$$

in Conjecture 2 holds true. Let $f: F \rightarrow F$ be a morphism such that $f^{*} \sigma=a \sigma$ for all $\sigma \in L_{*} \mathrm{CH}^{2 d}(F)$ and $f^{*} l=b l$, for some $a, b \in \mathbb{Q}$. Then $f^{*}$ acts as multiplication by $a^{s} b^{d-s}$ on $\mathrm{CH}^{2 d}(F)_{2 s}$. In particular, if $a=b=1$ then $f^{*}=\operatorname{Id}$ on $\mathrm{CH}^{2 d}(F)$.

Proof. By assumption, $\mathrm{CH}^{2 d}(F)_{2 s}$ is generated by elements of the form $l^{d-s} \sigma_{1} \cdots \sigma_{s}$ where $\sigma_{i} \in L_{*} \mathrm{CH}^{2 d}(F)$. The compatibility of $f^{*}$ and the intersection product shows that $f^{*}$ acts as multiplication by $a^{s} b^{d-s}$ on such elements.

Note that by Remark 3.5, Theorem 2 and Proposition 4.1, if $F$ is the Hilbert scheme of length-2 subschemes on a $\mathrm{K} 3$ surface or the variety of lines on a cubic fourfold, then $\mathrm{CH}_{0}(F)$ has a decomposition as in Conjecture 2.

An automorphism $f: F \rightarrow F$ of a hyperkähler variety $F$ is said to be symplectic if $f^{*}=$ Id on $\mathrm{H}^{0}\left(F, \Omega_{F}^{2}\right)$. The conjectures of Bloch-Beilinson predict that a symplectic automorphism acts trivially on $\mathrm{CH}_{0}(F)$; see [32]. This was proved to hold for symplectic involutions on K3 surfaces by Voisin [56] and generalized to symplectic automorphisms of finite order on K3 surfaces by Huybrechts [30].

Combined with Proposition 5.1, these results give the following proposition in the case where $F$ is the Hilbert scheme of length-2 subschemes on a K3 surface $S$.

Proposition 5.2. Let $f: S \rightarrow S$ be a symplectic automorphism of finite order of $S$ and $\hat{f}: F \rightarrow F$ the induced symplectic automorphism of $F=S^{[2]}$, then

$$
\hat{f}^{*}=\mathrm{Id}: \mathrm{CH}_{0}(F) \rightarrow \mathrm{CH}_{0}(F) .
$$

Proof. The notations are those of Part 2. Let $x \in S$ be a point and let $S_{x}$ be the surface parameterizing all length-2 subschemes $W \subset S$ such that $x \in W$. Since $f$ is an automorphism, one sees that $x \in W$ if and only if $f^{-1} x \in f^{-1} W$. Hence at the level of Chow groups we have $\hat{f}^{*} S_{x}=S_{f^{*} x}$. As a special case, one has $\hat{f}^{*} S_{\mathfrak{o}}=S_{\mathfrak{o}}$, since $\mathfrak{o}$ is the class of any point on a rational curve of $S$ and $f$ maps a rational curve to a rational curve. Similarly, a length-2 subscheme $W \subset S$ is non-reduced if and only if $f^{-1} W$ is non-reduced. This implies $\hat{f}^{*} \delta=\delta$. Since $f$ preserves the incidence of subschemes of $S$, we have $(\hat{f} \times \hat{f})^{*} I=I$. It follows that $(\hat{f} \times \hat{f})^{*} L=L$, where $L$ is as in (92). Thus we get

$$
\hat{f}^{*} l=\hat{f}^{*}\left(\iota_{\Delta}\right)^{*} L=\left(\iota_{\Delta}\right)^{*}(\hat{f} \times \hat{f})^{*} L=\left(\iota_{\Delta}\right)^{*} L=l .
$$

Now since $L_{*} \mathrm{CH}_{0}(F)$ is generated by cycles of the form $S_{x}-S_{y}$, we see that the condition $\hat{f}^{*}=\operatorname{Id}$ on $L_{*} \mathrm{CH}_{0}(F)$ is implied by $f^{*}=\mathrm{Id}$ on $\mathrm{CH}_{0}(S)_{\text {hom }}$, which was established by Voisin [56] and Huybrechts [30]. We can then invoke Proposition 5.1 with $a=b=1$ to conclude.

In the case where $F$ is the variety of lines on a cubic fourfold, let us mention the following recent result of $\mathrm{L} . \mathrm{Fu}[23]$. 
Theorem $5.3(\mathrm{Fu}[23])$. Let $f: X \rightarrow X$ be an automorphism of the cubic fourfold $X$ such that $f^{*}=\operatorname{Id}$ on $\mathrm{H}^{3,1}(X) \rightarrow \mathrm{H}^{3,1}(X)$ and $\hat{f}: F \rightarrow F$ the induced symplectic automorphism of $F$, then

$$
\hat{f}^{*}=\mathrm{Id}: \mathrm{CH}_{0}(F) \rightarrow \mathrm{CH}_{0}(F) \text {. }
$$

Proof. The notations are those of Part 3. We reproduce, in our setting, the original argument of Fu. First we note that the polarization $g$ on $F$ is represented by the divisor of all lines meeting $H_{1} \cap H_{2}$, where $H_{1}$ and $H_{2}$ are two general hyperplane sections on $X$. Since $f$ maps a line to a line and maps a hyperplane section to a hyperplane section, we have $\hat{f}^{*} g=g$. Note that the class $c$ is represented by all lines contained in some hyperplane section, and hence we get $\hat{f}^{*} c=c$. For a point $t \in F$, with associated line $l_{t} \subset X$, we define $S_{l_{t}}$ to be the surface of all lines meeting the line $l_{t}$. Since $f$ is an automorphism of $X$ that preserves the degrees of subvarieties, we see that $l_{t}$ meets $l_{t^{\prime}}$ if and only if $f^{-1} l_{t}$ meets $f^{-1} l_{t^{\prime}}$. From this we deduce $(\hat{f} \times \hat{f})^{*} I=I$ and $\hat{f}^{*} S_{l_{t}}=S_{f^{*} l_{t}}$, for all $t \in F$. It follows that $(\hat{f} \times \hat{f})^{*} L=L$, where $L$ is as in (107). Thus we get

$$
\hat{f}^{*} l=\hat{f}^{*}\left(\iota_{\Delta}\right)^{*} L=\left(\iota_{\Delta}\right)^{*}(\hat{f} \times \hat{f})^{*} L=\left(\iota_{\Delta}\right)^{*} L=l .
$$

Now since $L_{*} \mathrm{CH}_{0}(F)$ is generated by cycles of the form $S_{l_{t_{1}}}-S_{l_{t_{2}}}$, we see that the condition $\hat{f}^{*}=\operatorname{Id}$ on $L_{*} \mathrm{CH}^{4}(F)$ is reduced to

$$
l_{t_{1}}-l_{t_{2}}=f^{*} l_{t_{1}}-f^{*} l_{t_{2}}, \quad \text { in } \mathrm{CH}^{3}(X) .
$$

The above equality is implied by $f^{*}=\mathrm{Id}$ on $\mathrm{CH}^{3}(X)_{\text {hom }}$, which was established by $\mathrm{Fu}$ [23]. Hence we can conclude by Proposition 5.1 with $a=b=1$.

\section{ON THE BIRATIONAL INVARIANCE OF THE FOURIER DECOMPOSITION}

Ulrike Greiner [26] has recently proved the following theorem.

Theorem 6.1 (Greiner [26]). Let $F$ and $F^{\prime}$ be birational hyperkähler varieties of $\mathrm{K}^{[2]}$-type. Then there exists a correspondence $\gamma \in \mathrm{CH}^{4}\left(F \times F^{\prime}\right)$ such that $\gamma_{*}: \mathrm{CH}^{*}(F) \rightarrow \mathrm{CH}^{*}\left(F^{\prime}\right)$ is an isomorphism of graded rings.

The main result of this section is Theorem 6.5, which roughly states that if $F$ and $F^{\prime}$ are birational hyperkähler varieties of $\mathrm{K}^{[2]}$-type and if the Chow ring of $F$ has a Fourier decomposition with respect to a cycle $L \in \mathrm{CH}^{2}(F \times F)$ representing the Beauville-Bogomolov class $\mathfrak{B}$, then the Chow ring of $F^{\prime}$ also has a Fourier decomposition with respect to a cycle $L^{\prime} \in \mathrm{CH}^{2}\left(F^{\prime} \times F^{\prime}\right)$ representing the BeauvilleBogomolov class $\mathfrak{B}^{\prime} \in \mathrm{H}^{4}\left(F^{\prime} \times F^{\prime}, \mathbb{Q}\right)$. Thus Theorem 3 also holds for any hyperkähler variety birational to the Hilbert scheme of length-2 subschemes on a K3 surface, or to the variety of lines on a very general cubic fourfold. Theorem 6.5 is a generalization of Theorem 6.1 and the method of proof follows closely that of Greiner.

The following result is due to Huybrechts [28, Theorem 3.4 \& Theorem 4.7] in the non-algebraic setting. As noted in [26], the proof adapts to the algebraic setting at the condition that the two varieties $F$ and $F^{\prime}$ are connected by Mukai flops, which is the case for hyperkähler fourfolds of $\mathrm{K} 3^{[2]}$-type by [58].

Theorem 6.2 (Huybrechts [28]). Let $F$ and $F^{\prime}$ be birational hyperkähler fourfolds of $\mathrm{K}^{[2]}{ }^{[2]}$ type. Then there exist algebraic varieties $\mathscr{F}$ and $\mathscr{F}^{\prime}$ smooth and projective over a smooth quasi-projective curve $T$, and a closed point $0 \in T$ such that

(i) $\mathscr{F}_{0}=F$ and $\mathscr{F}_{0}^{\prime}=F^{\prime}$;

(ii) there is an isomorphism $\Psi: \mathscr{F}_{T \backslash\{0\}} \stackrel{\simeq}{\longrightarrow} \mathscr{F}_{T \backslash\{0\}}^{\prime}$ over $T \backslash\{0\}$.

In the rest of this section, we are in the situation of Theorem 6.2. Let $\eta \rightarrow T$ be the generic point of $T$ and let $\gamma_{\eta} \in \mathrm{CH}^{4}\left(\mathscr{F}_{\eta} \times_{\eta} \mathscr{F}_{\eta}^{\prime}\right)$ be the class of the restriction to the generic fiber of the graph of $\Psi$. The isomorphism $\Psi$ restricts to an isomorphism $\psi: \mathscr{F}_{\eta} \rightarrow \mathscr{F}_{\eta}^{\prime}$, and $\gamma_{\eta}$ is nothing but the class of the graph of $\psi$. In particular, we have $\gamma_{\eta} \circ{ }^{t} \gamma_{\eta}=\Delta_{\mathscr{F}_{\eta}^{\prime}}$ and ${ }^{t} \gamma_{\eta} \circ \gamma_{\eta}=\Delta_{\mathscr{F}_{\eta}}$, where ${ }^{t} \gamma_{\eta}$ denotes the transpose of $\gamma_{\eta}$. Let us write $s_{0}$ for Fulton's specialization map on Chow groups of varieties which are smooth over $T$; see $[25, \S 20.3]$. If $\mathscr{X} \rightarrow T$ is a smooth morphism, then the specialization map $s_{0}: \mathrm{CH}^{*}\left(\mathscr{X}_{\eta}\right) \rightarrow \mathrm{CH}^{*}\left(\mathscr{X}_{0}\right)$ 
sends a cycle $\alpha$ on the generic fiber $\mathscr{X}_{\eta}$ of $\mathscr{X} \rightarrow T$ to the restriction to the special fiber $\mathscr{X}_{0}$ of a closure of $\alpha$ to $\mathrm{CH}^{*}(\mathscr{X})$. This map commutes with intersection product, flat pull-backs and proper push-forwards. In particular, if we are dealing with varieties which are smooth and projective over $T$, then $s_{0}$ commutes with composition of correspondences. We set

$$
\gamma:=s_{0}\left(\gamma_{\eta}\right) \in \mathrm{CH}^{4}\left(F \times F^{\prime}\right) .
$$

U. Greiner showed [26, Theorem 2.6] that $\gamma_{*}: \mathrm{CH}^{*}(F) \rightarrow \mathrm{CH}^{*}\left(F^{\prime}\right)$ is an isomorphism of graded rings with inverse given by $\gamma^{*}$. Let us, for clarity, reproduce Greiner's proof. Specializing the identities $\gamma_{\eta} \circ{ }^{t} \gamma_{\eta}=\Delta_{\mathscr{F}_{\eta}^{\prime}}$ and ${ }^{t} \gamma_{\eta} \circ \gamma_{\eta}=\Delta_{\mathscr{F}_{\eta}}$ immediately gives $\gamma \circ{ }^{t} \gamma=\Delta_{F^{\prime}}$ and ${ }^{t} \gamma \circ \gamma=\Delta_{F}$, so that $\gamma_{*}: \mathrm{CH}^{*}(F) \rightarrow \mathrm{CH}^{*}\left(F^{\prime}\right)$ is a group isomorphism. Given a smooth projective variety $X$, the small diagonal $\Delta_{123}^{X} \in \mathrm{CH}^{*}(X \times X \times X)$ is the class of the graph of the diagonal embedding $\iota_{\Delta}: X \rightarrow X \times X$ that we see as a correspondence from $X \times X$ to $X$. Thus, given cycles $\alpha, \beta \in \mathrm{CH}^{*}(X)$, we have

$$
\alpha \cdot \beta=\iota_{\Delta}^{*}(\alpha \times \beta)=\Delta_{123, *}^{X}(\alpha \times \beta) .
$$

In order to prove that $\gamma_{*}$ is a ring homomorphism, it is therefore enough to check that $\gamma \circ \Delta_{123}^{F}=$ $\Delta_{123}^{F^{\prime}} \circ(\gamma \times \gamma)$, or equivalently that $\gamma \circ \Delta_{123}^{F} \circ\left({ }^{t} \gamma \times{ }^{t} \gamma\right)=\Delta_{123}^{F^{\prime}}$. By specialization, it is enough to prove that $\gamma_{\eta} \circ \Delta_{123}^{\mathscr{F}_{\eta}} \circ\left({ }^{t} \gamma_{\eta} \times{ }^{t} \gamma_{\eta}\right)=\Delta_{123}^{\mathscr{F}_{\eta}^{\prime}}$. But then, by [51, Lemma 3.3], we have

$$
(\beta \times \alpha)_{*} Z=\alpha \circ Z \circ{ }^{t} \beta \in \mathrm{CH}^{*}\left(X^{\prime} \times Y^{\prime}\right),
$$

for all $X, X^{\prime}, Y$ and $Y^{\prime}$ smooth projective varieties and all correspondences $Z \in \mathrm{CH}^{*}(X \times Y), \alpha \in$ $\mathrm{CH}^{*}\left(Y \times Y^{\prime}\right)$ and $\beta \in \mathrm{CH}^{*}\left(X \times X^{\prime}\right)$. Hence, by (40), we have $\gamma_{\eta} \circ \Delta_{123}^{\mathscr{F}_{\eta}} \circ\left({ }^{t} \gamma_{\eta} \times{ }^{t} \gamma_{\eta}\right)=\left(\gamma_{\eta} \times \gamma_{\eta} \times \gamma_{\eta}\right)_{*} \Delta_{123}^{\mathscr{F}_{\eta}}$, which clearly is equal to $\Delta_{123}^{\mathscr{F}_{\eta}^{\prime}}$.

Note that replacing $\Psi$ by $\Psi \times \ldots \times \Psi$, the above also shows that $(\gamma \times \ldots \times \gamma)_{*}: \mathrm{CH}^{*}(F \times \ldots \times F) \rightarrow$ $\mathrm{CH}^{*}\left(F^{\prime} \times \ldots \times F^{\prime}\right)$ is an isomorphism of graded rings with inverse $(\gamma \times \ldots \times \gamma)^{*}$. The compatibility of the Fourier decomposition with the intersection product can be expressed purely in terms of the intersection theoretical properties of $L$ :

Lemma 6.3. Let $L \in \mathrm{CH}^{*}(F \times F)$ be a correspondence and let $L^{\prime}:=(\gamma \times \gamma)_{*} L$. Then, for all integers $p, q, r \geq 0$, we have

$$
\begin{aligned}
\gamma \circ L^{r} \circ \Delta_{123}^{F} \circ\left(p_{13}^{*} L^{p} \cdot p_{24}^{*} L^{q}\right) & =\left(L^{\prime}\right)^{r} \circ \Delta_{123}^{F^{\prime}} \circ\left(p_{13}^{* *}\left(L^{\prime}\right)^{p} \cdot p_{24}^{\prime *}\left(L^{\prime}\right)^{q}\right) \circ(\gamma \times \gamma), \\
\gamma \circ L^{r} \circ\left(p_{i}^{*} \iota_{\Delta}^{*} L^{p} \cdot L^{q}\right) & =\left(L^{\prime}\right)^{r} \circ\left(p_{i}^{\prime *} \iota_{\Delta}^{*}\left(L^{\prime}\right)^{p} \cdot\left(L^{\prime}\right)^{q}\right) \circ \gamma, \\
\gamma \circ L^{r} \circ\left(p_{i}^{*} \iota_{\Delta}^{*} L^{p} \cdot p_{j}^{*} \iota_{\Delta}^{*} L^{q}\right) & =\left(L^{\prime}\right)^{r} \circ\left(p_{i}^{\prime *} \iota_{\Delta}^{*}\left(L^{\prime}\right)^{p} \cdot p_{j}^{\prime *} \iota_{\Delta}^{*}\left(L^{\prime}\right)^{q}\right) \circ \gamma,
\end{aligned}
$$

where $p_{i}: F \times F \rightarrow F$ is the projection on the $i^{\text {th }}$ factor, $p_{i j}: F \times F \times F \times F \rightarrow F \times F$ is the projection on the product of the $i^{\text {th }}$ and $j^{\text {th }}$ factors, and similarly for $F^{\prime}$.

Proof. If $Z \in \mathrm{CH}^{*}(X \times Y)$ and $\alpha \in \mathrm{CH}^{*}(X)$, then we have the following formula

$$
Z_{*} \alpha=Z \circ \alpha \in \mathrm{CH}^{*}(Y),
$$

where on the right-hand side of the equality $\alpha$ is seen as a correspondence from a point to $X$. For instance, we have $p_{i}^{*} \iota_{\Delta}^{*} L^{p}={ }^{t} \Gamma_{p_{i}} \circ \Delta_{123}^{F} \circ L^{p}$, where on the right-hand side $L^{p}$ is seen as a correspondence from a point to $F \times F$. Thus the main point consists in checking that $\gamma$ commutes with $L$ and its powers, with projections and with diagonals. Precisely, writing $\Gamma_{f} \in \mathrm{CH}_{\operatorname{dim} X}(X \times Y)$ for the class of the graph of a morphism $f: X \rightarrow Y$, we claim that

$$
\gamma \circ L^{r}=L^{\prime r} \circ \gamma, \quad \gamma \circ \Delta_{123}^{F}=\Delta_{123}^{F^{\prime}} \circ(\gamma \times \gamma) \quad \text { and } \quad \gamma \circ \Gamma_{p_{i}}=\Gamma_{p_{i}^{\prime}} \circ(\gamma \times \gamma) .
$$

The following identities have already been established : $\gamma \circ{ }^{t} \gamma=\Delta_{F^{\prime}},{ }^{t} \gamma \circ \gamma=\Delta_{F}$ and $\gamma \circ \Delta_{123}^{F} \circ$ $\left({ }^{t} \gamma \times{ }^{t} \gamma\right)=\Delta_{123}^{F^{\prime}}$. Recall that $(\gamma \times \ldots \times \gamma)_{*}$ is multiplicative. Thus $(\gamma \times \gamma)_{*}\left(L^{r}\right)=\left((\gamma \times \gamma)_{*} L\right)^{r}=\left(L^{\prime}\right)^{r}$. By (40), we get $\gamma \circ L^{r} \circ{ }^{t} \gamma=L^{\prime r}$ and hence, by composing with $\gamma$ on the right, $\gamma \circ L^{r}=L^{\prime r} \circ \gamma$. Therefore it remains only to check that for $p_{i_{1}, \ldots, i_{s}}: F^{n} \rightarrow F^{s}$ the projection on the $\left(i_{1}, \ldots, i_{s}\right)$-factor, we have $\gamma^{\times s} \circ \Gamma_{p_{i_{1}, \ldots, i_{s}}}=\Gamma_{p_{i_{1}, \ldots, i_{s}}^{\prime}} \circ \gamma^{\times n}$, where $\Gamma_{p_{i_{1}, \ldots, i_{s}}}$ is the class of the graph of $p_{i_{1}, \ldots, i_{s}}$ and where $p_{i_{1}, \ldots, i_{s}}^{\prime}: F^{\prime n} \rightarrow F^{\prime s}$ is the projection on the $\left(i_{1}, \ldots, i_{s}\right)$-factor. But then, this follows immediately from $\gamma^{\times n} \circ\left({ }^{t} \gamma\right)^{\times n}=\Delta_{F^{n}}$ and from the identity $\gamma^{\times s} \circ \Gamma_{p_{i_{1}, \ldots, i_{s}}} \circ\left({ }^{t} \gamma\right)^{\times n}=\left(\gamma^{\times(n+s)}\right)_{*} \Gamma_{p_{i_{1}, \ldots, i_{s}}}=\Gamma_{p_{i_{1}, \ldots, i_{s}}^{\prime}}$. 
MINGMIN SHEN AND CHARLES VIAL

Lemma 6.4. Let $F$ be a hyperkähler fourfold of $\mathrm{K}^{[2]}$-type. Assume that there is a cycle $L \in \mathrm{CH}^{2}(F \times F)$ with cohomology class $\mathfrak{B}$ that satisfies $(6),(7),(8)$ and $(9)$. Let $(i, s)$ and $(j, r)$ be pairs of non-negative integers and set $p:=i-s$ and $q:=j-r$. The inclusion $\mathrm{CH}^{i}(F)_{2 s} \cdot \mathrm{CH}^{j}(F)_{2 r} \subseteq \mathrm{CH}^{i+j}(F)_{2 r+2 s}$ is equivalent to having

(41) $\left(L^{t}\right)_{*}\left(\left(L^{p}\right)_{*} \sigma \cdot\left(L^{q}\right)_{*} \tau\right)=0$, for all $t \neq 4-p-q$, all $\sigma \in \mathrm{CH}^{4-i+2 s}(F)$ and all $\tau \in \mathrm{CH}^{4-j+2 r}(F)$.

Proof. It follows immediately from Theorem 2.4 that

$$
\mathrm{CH}^{i}(F)_{2 s}=\left(L^{i-s}\right)_{*} \mathrm{CH}^{4-i+2 s}(F) \text { for all } i, s
$$

and that

$$
\mathrm{CH}^{i}(F)_{2 s}=\bigcap_{s^{\prime} \neq 4-i+s} \operatorname{ker}\left\{\left(L^{s^{\prime}}\right)_{*}: \mathrm{CH}^{i}(F) \rightarrow \mathrm{CH}^{i-4+2 s^{\prime}}(F)\right\} \text { for all } i, s .
$$

This proves the lemma.

Theorem 6.5. Let $F$ and $F^{\prime}$ be two birational hyperkähler fourfolds of $\mathrm{K}^{[2]}$-type. Assume that there is a cycle $L \in \mathrm{CH}^{2}(F \times F)$ representing the Beauville-Bogomolov class $\mathfrak{B}$ that satisfies (6), (7), (8), or (9). Then the cycle $L^{\prime}:=(\gamma \times \gamma){ }_{*} L \in \mathrm{CH}^{2}\left(F^{\prime} \times F^{\prime}\right)$ represents the Beauville-Bogomolov class $\mathfrak{B}^{\prime}$ on $F^{\prime}$, and satisfies (6), (7), (8), or (9), respectively.

Assume that $L$ satisfies all four conditions (6), (7), (8) and (9) and that the associated Fourier decomposition of Theorem 2.4 satisfies $\mathrm{CH}^{i}(F)_{2 s} \cdot \mathrm{CH}^{j}(F)_{2 r} \subseteq \mathrm{CH}^{i+j}(F)_{2 r+2 s}$ for some $(i, s)$ and $(j, r)$. Then the Fourier decomposition associated to $L^{\prime}$ satisfies $\mathrm{CH}^{i}\left(F^{\prime}\right)_{2 s} \cdot \mathrm{CH}^{j}\left(F^{\prime}\right)_{2 r} \subseteq \mathrm{CH}^{i+j}\left(F^{\prime}\right)_{2 r+2 s}$

Proof. The correspondence $\gamma \in \mathrm{CH}^{4}\left(F \times F^{\prime}\right)$ respects the Beauville-Bogomolov form by Huybrechts [29, Corollary 2.7], so that $\left[L^{\prime}\right]=(\gamma \times \gamma)_{*} \mathfrak{B}=\mathfrak{B}^{\prime}$. If $L$ satisfies (6), then Lemma 6.3 immediately shows that $L^{\prime}=\gamma \circ L \circ{ }^{t} \gamma$ also satisfies (6). As a consequence of Lemma 6.3, we have for all $\sigma^{\prime}, \tau^{\prime} \in \mathrm{CH}^{*}(F)$ and all non-negative integers $p, q, t$,

$$
\left(L^{\prime t}\right)_{*}\left(\left(L^{\prime p}\right)_{*} \sigma^{\prime} \cdot\left(L^{\prime q}\right)_{*} \tau^{\prime}\right)=\gamma_{*}\left(L^{t}\right)_{*}\left(\left(L^{p}\right)_{*} \sigma \cdot\left(L^{q}\right)_{*} \tau\right),
$$

where we have set $\sigma:=\gamma^{*} \sigma^{\prime}$ and $\tau:=\gamma^{*} \tau^{\prime}$. Thus it is clear that if $L$ satisfies one of (7), (8) or (9), then so does $L^{\prime}$.

Assume now that $L$ satisfies all four conditions (6), (7), (8) and (9). Then (44), together with Lemma 6.4, yields that $\mathrm{CH}^{i}(F)_{2 s} \cdot \mathrm{CH}^{j}(F)_{2 r} \subseteq \mathrm{CH}^{i+j}(F)_{2 r+2 s}$ if and only if $\mathrm{CH}^{i}\left(F^{\prime}\right)_{2 s} \cdot \mathrm{CH}^{j}\left(F^{\prime}\right)_{2 r} \subseteq$ $\mathrm{CH}^{i+j}\left(F^{\prime}\right)_{2 r+2 s}$.

Remark 6.6. Let $F$ and $F^{\prime}$ be birational hyperkähler fourfolds of $\mathrm{K}^{[2]}$-type. Assume that $F$ satisfies Conjecture 3. Then the same arguments as the ones above combined with the fact [26, Lemma 3.4] that $\gamma_{*} c_{2}(F)=c_{2}\left(F^{\prime}\right)$ show that $F^{\prime}$ also satisfies Conjecture 3 with $L^{\prime}:=(\gamma \times \gamma)_{*} L$.

\section{An alternate approach to the Fourier Decomposition on the Chow Ring of abelian VARIETIES}

We wish to give new insight in the theory of algebraic cycles on abelian varieties by explaining how Beauville's decomposition theorem [8] on the Chow ring of abelian varieties can be derived directly from a recent powerful theorem of Peter O'Sullivan [43]. This approach is in the same spirit as the proof of Theorem 5, which will be given at the end of Section 8. It is also somewhat closer to our approach for proving the Fourier decomposition for certain hyperkähler fourfolds of $\mathrm{K} 3^{[2]}$-type. At the end of the section, we further explain how O'Sullivan's theorem easily implies finite-dimensionality [33, 42] for the Chow motive of an abelian variety.

We insist that the sole purpose of this section is to illustrate the importance of O'Sullivan's theorem, and to a lesser extent to make manifest how powerful a similar result for hyperkähler varieties ( $c f$. Conjecture 3) would be. In particular, this section is logically ill for two reasons. First, in order to obtain his final result, O'Sullivan establishes Beauville's decomposition theorem from his own machinery (independent of Beauville's) as an intermediate result [43, Theorem 5.2.5]. Second, O'Sullivan uses in a crucial way that the Chow motive of an abelian variety is finite-dimensional [33]. 
Let $A$ be an abelian variety over a field $k$. O'Sullivan introduces the following definition. Consider a cycle $\alpha \in \mathrm{CH}^{*}(A)$. For each integer $m \geq 0$, denote by $V_{m}(\alpha)$ the $\mathbb{Q}$-vector sub-space of $\mathrm{CH}^{*}\left(A^{m}\right)$ generated by elements of the form $p_{*}\left(p_{1}^{*} \alpha^{r_{1}} \cdot p_{2}^{*} \alpha^{r_{2}} \cdot \ldots \cdot p_{n}^{*} \alpha^{r_{n}}\right)$, where $n \leq m$, the $r_{j}$ are integers $\geq 0$, $p_{i}: A^{n} \rightarrow A$ are the projections on the $i^{\text {th }}$ factor, and $p: A^{n} \rightarrow A^{m}$ is a closed immersion with each component $A^{n} \rightarrow A$ either a projection or the composite of a projection with [-1] :A $\rightarrow A$. Then $\alpha$ is called symmetrically distinguished if for every $m$ the restriction of the quotient map $\mathrm{CH}^{*}\left(A^{m}\right) \rightarrow$ $\mathrm{CH}^{*}\left(A^{m}\right) / \sim_{\text {num }}$, where $\sim_{\text {num }}$ is numerical equivalence of cycles, to $V_{m}(\alpha)$ is injective.

Here is the main result of [43].

Theorem 7.1 (O'Sullivan). The symmetrically distinguished cycles in $\mathrm{CH}^{*}(A)$ form a $\mathbb{Q}$-sub-algebra which maps isomorphically to $\mathrm{CH}^{*}(A) / \sim_{\text {num }}$ under the projection $\mathrm{CH}^{*}\left(A^{m}\right) \rightarrow \mathrm{CH}^{*}\left(A^{m}\right) / \sim_{\text {num }}$. Moreover, symmetrically distinguished cycles are stable under push-forward and pull-back of homomorphisms of abelian varieties.

In particular, $[A] \in \mathrm{CH}^{0}(A)$ is symmetrically distinguished and consequently the graphs of homomorphisms $f: A \rightarrow A^{\prime}$ of abelian varieties are symmetrically distinguished. Indeed, we may write $\Gamma_{f}=\left(\operatorname{id}_{A} \times f\right)_{*}[A] \in \mathrm{CH}^{*}\left(A \times A^{\prime}\right)$. According to Theorem 7.1, another class of symmetrically distinguished cycles is given by symmetric divisors on $A$, i.e., divisors $D \in \mathrm{CH}^{1}(A)$ such that $[-1]^{*} D=D$. A direct consequence of Theorem 7.1 is thus the following analogue of Theorem 4.3 in the case of abelian varieties.

Corollary 7.2. The sub-algebra of $\mathrm{CH}^{*}(A)$ generated by symmetric divisors injects into cohomology via the cycle class map.

Note that Corollary 7.2 has been recently obtained by different methods by Ancona [4] and Moonen [36].

Denote $\hat{A}$ the dual abelian variety of $A$. Let $L$ be the Poincaré bundle on $A \times \hat{A}$ seen as an element of $\mathrm{CH}^{1}(A \times \hat{A})$. We write $\hat{L}$ for the transpose of $L ; \hat{L}$ is the Poincaré bundle on $\hat{A} \times A$.

Proposition 7.3. Write $\pi_{\mathrm{hom}}^{i} \in \mathrm{H}^{2 d}(A \times A, \mathbb{Q})$ for the Künneth projector on $\mathrm{H}^{i}(A, \mathbb{Q})$. The Poincaré bundle $L$ satisfies the following cohomological relations:

$$
[\hat{L}]^{j} \circ[L]^{2 d-i}= \begin{cases}0 & \text { if } j \neq i \\ (-1)^{i} i !(2 d-i) ! \pi_{\text {hom }}^{i} & \text { if } j=i .\end{cases}
$$

Proof. The following proof is inspired from [7, Proposition 1]. Let us write $H:=\mathrm{H}^{1}(A, \mathbb{Q})$ and recall that $\mathrm{H}^{k}(A, \mathbb{Q})=\bigwedge^{k} \mathrm{H}^{1}(A, \mathbb{Q})=\bigwedge^{k} H$. Let $e_{1}, \ldots, e_{2 d}$ be a basis of $H$ and let $e_{1}^{\vee}, \ldots, e_{2 d}^{\vee}$ be the dual basis. We then have

$$
[L]=\sum_{k=1}^{2 d} e_{k} \otimes e_{k}^{\vee} \in \mathrm{H}^{2}(A \times \hat{A}, \mathbb{Q}) .
$$

As in the proof of $\left[7\right.$, Proposition 1], we write, for $K=\left\{k_{1}, \ldots, k_{p}\right\}$ a sub-set of $\{1, \ldots, 2 d\}$ with $k_{1}<\ldots<k_{p}, e_{K}:=e_{k_{1}} \wedge \ldots \wedge e_{k_{p}}$ and $e_{K}^{\vee}:=e_{k_{1}}^{\vee} \wedge \ldots \wedge e_{k_{p}}^{\vee}$. A simple calculation yields

$$
[L]^{p}=p ! \sum e_{K} \otimes e_{K}^{\vee}
$$

where the sum runs through all sub-sets $K \subseteq\{1, \ldots, 2 d\}$ of cardinality $|K|=p$. If $J$ is another sub-set of $\{1, \ldots, 2 d\}$, then $\left(e_{K} \otimes e_{K}^{\vee}\right)_{*} e_{J}=0$ unless $J=K^{c}:=\{1, \ldots, 2 d\} \backslash K$ in which case $\left(e_{K} \otimes e_{K}^{\vee}\right)_{*} e_{K^{c}}=$ $\varepsilon(K) e_{K}^{\vee}$, where $\varepsilon(K)= \pm 1$ is such that $e_{K} \wedge e_{K^{c}}=\varepsilon(K) e_{1} \wedge \ldots \wedge e_{2 d}$. Noting that $\varepsilon\left(K^{c}\right) \varepsilon(K)=(-1)^{|K|}$, we get for $I, J$ and $K$ sub-sets of $\{1, \ldots, 2 d\}$

$$
\left(e_{I}^{\vee} \otimes e_{I}\right)_{*}\left(e_{K} \otimes e_{K}^{\vee}\right)_{*} e_{J}= \begin{cases}(-1)^{|K|} e_{K^{c}} & \text { if } I=J=K^{c} \\ 0 & \text { otherwise. }\end{cases}
$$

We therefore see that, if $i \neq j$, then $\left([\hat{L}]^{j} \circ[L]^{2 d-i}\right)_{*}$ acts trivially on $e_{K}^{\vee}$ for all sub-sets $K \subseteq\{1, \ldots, 2 d\}$, i.e., that $[\hat{L}]^{j} \circ[L]^{2 d-i}=0$ for $i \neq j$. On the other hand, if $i=j$ and if $J$ is a sub-set of $\{1, \ldots, 2 d\}$, then $\left([\hat{L}]^{i} \circ[L]^{2 d-i}\right)_{*} e_{J}=0$ unless if $|J|=i$ in which case $\left([\hat{L}]^{i} \circ[L]^{2 d-i}\right)_{*} e_{J}=i !(2 d-i) !\left(e_{J}^{\vee} \otimes e_{J}\right)_{*}\left(e_{J^{c}} \otimes\right.$ $\left.e_{J^{c}}^{\vee}\right)_{*} e_{J}=(-1)^{i} i !(2 d-i) ! e_{J}$. Thus $[\hat{L}]^{j} \circ[L]^{2 d-i}$ acts by multiplication by $(-1)^{i} i !(2 d-i)$ ! on $\mathrm{H}^{i}(A, \mathbb{Q})=$ $\bigwedge^{i} H$, and as zero on $\mathrm{H}^{j}(A, \mathbb{Q})=\bigwedge^{j} H$ if $j \neq i$. 
By the theorem of the square, the Poincaré bundle $L$ is symmetric. Theorem 7.1 then implies that the relations of Proposition 7.3 hold modulo rational equivalence. More precisely, $\hat{L}^{j} \circ L^{2 d-i}=0$ if $j \neq i$ and if we define

$$
\pi^{i}:=\frac{(-1)^{i}}{i !(2 d-i) !} \hat{L}^{i} \circ L^{2 d-i} \text { for all } 0 \leq i \leq 2 d,
$$

then $\left\{\pi^{i}: 0 \leq i \leq 2 d\right\}$ is a Chow-Künneth decomposition of the diagonal $\Delta_{A} \in \mathrm{CH}^{d}(A \times A)$ that consists of symmetrically distinguished cycles. Denote $\mathcal{F}:=e^{L}$ the Fourier transform, it follows that

$$
\mathrm{CH}^{i}(A)=\bigoplus_{s=i-d}^{i} \mathrm{CH}^{i}(A)_{s}, \text { with } \mathrm{CH}^{i}(A)_{s}:=\left(\pi^{2 i-s}\right)^{*} \mathrm{CH}^{i}(A)=\left\{\sigma \in \mathrm{CH}^{i}(A): \mathcal{F}(\sigma) \in \mathrm{CH}^{d-i+s}(\hat{A})\right\} .
$$

Let $\Gamma_{[n]} \in \mathrm{CH}^{d}(A \times A)$ be the class of the graph of the multiplication-by- $n$ map $[n]: A \rightarrow A$. Since $[n]^{*}$ acts by multiplication by $n^{j}$ on $\mathrm{H}^{j}(A, \mathbb{Q})$ and since $\Gamma_{[n]}$ is symmetrically distinguished, Theorem 7.1 yields

and therefore

$$
{ }^{t} \Gamma_{[n]} \circ \pi^{j}=n^{j} \pi^{j}
$$

$$
\mathrm{CH}^{i}(A)_{s}=\left\{\sigma \in \mathrm{CH}^{i}(A):[n]^{*} \sigma=n^{2 i-s} \sigma\right\} .
$$

As a straightforward consequence, we get the multiplicative property of the Fourier decomposition : $\mathrm{CH}^{i}(A)_{s} \cdot \mathrm{CH}^{j}(A)_{r} \subseteq \mathrm{CH}^{i+j}(A)_{r+s}$. Let us however give a proof that does not use the multiplicationby- $n$ map. This will be used in the next section to establish that the Chow-Künneth decomposition $\left\{\pi^{i}: 0 \leq i \leq 2 d\right\}$ is in fact multiplicative; see Example 8.3. As in the proof of Lemma 6.4, the Fourier decomposition may be characterized by

$$
\mathrm{CH}^{i}(A)_{s}=\left(\hat{L}^{2 i-s}\right)_{*} \mathrm{CH}^{d-i+s}(\hat{A})=\bigcap_{s^{\prime} \neq 2 d-2 i+s} \operatorname{ker}\left\{\left(L^{s^{\prime}}\right)_{*}: \mathrm{CH}^{i}(A) \rightarrow \mathrm{CH}^{i-d+s^{\prime}}(\hat{A})\right\} .
$$

Therefore, $\mathrm{CH}^{i}(A)_{s} \cdot \mathrm{CH}^{j}(A)_{r} \subseteq \mathrm{CH}^{i+j}(A)_{r+s}$ holds for all non-negative integers $i, j, r$ and $s$ if and only if $\left(L^{t}\right)_{*}\left(\left(\hat{L}^{p}\right)_{*} \sigma \cdot\left(\hat{L}^{q}\right)_{*} \tau\right)=0$ for all $t+p+q \neq 2 d$ and all $\sigma, \tau \in \mathrm{CH}^{*}(\hat{A})$. By Theorem 7.1 , it is enough to check the following cohomological formula :

$$
[L]^{t} \circ\left[\Delta_{123}^{A}\right] \circ\left(p_{13}^{*}[\hat{L}]^{p} \cdot p_{24}^{*}[\hat{L}]^{q}\right)=0 \text { for all } t+p+q \neq 2 d,
$$

where $\left[\Delta_{123}^{A}\right]$ is the class of the graph of the diagonal embedding $\iota_{\Delta}: A \rightarrow A \times A$ seen as a correspondence from $A \times A$ to $A$, and where $p_{i j}: A \times A \times A \times A \rightarrow A \times A$ is the projection on the $i^{\text {th }}$ and $j^{\text {th }}$ factor. But then this is straightforward as $[L]_{*}^{p}: \mathrm{H}^{*}(A, \mathbb{Q}) \rightarrow \mathrm{H}^{*}(\hat{A}, \mathbb{Q})$ acts as zero on $\mathrm{H}^{q}(A, \mathbb{Q})$ for $q+p \neq 2 d$.

Finally, we highlight the fact that O'Sullivan's Theorem 7.1 encompasses Kimura-O'Sullivan's finitedimensionality [33] for abelian varieties. Indeed, the cycles

$$
p_{S^{n}}:=\frac{1}{n !} \sum_{\sigma \in \mathfrak{S}_{n}} \Gamma_{\sigma} \quad \text { and } \quad p_{\Lambda^{n}}:=\frac{1}{n !} \sum_{\sigma \in \mathfrak{S}_{n}} \epsilon(\sigma) \Gamma_{\sigma} \quad \text { in } \mathrm{CH}^{d n}\left(A^{n} \times A^{n}\right)
$$

are clearly symmetrically distinguished. Here $\mathfrak{S}_{n}$ is the symmetric group on $n$ elements, $\epsilon$ is the signature homomorphism and $\Gamma_{\sigma}$ is the graph of the action of $\sigma$ on $X^{n}$ that permutes the factors. Having in mind the symmetrically distinguished Chow-Künneth projectors $\pi^{i}$ of (45), we consider the projectors on the even and odd degree part of the cohomology :

$$
\pi_{+}:=\sum_{i} \pi^{2 i} \quad \text { and } \quad \pi_{-}:=\sum_{i} \pi^{2 i+1} \quad \text { in } \mathrm{CH}^{d}(A \times A)
$$

For a cycle $\alpha \in \mathrm{CH}^{d}(A \times A)$, we denote $\alpha^{\otimes n}$ the cycle $\left(p_{1, n+1}\right)^{*} \alpha \cdot\left(p_{2, n+2}\right)^{*} \alpha \cdot \ldots \cdot\left(p_{n, 2 n}\right)^{*} \alpha$, where $p_{i, j}: A^{2 n} \rightarrow A \times A$ are the projections on the $i^{\text {th }}$ and $j^{\text {th }}$ factors for $1 \leq i, j \leq 2 n$. Then, with those conventions, the cycles $p_{S^{n}} \circ \pi_{-}^{\otimes n}$ and $p_{\Lambda^{n}} \circ \pi_{+}^{\otimes n}$ become homologically trivial for $n>>0$. Being symmetrically distinguished, they are also rationally trivial for $n>>0$ by O'Sullivan's Theorem 7.1, thereby establishing Kimura-O'Sullivan's finite-dimensionality for the Chow motive of abelian varieties. 


\section{Multiplicative Chow-Künneth Decompositions}

Let $F$ be a hyperkähler variety. A Fourier transform with kernel a cycle representing the BeauvilleBogomolov class acts as zero on the orthogonal complement of the image of $\operatorname{Sym}^{*} \mathrm{H}^{2}(F, \mathbb{Q})$ inside $\mathrm{H}^{*}(F, \mathbb{Q})$. In general, this orthogonal complement is non-trivial. Thus, such a Fourier transform cannot induce a bigrading on the total Chow ring of $F$ in general. However, it seems reasonable to expect the Chow ring of hyperkähler varieties to actually be endowed with a bigrading that is induced by a ChowKünneth decomposition (as in Conjecture 4). In this section, we introduce the notion of multiplicative Chow-Künneth decomposition ( $c f$. Definition 8.1), discuss its relevance and links with degenerations of modified diagonals ( $c f$. Definition 8.8), and give first examples of varieties that admit a multiplicative Chow-Künneth decomposition. We also provide a proof for Theorem 5 ; see Theorem 8.18. The results of this section will be used in Section 13 to show that the Hilbert scheme of length-2 subschemes on a K3 surface admits a multiplicative Chow-Künneth decomposition, as well as to prove Theorem 6 .

\subsection{Multiplicative Chow-Künneth decompositions : definition and first properties.}

Definition 8.1. Let $X$ be a smooth projective variety of dimension $d$ endowed with a Chow-Künneth decomposition $\left\{\pi^{i}: 0 \leq i \leq 2 d\right\}$ as defined in $\S 3$. The small diagonal $\Delta_{123}$ is the class in $\mathrm{CH}_{d}(X \times$ $X \times X)$ of the image of the diagonal embedding $X \hookrightarrow X \times X \times X, x \mapsto(x, x, x)$. The Chow-Künneth decomposition $\left\{\pi^{i}\right\}$ is said to be

- multiplicative if, for all $k \neq i+j, \pi^{k} \circ \Delta_{123} \circ\left(\pi^{i} \otimes \pi^{j}\right)=0 \in \mathrm{CH}_{d}(X \times X \times X)$ or equivalently if $\Delta_{123}=\sum_{i+j=k} \pi^{k} \circ \Delta_{123} \circ\left(\pi^{i} \otimes \pi^{j}\right) \in \mathrm{CH}_{d}(X \times X \times X)$;

- weakly multiplicative if, for all $k \neq i+j, \pi^{k} \circ \Delta_{123} \circ\left(\pi^{i} \otimes \pi^{j}\right)$ acts as zero on decomposable cycles, that is, if $\left(\pi^{k} \circ \Delta_{123} \circ\left(\pi^{i} \otimes \pi^{j}\right)\right)_{*}(\alpha \times \beta)=\pi_{*}^{k}\left(\pi_{*}^{i} \alpha \cdot \pi_{*}^{j} \beta\right)=0$ for all $\alpha, \beta \in \mathrm{CH}^{*}(X)$ or equivalently if $\left(\sum_{i+j=k} \pi^{k} \circ \Delta_{123} \circ\left(\pi^{i} \otimes \pi^{j}\right)\right)_{*}(\alpha \times \beta)=\alpha \cdot \beta$ for all $\alpha, \beta \in \mathrm{CH}^{*}(X)$.

Here, by definition, we have set $\pi^{i} \otimes \pi^{j}:=p_{1,3}^{*} \pi^{i} \cdot p_{2,4}^{*} \pi^{j}$, where $p_{r, s}: A^{4} \rightarrow A \times A$ are the projections on the $r^{\text {th }}$ and $s^{\text {th }}$ factors for $1 \leq r, s \leq 4$. In both cases, the Chow ring of $X$ inherits a bigrading

$$
\mathrm{CH}_{\mathrm{CK}}^{i}(X)_{s}:=\pi_{*}^{2 i-s} \mathrm{CH}^{i}(X),
$$

which means that $\mathrm{CH}_{\mathrm{CK}}^{i}(X)_{s} \cdot \mathrm{CH}_{\mathrm{CK}}^{j}(X)_{r} \subseteq \mathrm{CH}_{\mathrm{CK}}^{i+j}(X)_{r+s}$.

Remark 8.2. Let $\mathfrak{h}(X)$ be the Chow motive of $X$. The diagonal embedding morphism $\iota_{\Delta}: X \hookrightarrow X \times X$ induces a multiplication map $\mathfrak{h}(X) \otimes \mathfrak{h}(X) \rightarrow \mathfrak{h}(X)$ and a co-multiplication map $\mathfrak{h}(X)(d) \rightarrow \mathfrak{h}(X) \otimes \mathfrak{h}(X)$. Denote $\mathfrak{h}^{i}(X):=\pi^{i} \mathfrak{h}(X)$. Then that the Chow-Künneth decomposition $\left\{\pi^{i}: 0 \leq i \leq 2 d\right\}$ is multiplicative can be restated motivically as requiring that the multiplication map restricted to $\mathfrak{h}^{i}(X) \otimes \mathfrak{h}^{j}(X)$ factors through $\mathfrak{h}^{i+j}(X)$ for all $i$ and $j$, or that the co-multiplication map restricted to $\mathfrak{h}^{k}(X)(d)$ factors through $\bigoplus_{i+j=k} \mathfrak{h}^{i}(X) \otimes \mathfrak{h}^{j}(X)$ for all $k$.

A first class of varieties that admit a multiplicative Chow-Künneth decomposition is given by abelian varieties :

Example 8.3 (Abelian varieties). As in the previous section, let $A$ be an abelian variety of dimension $d, \hat{A}$ its dual, and $L \in \mathrm{CH}^{1}(A \times \hat{A})$ the Poincaré bundle. By Theorem 7.1 and Proposition 7.3, the cycles $\pi^{i}:=\frac{(-1)^{i}}{i !(2 d-i) !} \hat{L}^{i} \circ L^{2 d-i}, i=0, \ldots, 2 d$, are symmetrically distinguished and define a Chow-Künneth decomposition of $A$. This Chow-Künneth decomposition is multiplicative. Indeed, by Theorem 7.1 again, in order to show that $\pi^{k} \circ \Delta_{123} \circ\left(\pi^{i} \otimes \pi^{j}\right)=0$ for all $k \neq i+j$, it is enough to show that $[L]^{2 d-k} \circ\left[\Delta_{123}^{A}\right] \circ\left(p_{13}^{*}[\hat{L}]^{i} \cdot p_{24}^{*}[\hat{L}]^{j}\right)=0$ in cohomology for all $k \neq i+j$. But then, this is (46).

The following proposition gives a useful criterion for a Chow-Künneth decomposition to be multiplicative.

Proposition 8.4. Let $X$ be a smooth projective variety of dimension d endowed with a Chow-Künneth decomposition $\left\{\pi_{X}^{i}: 0 \leq i \leq 2 d_{X}\right\}$ that is self-dual (i.e., such that ${ }^{t} \pi_{X}^{i}=\pi_{X}^{2 d-i}$ for all $i$ ). Endow $X^{3}$ with the product Chow-Künneth decomposition

$$
\pi_{X^{3}}^{k}:=\sum_{i_{1}+i_{2}+i_{3}=k} \pi_{X}^{i_{1}} \otimes \pi_{X}^{i_{2}} \otimes \pi_{X}^{i_{3}}, \quad 0 \leq k \leq 6 d
$$


Then the Chow-Künneth decomposition $\left\{\pi_{X}^{i}\right\}$ is multiplicative if and only if the small diagonal $\Delta_{123}^{X}$ lies in the degree-zero graded part of $\mathrm{CH}_{d}\left(X^{3}\right)$ for the aforementioned product Chow-Künneth decomposition, that is, if and only if $\left(\pi_{X^{3}}^{4 d}\right)_{*} \Delta_{123}^{X}=\Delta_{123}^{X}$.

Proof. Here, the cycle $\pi_{X}^{i_{1}} \otimes \pi_{X}^{i_{2}} \otimes \pi_{X}^{i_{3}}$ is $p_{1,4}^{*} \pi_{X}^{i_{1}} \cdot p_{2,5}^{*} \pi_{X}^{i_{2}} \cdot p_{3,6}^{*} \pi_{X}^{i_{3}}$, where $p_{r, s}: X^{6} \rightarrow X^{2}$ are the projections on the $r^{\text {th }}$ and $s^{\text {th }}$ factors. The proposition follows at once from the formula

$$
\left(\pi_{X}^{i_{1}} \otimes \pi_{X}^{i_{2}} \otimes \pi_{X}^{i_{3}}\right)_{*} \Delta_{123}^{X}=\pi_{X}^{i_{1}} \circ \Delta_{123}^{X} \circ\left({ }^{t} \pi_{X}^{i_{2}} \otimes{ }^{t} \pi_{X}^{i_{3}}\right)=\pi_{X}^{i_{1}} \circ \Delta_{123}^{X} \circ\left(\pi_{X}^{2 d-i_{2}} \otimes \pi_{X}^{2 d-i_{3}}\right),
$$

where the first equality is (40).

Example 8.5 (Abelian varieties, bis). Let $A$ be an abelian variety of dimension $d$. Here is another proof that $A$ admits a multiplicative Chow-Künneth decomposition. By Deninger-Murre [21], $A$ has a ChowKünneth decomposition that induces the same decomposition on $\mathrm{CH}^{*}(A)$ as the Fourier decomposition of Beauville [8]. In order to prove that this Chow-Künneth decomposition is multiplicative, it is enough to check by Proposition 8.4 that $[n]^{*} \Delta_{123}^{A}=n^{4 d} \Delta_{123}^{A}$, where $[n]: A \times A \times A \rightarrow A \times A \times A$ is the multiplication-by- $n$ map. But then, this follows simply from the fact that $[n]$ restricted to the small diagonal is $n^{2 d}$-to- 1 .

The notion of multiplicative Chow-Künneth decomposition is stable under product :

Theorem 8.6. Let $X$ and $Y$ be two smooth projective varieties of respective dimension $d_{X}$ and $d_{Y}$ each endowed with a multiplicative Chow-Künneth decomposition $\left\{\pi_{X}^{i}: 0 \leq i \leq 2 d_{X}\right\}$ and $\left\{\pi_{Y}^{j}: 0 \leq j \leq 2 d_{Y}\right\}$. Then the product Chow-Künneth decomposition on $X \times Y$, which is defined as

$$
\left\{\pi_{X \times Y}^{k}:=\sum_{k=i+j} \pi_{X}^{i} \otimes \pi_{Y}^{j}: 0 \leq k \leq 2\left(d_{X}+d_{Y}\right)\right\}
$$

is multiplicative.

Proof. Let $p_{X}: X \times Y \times X \times Y \times X \times Y \rightarrow X \times X \times X$ be the projection on the first, third and fifth factors, and let $p_{Y}$ denote the projection on the second, fourth and sixth factors. Writing $\Delta_{123}^{X}$ for the small diagonal of $X$ and similarly for $Y$ and $X \times Y$, we have the identity

$$
\Delta_{123}^{X \times Y}=p_{X}^{*} \Delta_{123}^{X} \cdot p_{Y}^{*} \Delta_{123}^{Y}
$$

We immediately deduce that

$$
\left(\pi_{X \times Y}^{a} \otimes \pi_{X \times Y}^{b} \otimes \pi_{X \times Y}^{c}\right)_{*} \Delta_{123}^{X \times Y}=\sum_{\substack{i+i^{\prime}=a \\ j+j^{\prime}=b \\ k+k^{\prime}=c}} p_{X}^{*}\left[\left(\pi_{X}^{i} \otimes \pi_{X}^{j} \otimes \pi_{X}^{k}\right)_{*} \Delta_{123}^{X}\right] \cdot p_{Y}^{*}\left[\left(\pi_{Y}^{i^{\prime}} \otimes \pi_{Y}^{j^{\prime}} \otimes \pi_{Y}^{k^{\prime}}\right)_{*} \Delta_{123}^{Y}\right] .
$$

By Proposition 8.4, the cycles $\left(\pi_{X}^{i} \otimes \pi_{X}^{j} \otimes \pi_{X}^{k}\right)_{*} \Delta_{123}^{X}$ and $\left(\pi_{Y}^{i^{\prime}} \otimes \pi_{Y}^{j^{\prime}} \otimes \pi_{Y}^{k^{\prime}}\right)_{*} \Delta_{123}^{Y}$ are both non-zero only if $i+j+k=4 d_{X}$ and $i^{\prime}+j^{\prime}+k^{\prime}=4 d_{Y}$. Therefore

$$
\left(\pi_{X \times Y}^{a} \otimes \pi_{X \times Y}^{b} \otimes \pi_{X \times Y}^{c}\right)_{*} \Delta_{123}^{X \times Y}=0 \quad \text { if } a+b+c \neq 4\left(d_{X}+d_{Y}\right) .
$$

Hence, by Proposition 8.4 again, the product Chow-Künneth decomposition on $X \times Y$ is multiplicative.

Before moving on to modified diagonals, we state a proposition that will be useful when dealing with multiplicative Chow-Künneth decompositions.

Proposition 8.7. Let $X$ and $Y$ be smooth projective varieties of dimension $d_{X}$ and $d_{Y}$, respectively. Assume that both $X$ and $Y$ have Chow-Künneth decompositions $\left\{\pi_{X}^{i}: 0 \leq i \leq 2 d_{X}\right\}$ and $\left\{\pi_{Y}^{i}: 0 \leq\right.$ $\left.i \leq 2 d_{Y}\right\}$. Endow $X \times Y$ and $X^{n}$ with the product Chow-Künneth decomposition. Then the following statements are true.

(i) If $p_{1}: X \times Y \rightarrow X$ and $p_{2}: X \times Y \rightarrow Y$ are the two projections, then

$$
p_{1}^{*} \alpha \cdot p_{2}^{*} \alpha^{\prime} \in \mathrm{CH}_{\mathrm{CK}}^{p+p^{\prime}}(X \times Y)_{s+s^{\prime}}
$$

for all $\alpha \in \mathrm{CH}_{\mathrm{CK}}^{p}(X)_{s}$ and $\alpha^{\prime} \in \mathrm{CH}_{\mathrm{CK}}^{p^{\prime}}(Y)_{s^{\prime}}$. In particular, we have

$$
p_{1}^{*} \mathrm{CH}_{\mathrm{CK}}^{p}(X)_{s} \subseteq \mathrm{CH}_{\mathrm{CK}}^{p}(X \times Y)_{s} \quad \text { and } \quad p_{2}^{*} \mathrm{CH}_{\mathrm{CK}}^{p^{\prime}}(Y)_{s^{\prime}} \subseteq \mathrm{CH}_{\mathrm{CK}}^{p^{\prime}}(X \times Y)_{s^{\prime}} .
$$


(ii) If $\left\{\pi_{X}^{i}\right\}$ is self-dual, then $\Delta_{X} \in \mathrm{CH}_{\mathrm{CK}}^{d_{X}}(X \times X)_{0}$.

(iii) If $\left\{\pi_{X}^{i}\right\}$ is multiplicative and self-dual, then the small diagonal embedding $\iota_{\Delta, n}: X \rightarrow X^{n}$ is compatible with the decompositions, namely

$$
\left(\iota_{\Delta, n}\right)^{*} \mathrm{CH}_{\mathrm{CK}}^{p}\left(X^{n}\right)_{s} \subseteq \mathrm{CH}_{\mathrm{CK}}^{p}(X)_{s} \quad \text { and } \quad\left(\iota_{\Delta, n}\right)_{*} \mathrm{CH}_{\mathrm{CK}}^{p}(X)_{s} \subseteq \mathrm{CH}_{\mathrm{CK}}^{p+d_{X}(n-1)}\left(X^{n}\right)_{s} .
$$

In particular, the small diagonal $\left(\iota_{\Delta, n}\right)_{*}[X]$ is contained in $\mathrm{CH}_{\mathrm{CK}}^{d_{X}(n-1)}\left(X^{n}\right)_{0}$.

Proof. By definition, $\alpha$ satisfies $\left(\pi_{X}^{i}\right)_{*} \alpha=0$ for all $i \neq 2 p-s$ and $\alpha^{\prime}$ satisfies $\left(\pi_{Y}^{j}\right)_{*} \alpha^{\prime}=0$ for all $j \neq 2 p^{\prime}-s^{\prime}$. Note that

$$
\pi_{X \times Y}^{k}:=\sum_{i+j=k} \pi_{X}^{i} \otimes \pi_{Y}^{j}=\sum_{i+j=k} p_{13}^{*} \pi_{X}^{i} \cdot p_{24}^{*} \pi_{Y}^{j}
$$

We thus have

$$
\begin{aligned}
\left(\pi_{X \times Y}^{k}\right)_{*}\left(p_{1}^{*} \alpha \cdot p_{2}^{*} \alpha^{\prime}\right) & =\sum_{i+j=k}\left(p_{13}^{*} \pi_{X}^{i} \cdot p_{24}^{*} \pi_{Y}^{j}\right)_{*}\left(p_{1}^{*} \alpha \cdot p_{2}^{*} \alpha^{\prime}\right) \\
& =\sum_{i+j=k} p_{1}^{*}\left(\left(\pi_{X}^{i}\right)_{*} \alpha\right) \cdot p_{2}^{*}\left(\left(\pi_{Y}^{j}\right)_{*} \alpha^{\prime}\right) .
\end{aligned}
$$

Therefore, since $\left(\pi_{X}^{i}\right)_{*} \alpha=0$ unless $i=2 p-s$ and $\left(\pi_{Y}^{j}\right)_{*} \alpha^{\prime}=0$ unless $j=2 p^{\prime}-s^{\prime}$, we get that $\left(\pi_{X \times Y}^{k}\right)_{*}\left(p_{1}^{*} \alpha \cdot p_{2}^{*} \alpha^{\prime}\right)=0$ for all $k \neq 2\left(p+p^{\prime}\right)-\left(s+s^{\prime}\right)$. It follows that $p_{1}^{*} \alpha \cdot p_{2}^{*} \alpha^{\prime} \in \mathrm{CH}_{\mathrm{CK}}^{p+p^{\prime}}(X \times Y)_{s+s^{\prime}}$.

Let us now show that the diagonal $\Delta_{X}$ belongs to $\mathrm{CH}_{\mathrm{CK}}^{d_{X}}(X \times X)_{0}$, or equivalently that $\left(\pi_{X \times X}^{k}\right)_{*} \Delta_{X}=0$ for all $k \neq 2 d_{X}$. A direct computation yields

$$
\begin{aligned}
\left(\pi_{X \times X}^{k}\right)_{*} \Delta_{X} & =\sum_{i+j=k}\left(p_{24}\right)_{*}\left(\left(p_{13}^{*} \pi_{X}^{i} \cdot p_{24}^{*} \pi_{X}^{j}\right) \cdot p_{12}^{*} \Delta_{X}\right) \\
& =\sum_{i+j=k} \pi_{X}^{j} \circ{ }^{t} \pi_{X}^{i} \\
& =\sum_{i+j=k} \pi_{X}^{j} \circ \pi_{X}^{2 d_{X}-i} \\
& = \begin{cases}0, & k \neq 2 d_{X} \\
\Delta_{X}, & k=2 d_{X} .\end{cases}
\end{aligned}
$$

We now prove (iii). By Theorem 8.6, the product Chow-Künneth decomposition on $X^{n}$ is multiplicative for all $n \geq 2$. Note that

$$
\left(\iota_{\Delta, n+1}\right)_{*}[X]=\left(p_{1, \ldots, n}\right)^{*}\left(\left(\iota_{\Delta, n}\right)_{*}[X]\right) \cdot p_{n, n+1}^{*} \Delta_{X} \in \mathrm{CH}_{\mathrm{CK}}^{d_{X}(n-1)}\left(X^{n+1}\right)_{0} \cdot \mathrm{CH}_{\mathrm{CK}}^{d_{X}}\left(X^{n+1}\right)_{0} .
$$

It follows by induction that $\left(\iota_{\Delta, n}\right)_{*}[X] \in \mathrm{CH}_{\mathrm{CK}}^{d_{X}(n-1)}\left(X^{n}\right)_{0}$.

Finally, we show that the decompositions are compatible with small diagonal embeddings. On the one hand, if $\alpha \in \mathrm{CH}_{\mathrm{CK}}^{p}(X)_{s}$, then

$$
\left(\iota_{\Delta, n}\right)_{*} \alpha=p_{1}^{*} \alpha \cdot\left(\iota_{\Delta, n}\right)_{*}[X] \in \mathrm{CH}_{\mathrm{CK}}^{p}\left(X^{n}\right)_{s} \cdot \mathrm{CH}_{\mathrm{CK}}^{d_{X}(n-1)}\left(X^{n}\right)_{0} \subseteq \mathrm{CH}_{\mathrm{CK}}^{p+d_{X}(n-1)}\left(X^{n}\right)_{s} .
$$

On the other hand, if $\beta \in \mathrm{CH}_{\mathrm{CK}}^{p}\left(X^{n}\right)_{s}$, then

$$
\left(\iota_{\Delta, n}\right)_{*}\left(\iota_{\Delta, n}\right)^{*} \beta=\beta \cdot\left(\iota_{\Delta, n}\right)_{*}[X] \in \mathrm{CH}_{\mathrm{CK}}^{p}\left(X^{n}\right)_{s} \cdot \mathrm{CH}_{\mathrm{CK}}^{d_{X}(n-1)}\left(X^{n}\right)_{0} \subseteq \mathrm{CH}_{\mathrm{CK}}^{p+d_{X}(n-1)}\left(X^{n}\right)_{s} .
$$

Note that $\left(\iota_{\Delta, n}\right)_{*}$ is injective because $p_{1} \circ \iota_{\Delta, n}=\operatorname{id}_{X}$. Thus we conclude that $\left(\iota_{\Delta, n}\right)^{*} \beta \in \mathrm{CH}_{\mathrm{CK}}^{p}(X)_{s}$.

8.2. Modified diagonals and multiplicative Chow-Künneth decompositions. Another class of varieties admitting a multiplicative Chow-Künneth decomposition that we have in mind is given by K3 surfaces. If $X$ is a K3 surface and $\mathfrak{o}_{X}$ is the class of a point lying on a rational curve, then the ChowKünneth decomposition $\pi^{0}:=\mathfrak{o}_{X} \times X, \pi^{4}:=X \times \mathfrak{o}_{X}$ and $\pi^{2}:=\Delta_{X}-\pi^{0}-\pi^{2}$ is weakly multiplicative. This is due to the fact proved by Beauville-Voisin [11] that the intersection of any two divisors on $X$ is a multiple of $\mathfrak{o}_{X}$ and was explained in the introduction. We would however like to explain why this is in fact a multiplicative Chow-Künneth decomposition. Proposition 8.14 will show that this boils down to 
the main result of [11] on the vanishing of some "modified diagonal". Let us recall the definition of the modified diagonal $\Delta_{\text {tot }}$ as first introduced by Gross and Schoen [27].

Definition 8.8. Recall that the small diagonal is defined as

$$
\Delta_{123}:=\operatorname{Im}\{X \rightarrow X \times X \times X, x \mapsto(x, x, x)\}
$$

viewed as a cycle on $X \times X \times X$. Let $\mathfrak{o}_{X} \in \mathrm{CH}_{0}(X)$ be a zero-cycle of degree 1 . We define for $\{i, j, k\}=\{1,2,3\}$ the following cycles in $\mathrm{CH}_{d}(X \times X \times X)$

$$
\begin{aligned}
\Delta_{i j} & :=p_{i j}^{*} \Delta_{X} \cdot p_{k}^{*} \mathfrak{o}_{X} ; \\
\Delta_{i} & :=p_{i}^{*}[X] \cdot p_{j}^{*} \mathfrak{o}_{X} \cdot p_{k}^{*} \mathfrak{o}_{X},
\end{aligned}
$$

where $p_{i}: X \times X \times X \rightarrow X$ and $p_{i j}: X \times X \times X \rightarrow X \times X$ are the projections. The modified diagonal (attached to the degree-1 zero-cycle $\mathfrak{o}_{X}$ ) is then the cycle

$$
\Delta_{\text {tot }}:=\Delta_{123}-\Delta_{12}-\Delta_{23}-\Delta_{13}+\Delta_{1}+\Delta_{2}+\Delta_{3} \in \mathrm{CH}_{d}(X \times X \times X) .
$$

Here are two important classes of varieties for which $\Delta_{\text {tot }}$ vanishes modulo rational equivalence.

Theorem 8.9 (Gross-Schoen [27]). If $X$ is a hyperelliptic curve and $\mathfrak{o}_{X}$ is the class of a Weierstrass point, then $\Delta_{\text {tot }}=0$ in $\mathrm{CH}_{1}(X \times X \times X)$.

Theorem 8.10 (Beauville-Voisin [11]). If $X$ is a K3 surface and $\mathfrak{o}_{X}$ is the class of any point lying on a rational curve, then $\Delta_{\text {tot }}=0$ in $\mathrm{CH}_{2}(X \times X \times X)$.

Note that the condition that $\Delta_{\text {tot }}$ vanishes is extremely restrictive. Indeed, if $\operatorname{dim} X>1$, a straightforward computation gives

$$
\left(\Delta_{\text {tot }}\right)_{*}(\alpha \times \beta)=\alpha \cup \beta, \quad \text { for all } \alpha \in \mathrm{H}^{p}(X, \mathbb{Q}), \beta \in \mathrm{H}^{q}(X, \mathbb{Q}), 0<p, q<\operatorname{dim} X .
$$

In particular, if $\operatorname{dim} X>2, \Delta_{\text {tot }}$ never vanishes (consider $\alpha=\beta \in \mathrm{H}^{2}(X, \mathbb{Q})$ the class of an ample divisor) and, if $\operatorname{dim} X=2$, then $\Delta_{\text {tot }}=0$ imposes that $\mathrm{H}^{1}(X, \mathbb{Q})=0$. Even in the case of curves, the vanishing of $\Delta_{\text {tot }}$ is very restrictive ; one may consult [27] and [11] for further discussions. More generally, O'Grady [41] considers a higher order modified diagonal, which is defined as follows. Consider a smooth projective variety $X$ of dimension $d$ endowed with a closed point $\mathfrak{o}_{X}$. For $I \subseteq\{1, \ldots, m\}$, we let

$$
\Delta_{I}^{m}\left(X ; \mathfrak{o}_{X}\right):=\left\{\left(x_{1}, \ldots, x_{m}\right) \in X^{m}: x_{i}=x_{j} \text { if } i, j \in I \text { and } x_{i}=\mathfrak{o}_{X} \text { if } i \notin I\right\} .
$$

The $m^{\text {th }}$ modified diagonal cycle associated to $\mathfrak{o}_{X}$ is the $d$-cycle on $X^{m}$ given by

$$
\Delta_{\text {tot }}^{m}\left(X, \mathfrak{o}_{X}\right):=\sum_{\emptyset \neq I \subseteq\{1,2, \ldots, m\}}(-1)^{m-|I|} \Delta_{I}^{m}\left(X ; \mathfrak{o}_{X}\right) .
$$

One may also define the $m^{\text {th }}$ modified diagonal cycle associated to a zero-cycle of degree 1 on $X$ by proceeding as in Definition 8.8. One can then ask if, given a smooth projective variety $X$, there exists a zero-cycle $\mathfrak{o}_{X} \in \mathrm{CH}_{0}(X)$ of degree 1 and an integer $m>0$ such that $\Delta_{\text {tot }}^{m}\left(X ; \mathfrak{o}_{X}\right)=0 \in \mathrm{CH}_{d}\left(X^{m}\right)$. In the case of abelian varieties, the following theorem answers a question raised by O'Grady. This result was obtained independently by Moonen and Yin [37] by using the Fourier transform on abelian varieties $[8,21]$.

Theorem 8.11. Let $A$ be an abelian variety of dimension $d$. Then $\Delta_{\text {tot }}^{2 d+1}(A ; a)=0$ in $\mathrm{CH}_{d}\left(A^{2 d+1}\right)$ for all closed points $a \in A$.

Proof. By translation it is enough to show that $\Delta_{\text {tot }}^{2 d+1}(A, e)=0$ for $e$ the identity element in $A$. The cycle $\Delta_{\text {tot }}^{2 d+1}(A, e)$ is apparently symmetrically distinguished in the sense of O'Sullivan. Therefore, by O'Sullivan's Theorem 7.1, it is rationally trivial if it is homologically trivial. That it is homologically trivial is proved by O'Grady in [41, Proposition 1.3].

An alternate proof of this Theorem will be given after the next proposition in Remark 8.13.

The significance of modified diagonals is made apparent by the following : 
Proposition 8.12. Let $X$ be a smooth projective variety of dimension d over a field $k$. Assume that $X$ admits a multiplicative self-dual Chow-Künneth decomposition $\left\{\pi^{i}: 0 \leq i \leq 2 d\right\}$ with $\pi^{2 d}=X \times \mathfrak{o}_{X}$ for some degree-1 zero-cycle $\mathfrak{o}_{X}$ on $X$. Then $\Delta_{\text {tot }}^{m}\left(X, \mathfrak{o}_{X}\right)=0$ for all $m \geq 2 d+1$.

Moreover, if $\pi^{1}=0$, then $\Delta_{\mathrm{tot}}^{m}\left(X, \mathfrak{o}_{X}\right)=0$ for all $m \geq d+1$.

Proof. For any $I=\left\{i_{1}, i_{2}, \ldots, i_{r}\right\} \subseteq\{1,2, \ldots, m\}$ we define $\Pi_{I}^{m} \in \mathrm{CH}^{m d}\left(X^{m} \times X^{m}\right)$ as the $m$-fold tensor product of $r$ copies of $\Delta_{X}$ and $m-r$ copies of $\pi^{2 d}$ with the diagonals $\Delta_{X}$ being placed in the $\left(i_{1}, i_{2}, \ldots, i_{r}\right)^{\text {th }}$ position. Then one easily checks that

$$
\left(\Pi_{I}^{m}\right)_{*} \Delta^{m}=\Delta_{I}^{m}\left(X ; \mathfrak{o}_{X}\right), \quad \text { where } \Delta^{m}:=\Delta_{\{1, \ldots, m\}}^{m}\left(X, \mathfrak{o}_{X}\right)=\{(x, x, \ldots, x): x \in X\} .
$$

For example, if $I=\{1,2, \ldots, n\}, 1 \leq n \leq m$, then

$$
\left(\Pi_{\{1,2, \ldots, n\}}^{m}\right)_{*} \Delta^{m}=(\underbrace{\Delta_{X} \otimes \cdots \otimes \Delta_{X}}_{n \text { times }} \otimes \underbrace{\pi^{2 d} \otimes \cdots \otimes \pi^{2 d}}_{m-n \text { times }})_{*} \Delta^{m}=\left\{(\underbrace{x, \ldots, x}_{n \text { times }}, \underbrace{\mathfrak{o}_{X}, \ldots, \mathfrak{o}_{X}}_{m-n \text { times }}): x \in X\right\} .
$$

It follows that the $m^{\text {th }}$ modified diagonal satisfies

$$
\Delta_{\text {tot }}^{m}\left(X, \mathfrak{o}_{X}\right)=\left(\Pi^{m}\right)_{*} \Delta^{m}, \quad \text { where } \Pi^{m}:=\sum_{\emptyset \neq I \subseteq\{1, \ldots, m\}}(-1)^{m-|I|} \Pi_{I}^{m} .
$$

Substituting $\Delta_{X}=\pi^{0}+\cdots+\pi^{2 d}$ into the definition of $\Pi_{I}^{m}$, we see that $\Pi_{I}^{m}$ expands into a sum whose terms are of the form $\pi^{j_{1}} \otimes \pi^{j_{2}} \otimes \cdots \otimes \pi^{j_{m}}$ with $j_{k}=2 d$ for all $k \notin I$.

First we claim, without any assumptions on the smooth projective variety $X$, that $\Pi^{m}$ can be expressed as a sum of terms of the form $\pi^{j_{1}} \otimes \pi^{j_{2}} \otimes \cdots \otimes \pi^{j_{m}}$, where none of the indices $j_{k}, 1 \leq k \leq m$, are equal to $2 d$. For that matter, note that the term

$$
(\underbrace{\pi^{2 d} \otimes \cdots \otimes \pi^{2 d}}_{n \text { times }}) \otimes\left(\pi^{l_{1}} \otimes \cdots \otimes \pi^{l_{m-n}}\right), \quad 0 \leq l_{1}, \ldots, l_{m-n}<2 d \text { and } 1 \leq n<m,
$$

appears in $\Pi_{I}^{m}$ (with coefficient 1 ) if and only if $\{n+1, n+2, \ldots, m\} \subseteq I$. There are $\left(\begin{array}{c}n \\ m-r\end{array}\right)$ subsets $\emptyset \neq I \subseteq\{1, \ldots, m\}$ such that $\{n+1, n+2, \ldots, m\} \subset I$ and $|I|=r$. It follows that the term (49) appears in $\Pi^{m}$ with coefficient

$$
\sum_{r=m-n}^{m}(-1)^{m-r}\left(\begin{array}{c}
n \\
m-r
\end{array}\right)=0
$$

By symmetry, any term $\pi^{j_{1}} \otimes \pi^{j_{2}} \otimes \cdots \otimes \pi^{j_{m}}$ with at least one index $j_{k}$ equal to $2 d$ does not appear in $\Pi^{m}$. The claim is thus settled.

Now, assume that the Chow-Künneth decomposition $\left\{\pi^{i}\right\}$ is multiplicative. By Proposition 8.7(iii) the small diagonal $\Delta^{m}$ belongs to $\mathrm{CH}_{\mathrm{CK}}^{d(m-1)}(X)_{0}$ for the product Chow-Künneth decomposition on $X^{m}$ and hence

$$
\left(\pi^{j_{1}} \otimes \pi^{j_{2}} \otimes \cdots \otimes \pi^{j_{m}}\right)_{*} \Delta^{m}=0, \quad \text { for all } j_{1}+j_{2}+\cdots+j_{m} \neq 2 d(m-1) .
$$

When $m \geq 2 d+1$ (or $m \geq d+1$ if $\pi^{1}=0$ ), the condition $j_{1}+j_{2}+\cdots+j_{m}=2 d(m-1)$ forces at least one index $j_{k}$ to be equal to $2 d$ for $\left(\pi^{j_{1}} \otimes \pi^{j_{2}} \otimes \cdots \otimes \pi^{j_{m}}\right)_{*} \Delta^{m}$ to be possibly non-zero. This yields $\Delta_{\text {tot }}^{m}\left(X, \mathfrak{o}_{X}\right)=\left(\Pi^{m}\right)_{*} \Delta^{m}=0$.

Remark 8.13. Note that by combining Example 8.3 or Example 8.5 with Proposition 8.12, we obtain another proof of Theorem 8.11.

In forthcoming work [48], we show that $\Delta_{\text {tot }}^{g+2}(C, c)=0$ in $\mathrm{CH}_{1}\left(C^{g+2}\right)$ for all curves $C$ of genus $g$ and all closed points $c \in C$. Let us also point out that, for a K3 surface $S$, O'Grady [41] shows that $\Delta_{\text {tot }}^{5}\left(S^{[2]}, \mathfrak{o}\right)=0$, where $\mathfrak{o}$ is any point on $S^{[2]}$ corresponding to a subscheme of length 2 supported at a point lying on a rational curve on $S$. This result can also be seen, via Proposition 8.12, as a consequence of our Theorem 13.4 where we establish the existence of a multiplicative Chow-Künneth decomposition for $S^{[2]}$.

After this digression on higher order modified diagonals, we go back to the more down-to-earth $3^{\text {rd }}$ modified diagonal of Definition 8.8. In both cases covered by Theorem 8.9 and Theorem 8.10, the 
vanishing of the $3^{\text {rd }}$ modified diagonal $\Delta_{\text {tot }}$ is directly related to the existence of a multiplicative ChowKünneth decomposition, as shown in the following proposition ; see also [55, §2.3].

Proposition 8.14. Let $X$ be either a curve or a surface with vanishing irregularity and let $\mathfrak{o}_{X} \in \mathrm{CH}_{0}(X)$ be a zero-cycle of degree 1 . Consider the Chow-Künneth decomposition of $X$ consisting of the 3 mutually orthogonal projectors $\pi^{0}:=\mathfrak{o}_{X} \times X, \pi^{2 d}:=X \times \mathfrak{o}_{X}$ and $\pi^{d}:=\Delta_{X}-\pi^{0}-\pi^{2 d}, d=1$ or 2 . Then this Chow-Künneth decomposition is multiplicative if and only if $\Delta_{\text {tot }}=0$.

Proof. The "if" part of the proposition was already covered by Proposition 8.12. Let us however give a direct proof of the proposition. By Definition 8.1, the Chow-Künneth decomposition is multiplicative if and only if

$$
\Delta_{123}=\sum_{i+j=k} \pi^{k} \circ \Delta_{123} \circ\left(\pi^{i} \otimes \pi^{j}\right) .
$$

Substituting $\pi^{d}=\Delta_{X}-\pi^{0}-\pi^{2 d}$ into the above expression and expanding yields

$$
\begin{aligned}
\Delta_{123}= & -\pi^{0} \circ \Delta_{123} \circ\left(\pi^{0} \otimes \pi^{0}\right)-\Delta_{123} \circ\left(\pi^{2 d} \otimes \pi^{0}\right)-\Delta_{123} \circ\left(\pi^{0} \otimes \pi^{2 d}\right) \\
& +\Delta_{123} \circ\left(\pi^{0} \otimes \Delta_{X}\right)+\Delta_{123} \circ\left(\Delta_{X} \otimes \pi^{0}\right)+\pi^{2 d} \circ \Delta_{123} .
\end{aligned}
$$

In order to conclude it is thus enough to check that the first line of the right-hand side of (50) is $-\left(\Delta_{1}+\Delta_{2}+\Delta_{3}\right)$ and that the second line is $\Delta_{12}+\Delta_{23}+\Delta_{13}$. This can be checked term-by-term by using the formula (47).

Remark 8.15. The notion of multiplicative Chow-Künneth decomposition is much stronger than the notion of weakly multiplicative Chow-Künneth decomposition. For instance, it is clear that every curve has a weakly multiplicative Chow-Künneth decomposition but, by Proposition 8.14 and the discussion following Definition 8.8, the class of curves admitting a multiplicative Chow-Künneth decomposition is restricted.

Other classes of varieties admitting a weakly multiplicative Chow-Künneth decomposition but that should not admit a multiplicative Chow-Künneth decomposition in general are given by smooth hypersurfaces in $\mathbb{P}^{4}$. Indeed, let $X$ be a smooth hypersurface in $\mathbb{P}^{4}$ of degree $d$. Let $\mathfrak{o}_{X}$ be the class of $h^{3} / d$, where $h:=c_{1}\left(\mathcal{O}_{X}(1)\right)$. Then the Chow-Künneth decomposition $\pi^{0}:=\mathfrak{o}_{X} \times X, \pi^{2}:=\frac{1}{d} h \times h^{2}, \pi^{4}:=$ $\frac{1}{d} h^{2} \times h, \pi^{6}:=X \times \mathfrak{o}_{X}$ and $\pi^{3}:=\Delta_{X}-\pi^{0}-\pi^{2}-\pi^{4}-\pi^{6}$ is weakly multiplicative. Indeed, we have $\mathrm{CH}_{\mathrm{CK}}^{i}(X)_{0}=\left\langle h^{i}\right\rangle$ for all $i$, and also $\mathrm{CH}^{1}(X)=\mathrm{CH}_{\mathrm{CK}}^{1}(X)_{0}$. We thus only need to check that $h \cdot \alpha=0$ for all $\alpha \in \mathrm{CH}_{\mathrm{CK}}^{2}(X)_{1}$. But then $d h \cdot \alpha=i^{*} i_{*} \alpha$, where $i: X \hookrightarrow \mathbb{P}^{4}$ is the natural embedding. Thus $h \cdot \alpha$ is numerically trivial and proportional to $h^{3}$, and so $h \cdot \alpha=0$. In the case where $X$ is Calabi-Yau, similar arguments as in the proof of Proposition 8.14 show that the Chow-Künneth decomposition above is multiplicative if and only if [55, Conjecture 3.5] holds.

From Proposition 8.14, we deduce the following two examples of varieties having a multiplicative Chow-Künneth decomposition.

Example 8.16 (Hyperelliptic curves). Let $X$ be a hyperelliptic curve and let $\mathfrak{o}_{X}$ be the class of a Weierstrass point. Then the Chow-Künneth decomposition $\pi^{0}:=\mathfrak{o}_{X} \times X, \pi^{2}:=X \times \mathfrak{o}_{X}$ and $\pi^{1}:=$ $\Delta_{X}-\pi^{0}-\pi^{2}$ is multiplicative.

Example 8.17 (K3 surfaces). Let $X$ be a K3 surface and let $\mathfrak{o}_{X}$ be the class of a point lying on a rational curve. Then the Chow-Künneth decomposition $\pi^{0}:=\mathfrak{o}_{X} \times X, \pi^{2}:=X \times \mathfrak{o}_{X}$ and $\pi^{1}:=\Delta_{X}-\pi^{0}-\pi^{2}$ is multiplicative.

8.3. On Theorem 5. Let us finally prove a more precise version of Theorem 5 , which shows that part of Conjecture 4 for hyperkähler varieties of $\mathrm{K}^{[2]}$-type reduces to Conjecture 3 .

Theorem 8.18. Let $F$ be a hyperkähler variety of $\mathrm{K}^{[2]}$-type endowed with a cycle $L \in \mathrm{CH}^{2}(F \times F)$ representing the Beauville-Bogomolov class $\mathfrak{B}$. Assume that $F$ satisfies the following weaker version of Conjecture 3 : the restriction of the cycle class map $\bigoplus_{n} \mathrm{CH}^{*}\left(F^{n}\right) \rightarrow \bigoplus_{n} \mathrm{H}^{*}\left(F^{n}, \mathbb{Q}\right)$ to $V(F ; L)$ is injective. Then $\mathrm{CH}^{*}(F)$ admits a Fourier decomposition as in Theorem 2 which is compatible with its ring structure. Moreover, $F$ admits a multiplicative Chow-Künneth decomposition. 
Proof. By Theorem 2.4, in order to obtain a Fourier decomposition for $\mathrm{CH}^{*}(F)$ as in the statement of Theorem 2, one needs only to check the existence of $L$ satisfying conditions (6), (7), (8) and (9). Note that composition of correspondences only involves intersection products, push-forward and pull-back along projections. Therefore, if as is assumed $V(F ; L)$ injects into cohomology, then one needs only to check that $\mathfrak{B}$ satisfies $(5), \mathfrak{B}_{*} \mathfrak{b}^{2}=0, \mathfrak{B} \circ\left(\mathfrak{b}_{1} \cdot \mathfrak{B}\right)=25 \mathfrak{B}$ and $\left(\mathfrak{B}^{2}\right) \circ\left(\mathfrak{b}_{1} \cdot \mathfrak{B}^{2}\right)=0$. All of these can be read off the results of $\S 1$. As before, note that the intersection product is induced by pulling back along diagonals. By Lemma 6.4, in order to check that the Fourier decomposition is compatible with the intersection product on $\mathrm{CH}^{*}(F)$, one needs only to check a cohomological statement, namely $\mathfrak{B}^{t} \circ\left[\Delta_{123}^{F}\right] \circ\left(p_{13}^{*} \mathfrak{B}^{p} \cdot p_{24}^{*} \mathfrak{B}^{q}\right)=0$ for all $t+p+q \neq 4$. But then, this follows again from the cohomological description of the powers of $\mathfrak{B}$ given in $\S 1$. Finally, under our assumptions, the projectors defined in Proposition 1.7 define ChowKünneth projectors for $F$ and the fact that they define a multiplicative Chow-Künneth decomposition follows from the cohomological equation above : $\mathfrak{B}^{t} \circ\left[\Delta_{123}^{F}\right] \circ\left(p_{13}^{*} \mathfrak{B}^{p} \cdot p_{24}^{*} \mathfrak{B}^{q}\right)=0$ for all $t+p+q \neq 4$.

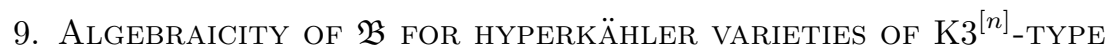

In this section, we use a twisted sheaf constructed by Markman [35] to show that the BeauvilleBogomolov class $\mathfrak{B}$ lifts to a cycle $L \in \mathrm{CH}^{2}(F \times F)$ for all projective hyperkähler manifolds $F$ deformation equivalent to $\mathrm{K} 3^{[n]}, n \geq 2$. The proof goes along these lines: thanks to the work of many authors (cf. Theorem 9.6), one may associate to any K3 surface $S$ endowed with a Mukai vector $v$ a variety $\mathcal{M}$ called the moduli space of stable sheaves with respect to $v$. The variety $\mathcal{M}$ turns out to be a hyperkähler variety of $\mathrm{K} 3^{[n]}$-type. The variety $\mathcal{M} \times S$ comes equipped with a so-called "universal twisted sheaf" $\mathscr{E}$, from which one naturally produces a twisted sheaf $\mathscr{M}$ on $\mathcal{M} \times \mathcal{M}$. The idea behind twisted sheaves is that a sheaf in the usual sense might not deform in a family, while it could deform once it gets suitably "twisted". The main result of Markman [35], which is stated as Theorem 9.12, stipulates that given a hyperkähler variety $F$ of $\mathrm{K} 3^{[n]}$-type, there exists a K3 surface $S$ and a Mukai vector $v$ such that the twisted sheaf $\mathscr{M}$ over the self-product $\mathcal{M} \times \mathcal{M}$ of the associated moduli space $\mathcal{M}$ of stable sheaves deforms to a twisted sheaf on $F \times F$. Furthermore, Markman shows the existence of characteristic classes $(\kappa$-classes) for twisted sheaves. Our sole contribution then consists in computing the cohomology classes of the $\kappa$-classes of the twisted sheaf on $F \times F$; see Proposition 9.11, Lemma 9.14 and Theorem 9.15.

9.1. Twisted sheaves and $\kappa$-classes. Let $Y$ be a compact complex manifold. In [35], Markman defined the classes $\kappa_{i}^{\text {top }}(\mathscr{E}) \in \mathrm{H}^{2 i}(Y, \mathbb{Q})$ for (twisted) sheaves $\mathscr{E}$ on $Y$. We will see that when $Y$ is algebraic the classes $\kappa_{i}(\mathscr{E})$ can be defined at the level of Chow groups, lifting the topological classes.

Assume that $Y$ is projective. Let $K^{0}(Y)$ be the Grothendieck group of locally free coherent sheaves on $Y$, i.e., the free abelian group generated by locally free coherent sheaves on $Y$ modulo the equivalence generated by short exact sequences. Let $y \in K^{0}(Y)$ be an element with nonzero rank $r$, we define

$$
\kappa(y):=\operatorname{ch}(y) \cdot \exp \left(-c_{1}(y) / r\right) \in \mathrm{CH}^{*}(Y) .
$$

We use $\kappa_{i}(y)$ to denote the component of $\kappa(y)$ in $\mathrm{CH}^{i}(Y)$. Note that $\kappa_{1}(y)=0$ for all $y \in K^{0}(Y)$ with nonzero rank. We also easily see that $\kappa$ is multiplicative but not additive. We also have $\kappa(\mathscr{L})=1$ for all invertible sheaves $\mathscr{L}$ on $Y$.

Keep assuming that $Y$ is projective. Let $K_{0}(Y)$ be the Grothendieck group of coherent sheaves on $Y$. Since $Y$ is smooth, every coherent sheaf can be resolved by locally free sheaves and it follows that the natural homomorphism $K^{0}(Y) \rightarrow K_{0}(Y)$ is an isomorphism. This makes it possible to extend the definition of $\kappa$ to (bounded complexes of) coherent sheaves as long as the rank is nonzero. In this case, the multiplicativity of $\kappa$ becomes $\kappa(\mathscr{E} \otimes \mathscr{\mathrm { L }} \mathscr{F})=\kappa(\mathscr{E}) \cdot \kappa(\mathscr{F})$ where $\mathscr{E}$ and $\mathscr{F}$ are (complexes of) coherent sheaves. In particular, we have

$$
\kappa(\mathscr{E} \otimes \mathscr{L})=\kappa(\mathscr{E})
$$

for all invertible sheaves $\mathscr{L}$.

Definition 9.1. Let $Y$ be either a compact complex manifold or a smooth projective variety over an algebraically closed field. Let $\theta \in \mathrm{Br}^{\prime}(X):=\mathrm{H}^{2}\left(Y, \mathcal{O}_{Y}^{*}\right)_{\text {tors }}$, where the topology is either the analytic topology or the étale topology. A $\theta$-twisted sheaf $\mathscr{E}$ consists of the following data. 
(i) An open covering $\mathcal{U}=\left\{U_{\alpha}\right\}_{\alpha \in I}$ of $Y$ in either the analytic or the étale topology ;

(ii) A Cech cocycle $\left\{\theta_{\alpha \beta \gamma}\right\} \in Z^{2}\left(\mathcal{U}, \mathcal{O}_{Y}^{*}\right)$ representing the class $\theta$;

(iii) A sheaf $\mathscr{E}_{\alpha}$ of $\mathcal{O}_{U_{\alpha}}$-modules over $U_{\alpha}$ for each $\alpha \in I$ together with isomorphisms $g_{\alpha \beta}:\left.\mathscr{E}_{\alpha}\right|_{U_{\alpha \beta}} \rightarrow$ $\left.\mathscr{E}_{\beta}\right|_{U_{\alpha \beta}}$ satisfying the conditions : (a) $g_{\alpha \alpha}=\mathrm{id}$, (b) $g_{\alpha \beta}^{-1}=g_{\beta \alpha}$ and (c) $g_{\alpha \beta} g_{\beta \gamma} g_{\gamma \alpha}=\theta_{\alpha \beta \gamma}$ id.

The twisted sheaf $\mathscr{E}$ is locally free (resp. coherent, torsion free or reflexive) if each $\mathscr{E}_{\alpha}$ is. The rank of $\mathscr{E}$ is defined to be the rank of $\mathscr{E}_{\alpha}$. If $\theta=0$, then $\mathscr{E}$ is said to be untwisted.

Remark 9.2. Assume that $Y$ is a smooth projective variety. If $\theta=0$, so that $\mathscr{E}$ is an untwisted sheaf, then $\theta_{\alpha \beta \gamma}$ is a coboundary (after a refinement of the covering if necessary) and hence we can find $h_{\alpha \beta} \in \mathcal{O}_{U_{\alpha \beta}}^{*}$ such that

$$
\theta_{\alpha \beta \gamma}^{-1}=h_{\alpha \beta} h_{\beta \gamma} h_{\gamma \alpha} .
$$

If we modify $g_{\alpha \beta}$ by $h_{\alpha \beta}$ for all $\alpha$ and all $\beta$ and take $\tilde{g}_{\alpha \beta}:=g_{\alpha \beta} h_{\alpha \beta}$, then we may use $\tilde{g}_{\alpha \beta}$ as transition functions and glue the $\mathscr{E}_{\alpha}$ to a global coherent sheaf $\tilde{\mathscr{E}}$. The ambiguity of the choice of $h_{\alpha \beta}$ is a transition function of some line bundle on $Y$. Hence $\tilde{\mathscr{E}}$ is defined up to tensoring with a line bundle on $Y$. Then equation (52) implies that the class $\kappa(\tilde{\mathscr{E}})$ is independent of the choices. We define $\kappa(\mathscr{E}):=\kappa(\tilde{\mathscr{E}})$. By abuse of notation, we will again use $\mathscr{E}$ to denote the sheaf $\tilde{\mathscr{E}}$. Hence when $\mathscr{E}$ is untwisted, we automatically view $\mathscr{E}$ as a usual sheaf of $\mathcal{O}_{Y}$-modules.

Let $\mathscr{E}=\left(U_{\alpha}, \mathscr{E}_{\alpha}, g_{\alpha \beta}\right)$ and $\mathscr{E}^{\prime}=\left(U_{\alpha}, \mathscr{E}_{\alpha}^{\prime}, g_{\alpha \beta}^{\prime}\right)$ be two $\theta$-twisted sheaves. We can define a homomorphism $f: \mathscr{E} \rightarrow \mathscr{E}^{\prime}$ to be a collection of homomorphisms $f_{\alpha}: \mathscr{E}_{\alpha} \rightarrow \mathscr{E}_{\alpha}^{\prime \prime}$ such that $f_{\beta} \circ g_{\alpha \beta}=g_{\alpha \beta}^{\prime} \circ f_{\alpha}$. Then one can naturally define exact sequences of $\theta$-twisted sheaves and the K-group $K_{0}(Y, \theta)$ of $\theta$-twisted sheaves.

With the above remark, it makes sense to ask if $\kappa$ can be defined for twisted sheaves. The following proposition gives a positive answer.

Proposition 9.3 (Markman [35]). Let $Y$ be a smooth projective variety; the function $\kappa$ can be naturally extended to $K_{0}(Y, \theta)$.

Sketch of proof. Assume that $\theta$ has order $r$ and $\mathscr{E}$ is a $\theta$-twisted sheaf. Then, with the correct definition, the $r^{\text {th }}$ tensor product of $\mathscr{E}$ is untwisted and hence $\kappa$ is defined on this tensor product. Then $\kappa(\mathscr{E})$ is defined by taking the $r^{\text {th }}$ root. See $\S 2.2$ of [35] for more details.

Definition 9.4. Let $\mathcal{X} \rightarrow B$ be a flat family of compact complex manifolds (or projective algebraic varieties) and $\theta \in \mathrm{Br}^{\prime}(\mathcal{X})$. A flat family of $\theta$-twisted sheaves is a $\theta$-twisted coherent sheaf $\mathscr{E}$ on $\mathcal{X}$ which is flat over $B$.

Note that for all $t \in B$ the restriction of $\mathscr{E}$ to the fiber $\mathcal{X}_{t}$ gives rise to a $\theta_{t}$-twisted sheaf $\mathscr{E}_{t}$. Usually we do not specify the class $\theta$ and call $\mathscr{E}$ a flat family of twisted sheaves. It is a fact that $\kappa^{\text {top }}\left(\mathscr{E}_{t}\right)$ varies continuously, which we state as the following lemma.

Lemma 9.5. Let $\mathscr{E}$ be a flat family of twisted sheaves on a flat family $\pi: \mathcal{X} \rightarrow B$ of compact complex manifolds, then $\kappa_{i}^{\text {top }}\left(\mathscr{E}_{t}\right) \in \mathrm{H}^{2 i}\left(\mathcal{X}_{t}, \mathbb{Q}\right), t \in B$, are parallel transports of each other in this family.

Proof. We may assume that $B$ is smooth and hence that $\mathcal{X}$ is smooth. By taking some (derived) tensor power of $\mathscr{E}$, we get a complex $\mathscr{E}^{\prime}$ of untwisted locally free sheaves. The $\kappa$-class of $\mathscr{E}$ is a $r^{\text {th }}$-root of the $\kappa$ class of $\mathscr{E}^{\prime}$; see $\S 2.2$ of [35]. By flatness, the above tensor power construction commutes with base change. Hence $\kappa^{\text {top }}\left(\mathscr{E}_{t}\right)$ agrees with the $r^{\text {th }}$-root of $\kappa^{\text {top }}\left(\mathscr{E}_{t}^{\prime}\right)$. As a consequence, we have $\kappa^{\text {top }}\left(\mathscr{E}_{t}\right)=\left.\kappa^{\text {top }}(\mathscr{E})\right|_{\mathcal{X}_{t}}$. This proves the lemma.

9.2. Moduli space of stable sheaves on a K3 surface. The concept of a twisted sheaf naturally appears when one tries to construct universal sheaves over various moduli spaces of stable sheaves. We will be mainly interested in the geometry of moduli spaces of stable sheaves on K3 surfaces ; our main reference is [31].

Let $S$ be an algebraic K3 surface. Let

$$
\tilde{\mathrm{H}}(S, \mathbb{Z})=\mathrm{H}^{0}(S, \mathbb{Z}) \oplus \mathrm{H}^{2}(S, \mathbb{Z}) \oplus \mathrm{H}^{4}(S, \mathbb{Z})
$$


be the Mukai lattice of $S$ endowed with the Mukai pairing

$$
\left(v, v^{\prime}\right):=\int_{S}-v_{0} \cup v_{2}^{\prime}+v_{1} \cup v_{1}^{\prime}-v_{2} \cup v_{0}^{\prime},
$$

for all $v=\left(v_{0}, v_{1}, v_{2}\right)$ and $v^{\prime}=\left(v_{0}^{\prime}, v_{1}^{\prime}, v_{2}^{\prime}\right)$ in $\tilde{\mathrm{H}}(S, \mathbb{Z})$. Given a coherent sheaf $\mathscr{E}$ on $S$, its Mukai vector $v(\mathscr{E})$ is defined to be

$$
v(\mathscr{E}):=\operatorname{ch}^{\text {top }}(\mathscr{E}) \sqrt{\operatorname{td}^{\text {top }}(S)} \in \tilde{\mathrm{H}}(S, \mathbb{Z}) .
$$

For a Mukai vector $v=\left(v_{0}, v_{1}, v_{2}\right)$, we say that $v_{1}$ is primitive if it is not divisible as a cohomology class in $\mathrm{H}^{2}(S, \mathbb{Z})$. Given a Mukai vector $v \in \tilde{\mathrm{H}}(S, \mathbb{Z})$, there is a chamber structure with respect to $v$ on the ample cone of $S$; see [31, Appendix 4.C]. An ample divisor $H$ on $S$ is said to be v-generic if it is contained in an open chamber with respect to $v$. Given $v \in \tilde{\mathrm{H}}(S, \mathbb{Z})$ and an ample divisor $H$ on $S$, we define $\mathcal{M}_{H}(v)$ to be the moduli space of $H$-stable sheaves with Mukai vector $v$. The following result is due to many authors ; see [31, Theorem 6.2.5] and the discussion thereafter.

Theorem 9.6 (Mukai, Huybrechts, Göttsche, O'Grady and Yoshioka). Let $v_{1}$ be primitive and let $H$ be $v$ generic, then $\mathcal{M}_{H}(v)$ is a projective hyperkähler manifold deformation equivalent to $S^{[n]}$ for $n=1+\frac{(v, v)}{2}$.

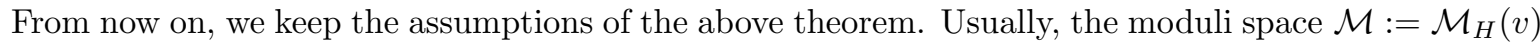
is not fine. Namely there is no universal sheaf on $\mathcal{M} \times S$. However, if we allow twisted sheaves, it turns out that there is always a twisted universal sheaf. We use $\pi_{\mathcal{M}}: \mathcal{M} \times S \rightarrow \mathcal{M}$ and $\pi_{S}: \mathcal{M} \times S \rightarrow S$ to denote the projections.

Proposition 9.7 ([17, Proposition 3.3.2 and 3.3.4]). There exist a unique element $\theta \in \operatorname{Br}^{\prime}(\mathcal{M})$ and a $\pi_{\mathcal{M}}^{*} \theta$-twisted universal sheaf $\mathscr{E}$ on $\mathcal{M} \times S$.

Let $p_{i j}$ be the projections from $\mathcal{M} \times \mathcal{M} \times S$ to the product of the $i^{\text {th }}$ and $j^{\text {th }}$ factors. Then the relative extension sheaf $\mathscr{E} x t_{p_{12}}^{i}\left(p_{13}^{*} \mathscr{E}, p_{23}^{*} \mathscr{E}\right), i \geq 0$, becomes a $\left(p_{1}^{*} \theta^{-1} p_{2}^{*} \theta\right)$-twisted sheaf on $\mathcal{M} \times \mathcal{M}$ where $p_{1}$ and $p_{2}$ are the projections of $\mathcal{M} \times \mathcal{M}$ onto the corresponding factors. For our purpose, the sheaf $\mathscr{E} x t_{p_{12}}^{1}\left(p_{13}^{*} \mathscr{E}, p_{23}^{*} \mathscr{E}\right)$ will be the most important. We will restrict ourselves to the case when $\mathscr{E}$ is untwisted.

Lemma 9.8. Assume that $\mathscr{E}$ is a universal sheaf on $\mathcal{M} \times S$. Then both sheaves $\mathscr{E} x t_{p_{12}}^{0}\left(p_{13}^{*} \mathscr{E}, p_{23}^{*} \mathscr{E}\right)$ and $\mathscr{E} x t_{p_{12}}^{2}\left(p_{13}^{*} \mathscr{E}, p_{23}^{*} \mathscr{E}\right)$ are supported on the diagonal of $\mathcal{M} \times \mathcal{M}$. For any point $t \in \mathcal{M}$, let $\mathscr{E}_{t}$ be the corresponding stable sheaf on $S$. Then both $\mathscr{E} x t_{\pi_{\mathcal{M}}}^{0}\left(\mathscr{E}, \pi_{S}^{*} \mathscr{E}_{t}\right)$ and $\mathscr{E} x t_{\pi_{\mathcal{M}}}^{2}\left(\mathscr{E}, \pi_{S}{ }^{*} \mathscr{E}_{t}\right)$ are supported at the point $t$.

Proof. Let $E_{1}$ and $E_{2}$ be two stable sheaves on $S$, then

$$
\operatorname{Hom}\left(E_{1}, E_{2}\right)= \begin{cases}0, & E_{1} \nsucceq E_{2} ; \\ \mathbb{C}, & E_{1} \cong E_{2} .\end{cases}
$$

This implies that $\mathscr{E} x t_{p_{12}}^{0}\left(p_{13}^{*} \mathscr{E}, p_{23}^{*} \mathscr{E}\right)$ is supported on the diagonal. By Grothendieck-Serre duality, we have $\operatorname{Ext}^{2}\left(E_{1}, E_{2}\right) \cong \operatorname{Hom}\left(E_{2}, E_{1}\right)^{\vee}$. It follows that $\mathscr{E} x t_{p_{12}}^{2}\left(p_{13}^{*} \mathscr{E}, p_{23}^{*} \mathscr{E}\right)$ is also supported on the diagonal. The second half of the lemma is proved similarly.

Here we recall a result of O'Grady [40]. Let $v \in \tilde{\mathrm{H}}(S, \mathbb{Z})$ with $(v, v)>0$. Let

$$
V:=v^{\perp} \subset \tilde{\mathrm{H}}(S, \mathbb{Z})
$$

be the orthogonal complement of $v$ with respect to the Mukai pairing. Note that the restriction of the Mukai pairing to $V$ gives a natural bilinear form on $V$. We define

$$
\mu:=\operatorname{ch}^{\text {top }}(\mathscr{E}) \cup \pi_{S}^{*}(1+[p t]) \in \mathrm{H}^{*}(\mathcal{M} \times S, \mathbb{Q}) .
$$

Let $f: \tilde{\mathrm{H}}(S, \mathbb{Z}) \rightarrow \mathrm{H}^{*}(\mathcal{M}, \mathbb{Q})$ be the homomorphism sending a class $\alpha \in \tilde{\mathrm{H}}(S, \mathbb{Z})$ to $\pi_{\mathcal{M}}\left(\mu^{\vee} \cup \pi_{S}^{*} \alpha\right)$. We recall from $[31, \S 6]$ that if $\mu=\sum \mu_{i} \in \bigoplus \mathrm{H}^{2 i}(\mathcal{M} \times S, \mathbb{Q})$, then $\mu^{\vee}:=\sum(-1)^{i} \mu_{i}$. 
Theorem 9.9 (O'Grady [40]). The restriction of $f$ to $V$ followed by the projection to the $\mathrm{H}^{2}(\mathcal{M}, \mathbb{Z})$ component, usually denoted $\theta_{v}$, gives a canonical isomorphism of integral Hodge structures

$$
\theta_{v}: V \rightarrow \mathrm{H}^{2}(\mathcal{M}, \mathbb{Z})
$$

which respects the bilinear pairing on $V$ and the Beauville-Bogomolov form on the right-hand side.

Furthermore, we have the following

Lemma 9.10. The homomorphism $f$ enjoys the following properties.

(i) The $\mathrm{H}^{2}(\mathcal{M})$-component of $f(v)$ is equal to $\tau_{1}$, where $\tau_{1}=-\operatorname{ch}_{1}^{\text {top }}\left(\mathscr{E} x t_{\pi_{\mathcal{M}}}^{1}\left(\mathscr{E}, \pi_{S}^{*} \mathscr{E} t\right)\right)$ for some closed point $t \in \mathcal{M}$;

(ii) The $\mathrm{H}^{0}(\mathcal{M})$-component of $f(v)$ is equal to $2-2 n$ and $f(w)$ has no $\mathrm{H}^{0}(\mathcal{M})$-component for all $w \in V$.

Proof. The proof of the lemma goes along the same lines as the proof of [31, Theorem 6.1.14] so that we omit the details here.

Proposition 9.11. Assume that there is a universal sheaf $\mathscr{E}$ on $\mathcal{M} \times S$. Let $\mathscr{E}^{i}:=\mathscr{E} x t_{p_{12}}^{i}\left(p_{13}^{*} \mathscr{E}, p_{23}^{*} \mathscr{E}\right)$, $i=0,1,2$. Then the component of $\kappa_{2}^{\text {top }}\left(\mathscr{E}^{1}\right)$ in $\mathrm{H}^{2}(\mathcal{M}, \mathbb{Q}) \otimes \mathrm{H}^{2}(\mathcal{M}, \mathbb{Q})$ is equal to $-\mathfrak{B}$.

Proof. Take a basis $\left\{w_{1}, \ldots, w_{23}\right\}$ of $V$ and set $\hat{w}_{i}=\theta_{v}\left(w_{i}\right)$. By the above result of O'Grady, we know that $\left\{\hat{w}_{1}, \ldots, \hat{w}_{23}\right\}$ forms a basis of $\mathrm{H}^{2}(\mathcal{M}, \mathbb{Z})$. Let $\mu=\sum \mu_{i}^{\prime}$ be the decomposition of $\mu$ such that $\mu_{i}^{\prime} \in \mathrm{H}^{2 i}(\mathcal{M}) \otimes \mathrm{H}^{*}(S)$. Let $A=\left(a_{i j}\right)_{23 \times 23}$ be the intersection matrix of the bilinear form on $V$ with respect to the chosen basis, i.e., $a_{i j}=\left(w_{i}, w_{j}\right)$. Note that $A$ is also the matrix representing the BeauvilleBogomolov form on $\mathrm{H}^{2}(\mathcal{M}, \mathbb{Z})$ with respect to the basis $\hat{w}_{i}$. Let $B=\left(b_{i j}\right)=A^{-1}$. The result of O'Grady together with the first part of Lemma 9.10 implies that

$$
\left(\mu_{1}^{\prime}\right)^{\vee}=-\sum_{1 \leq i, j \leq 23} b_{i j} \hat{w}_{i} \otimes w_{j}^{\vee}-\frac{1}{2 n-2} \tau_{1} \otimes v^{\vee}, \quad \mu_{1}^{\prime}=\sum_{1 \leq i, j \leq 23} b_{i j} \hat{w}_{i} \otimes w_{j}+\frac{1}{2 n-2} \tau_{1} \otimes v .
$$

The second part of Lemma 9.10 implies that $\mu_{0}^{\prime}=[\mathcal{M}] \otimes v$. Then we can compute

$$
\begin{aligned}
\nu & :=\operatorname{ch}^{\text {top }}\left(p_{12 !}\left(p_{13}^{!}[\mathscr{E}]^{\vee} \cdot p_{23}^{!}[\mathscr{E}]\right)\right. \\
& =p_{12 *}\left(p_{13}^{*} \operatorname{ch}^{\text {top }}(\mathscr{E})^{\vee} \cup p_{23}^{*} \operatorname{ch}^{\text {top }}(\mathscr{E}) \cup p_{3}^{*} \operatorname{td}_{S}\right) \\
& =p_{12_{*}}\left(p_{13}^{*} \mu^{\vee} \cup p_{23}^{*} \mu\right),
\end{aligned}
$$

where the second equation uses the Grothendieck-Riemann-Roch theorem. Denoting $[\nu]_{i, j}$ the $\mathrm{H}^{i}(\mathcal{M}) \otimes$ $\mathrm{H}^{j}(\mathcal{M})$-component of $[\nu]$, we readily get

$$
\begin{aligned}
{[\nu]_{2,2} } & =p_{12_{*}}\left(p_{12}^{*}\left(\mu_{1}^{\prime}\right)^{\vee} \cup p_{23}^{*} \mu_{1}^{\prime}\right) \\
& =\sum_{i, j=1}^{23} \sum_{i^{\prime}, j^{\prime}=1}^{23} b_{i j} b_{i^{\prime} j^{\prime}} a_{j j^{\prime}} \hat{w}_{i} \otimes \hat{w}_{i^{\prime}}+\frac{1}{2 n-2} \tau_{1} \otimes \tau_{1} \\
& =\sum_{i, i^{\prime}=1}^{23} b_{i i^{\prime}} \hat{w}_{i} \otimes \hat{w}_{i^{\prime}}+\frac{1}{2 n-2} \tau_{1} \otimes \tau_{1} \\
& =\mathfrak{B}+\frac{1}{2 n-2} \tau_{1} \otimes \tau_{1} .
\end{aligned}
$$

Similarly, we find $[\nu]_{0,2}=-[\mathcal{M}] \otimes \tau_{1}$ and $[\nu]_{2,0}=\tau_{1} \otimes[\mathcal{M}]$ from the second part of Lemma 9.10. Note that

$$
p_{12, !}\left(p_{13}^{!}[\mathscr{E}]^{\vee} \cdot p_{23}^{!}[\mathscr{E}]\right)=\sum(-1)^{i}\left[\mathscr{E}^{i}\right] \in K_{0}(\mathcal{M} \times \mathcal{M})
$$

and recall that by Lemma $9.8, \mathscr{E}^{0}$ and $\mathscr{E}^{2}$ do not contribute to $\mathrm{ch}_{1}$ or $\mathrm{ch}_{2}$. Hence we get

$$
\begin{aligned}
& {\left[\operatorname{ch}_{2}^{\text {top }}\left(\mathscr{E}^{1}\right)\right]_{2,2}=-[\nu]_{2,2}=-\mathfrak{B}-\frac{1}{2 n-2} p_{1}^{*} \tau_{1} \cup p_{2}^{*} \tau_{1},} \\
& \operatorname{ch}_{1}^{\text {top }}\left(\mathscr{E}^{1}\right)=-[\nu]_{0,2}-[\nu]_{2,0}=-p_{1}^{*} \tau_{1}+p_{2}^{*} \tau_{1} .
\end{aligned}
$$


Then the $\kappa$-class of $\mathscr{E}^{1}$ can be computed as follows

$$
\begin{aligned}
\kappa^{\mathrm{top}}\left(\mathscr{E}^{1}\right) & =\operatorname{ch}^{\mathrm{top}}\left(\mathscr{E}^{1}\right) \cdot \exp \left(-\frac{c_{1}^{\mathrm{top}}\left(\mathscr{E}^{1}\right)}{2 n-2}\right) \\
& =\left(2 n-2+\operatorname{ch}_{1}^{\mathrm{top}}\left(\mathscr{E}^{1}\right)+\operatorname{ch}_{2}^{\mathrm{top}}\left(\mathscr{E}^{1}\right)+\cdots\right)\left(1-\frac{\operatorname{ch}_{1}^{\mathrm{top}}\left(\mathscr{E}^{1}\right)}{2 n-2}+\frac{\left(\operatorname{ch}_{1}^{\mathrm{top}}\left(\mathscr{E}^{1}\right)\right)^{2}}{2(2 n-2)^{2}}+\cdots\right) .
\end{aligned}
$$

Therefore

$$
\kappa_{2}^{\text {top }}\left(\mathscr{E}^{1}\right)=\operatorname{ch}_{2}^{\text {top }}\left(\mathscr{E}^{1}\right)-\frac{1}{4 n-4}\left(\operatorname{ch}_{1}^{\text {top }}\left(\mathscr{E}^{1}\right)\right)^{2} .
$$

The Lemma follows by taking the $\mathrm{H}^{2}(\mathcal{M}) \otimes \mathrm{H}^{2}(\mathcal{M})$-component.

9.3. Markman's twisted sheaves. Let $F$ be a hyperkähler manifold of $\mathrm{K} 3^{[n]}$-type, i.e., deformation equivalent to the Hilbert scheme of length- $n$ subschemes of a K3 surface, where $n \geq 2$ is an integer. In [35], Markman constructed a twisted sheaf $\mathscr{M}$ on $F \times F$ which is the deformation of some sheaf that is well understood.

Theorem 9.12 (Markman [35]). Let $F$ be a hyperkähler manifold of $\mathrm{K}^{[n]}$-type. There exists a K3 surface $S$ together with a Mukai vector $v \in \tilde{\mathrm{H}}(S, \mathbb{Z})$ with primitive $v_{1}$ and a v-generic ample divisor $H$ such that there is a proper flat family $\pi: \mathcal{X} \rightarrow C$ of compact hyperkähler manifolds satisfying the following conditions :

(i) The curve $C$, which is connected but possibly reducible, has arithmetic genus 0 .

(ii) There exist $t_{1}, t_{2} \in C$ such that $\mathcal{X}_{t_{1}}=F$ and $\mathcal{X}_{t_{2}}=\mathcal{M}_{H}(v)$.

(iii) A universal sheaf $\mathscr{E}$ exists on $\mathcal{M}_{H}(v) \times S$.

(iv) There is a torsion-free reflexive coherent twisted sheaf $\mathscr{G}$ on $\mathcal{X} \times_{C} \mathcal{X}$, flat over $C$, such that $\mathscr{G}_{t_{2}}:=$ $\left.\mathscr{G}\right|_{\mathcal{X}_{2}}$ is isomorphic to $\mathscr{E} x t_{p_{12}}^{1}\left(p_{13}^{*} \mathscr{E}, p_{23}^{*} \mathscr{E}\right)$.

Definition 9.13. The twisted sheaf $\mathscr{G}_{t_{1}}$ on $F \times F$ obtained in Theorem 9.12 will be denoted $\mathscr{M}$ and, although it might depend on choices, it will be referred to as Markman's sheaf.

However, the low-degree cohomological $\kappa$-classes do not depend on choices :

Lemma 9.14. Let $\kappa_{2}^{\text {top }}(\mathscr{M})=\kappa_{2}^{0,4}+\kappa_{2}^{2,2}+\kappa_{2}^{4,0}$ be the decomposition of $\kappa_{2}^{\text {top }}(\mathscr{M})$ into the Künneth components $\kappa_{2}^{i, j} \in \mathrm{H}^{i}(F) \otimes \mathrm{H}^{j}(F)$. Then we have

(i) $\kappa_{2}^{2,2}=-\mathfrak{B}$ in $\mathrm{H}^{4}(F \times F, \mathbb{Q})$;

(ii) $\kappa_{2}^{4,0}=\frac{1}{2} p_{1}^{*}\left(\mathfrak{b}-c_{2}^{\text {top }}(F)\right)$ and $\kappa_{2}^{0,4}=\frac{1}{2} p_{2}^{*}\left(\mathfrak{b}-c_{2}^{\text {top }}(F)\right)$ in $\mathrm{H}^{4}(F \times F, \mathbb{Q})$;

(iii) $\left(\iota_{\Delta}\right)^{*} \kappa_{2}^{\text {top }}(\mathscr{M})=-c_{2}^{\text {top }}(F)$ in $\mathrm{H}^{4}(F, \mathbb{Q})$, where $\iota_{\Delta}: F \rightarrow F \times F$ is the diagonal embedding.

Proof. Since $\mathscr{M}$ deforms to the sheaf $\mathscr{E}^{1}=\mathscr{E} x t_{p_{12}}^{1}\left(p_{13}^{*} \mathscr{E}, p_{23}^{*} \mathscr{E}\right)$ (by Theorem 9.12), we only need to check (i) for $\mathscr{E}^{1}$ which is Proposition 9.11. Statement (ii) follows from [35, Lemma 1.4], and statement (iii) follows from (i) and (ii).

Theorem 9.15. Let $F$ be a projective hyperkähler manifold of $\mathrm{K} 3^{[n]}-$ type, $n \geq 2$. Then there exists $L \in \mathrm{CH}^{2}(F \times F)$ lifting the Beauville-Bogomolov class $\mathfrak{B} \in \mathrm{H}^{4}(F \times F, \mathbb{Q})$.

Proof. Let $e(F)=\operatorname{deg}\left(c_{2 n}(F)\right)$ be the Euler number of $F$. We first define

$$
l:=\frac{2}{e(F)} p_{2, *}\left(\kappa_{2}(\mathscr{M}) \cdot p_{1}^{*} c_{2 n}(F)\right)+c_{2}(F) \in \mathrm{CH}^{2}(F)
$$

which, by (ii) of Lemma 9.14, lifts the class $\mathfrak{b}$. Then we set

$$
L:=-\kappa_{2}(\mathscr{M})+\frac{1}{2} p_{1}^{*}\left(l-c_{2}(F)\right)+\frac{1}{2} p_{2}^{*}\left(l-c_{2}(F)\right) \in \mathrm{CH}^{2}(F \times F) .
$$

By (i) and (ii) of Lemma 9.14, we see that $L$ lifts $\mathfrak{B}$.

Remark 9.16. With definitions as in the proof of Theorem 9.15, the equality $\iota_{\Delta}^{*} L=l$ holds if and only if item (iii) of Lemma 9.14 holds at the level of Chow groups. 
Remark 9.17. Let $S$ be a K3 surface and let $F=S^{[n]}$ be the Hilbert scheme of length- $n$ subschemes of $S$. Let $Z \subset F \times S$ be the universal family of length- $n$ subschemes of $S$ and let $\mathcal{I}$ be the ideal sheaf defining $Z$ as a subscheme of $F \times S$. In this way, $F$ is realized as the moduli space of stable sheaves with Mukai vector $v=(1,0,1-n)$, and $\mathcal{I}$ is the universal sheaf. In this situation, there is no need to deform and Markman's sheaf is simply given by $\mathscr{M}:=\mathscr{E} x t_{p_{12}}^{1}\left(p_{13}^{*} \mathcal{I}, p_{23}^{*} \mathcal{I}\right)$. We can then define, for $F=S^{[n]}$,

$$
L:=-\kappa_{2}(\mathscr{M})+\frac{1}{2} p_{1}^{*}\left(l-c_{2}(F)\right)+\frac{1}{2} p_{2}^{*}\left(l-c_{2}(F)\right) \in \mathrm{CH}^{2}(F \times F),
$$

where $l:=\frac{2}{e(F)} p_{2, *}\left(\kappa_{2}(\mathscr{M}) \cdot p_{1}^{*} c_{2 n}(F)\right)+c_{2}(F)$. This cycle $L$ represents the Beauville-Bogomolov class $\mathfrak{B}$ by virtue of Theorem 9.15. In the case where $F=S^{[2]}$, this cycle will be shown to coincide with the cycle constructed in (92); see Proposition 16.1. 


\section{Part 2. The Hilbert scheme $S^{[2]}$}

\section{Basics on the Hilbert scheme of Length-2 Subschemes on a variety $X$}

Let $X$ be a smooth projective variety of dimension $d$. Let $F=X^{[2]}$ be the Hilbert scheme of length-2 subschemes on $X$ and let

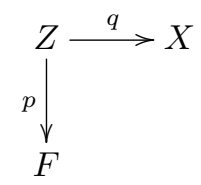

be the universal family seen as a correspondence between $F$ and $X$. For any $w \in F$, we use $Z_{w}:=q\left(p^{-1} w\right)$ to denote the corresponding subscheme of $X$. Note that $Z$ is naturally isomorphic to the blow-up of $X \times X$ along the diagonal. Let $\rho: Z \rightarrow X \times X$ be the blow-up morphism with $E \subset Z$ the exceptional divisor. Then $E$ is naturally identified with the geometric projectivization $\mathbb{P}\left(\mathscr{T}_{X}\right):=\operatorname{Proj}\left(\operatorname{Sym} \Omega_{X}^{1}\right)$ of the tangent bundle $\mathscr{T}_{X}$ of $X$. Let $\pi: E \rightarrow X$ be the natural projection. Then $q$ can be chosen to be $p_{1} \circ \rho$, where $p_{1}: X \times X \rightarrow X$ is the projection onto the first factor. Let $\Delta \subset F$ be the points corresponding to nonreduced subschemes of $X$, or equivalently $\Delta=p(E)$. As varieties, we have $\Delta \cong E$. We will identify $\Delta$ and $E$ with $\mathbb{P}\left(\mathscr{T}_{X}\right)$ and accordingly we have closed immersions $j: \mathbb{P}\left(\mathscr{T}_{X}\right) \hookrightarrow F$ and $j^{\prime}: \mathbb{P}\left(\mathscr{T}_{X}\right) \hookrightarrow Z$. Hence we have the following diagram

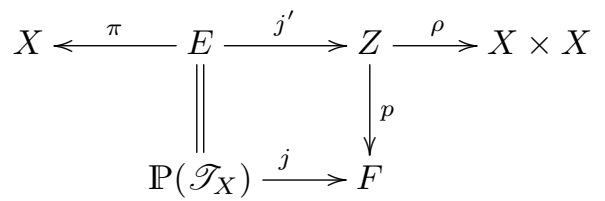

Let $\delta$ be the cycle class of $\frac{1}{2} \Delta$. By abuse of notation, we also use $\delta$ to denote its cohomological class in $\mathrm{H}^{2}(F, \mathbb{Z})$. If we further assume that $\mathrm{H}^{1}(X, \mathbb{Z})=0$, then we see that

$$
\mathrm{H}^{2}(F, \mathbb{Z})=p_{*} q^{*} \mathrm{H}^{2}(X, \mathbb{Z}) \oplus \mathbb{Z} \delta .
$$

We also note that the double cover $Z \rightarrow F$ naturally induces an involution $\tau: Z \rightarrow Z$.

Remark 10.1. If $X=S$ is a $\mathrm{K} 3$ surface, then $F$ is a hyperkähler manifold ; see [6]. The homomorphism $[Z]^{*}: \mathrm{H}^{2}(S, \mathbb{Z}) \rightarrow \mathrm{H}^{2}(F, \mathbb{Z})$ induces the following orthogonal direct sum decomposition with respect to the Beauville-Bogomolov form $q_{F}$ :

$$
\mathrm{H}^{2}(F, \mathbb{Z})=\mathrm{H}^{2}(S, \mathbb{Z}) \hat{\oplus} \mathbb{Z} \delta
$$

Furthermore, $q_{F}(\delta, \delta)=-2$ and $q_{F}$ restricted to $\mathrm{H}^{2}(S, \mathbb{Z})$ is the intersection form on $S$.

For any given point $x \in X$, we can define a smooth variety $X_{x}=p\left(q^{-1} x\right) \subset F$. The variety $X_{x}$ is isomorphic to the blow-up of $X$ at the point $x$ and it represents the cycle $Z^{*} x$. For two distinct points $x, y \in X$, we use $[x, y] \in F$ to denote the point of $F$ that corresponds to the subscheme $\{x, y\} \subset X$. When $x=y$, we use $[x, x]$ to denote the element in $\mathrm{CH}_{0}(F)$ represented by any point corresponding to a nonreduced subscheme of length 2 of $X$ supported at $x$. As cycles, we have

$$
X_{x} \cdot X_{y}=[x, y] .
$$

Intersecting with $\delta$ is unveiled in the following lemma.

Lemma 10.2. Let $\pi: \mathbb{P}\left(\mathscr{T}_{X}\right) \rightarrow X$ be the natural morphism and $\xi \in \mathrm{CH}^{1}\left(\mathbb{P}\left(\mathscr{T}_{X}\right)\right)$ be the first Chern class of the relative $\mathcal{O}(1)$ bundle. Then we have the following identities in $\mathrm{CH}^{*}(F)$,

$$
\begin{aligned}
& \delta^{k}=\frac{(-1)^{k-1}}{2} j_{*} \xi^{k-1}, \quad k=1,2, \ldots, 2 d ; \\
& \delta^{k} \cdot X_{x}=(-1)^{k-1}\left(j_{x}\right)_{*}\left(\xi_{x}^{k-1}\right), \quad k=1,2, \ldots, d,
\end{aligned}
$$


where $j_{x}: \pi^{-1} x=\mathbb{P}\left(\mathscr{T}_{X, x}\right) \rightarrow F$ is the natural closed immersion and $\xi_{x}$ is the class of a hyperplane on $\mathbb{P}\left(\mathscr{T}_{X, x}\right)$. In particular, we have

$$
\delta^{d} \cdot X_{x}=(-1)^{d-1}[x, x] \quad \text { in } \mathrm{CH}_{0}(F) .
$$

Proof. First recall that $p: Z \rightarrow F$ has degree 2 and that $\delta=\frac{1}{2} p_{*} E$. By the projection formula, we get

$$
p_{*}\left(E^{k}\right)=p_{*} p^{*}\left(\delta^{k}\right)=\delta^{k} \cdot p_{*}(Z)=2 \delta^{k} .
$$

Note that $E^{k}=j_{*}^{\prime}(-\xi)^{k-1}=(-1)^{k-1} j_{*}^{\prime} \xi^{k-1}$. It follows that $\delta^{k}=\frac{(-1)^{k-1}}{2} j_{*} \xi^{k-1}, k=1, \ldots, 2 d-1$. Now,

$$
\delta^{k} \cdot X_{x}=\frac{1}{2} p_{*} E^{k} \cdot X_{x}=\frac{1}{2} p_{*}\left(E^{k} \cdot p^{*} X_{x}\right)=\frac{1}{2} p_{*}\left(E^{k} \cdot\left(\tilde{X}_{x}+\tau^{*} \tilde{X}_{x}\right)\right)=p_{*}\left(E^{k} \cdot \widetilde{X}_{x}\right),
$$

where $\widetilde{X}_{x}=q^{-1}(x)$. Note that $E \cdot \widetilde{X}_{x}=\left(j_{x}^{\prime}\right)_{*} \mathbb{P}\left(\mathscr{T}_{X, x}\right)$, where $j_{x}^{\prime}=\left.j^{\prime}\right|_{\pi^{-1} x}$ and that $\left(j_{x}^{\prime}\right)^{*} E=-\xi_{x}$. We get

$$
E^{k} \cdot \widetilde{X}_{x}=E^{k-1} \cdot\left(j_{x}^{\prime}\right)_{*} \mathbb{P}\left(\mathscr{T}_{X, x}\right)=\left(j_{x}^{\prime}\right)_{*}\left(j_{x}^{\prime}\right)^{*}\left(E^{k-1}\right)=(-1)^{k-1}\left(j_{x}^{\prime}\right)_{*}\left(\xi_{x}^{k-1}\right) .
$$

Apply $p_{*}$ to the above equation and the second equality of the lemma follows since $j_{x}=p \circ j_{x}^{\prime}$.

\section{The InCIDENCE CORRESPONDENCE $I$}

Let $X$ be a smooth projective variety of dimension $d$ and let $F:=X^{[2]}$ be the Hilbert scheme of length-2 subschemes on $X$. We introduce the incidence correspondence $I \in \mathrm{CH}^{d}(F \times F)$ and establish a quadratic equation satisfied by $I$; see Proposition 11.4. We also compute, in Lemma 11.7, the pull-back of $I$ along the diagonal embedding $\iota_{\Delta}: F \hookrightarrow F \times F$. The first result will already make it possible to obtain in that generality a splitting of the Chow group of zero-cycles on $X^{[2]}$; see Section 12 . When $X$ is a K3 surface, the incidence cycle $I$ will be closely related to the crucial lifting of the Beauville-Bogomolov class $\mathfrak{B}$, and Proposition 11.4 and Proposition 11.7 will constitute the first step towards establishing the quadratic equation (6) of Conjecture 1 ; see $\$ 14.2$.

The notations are those of the previous section. Consider the diagram

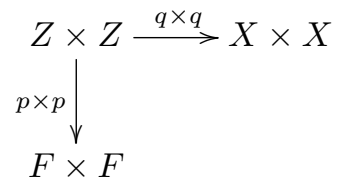

Definition 11.1. The incidence subscheme $I \subset F \times F$ is defined by

$$
I:=\left\{\left(w_{1}, w_{2}\right) \in F \times F \text { : there exists a closed point } x \in X \text { such that } x \in Z_{w_{i}}, i=1,2\right\},
$$

where the right hand side is equipped with the reduced closed subscheme structure. The incidence correspondence is the cycle class of $I$ in $\mathrm{CH}^{2}(F \times F)$ and, by abuse of notations, is also denoted $I$.

The following lemma justifies this abuse of notations.

Lemma 11.2. The incidence subscheme I enjoys the following properties.

(i) The sub-variety I is irreducible and its cycle class is given by

$$
I=(p \times p)_{*}(q \times q)^{*} \Delta_{X} \quad \text { in } \mathrm{CH}^{d}(F \times F) .
$$

Equivalently, if one sees the universal family $Z$ of length-2 subschemes on the variety $X$ as a correspondence between $F=X^{[2]}$ and $X$, then

$$
I={ }^{t} Z \circ Z, \quad \text { in } \mathrm{CH}^{d}(F \times F) .
$$

(ii) The diagonal $\Delta_{F}$ is contained in $I$ and $I \backslash \Delta_{F}$ is smooth.

(iii) The action of $I$ on points is given by

$$
I_{*}[x, y]=X_{x}+X_{y} .
$$


Proof. Recall that $Z$ is the blow-up of $X \times X$ along the diagonal and that $q: Z \rightarrow X$ is the composition of the blow-up morphism $Z \rightarrow X \times X$ with the first projection $p_{1}: X \times X \rightarrow X$. As a consequence, $q$ is a smooth morphism and $q^{-1} x \cong \mathrm{Bl}_{x}(X)$ is the blow-up of $X$ at the closed point $x \in X$. It follows that $q \times q: Z \times Z \rightarrow X \times X$ is smooth. Hence $Z_{\Delta}:=(q \times q)^{-1} \Delta_{X} \subset Z \times Z$ is a smooth sub-variety whose cycle class is $(q \times q)^{*} \Delta_{X}$. Note that the image of $p \times p$ restricted to $Z_{\Delta}$ is $I$. In particular, $I$ is irreducible. To prove (i), it suffices to show that the morphism $\left.(p \times p)\right|_{Z_{\Delta}}$ is generically one-to-one. A general point of $I$ has the form $([x, y],[y, z])$, where $x, y, z \in X$ are distinct points and the inverse image of $([x, y],[y, z])$ in $Z \times Z$ is

$$
\left\{\left(\rho^{-1}(x, y), \rho^{-1}(y, z)\right),\left(\rho^{-1}(y, x), \rho^{-1}(y, z)\right),\left(\rho^{-1}(x, y), \rho^{-1}(z, y)\right),\left(\rho^{-1}(y, x), \rho^{-1}(z, y)\right)\right\} .
$$

Among these four points, the only point contained in $Z_{\Delta}$ is $\left(\rho^{-1}(y, x), \rho^{-1}(y, z)\right)$. Since the degree of $p \times p$ is 4 , this proves that $\left.(p \times p)\right|_{z_{\Delta}}$ has degree 1 and hence statement (i) follows.

To prove (ii), we note that the fiber of the natural morphism $I \rightarrow F$ (projection to the first factor) over a point $[x, y] \in F$ is $[x, y] \times\left(X_{x} \cup X_{y}\right)$, which is smooth away from the point $([x, y],[x, y])$ if $x \neq y$. Hence the singular locus of $I$ is contained in the union of $\Delta_{F}$ and $E \times_{X} E$. Note that a length-2 subscheme supported at a point $x$ is given by a 1-dimensional sub-space $\mathbb{C} v \subset \mathscr{T}_{X, x}$; we use $(x, \mathbb{C} v)$ to denote the corresponding point of $F$. Let $u=\left(\left(x, \mathbb{C} v_{1}\right),\left(x, \mathbb{C} v_{2}\right)\right) \in I$ be a point supported on $E \times_{X} E$. Let $\tilde{u} \in Z \times Z$ be the unique lift of $u$, and let

$$
t \mapsto \varphi_{i}(t) \in X, \quad t \in \mathbb{C},|t|<\epsilon,
$$

be a germ of a smooth analytic curve with $\varphi_{i}^{\prime}(0) \in \mathbb{C} v_{i}, i=1,2$. We define

$$
\tilde{\varphi}_{i}:\{t \in \mathbb{C}:|t|<\epsilon\} \longrightarrow Z
$$

to be the strict transform of the curve

$$
t \mapsto\left(\varphi_{i}(t), \varphi_{i}(-t)\right) \in X \times X .
$$

Let $S \subset Z \times Z$ be the surface parameterized by

$$
\left(t_{1}, t_{2}\right) \mapsto\left(\tilde{\varphi}_{1}\left(t_{1}\right), \tilde{\varphi}_{2}\left(t_{2}\right)\right) \in Z \times Z .
$$

Then $\mathscr{T}_{S, \tilde{u}} \subset \mathscr{T}_{Z \times Z, \tilde{u}}$ is the kernel of $d(p \times p): \mathscr{T}_{Z \times Z, \tilde{u}} \rightarrow \mathscr{T}_{F \times F, u}$. The scheme-theoretic intersection of $Z_{\Delta}$ and $S$ is given by the equation $\varphi_{1}\left(t_{1}\right)=\varphi_{2}\left(t_{2}\right)$. It follows that $S$ intersects $I$ at the point $\tilde{u}$ with multiplicity 1 if $\mathbb{C} v_{1} \neq \mathbb{C} v_{2}$. This implies that the map $\mathscr{T}_{Z_{\Delta}, \tilde{u}} \rightarrow \mathscr{T}_{I, u}$ is an isomorphism. In particular, $I$ is smooth at $u$ if $\mathbb{C} v_{1} \neq \mathbb{C} v_{2}$ or, equivalently, if $u \notin \Delta_{F}$. Hence we conclude that $I \backslash \Delta_{F}$ is smooth. Statement (iii) follows directly from the definition of $I$.

Definition 11.3. Let $\sigma \in \mathrm{CH}^{r}(X)$; we define an element $\Gamma_{\sigma} \in \mathrm{CH}^{d+r}(F \times F)$ as follows.

$$
\Gamma_{\sigma}:=(p \times p)_{*}(q \times q)^{*}\left(\iota_{\Delta_{X}}\right)_{*} \sigma,
$$

where $\iota_{\Delta_{X}}: X \rightarrow X \times X$ is the diagonal embedding.

Note that, if $\sigma$ is represented by an irreducible closed sub-variety $Y \subset X$, then $\Gamma_{\sigma}$ is the cycle class of

$$
\Gamma_{Y}:=\left\{\left(w_{1}, w_{2}\right) \in F \times F: \exists y \in Y, y \in Z_{w_{1}} \text { and } y \in Z_{w_{2}}\right\}
$$

In particular, if we take $Y=X$, then we have $\Gamma_{X}=I$.

The following proposition is the analogue of an identity of Voisin, stated in (105), which was originally proved in the case of varieties of lines on cubic fourfolds.

Proposition 11.4. Let $\delta_{i}, i=1,2$, be the pull-back of $\delta$ via the projection of $F \times F$ to the $i^{\text {th }}$ factor. We write

$$
y_{k}:=(-1)^{k-1}\left(\delta_{1}^{k}-\sum_{i=1}^{k-1} \delta_{1}^{i} \delta_{2}^{k-i}+\delta_{2}^{k}\right), \quad k=1,2, \ldots, 4 d, d=\operatorname{dim} X,
$$

and $y_{0}:=[F \times F]$. Then the cycle I satisfies the following equation in $\mathrm{CH}^{2 d}(F \times F)$,

$$
I^{2}=2 \Delta_{F}+y_{d} \cdot I+\sum_{k=0}^{d-1} y_{k} \cdot \Gamma_{c_{d-k}(X)} .
$$


Proof. To prove this result, we first give a different description of the cycle $I$. Consider the following diagram

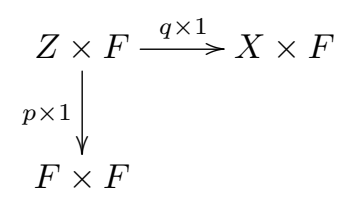

The variety $Z$ is naturally a sub-variety of $X \times F$. Then $I=(p \times 1)_{*}(q \times 1)^{*} Z$. Let $\tilde{I}=(q \times 1)^{-1} Z \subset Z \times F$. Note that $\tilde{I}$ is smooth since $q \times 1$ is a smooth morphism. Then we see that $\tilde{I}$ is a local complete intersection and

$$
\mathscr{N}_{\tilde{I} / Z \times F} \cong\left(q \times\left. 1\right|_{\tilde{I}}\right)^{*} \mathscr{N}_{Z / X \times F}
$$

The morphism $p \times 1$ induces a homomorphism

$$
\vartheta=d(p \times 1): \mathscr{N}_{\tilde{I} / Z \times F} \longrightarrow\left(p \times\left. 1\right|_{\tilde{I}}\right)^{*} \mathscr{N}_{I / F \times F} .
$$

The homomorphism $\vartheta$ fits into the following commutative diagram with short exact rows and columns

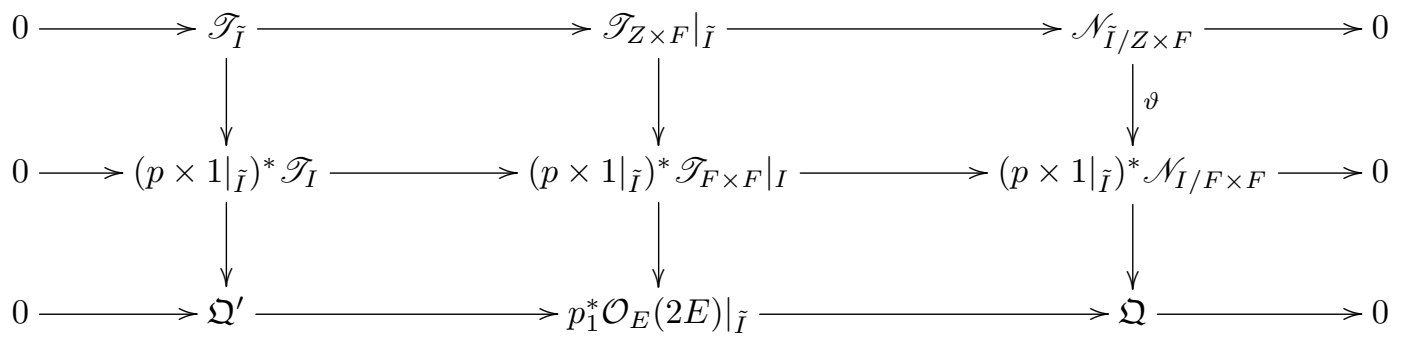

where $p_{i}, i=1,2$, are the projections of $Z \times F$ onto the two factors and all the sheaves are viewed as their restrictions to $\tilde{I} \backslash(p \times 1)^{-1} \Delta_{F}$. In the above diagram, the middle column follows from the following fundamental short exact sequence

$$
0 \longrightarrow \mathscr{T}_{Z} \longrightarrow p^{*} \mathscr{T}_{F} \longrightarrow \mathcal{O}_{E}(2 E) \longrightarrow 0 .
$$

Note that the morphism $p \times\left. 1\right|_{\tilde{I}}: \tilde{I} \rightarrow I$ is an isomorphism away from $\Delta_{F} \subset I ;$ it follows that $\mathfrak{Q}^{\prime}=0$ away from $(p \times 1)^{-1} \Delta_{F}$. Hence a Chern class computation on $\tilde{I} \backslash(p \times 1)^{-1} \Delta_{F}$ gives

$$
\begin{aligned}
c\left(\left(p \times\left. 1\right|_{\tilde{I}}\right)^{*} \mathscr{N}_{I / F \times F}\right) & =\left.c\left(p_{1}^{*} \mathcal{O}_{E}(2 E)\right)\right|_{\tilde{I}} \cdot c\left(\mathscr{N}_{\tilde{I} / Z \times F}\right) \\
& =\left.c\left(p_{1}^{*} \mathcal{O}_{E}(2 E)\right)\right|_{\tilde{I}} \cdot c\left(\left(q \times\left. 1\right|_{\tilde{I}}\right)^{*} \mathscr{N}_{Z / S \times F}\right) .
\end{aligned}
$$

We have the following short exact sequence

$$
0 \longrightarrow \mathcal{O}_{Z}(E) \longrightarrow \mathcal{O}_{Z}(2 E) \longrightarrow \mathcal{O}_{E}(2 E) \longrightarrow \text {. }
$$

From this exact sequence we get

$$
c\left(\mathcal{O}_{E}(2 E)\right)=\frac{1+2 E}{1+E}=1+E-E^{2}+E^{3}-E^{4}+\cdots-E^{2 d}, \quad \text { in } \mathrm{CH}^{*}(Z), d=\operatorname{dim} X .
$$

In order to compute the Chern classes of $\mathscr{N}_{Z / S \times F}$, we consider the following diagram

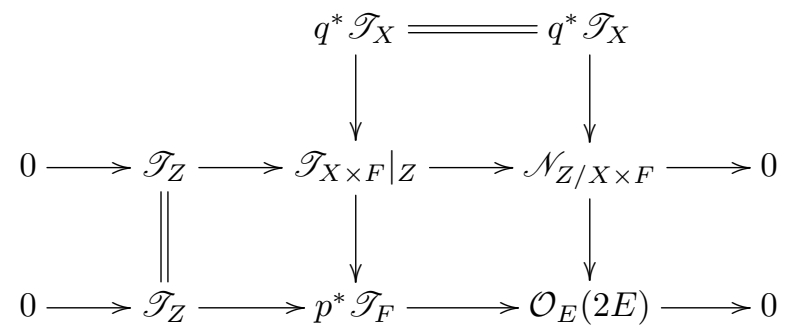

where all the rows and columns are short exact. As a result we have

$$
c\left(\mathscr{N}_{Z / X \times F}\right)=c\left(q^{*} \mathscr{T}_{X}\right) \cdot c\left(\mathcal{O}_{E}(2 E)\right)=q^{*} c(X) \cdot\left(1+E-E^{2}+\cdots-E^{2 d}\right),
$$


where $c(X)=1+c_{1}(X)+\cdots+c_{d}(X)$. Substituting (62) and (63) into (64) yields

$$
\begin{aligned}
c\left(\left(p \times\left. 1\right|_{\tilde{I}}\right)^{*} \mathscr{N}_{I / F \times F}\right)= & \left.p_{1}^{*}\left(1+E-E^{2}+E^{3}-E^{4}+\cdots-E^{2 d}\right)\right|_{\tilde{I}} \cdot\left(q \times\left. 1\right|_{\tilde{I}}\right)^{*} q^{*} c(X) \\
& \cdot\left(q \times\left. 1\right|_{\tilde{I}}\right)^{*}\left(1+E-E^{2}+E^{3}-E^{4}+\cdots-E^{2 d}\right) \\
= & \left.\left.p_{1}^{*} p^{*}\left(1+\delta-\delta^{2}+\delta^{3}-\delta^{4}+\cdots-\delta^{2 d}\right)\right|_{\tilde{I}} \cdot p_{1}^{*} q^{*} c(X)\right|_{\tilde{I}} \\
& \cdot\left(q \times\left. 1\right|_{\tilde{I}}\right)^{*} p^{*}\left(1+\delta-\delta^{2}+\delta^{3}-\delta^{4}+\cdots-\delta^{2 d}\right) \\
= & \left.\left.p_{1}^{*} p^{*}\left(1+\delta-\delta^{2}+\delta^{3}-\delta^{4}+\cdots-\delta^{2 d}\right)\right|_{\tilde{I}} \cdot p_{1}^{*} q^{*} c(X)\right|_{\tilde{I}} \\
& \left.\cdot p_{2}^{*}\left(1+\delta-\delta^{2}+\delta^{3}-\delta^{4}+\cdots-\delta^{2 d}\right)\right|_{\tilde{I}} \\
= & (p \times 1)^{*}\left\{\left(1+\delta_{1}-\delta_{1}^{2}+\delta_{1}^{3}-\delta_{1}^{4}+\cdots-\delta^{2 d}\right)\right. \\
& \left.\cdot\left(1+\delta_{2}-\delta_{2}^{2}+\delta_{2}^{3}-\delta_{2}^{4}+\cdots-\delta^{2 d}\right)\right\}\left.\left.\right|_{\tilde{I}} \cdot p_{1}^{*} q^{*} c(X)\right|_{\tilde{I}},
\end{aligned}
$$

when restricted to $\tilde{I} \backslash(p \times 1)^{-1} \Delta_{F}$. The second equation follows from the fact that $p^{*} \delta=E$ and that the composition $\left.q \circ(q \times 1)\right|_{\tilde{I}}$ is equal to $\left.\left(q \circ p_{1}\right)\right|_{\tilde{I}}$. The third equation holds since the composition of $q \times\left. 1\right|_{\tilde{I}}: \tilde{I} \rightarrow Z$ and $p: Z \rightarrow F$ is simply the second projection $p_{2}: Z \times F \rightarrow F$ restricted to $\tilde{I}$. Now we need the following lemma.

Lemma 11.5. Let $\sigma \in \mathrm{CH}^{r}(X)$ be a cycle. Then

$$
(p \times 1)_{*}\left(p_{1}^{*} q^{*} \sigma \cdot \tilde{I}\right)=\Gamma_{\sigma},
$$

where $p_{1}: Z \times F \rightarrow Z$ is the projection to the first factor and $p \times 1: Z \times F \rightarrow F \times F$ is the natural morphism.

Proof. We may assume that $\sigma$ is represented by an irreducible closed sub-variety $Y \subset X$. Then by definition, the class $p_{1}^{*} q^{*} \sigma \cdot \tilde{I}$ is represented by the cycle

$$
\left\{(z, w) \in Z \times F: q(z) \in Y \text { and } q(z) \in Z_{w}\right\},
$$

whose push-forward to $F \times F$ is easily seen to be $\Gamma_{Y}$; see equation (60).

Then the above equation (65) for $c\left(\left(p \times\left. 1\right|_{\tilde{I}}\right)^{*} \mathscr{N}_{I / F \times F}\right)$ implies

$$
c_{d}\left(\left(p \times\left. 1\right|_{\tilde{I}}\right)^{*} \mathscr{N}_{I / F \times F}\right)=\left.p_{1}^{*} q^{*} c_{n}(X)\right|_{\tilde{I}}+\left.\sum_{k=1}^{d}(p \times 1)^{*} y_{k} \cdot p_{1}^{*} q^{*} c_{d-k}(X)\right|_{\tilde{I}} .
$$

We apply $\left(p \times\left. 1\right|_{\tilde{I}}\right)_{*}$ to the above identity followed by $\left(i_{0}\right)_{*}$, where $i_{0}: I_{0}=I \backslash \Delta_{F} \rightarrow F \times F \backslash \Delta_{F}$ is the natural inclusion. Then we get

$$
\left(i_{0}\right)_{*} c_{d}\left(\mathscr{N}_{I / F \times F}\right)=\sum_{k=0}^{d} y_{k} \cdot(p \times 1)_{*}\left(p_{1}^{*} q^{*} c_{d-k}(X) \cdot \tilde{I}\right), \quad \text { when restricted to } F \times F \backslash \Delta_{F} .
$$

Recall that the self-intersection of $I$ outside of the diagonal is given by the top Chern class of its normal bundle. We then apply the localization sequence for Chow groups and Lemma 11.5 to obtain

$$
I^{2}=\alpha \Delta_{F}+y_{d} \cdot I+\sum_{k=0}^{d-1} y_{k} \cdot \Gamma_{c_{d-k}(X)}
$$

for some $\alpha \in \mathbb{Z}$. In order to determine the integer $\alpha$, we will compute the degree of $\left(I^{2}\right)_{*}[x, y]$ for a general point $[x, y] \in F$. First we have the following lemma.

Lemma 11.6. Let $\tau \in \mathrm{CH}_{0}(F)$, then $\Gamma_{*} \tau=0$ for all $\Gamma=y_{k} \cdot \Gamma_{c_{d-k}(X)}, k=0,1, \ldots, d-1$.

Proof. Since $y_{k}$ is a linear combination of $\delta_{1}^{i} \delta_{2}^{k-i}, i=0,1, \ldots, k$, we only need to show the following

$$
\left(\delta_{1}^{i} \delta_{2}^{k-i} \cdot \Gamma_{c_{d-k}(X)}\right)_{*} \tau=\delta^{k-i} \cdot\left(\Gamma_{c_{d-k}(X)}\right)_{*}\left(\delta^{i} \cdot \tau\right)=0 .
$$

Note that $\delta^{i} \cdot \tau=0$ unless $i=0$. It reduces to showing that $\left(\Gamma_{\sigma}\right)_{*} \tau=0$ for all cycles $\sigma$ on $X$ of dimension less than $d$. Using the definition of $\Gamma_{\sigma}$, we get

$$
p_{1}^{*} \tau \cdot \Gamma_{\sigma}=(p \times p)_{*}\left(p^{*} \tau \times Z \cdot(q \times q)^{*}\left(\iota_{\Delta_{X}}\right)_{*} \sigma\right)=0,
$$


where the second equality holds because $q_{*} p^{*} \tau$ is zero-dimensional and because $\sigma$ has dimension less than $d=\operatorname{dim} X$. Thus $\left(\Gamma_{\sigma}\right)_{*} \tau=0$.

Equation (66) above together with Lemma 11.6 shows that

$$
\left(I^{2}\right)_{*}[x, y]=\alpha[x, y]+(-1)^{d-1} \delta^{d} \cdot I_{*}[x, y] .
$$

Recall from Lemma 10.2 that $I_{*}[x, y]=X_{x}+X_{y}$, and $\delta^{d} \cdot X_{x}=(-1)^{d-1}[x, x]$. It follows that

$$
\left(I^{2}\right)_{*}[x, y]=\alpha[x, y]+[x, x]+[y, y] .
$$

Meanwhile, we can calculate cohomologically the degree of $\left(I^{2}\right)_{*}[x, y]$ as follows. Note that under the Künneth decomposition, the $\mathrm{H}^{0}(X) \otimes \mathrm{H}^{2 d}(X)$-component of $\left[\Delta_{X}\right] \in \mathrm{H}^{2 d}(X)$ is given by

$$
\left[\Delta_{X}\right]_{0,2 d}=[X] \otimes[p t]
$$

By Lemma 11.2, we see that the $\mathrm{H}^{0}(F) \otimes \mathrm{H}^{2 d}(F)$-component of $[I] \in \mathrm{H}^{2 d}(F \times F)$ is given by

$$
[I]_{0,2 d}=(p \times p)_{*}(q \times q)^{*}([X] \otimes[p t])=2[F] \otimes\left[X_{x}\right],
$$

where $x \in X$ is any point. It follows that the $\mathrm{H}^{0}(F) \otimes \mathrm{H}^{4 d}(F)$-component of $I^{2}$ is given by

$$
\left[I^{2}\right]_{0,4 d}=4[F] \otimes\left[X_{x}\right]^{2}=4[F] \otimes[p t] .
$$

Therefore

$$
\operatorname{deg}\left(\left(I^{2}\right)_{*}[x, y]\right)=4
$$

This implies that $\alpha+2=4$. The proof of Proposition 11.4 is complete.

Let us conclude this section with the following proposition.

Proposition 11.7. Let $I \in \mathrm{CH}^{d}(F \times F)$ be the incidence correspondence. Then

$$
\iota_{\Delta}^{*} I=p_{*} q^{*} c_{d}(X)+\sum_{i=1}^{d}(-1)^{i-1} p_{*} q^{*} c_{d-i}(X) \cdot \delta^{i},
$$

where $\iota_{\Delta}: F \rightarrow F \times F$ is the diagonal embedding.

Proof. We take on the notations from the proof of Proposition 11.4. The image $\Gamma_{p}$ of the morphism $(1, p): Z \hookrightarrow Z \times F$ coincides with $(p \times 1)^{-1} \Delta_{F}$. From the commutative diagram

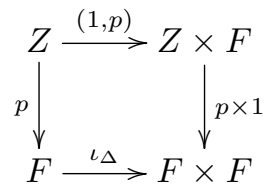

we get

$$
\begin{aligned}
p^{*} \iota_{\Delta}^{*} I & =(1, p)^{*}(p \times 1)^{*} I \\
& =(1, p)^{*}\left(\tilde{I}+\tilde{I}^{\prime}\right) \\
& =c_{d}\left((1, p)^{*} \mathscr{N}_{\tilde{I} / Z \times F}\right)+c_{d}\left((1, p)^{*} \mathscr{N}_{\tilde{I}^{\prime} / Z \times F}\right) \\
& =p^{*} p_{*} c_{d}\left((1, p)^{*} \mathscr{N}_{\tilde{I} / Z \times F}\right) .
\end{aligned}
$$

Note that $\mathscr{N}_{\tilde{I} / Z \times F} \cong\left(q \times\left. 1\right|_{\tilde{I}}\right)^{*} \mathscr{N}_{Z / X \times F}$ and that $\left(q \times\left. 1\right|_{\tilde{I}}\right) \circ(1, p)$ is the identity morphism. Thus $(1, p)^{*} \mathscr{N}_{\tilde{I} / Z \times F} \cong \mathscr{N}_{Z / X \times F}$. It follows that

$$
\iota_{\Delta}^{*} I=p_{*} c_{d}\left(\mathscr{N}_{Z / X \times F}\right) .
$$

By (64), we have

$$
c_{d}\left(\mathscr{N}_{Z / X \times F}\right)=q^{*} c_{d}(X)+\sum_{i=1}^{d}(-1)^{i-1} q^{*} c_{d-i}(X) \cdot E^{i} .
$$


Note that $E^{i}=p^{*} \delta^{i}$. We apply $p_{*}$ to the above equation and get

$$
\iota_{\Delta}^{*} I=p_{*} q^{*} c_{d}(X)+\sum_{i=1}^{d}(-1)^{i-1} p_{*} q^{*} c_{d-i}(X) \cdot \delta^{i} .
$$

This proves the lemma.

\section{Decomposition Results on the Chow groups of $X^{[2]}$}

Let $X$ be a smooth projective variety of dimension $d$ and $F=X^{[2]}$ as in the previous two sections. In that generality, it follows easily from the results in the previous section that the action of $I^{2}=I \cdot I \in$ $\mathrm{CH}^{2 d}(F \times F)$ on $\mathrm{CH}_{0}(F)$ diagonalizes with eigenvalues 2 and 4 ; see Proposition 12.2. When $X$ is a K3 surface or a smooth Calabi-Yau complete intersection, the cycle $\Delta_{\text {tot }}$ introduced in Definition 8.8 is supported on $D \times X \times X$ for some divisor $D \subset X$ (see Remark 12.4), and as a consequence we give other characterizations of the eigenspaces for the action of $\left(I^{2}\right)_{*}$ on $\mathrm{CH}_{0}(F)$; see Propositions 12.7 and 12.8 for the eigenvalue 4, and Proposition 12.9 for the eigenvalue 2.

Along the proof of Proposition 11.4, we have proved the following.

Proposition 12.1. The action of $\left(I^{2}\right)_{*}$ on $\mathrm{CH}_{0}(F)$ can be described as

$$
\left(I^{2}\right)_{*} \sigma=2 \sigma+(-1)^{d-1} \delta^{d} \cdot I_{*} \sigma, \quad \forall \sigma \in \mathrm{CH}_{0}(F) .
$$

In concrete terms, we have

$$
\left(I^{2}\right)_{*}[x, y]=2[x, y]+[x, x]+[y, y] .
$$

We may then deduce the following eigenspace decomposition.

Proposition 12.2. The correspondence $\left(I^{2}-4 \Delta_{F}\right) \circ\left(I^{2}-2 \Delta_{F}\right)$ acts as zero on $\mathrm{CH}_{0}(F)$. Moreover,

$$
\begin{aligned}
\operatorname{ker}\left\{\left(I^{2}-2 \Delta_{F}\right)_{*}: \mathrm{CH}_{0}(F) \rightarrow \mathrm{CH}_{0}(F)\right\} & =\operatorname{ker}\left\{I_{*}: \mathrm{CH}_{0}(F) \rightarrow \mathrm{CH}_{d}(F)\right\} \\
\operatorname{ker}\left\{\left(I^{2}-4 \Delta_{F}\right)_{*}: \mathrm{CH}_{0}(F) \rightarrow \mathrm{CH}_{0}(F)\right\} & =\operatorname{im}\left\{\mathrm{CH}_{0}(\Delta) \rightarrow \mathrm{CH}_{0}(F)\right\} \\
& =\operatorname{im}\left\{\delta^{d} \cdot: \mathrm{CH}_{d}(F) \rightarrow \mathrm{CH}_{0}(F)\right\} .
\end{aligned}
$$

Here, $\Delta$ denotes the sub-variety of $X^{[2]}$ parameterizing non-reduced subschemes of length 2 on $X$.

Proof. That $\left(I^{2}-4 \Delta_{F}\right) \circ\left(I^{2}-2 \Delta_{F}\right)$ acts as zero on $\mathrm{CH}_{0}(F)$ follows immediately from Proposition 12.1 and from the two formulas : $I_{*}[x, y]=X_{x}+X_{y}$ and $\delta^{d} \cdot X_{x}=(-1)^{d-1}[x, x]$ (Lemma 10.2). It is also immediate from Proposition 12.1 that $\operatorname{ker}\left\{I_{*}\right\} \subseteq \operatorname{ker}\left\{\left(I^{2}-2 \Delta_{F}\right)_{*}\right\}$. The inclusion $\operatorname{ker}\left\{I_{*}\right\} \supseteq \operatorname{ker}\left\{\left(I^{2}-2 \Delta_{F}\right)_{*}\right\}$ follows from the following computation

$I_{*}\left(\delta^{d} \cdot I_{*}[x, y]\right)=I_{*}\left(\delta^{d} \cdot\left(X_{x}+X_{y}\right)\right)=(-1)^{d-1} I_{*}([x, x]+[y, y])=(-1)^{d-1} \cdot 2\left(X_{x}+X_{y}\right)=(-1)^{d-1} \cdot 2 I_{*}[x, y]$.

The image of $\mathrm{CH}_{0}(\Delta) \rightarrow \mathrm{CH}_{0}(F)$ is spanned by the classes of the points $[x, x]$. By (67), it is clear that $\left(I^{2}\right)_{*}[x, x]=4[x, x]$. Conversely, if $\sigma \in \mathrm{CH}_{0}(F)$ is such that $\left(I^{2}\right)_{*} \sigma=4 \sigma$, then by Proposition 12.1 we have $2 \sigma=(-1)^{d-1} \delta^{d} \cdot I_{*} \sigma$. Therefore $2 \sigma \in \operatorname{im}\left\{\delta^{d}: \mathrm{CH}_{d}(F) \rightarrow \mathrm{CH}_{0}(F)\right\}$, which concludes the proof since we are working with rational coefficients.

We now wish to turn our focus on K3 surfaces and give other characterizations of the eigenspace decomposition above. As it turns out, the property of K3 surfaces that is going to be used is also satisfied by smooth Calabi-Yau complete intersections. This property can be stated as follows.

Assumption 12.3. There exists a special cycle $\mathfrak{o}_{X} \in \mathrm{CH}_{0}(X)$ of degree 1 such that

$$
p_{1}^{*}\left(x-\mathfrak{o}_{X}\right) \cdot p_{2}^{*}\left(x-\mathfrak{o}_{X}\right)=0
$$

in $\mathrm{CH}_{0}(X \times X)$ for all $x \in X$, where $p_{i}: X \times X \rightarrow X$ are the two projections. 
Remark 12.4. One observes that the cycle $p_{1}^{*}\left(x-\mathfrak{o}_{X}\right) \cdot p_{2}^{*}\left(x-\mathfrak{o}_{X}\right)$ can be obtained by restricting the cycle $\Delta_{\text {tot }}$ of Definition 8.8 to $\{x\} \times X \times X$. Therefore, by Bloch-Srinivas [14], Assumption 12.3 is equivalent to asking for the existence of a special cycle $\mathfrak{o}_{X} \in \mathrm{CH}_{0}(X)$ of degree 1 such that $\Delta_{\text {tot }}$ is supported on $D \times X \times X$ for some divisor $D \subset X$. This is proved for smooth Calabi-Yau hypersufaces by Voisin [55] and for smooth Calabi-Yau complete intersections by Fu [22]. In the case of K3 surfaces, $\Delta_{\text {tot }}=0$ by Theorem 8.10 of Beauville-Voisin. Thus Assumption 12.3 is satisfied by K3 surfaces and smooth Calabi-Yau complete intersections.

Definition 12.5. Given $\mathfrak{o}_{X} \in \mathrm{CH}_{0}(X)$, we define

$$
\mathfrak{o}_{F}:=p_{*} \rho^{*}\left(p_{1}^{*} \mathfrak{o}_{X} \cdot p_{2}^{*} \mathfrak{o}_{X}\right) \in \mathrm{CH}_{0}(F),
$$

where $p_{i}: X \times X \rightarrow X, i=1,2$, are the projections. We also define

$$
X_{\mathfrak{o}}:=p_{*} q^{*} \mathfrak{o}_{X} \in \mathrm{CH}_{d}(F) .
$$

We say that $\mathfrak{o}_{X}$ is effective if it is the class of a single point. Assume that $\mathfrak{o}_{X}$ is effective. By abuse of notation, we will use $\mathfrak{o}_{X}$ to denote both the cycle class and an actual point representing this canonical class. We also use $X_{\mathfrak{o}}$ to denote the variety $p\left(q^{-1} \mathfrak{o}_{X}\right)$. Furthermore, $\mathfrak{o}_{F}$ is also effective since it is represented by the point $\left[\mathfrak{o}_{X}, \mathfrak{o}_{X}\right] \in F$. In this situation where $\mathfrak{o}_{X}$ is supposed to be effective, Assumption 12.3 can be restated as requiring that, for all points $x \in X$, we have

$$
[x, x]-2\left[\mathfrak{o}_{X}, x\right]+\left[\mathfrak{o}_{X}, \mathfrak{o}_{X}\right]=0 \text { in } \mathrm{CH}_{0}(F) .
$$

Let us then state the following proposition.

Proposition 12.6. Let $X$ be a K3 surface or a smooth Calabi-Yau complete intersection. Then there exists a point $\mathfrak{o}_{X} \in X$ such that for all points $x \in X$ we have

$$
[x, x]-2\left[\mathfrak{o}_{X}, x\right]+\left[\mathfrak{o}_{X}, \mathfrak{o}_{X}\right]=0 \text { in } \mathrm{CH}_{0}(F) .
$$

Moreover, the intersection of any two positive-dimensional cycles of complementary codimension inside $X$ is rationally equivalent to a multiple of $\mathfrak{o}_{X}$.

Proof. The first part of the proposition is a combination of the discussion above and Remark 12.4. The second part of the proposition concerned with intersection of cycles is proved by Beauville-Voisin [11] in the case of K3 surfaces, by Voisin [55] in the case of smooth Calabi-Yau hypersurfaces, and by Fu [22] in the case of smooth Calabi-Yau complete intersections. Note that in the case of K3 surfaces, Beauville and Voisin first establish that the intersection of any two divisors on a K3 surface is a multiple of $\mathfrak{o}_{X}$ and then, after some intricate arguments, deduce that $\Delta_{\text {tot }}=0$, whereas in the case of smooth Calabi-Yau complete intersections the logic is reversed : it is first proved that $\Delta_{\text {tot }}$ is supported on a nice divisor and then the intersection property is easily deduced.

We then have the following complement to Proposition 12.2.

Proposition 12.7. If $X$ satisfies Assumption 12.3 with $\mathfrak{o}_{X}$ effective, then

$$
\begin{aligned}
\operatorname{ker}\left\{\left(I^{2}-4 \Delta_{F}\right)_{*}: \mathrm{CH}_{0}(F) \rightarrow \mathrm{CH}_{0}(F)\right\} & =\operatorname{im}\left\{\mathrm{CH}_{0}\left(X_{\mathfrak{o}}\right) \rightarrow \mathrm{CH}_{0}(F)\right\} \\
& =\operatorname{im}\left\{X_{\mathfrak{o}} \cdot: \mathrm{CH}_{d}(F) \rightarrow \mathrm{CH}_{0}(F)\right\} .
\end{aligned}
$$

Proof. The image of $\mathrm{CH}_{0}\left(X_{\mathfrak{o}}\right) \rightarrow \mathrm{CH}_{0}(F)$ is spanned by the cycle classes of points $\left[\mathfrak{o}_{X}, x\right]$. A direct computation using (67) and (70) shows that $\left(I^{2}\right)_{*}\left[\mathfrak{o}_{X}, x\right]=4\left[\mathfrak{o}_{X}, x\right]$. Conversely, Proposition 12.2 shows that $\operatorname{ker}\left\{\left(I^{2}-4 \Delta_{F}\right)_{*}: \mathrm{CH}_{0}(F) \rightarrow \mathrm{CH}_{0}(F)\right\}$ is spanned by cycle classes of points $[x, x]$. But then, (70) says that $[x, x]=2\left[\mathfrak{o}_{X}, x\right]-\left[\mathfrak{o}_{X}, \mathfrak{o}_{X}\right]=X_{\mathfrak{o}} \cdot\left(2 X_{x}-X_{\mathfrak{o}}\right)$, which implies that $\operatorname{ker}\left\{\left(I^{2}-4 \Delta_{F}\right)_{*}: \mathrm{CH}_{0}(F) \rightarrow\right.$ $\left.\mathrm{CH}_{0}(F)\right\} \subseteq \operatorname{im}\left\{X_{\mathfrak{o}} \cdot: \mathrm{CH}_{d}(F) \rightarrow \mathrm{CH}_{0}(F)\right\}$.

Proposition 12.8. Let $X$ be a smooth projective variety with $\mathrm{H}^{1}(X, \mathbb{Z})=0$. Assume that Assumption 12.3 holds on $X$ and that the intersection of any $d$ divisors on $X$ is a multiple of $\mathfrak{o}_{X}$ (e.g., $X$ a $K 3$ surface or a smooth Calabi-Yau complete intersection ; cf. Proposition 12.6), then

$$
\mathrm{CH}^{1}(F)^{\cdot d} \cdot I_{*} \mathrm{CH}_{0}(F)=\operatorname{ker}\left\{\left(I^{2}-4 \Delta_{F}\right)_{*}: \mathrm{CH}_{0}(F) \rightarrow \mathrm{CH}_{0}(F)\right\} .
$$


Proof. By Proposition 12.2, $\operatorname{ker}\left\{\left(I^{2}-4 \Delta_{F}\right)_{*}\right\}$ is spanned by the classes of the points $[x, x]$ for all $x \in X$. Since $[x, x]=(-1)^{d-1} \delta^{d} \cdot I_{*} x$, we get the inclusion " $\supseteq$ ".

As for the inclusion " $\subseteq$ ", it is enough to show, thanks to Propositions 12.2 and 12.7 , that $D^{d} \cdot X_{x}$ is a linear combination of $[x, x]$ and $[x, \mathfrak{o}]$ for all divisors $D$ and all points $x \in X$. The decomposition (55) implies that any divisor $D$ on $F$ is of the form

$$
D=\hat{\mathfrak{D}}+a \delta, \text { where } \hat{\mathfrak{D}}=p_{*} q^{*} \mathfrak{D}, \mathfrak{D} \in \mathrm{CH}^{1}(X) \text {, and } a \in \mathbb{Q} .
$$

Given $\sigma \in \mathrm{CH}_{1}(F), \delta \cdot \sigma$ belongs to the image of $\mathrm{CH}_{0}(\Delta) \rightarrow \mathrm{CH}_{0}(F)$, so that by Proposition 12.2 it suffices to verify :

$$
\hat{\mathfrak{D}}^{d} \cdot X_{x} \text { is a linear combination of }[x, x] \text { and }[x, \mathfrak{o}] .
$$

Recall that we have the following diagram

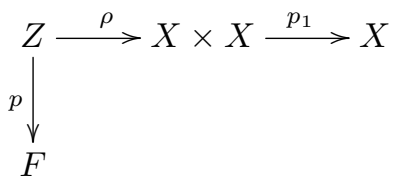

with $q=p_{1} \circ \rho$. Let us compute $\hat{\mathfrak{D}}^{d}$ for any divisor $\hat{\mathfrak{D}}$ of the form $p_{*} q^{*} \mathfrak{D}, \mathfrak{D} \in \mathrm{CH}^{1}(X)$.

$$
\begin{aligned}
\hat{\mathfrak{D}}^{d} & =p_{*} q^{*} \mathfrak{D} \cdot\left(p_{*} q^{*} \mathfrak{D}\right)^{d-1} \\
& =p_{*}\left(q^{*} \mathfrak{D} \cdot\left(p^{*} p_{*} q^{*} \mathfrak{D}\right)^{d-1}\right) \\
& =p_{*}\left(\rho^{*} p_{1}^{*} \mathfrak{D} \cdot\left(\rho^{*} p_{1}^{*} \mathfrak{D}+\rho^{*} p_{2}^{*} \mathfrak{D}\right)^{d-1}\right) \\
& =p_{*} \rho^{*} p_{1}^{*}\left(\mathfrak{D}^{d}\right)+\sum_{i=1}^{d-1} c_{i} p_{*} \rho^{*}\left(p_{1}^{*} \mathfrak{D}^{d-i} \cdot p_{2}^{*} \mathfrak{D}^{i}\right), \quad c_{i} \in \mathbb{Q} \\
& =\operatorname{deg}\left(\mathfrak{D}^{d}\right) X_{\mathfrak{o}}+\sum_{i=1}^{d-1} c_{i} p_{*} \rho^{*}\left(\mathfrak{D}^{d-i} \times \mathfrak{D}^{i}\right) .
\end{aligned}
$$

where the last equality holds since $\mathfrak{D}^{d}$ is a multiple of $\mathfrak{o}_{X}$. Thus

$$
\hat{\mathfrak{D}}^{d} \cdot X_{x}=\operatorname{deg}\left(\mathfrak{D}^{d}\right)\left[x, \mathfrak{o}_{X}\right] .
$$

Actually, the above can be made more precise. We have

$$
\delta \cdot \hat{\mathfrak{D}} \cdot X_{x}=0, \quad \text { for all } x \in X .
$$

Indeed, $\delta \cdot \hat{\mathfrak{D}}$ can be represented by a divisor $E$ on $\Delta$, so that for general $x \in X, S_{x}$ does not meet $E$. Thus we have proved

$$
D^{d} \cdot X_{x}=(-1)^{d-1} a^{d}[x, x]+\operatorname{deg}\left(\mathfrak{D}^{d}\right)\left[x, \mathfrak{o}_{X}\right] .
$$

Since $X_{x} \cdot X_{y}=[x, y]$, it is clear that the intersection product

$$
\mathrm{CH}_{d}(F) \otimes \mathrm{CH}_{d}(F) \rightarrow \mathrm{CH}_{0}(F)
$$

is surjective. We define

$$
\mathcal{A}:=I_{*} \mathrm{CH}_{0}(F) \subseteq \mathrm{CH}_{d}(F) .
$$

The following proposition essentially shows, as will become apparent once $L$ is defined, the equality $\mathrm{CH}^{2}(F)_{2} \cdot \mathrm{CH}^{2}(F)_{2}=\mathrm{CH}^{4}(F)_{4}$ of Theorem 3 in the case when $F$ is the Hilbert scheme of length-2 subschemes on a K3 surface.

Proposition 12.9. Let $X$ be a smooth projective variety. We have the following inclusions

$$
\mathcal{A}_{\text {hom }} \cdot \mathcal{A}_{\text {hom }} \subseteq \operatorname{ker}\left\{Z_{*}: \mathrm{CH}_{0}(F) \rightarrow \mathrm{CH}_{0}(X)\right\} \subseteq \operatorname{ker}\left\{I_{*}: \mathrm{CH}_{0}(F) \rightarrow \mathrm{CH}_{d}(F)\right\} ;
$$

and $\mathcal{A}_{\text {hom }} \cdot \mathcal{A}_{\text {hom }}$ is spanned by cycles of the form $[x, y]+[z, w]-[x, z]-[y, w]$. Moreover, if $X$ satisfies Assumption 12.3, then the above inclusions are all equalities. 
Proof. Two distinct points $x, y \in X$ determine a point $[x, y] \in F$ and a general point of $F$ is always of this form. By definition we have

$$
I_{*}[x, y]=X_{x}+X_{y}=Z^{*} Z_{*}[x, y] .
$$

If $\sigma \in \mathrm{CH}_{0}(F)$ is such that $Z_{*} \sigma=0$, then by the above equality we have $I_{*} \sigma=0$. Thus $\operatorname{ker}\left(Z_{*}\right) \subseteq \operatorname{ker}\left(I_{*}\right)$. Recall that $X_{x} \cdot X_{y}=[x, y]$ so that, for a general point $p \in X$, we have

$$
\begin{aligned}
{[x, y]+[z, w]-[x, z]-[y, w] } & =\left(X_{x}-X_{w}\right) \cdot\left(X_{y}-X_{z}\right) \\
& =I_{*}([x, p]-[w, p]) \cdot I_{*}([y, p]-[z, p]),
\end{aligned}
$$

which by bilinearity shows that $\mathcal{A}_{\text {hom }} \cdot \mathcal{A}_{\text {hom }}$ is spanned by cycles of the form $[x, y]+[z, w]-[x, z]-[y, w]$. It is then immediate to see that $\mathcal{A}_{\text {hom }} \cdot \mathcal{A}_{\text {hom }} \subseteq \operatorname{ker}\left\{Z_{*}\right\}$.

Let us now assume that $X$ satisfies Assumption 12.3 and let us show that $\operatorname{ker}\left\{I_{*}\right\} \subseteq \mathcal{A}_{\text {hom }} \cdot \mathcal{A}_{\text {hom }}$. We further assume that $\mathfrak{o}_{X}$ is effective. Otherwise, in what follows, we replace $\left[x, \mathfrak{o}_{X}\right]$ by $p_{*} \rho^{*}\left(p_{1}^{*} x \cdot p_{2}^{*} \mathfrak{o}_{X}\right)$ and $\left[\mathfrak{o}_{X}, \mathfrak{o}_{X}\right]$ by $p_{*} \rho^{*}\left(p_{1}^{*} \mathfrak{o}_{X} \cdot p_{2}^{*} \mathfrak{o}_{X}\right)$. Then the same proof carries through. By Assumption 12.3, the key identity (70)

$$
[x, x]-2\left[x, \mathfrak{o}_{X}\right]+\mathfrak{o}_{F}=0, \quad \text { in } \mathrm{CH}_{0}(F)
$$

is satisfied. Now let $\sigma=\sum_{i=1}^{r}\left[x_{i}, y_{i}\right]-\sum_{i=1}^{r}\left[z_{i}, w_{i}\right] \in \mathrm{CH}_{0}(F)_{\text {hom }}$ be such that $I_{*} \sigma=0$, i.e.,

$$
\sum\left(X_{x_{i}}+X_{y_{i}}\right)-\sum\left(X_{z_{i}}+X_{w_{i}}\right)=0, \quad \text { in } \mathrm{CH}_{d}(F) .
$$

By intersecting the above sum with $X_{\mathfrak{o}_{X}}$, we get

$$
\sum\left(\left[x_{i}, \mathfrak{o}_{X}\right]+\left[y_{i}, \mathfrak{o}_{X}\right]\right)-\sum\left(\left[z_{i}, \mathfrak{o}_{X}\right]+\left[w_{i}, \mathfrak{o}_{X}\right]\right)=0, \quad \text { in } \mathrm{CH}_{0}(F) .
$$

Combined with equation (70), this yields $\sum\left(\left[x_{i}, x_{i}\right]+\left[y_{i}, y_{i}\right]-\left[z_{i}, z_{i}\right]-\left[w_{i}, w_{i}\right]\right)=0$. Hence it follows that

$$
\begin{aligned}
2 \sigma & =2\left(\sum\left[x_{i}, y_{i}\right]-\sum\left[z_{i}, w_{i}\right]\right) \\
& =\sum\left(X_{x_{i}}-X_{y_{i}}\right)^{2}-\sum\left(X_{z_{i}}-X_{w_{i}}\right)^{2}-\sum\left(\left[x_{i}, x_{i}\right]+\left[y_{i}, y_{i}\right]-\left[z_{i}, z_{i}\right]-\left[w_{i}, w_{i}\right]\right) \\
& =\sum\left(X_{x_{i}}-X_{y_{i}}\right)^{2}-\sum\left(X_{z_{i}}-X_{w_{i}}\right)^{2} \\
& =\sum\left(I_{*}\left(\left[x_{i}, \mathfrak{o}_{X}\right]-\left[y_{i}, \mathfrak{o}_{X}\right]\right)\right)^{2}-\sum\left(I_{*}\left(\left[z_{i}, \mathfrak{o}_{X}\right]-\left[w_{i}, \mathfrak{o}_{X}\right]\right)\right)^{2} .
\end{aligned}
$$

This shows that $\operatorname{ker}\left\{I_{*}\right\} \subseteq \mathcal{A}_{\text {hom }} \cdot \mathcal{A}_{\text {hom }}$.

\section{Multiplicative Chow-KünNeth Decomposition for $X^{[2]}$}

In this section, we consider a smooth projective variety $X$ of dimension $d$ endowed with a multiplicative Chow-Künneth decomposition in the sense of Definition 8.1. Recall that, denoting $\Delta_{123}^{X}$ the class of $\{(x, x, x): x \in X\}$ inside $X \times X \times X$ seen as a correspondence of degree $d$ from $X \times X$ to $X$, this means that there is a Chow-Künneth decomposition of the diagonal

$$
\Delta_{X}=\sum_{i=0}^{2 d} \pi_{X}^{i}, \quad \text { such that } \Delta_{123}^{X}=\sum_{i+j=k} \pi_{X}^{k} \circ \Delta_{123}^{X} \circ\left(\pi_{X}^{i} \otimes \pi_{X}^{j}\right) .
$$

In particular, such a multiplicative decomposition induces a bigrading of the Chow ring

$$
\mathrm{CH}^{*}(X)=\bigoplus_{p, s} \mathrm{CH}_{\mathrm{CK}}^{p}(X)_{s}, \quad \text { with } \quad \mathrm{CH}_{\mathrm{CK}}^{p}(X)_{s}:=\left(\pi_{X}^{2 p-s}\right)_{*} \mathrm{CH}^{p}(X) .
$$

Under the condition that the Chern classes of the tangent bundle of $X$ belong to the degree-zero graded part of the above decomposition, and viewing $X^{[2]}$ as a quotient of the blow-up of $X \times X$ along the diagonal for the action of the symmetric group $\mathfrak{S}_{2}$, we show in Theorem 13.4 that $X^{[2]}$ is naturally endowed with a multiplicative Chow-Künneth decomposition. We first show that the projective normal bundle of $X$ can be endowed with a multiplicative Chow-Künneth decomposition. Then we show that the blow-up of $X$ along the diagonal has a multiplicative $\mathfrak{S}_{2}$-equivariant Chow-Künneth decomposition. 
Finally we deduce that $X^{[2]}$ has a multiplicative Chow-Künneth decomposition. The point of Section 15 will be to check that the decomposition induced by this multiplicative Chow-Künneth decomposition on the Chow groups of $S^{[2]}$ coincides with the decomposition induced by the Fourier transform. Finally, in $\S 13.4$, we prove Theorem 6 .

13.1. Multiplicative Chow-Künneth decompositions for projective bundles. Let $\mathscr{E}$ be a locally free coherent sheaf of rank $r+1$ and let $\pi: \mathbb{P}(\mathscr{E}) \rightarrow X$ be the associated projective bundle. Let $\xi \in \mathrm{CH}^{1}(\mathbb{P}(\mathscr{E}))$ be the class of the relative $\mathcal{O}(1)$-bundle. Then the Chow ring of $\mathbb{P}(\mathscr{E})$ is given by

$$
\mathrm{CH}^{*}(\mathbb{P}(\mathscr{E}))=\mathrm{CH}^{*}(X)[\xi], \quad \text { where } \quad \xi^{r+1}+\pi^{*} c_{1}(\mathscr{E}) \xi^{r}+\cdots+\pi^{*} c_{r}(\mathscr{E}) \xi+\pi^{*} c_{r+1}(\mathscr{E})=0 .
$$

The same formula holds for the cohomology ring of $\mathbb{P}(\mathscr{E})$. We may then write, for each $i \geq 0$,

$$
\xi^{i}=\sum_{j=0}^{r} \pi^{*} a_{i, j} \xi^{j},
$$

for some unique $a_{i, j} \in \mathrm{CH}^{i-j}(X)$, where $a_{i, j}$ is a polynomial of the Chern classes of $\mathscr{E}$. It follows in particular that $\pi_{*} \xi^{i}=a_{i, r}$ is a polynomial of the Chern classes of $\mathscr{E}$.

Proposition 13.1. Suppose $X$ has a multiplicative Chow-Künneth decomposition and $c_{i}(\mathscr{E}) \in \mathrm{CH}_{\mathrm{CK}}^{i}(X)_{0}$ for all $i \geq 0$. Then $\mathbb{P}(\mathscr{E})$ has a multiplicative Chow-Künneth decomposition such that the associated decomposition of the Chow ring of $\mathbb{P}(\mathscr{E})$ satisfies

$$
\mathrm{CH}_{\mathrm{CK}}^{p}(\mathbb{P}(\mathscr{E}))_{s}=\bigoplus_{l=0}^{r} \xi^{l} \cdot \pi^{*} \mathrm{CH}_{\mathrm{CK}}^{p-l}(X)_{s} .
$$

Proof. The projective bundle formula for Chow groups gives an isomorphism

$$
\sum_{l=0}^{r} \xi^{l} \cdot \pi^{*}: \bigoplus_{l=0}^{r} \mathrm{CH}^{p-l}(X) \stackrel{\simeq}{\longrightarrow} \mathrm{CH}^{p}(\mathbb{P}(\mathscr{E})) .
$$

This isomorphism is in fact an isomorphism of motives ; see Manin [34]. Therefore, the Chow-Künneth decomposition of $X$ induces a Chow-Künneth decomposition of $\Delta_{\mathbb{P}(\mathscr{E})}$ whose associated pieces $\mathrm{CH}_{\mathrm{CK}}^{p}(\mathbb{P}(\mathscr{E}))_{s}$ of the Chow groups $\mathrm{CH}^{p}(X)$ are described in the formula (76).

The self-product $\mathbb{P}(\mathscr{E}) \times \mathbb{P}(\mathscr{E}) \times \mathbb{P}(\mathscr{E})$ can be obtained from $X \times X \times X$ by successively taking the projectivizations of the vector bundles $p_{i}^{*} \mathscr{E}, i=1,2,3$. The subtle point here is that there are thus two ways of obtaining a Chow-Künneth decomposition for $\mathbb{P}(\mathscr{E}) \times \mathbb{P}(\mathscr{E}) \times \mathbb{P}(\mathscr{E})$ : either one considers the product Chow-Künneth decomposition on $\mathbb{P}(\mathscr{E}) \times \mathbb{P}(\mathscr{E}) \times \mathbb{P}(\mathscr{E})$ induced by that of $\mathbb{P}(\mathscr{E})$ (and this is the one that matters in order to establish the multiplicativity property), or one considers the product Chow-Künneth decomposition on $X \times X \times X$ induced by that of $X$ and then uses the projective bundle isomorphism together with Manin's identity principle to produce a Chow-Künneth decomposition on the successive projectivizations of the vector bundles $p_{i}^{*} \mathscr{E}, i=1,2,3$ (and this is the one used to compute the induced decomposition on the Chow groups of $\mathbb{P}(\mathscr{E}) \times \mathbb{P}(\mathscr{E}) \times \mathbb{P}(\mathscr{E})$ in terms of the Chow groups of $X \times X \times X$ as in (77) below). Either way, these Chow-Künneth decompositions agree. In particular, the decompositions induced on the Chow groups of $\mathbb{P}(\mathscr{E}) \times \mathbb{P}(\mathscr{E}) \times \mathbb{P}(\mathscr{E})$ agree and satisfy :

$$
\mathrm{CH}_{\mathrm{CK}}^{p}(\mathbb{P}(\mathscr{E}) \times \mathbb{P}(\mathscr{E}) \times \mathbb{P}(\mathscr{E}))_{s}=\bigoplus_{0 \leq i, j, k \leq r}\left(\pi^{\times 3}\right)^{*} \mathrm{CH}_{\mathrm{CK}}^{p-i-j-k}(X \times X \times X)_{s} \cdot \xi_{1}^{i} \xi_{2}^{j} \xi_{3}^{k},
$$

where $\pi^{\times 3}: \mathbb{P}(\mathscr{E}) \times \mathbb{P}(\mathscr{E}) \times \mathbb{P}(\mathscr{E}) \rightarrow X \times X \times X$ is the the 3 -fold product of $\pi$, and where $\xi_{i} \in$ $\mathrm{CH}^{1}(\mathbb{P}(\mathscr{E}) \times \mathbb{P}(\mathscr{E}) \times \mathbb{P}(\mathscr{E}))$ is the pull-back of $\xi \in \mathrm{CH}^{1}(\mathbb{P}(\mathscr{E}))$ from the $i^{\text {th }}$ factor, $i=1,2,3$.

By the criterion for multiplicativity of a Chow-Künneth decomposition given in Proposition 8.4, we only need to show that

$$
\Delta_{123}^{\mathrm{P}(\mathscr{E})} \in \mathrm{CH}_{\mathrm{CK}}^{2 d+2 r}(\mathrm{P}(\mathscr{E}) \times \mathbb{P}(\mathscr{E}) \times \mathbb{P}(\mathscr{E}))_{0}
$$


where $\Delta_{123}^{\mathrm{P}(\mathscr{E})}$ is the small diagonal of $\mathbb{P}(\mathscr{E})$ (cf. Definition 8.8). Consider the following diagram

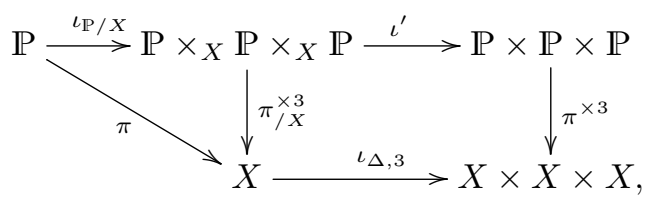

where in order to lighten the notations we have set $\mathbb{P}:=\mathbb{P}(\mathscr{E})$, and where $\iota_{\mathbb{P} / X}$ is the small diagonal embedding of $\mathbb{P}$ relative to $X$. By definition, we have

$$
\Delta_{123}^{\mathrm{P}(\mathscr{E})}=\left(\iota^{\prime}\right)_{*}\left(\iota_{\mathbb{P} / X}\right)_{*}[\mathbb{P}] .
$$

Note that the Chow groups of $\mathbb{P} \times_{X} \mathbb{P} \times_{X} \mathbb{P}$ are given by

$$
\mathrm{CH}^{p}\left(\mathbb{P} \times_{X} \mathbb{P} \times_{X} \mathbb{P}\right)=\bigoplus_{0 \leq i, j, k \leq r}\left(\pi_{/ X}^{\times 3}\right)^{*} \mathrm{CH}^{p-i-j-k}(X) \cdot\left(\xi_{1}^{\prime}\right)^{i}\left(\xi_{2}^{\prime}\right)^{j}\left(\xi_{3}^{\prime}\right)^{k},
$$

where $\xi_{i}^{\prime}=\left(\iota^{\prime}\right)^{*} \xi_{i}$. Hence we have

$$
\left(\iota_{\mathbb{P} / X}\right)_{*}[\mathbb{P}]=\sum_{0 \leq i, j, k \leq r}\left(\pi_{/ X}^{\times 3}\right)^{*} \alpha_{i j k} \cdot\left(\xi_{1}^{\prime}\right)^{i}\left(\xi_{2}^{\prime}\right)^{j}\left(\xi_{3}^{\prime}\right)^{k}
$$

for some $\alpha_{i j k} \in \mathrm{CH}^{*}(X)$. For each triple $\left(l_{1}, l_{2}, l_{3}\right)$ of non-negative integers, we intersect the above equation with $\left(\xi_{1}^{\prime}\right)^{l_{1}}\left(\xi_{2}^{\prime}\right)^{l_{2}}\left(\xi_{3}^{\prime}\right)^{l_{3}}$ and then push forward to $X$ along $\pi_{/ X}^{\times 3}$. Noting that

$$
\left(\pi_{/ X}^{\times 3}\right)_{*}\left(\left(\iota_{\mathbb{P} / X}\right)_{*}[\mathbb{P}] \cdot\left(\xi_{1}^{\prime}\right)^{l_{1}}\left(\xi_{2}^{\prime}\right)^{l_{2}}\left(\xi_{3}^{\prime}\right)^{l_{3}}\right)=\pi_{*} \xi^{l_{1}+l_{2}+l_{3}}=\text { polynomial in }\left\{c_{i}(\mathscr{E})\right\},
$$

we obtain a bunch of linear equations involving the $\alpha_{i j k}$ 's whose coefficients are all polynomials in terms of the Chern classes of $\mathscr{E}$. One inductively deduces from these equations that the $\alpha_{i j k}$ are all polynomials of the Chern classes of $\mathscr{E}$. Then, by assumption, we have $\alpha_{i j k} \in \mathrm{CH}_{\mathrm{CK}}^{*}(X)_{0}$. Note that

$$
\begin{aligned}
\Delta_{123}^{\mathbb{P}(\mathscr{E})} & =\iota_{*}^{\prime}\left(\iota_{\mathbb{P} / X}\right)_{*}[\mathbb{P}] \\
& =\sum_{0 \leq i, j, k \leq r} \iota_{*}^{\prime}\left(\left(\pi_{/ X}^{\times 3}\right)^{*} \alpha_{i j k} \cdot\left(\xi_{1}^{\prime}\right)^{i}\left(\xi_{2}^{\prime}\right)^{j}\left(\xi_{3}^{\prime}\right)^{k}\right) \\
& =\sum_{0 \leq i, j, k \leq r} \iota_{*}^{\prime}\left(\left(\pi_{/ X}^{\times 3}\right)^{*} \alpha_{i j k} \cdot\left(\iota^{\prime}\right)^{*}\left(\xi_{1}^{i} \xi_{2}^{j} \xi_{3}^{k}\right)\right) \\
& =\sum_{0 \leq i, j, k \leq r}\left(\iota_{*}^{\prime}\left(\pi_{/ X}^{\times 3}\right)^{*} \alpha_{i j k}\right) \cdot \xi_{1}^{i} \xi_{2}^{j} \xi_{3}^{k} \\
& =\sum_{0 \leq i, j, k \leq r}\left(\left(\pi^{\times 3}\right)^{*}\left(\iota_{\Delta, 3}\right)_{*} \alpha_{i j k}\right) \cdot \xi_{1}^{i} \xi_{2}^{j} \xi_{3}^{k} .
\end{aligned}
$$

Since $\left(\iota_{\Delta, 3}\right)_{*} \alpha_{i j k} \in \mathrm{CH}_{\mathrm{CK}}^{*}\left(X^{3}\right)_{0}$ by Proposition 8.7, we conclude from (77) that $\Delta_{123}^{\mathrm{P}} \in \mathrm{CH}_{\mathrm{CK}}^{*}(\mathbb{P} \times \mathbb{P} \times$ $\mathbb{P})_{0}$.

13.2. Multiplicative Chow-Künneth decompositions for smooth blow-ups. In this paragraph, we investigate the stability of the multiplicativity property for Chow-Künneth decompositions under smooth blow-up. Let $X$ be a smooth projective variety and let $i: Y \hookrightarrow X$ be a smooth closed sub-variety of codimension $r+1$. Let $\rho: \widetilde{X} \rightarrow X$ be the blow-up of $X$ along $Y$. Consider the following diagram

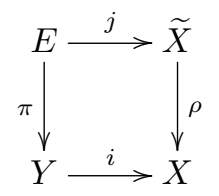

where $E$ is the exceptional divisor on $\widetilde{X}$; it is isomorphic to the geometric projectivization $\mathbb{P}\left(\mathscr{N}_{Y / X}\right)$ of the normal bundle of $Y$ inside $X$. Let $\xi \in \mathrm{CH}^{1}(E)$ be the class of the relative $\mathcal{O}(1)$-bundle of the 
projective bundle $E \rightarrow X$. The smooth blow-up formula for Chow groups [25, Proposition 6.7 (e)] gives an isomorphism

$$
\mathrm{CH}^{p}(X) \oplus \bigoplus_{l=0}^{r-1} \mathrm{CH}^{p-l-1}(Y) \stackrel{\simeq}{\longrightarrow} \mathrm{CH}^{p}(\widetilde{X}), \quad\left(\alpha, \beta_{0}, \ldots, \beta_{r-1}\right) \mapsto \rho^{*} \alpha+\sum_{l=0}^{r-1} j_{*}\left(\xi^{l} \cdot \pi^{*} \beta_{l}\right) .
$$

Proposition 13.2. Assume the following conditions :

(i) both $X$ and $Y$ have a multiplicative Chow-Künneth decomposition;

(ii) $i^{*} \mathrm{CH}_{\mathrm{CK}}^{p}(X)_{s} \subseteq \mathrm{CH}_{\mathrm{CK}}^{p}(Y)_{s}$ and $i_{*} \mathrm{CH}_{\mathrm{CK}}^{p-1}(Y)_{s} \subseteq \mathrm{CH}_{\mathrm{CK}}^{p+r}(X)_{s}$ for all $p$ and $s$;

(iii) the homomorphism $i_{*}: \mathrm{CH}_{*}(Y) \rightarrow \mathrm{CH}_{*}(X)$ is injective;

(iv) $c_{p}\left(\mathscr{N}_{Y / X}\right) \in \mathrm{CH}_{\mathrm{CK}}^{p}(Y)_{0}$ for all $p \geq 0$.

Then $\widetilde{X}$ has a multiplicative Chow-Künneth decomposition such that

$$
\mathrm{CH}_{\mathrm{CK}}^{p}(\widetilde{X})_{s}=\rho^{*} \mathrm{CH}_{\mathrm{CK}}^{p}(X)_{s} \oplus\left(\bigoplus_{l=0}^{r-1} j_{*}\left(\xi^{l} \cdot \pi^{*} \mathrm{CH}_{\mathrm{CK}}^{p-l-1}(Y)_{s}\right)\right) .
$$

Furthermore, if $\mathrm{CH}_{\mathrm{CK}}^{p}(E)_{s}$ is the decomposition obtained in Proposition 13.1, then we have

$$
j^{*} \mathrm{CH}_{\mathrm{CK}}^{p}(\widetilde{X})_{s} \subseteq \mathrm{CH}_{\mathrm{CK}}^{p}(E)_{s} \quad \text { and } \quad j_{*} \mathrm{CH}_{\mathrm{CK}}^{p-1}(E)_{s} \subseteq \mathrm{CH}_{\mathrm{CK}}^{p}(\widetilde{X})_{s} .
$$

Proof. By Manin [34], the isomorphism (79) is in fact an isomorphism of motives so that the ChowKünneth decompositions of $X$ and $Y$ induce a Chow-Künneth decomposition of $\widetilde{X}$ such that the associated pieces of the decomposition of the Chow groups are as given in (80). Before proving that the above decomposition is multiplicative, we first establish the compatibility results for $j^{*}$ and $j_{*}$. We first note that if $\alpha=\rho^{*} \alpha^{\prime}$, for some $\alpha^{\prime} \in \mathrm{CH}_{\mathrm{CK}}^{p}(X)_{s}$, then $i^{*} \alpha^{\prime} \in \mathrm{CH}_{\mathrm{CK}}^{p}(Y)_{s}$ and hence

$$
j^{*} \alpha=\pi^{*} i^{*} \alpha^{\prime} \in \mathrm{CH}_{\mathrm{CK}}^{p}(E)_{s} .
$$

Similarly, if $\alpha=j_{*}\left(\xi^{l} \cdot \pi^{*} \alpha^{\prime}\right)$ for some $\alpha^{\prime} \in \mathrm{CH}_{\mathrm{CK}}^{p-l-1}(Y)_{s}$, then

$$
j^{*} \alpha=j^{*} j_{*}\left(\xi^{l} \cdot \pi^{*} \alpha^{\prime}\right)=\left.E\right|_{E} \cdot \xi^{l} \cdot \pi^{*} \alpha^{\prime}=-\xi^{l+1} \cdot \pi^{*} \alpha^{\prime} \in \mathrm{CH}_{\mathrm{CK}}^{p}(E)_{s},
$$

where we used the fact that $j^{*} E=-\xi$. Hence we have

$$
j^{*} \mathrm{CH}_{\mathrm{CK}}^{p}(\widetilde{X})_{s} \subseteq \mathrm{CH}_{\mathrm{CK}}^{p}(E)_{s} .
$$

To prove the compatibility for $j_{*}$, consider $\alpha=\xi^{l} \cdot \pi^{*} \alpha^{\prime}$ for some $\alpha^{\prime} \in \mathrm{CH}_{\mathrm{CK}}^{p-l}(Y)_{s}$. If $l<r$, then that $j_{*} \alpha$ belongs to $\mathrm{CH}_{\mathrm{CK}}^{p+1}(\tilde{X})_{s}$ is automatic. Assume that $l=r$. Then the key formula of [25, Proposition 6.7 (a)] (which is recalled later on in Lemma 14.1) implies

$$
\begin{aligned}
\rho^{*} i_{*} \alpha^{\prime} & =j_{*}\left(c_{r}\left(\pi^{*} \mathscr{N}_{Y / X} / \mathcal{O}(-1)\right) \cdot \pi^{*} \alpha^{\prime}\right) \\
& =j_{*}\left(\left(\pi^{*} c_{r}\left(\mathscr{N}_{Y / X}\right)+\xi \cdot \pi^{*} c_{r-1}\left(\mathscr{N}_{Y / X}\right)+\cdots+\xi^{r-1} \pi^{*} c_{1}\left(\mathscr{N}_{Y / X}\right)+\xi^{r}\right) \cdot \pi^{*} \alpha^{\prime}\right) .
\end{aligned}
$$

Note that

$$
j_{*}\left(\xi^{l^{\prime}} \cdot \pi^{*}\left(c_{r-l}\left(\mathscr{N}_{Y / X}\right) \cdot \alpha^{\prime}\right)\right) \in \mathrm{CH}_{\mathrm{CK}}^{p+1}(\widetilde{X})_{s},
$$

for all $l^{\prime}<r$. Meanwhile, using the assumption (ii), we also know that $\rho^{*} i_{*} \alpha^{\prime} \in \mathrm{CH}_{\mathrm{CK}}^{p+1}(\widetilde{X})_{s}$. Then one easily sees that $j_{*}\left(\xi^{r} \pi^{*} \alpha^{\prime}\right) \in \mathrm{CH}_{\mathrm{CK}}^{p+1}(\widetilde{X})_{s}$. In all cases, we have $j_{*} \mathrm{CH}_{\mathrm{CK}}^{p}(E)_{s} \subseteq \mathrm{CH}_{\mathrm{CK}}^{p+1}(\widetilde{X})_{s}$.

It remains to show that the Chow-Künneth decomposition of $\tilde{X}$ is multiplicative. As in the proof of Proposition 13.1, we would like to use the criterion of Proposition 8.4 to show that the small diagonal $\Delta_{123}^{\widetilde{X}}$ (cf. Definition 8.8) belongs to $\mathrm{CH}_{\mathrm{CK}}^{2 d}(\widetilde{X} \times \widetilde{X} \times \widetilde{X})_{0}$, where the Chow-Künneth decomposition used is the 3 -fold product Chow-Künneth decomposition on $\widetilde{X}$. For that purpose, we need to understand at least how the Chow groups of $X \times X \times X$ fit into the Chow groups of $\widetilde{X} \times \widetilde{X} \times \widetilde{X}$ (see (82)). Note that the 3 -fold product $\widetilde{X}^{3}$ of $\widetilde{X}$ can also be viewed as blowing up $X \times X \times X$ successively three times into $\widetilde{X} \times X \times X, \widetilde{X} \times \widetilde{X} \times X$ and then into $\widetilde{X} \times \widetilde{X} \times \widetilde{X}$. The crucial point is then to observe that the product Chow-Künneth decomposition on $\widetilde{X}^{3}$ agrees with the one obtained by starting with the product Chow-Künneth decomposition on $X^{3}$ and then by using the blow-up formula (79) and Manin's identity 
principle after each blow-up necessary to obtain $\widetilde{X}^{3}$. As such, these Chow-Künneth decompositions for $\widetilde{X}^{3}$ induce the same decomposition of the Chow groups of $\widetilde{X}^{3}$; in view of (80) applied to $X \times X \times X$, $\widetilde{X} \times X \times X$ and $\widetilde{X} \times \widetilde{X} \times X$, successively, one finds that the induced decomposition on the Chow groups of $\widetilde{X} \times \widetilde{X} \times \widetilde{X}$ satisfies

$$
\left(\rho^{\times 3}\right)^{*} \mathrm{CH}_{\mathrm{CK}}^{p}\left(X^{3}\right)_{s}+\left(j^{\times 3}\right)_{*}\left(\bigoplus_{i_{1}, i_{2}, i_{3}=0}^{r} \xi_{1}^{i_{1}} \xi_{2}^{i_{2}} \xi_{3}^{i_{3}} \cdot\left(\pi^{\times 3}\right)^{*} \mathrm{CH}_{\mathrm{CK}}^{p-3-i_{1}-i_{2}-i_{3}}\left(Y^{3}\right)_{s}\right) \subseteq \mathrm{CH}_{\mathrm{CK}}^{p}\left(\widetilde{X}^{3}\right)_{s} .
$$

As already mentioned, in order to establish the multiplicativity property of the Chow-Künneth decomposition on $\widetilde{X}$, it suffices by the criterion of Proposition 8.4 to show that the small diagonal $\Delta_{123}^{\widetilde{X}}$ satisfies $\Delta_{123}^{\widetilde{X}} \in \mathrm{CH}_{\mathrm{CK}}^{2 d}\left(\widetilde{X}^{3}\right)_{0}$.

Consider the following fiber square

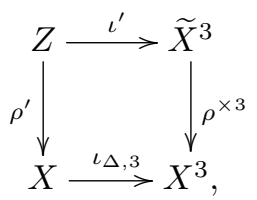

where $Z$ contains two components : $Z_{1} \cong \widetilde{X}$ which is such that the restriction of $\iota^{\prime}$ to $Z_{1}$ is simply the small diagonal embedding $\tilde{\iota}_{\Delta, 3}: \widetilde{X} \rightarrow \widetilde{X}^{3}$, and $Z_{2}=E \times_{Y} E \times_{Y} E$. It follows that

$$
\Delta_{123}^{\widetilde{X}}=\left(\rho^{\times 3}\right)^{*} \Delta_{123}^{X}+\iota_{*}^{\prime} \alpha,
$$

for some $\alpha \in \mathrm{CH}^{*}\left(E \times_{Y} E \times_{Y} E\right)$. By equation (78), we get

$$
\alpha=\sum_{i_{1}, i_{2}, i_{3}=0}^{r}\left(\pi_{/ Y}^{\times 3}\right)^{*} \alpha_{i_{1} i_{2} i_{3}} \cdot\left(\xi_{1}^{\prime}\right)^{i_{1}}\left(\xi_{2}^{\prime}\right)^{i_{2}}\left(\xi_{3}^{\prime}\right)^{i_{3}}, \quad \text { for some } \alpha_{i_{1} i_{2} i_{3}} \in \mathrm{CH}^{*}(Y),
$$

where $\xi_{k}^{\prime} \in \mathrm{CH}^{1}\left(E \times_{X} E \times_{X} E\right)$ is the pull-back of $\xi \in \mathrm{CH}^{1}(E)$ from the $k^{\text {th }}$ factor. For any triple $\left(l_{1}, l_{2}, l_{3}\right)$ of non-negative integers, we intersect both sides of $(82)$ with $\left(-E_{1}\right)^{l_{1}}\left(-E_{2}\right)^{l_{2}}\left(-E_{3}\right)^{l_{3}}$ and then apply $\left(\rho^{\times 3}\right)_{*}$ to get

$$
\begin{aligned}
\left(\rho^{\times 3}\right)_{*}\left(\Delta_{123}^{\tilde{X}} \cdot\left(-E_{1}\right)^{l_{1}}\left(-E_{2}\right)^{l_{2}}\left(-E_{3}\right)^{l_{3}}\right)= & \left(\rho^{\times 3}\right)_{*}\left(\left(-E_{1}\right)^{l_{1}}\left(-E_{2}\right)^{l_{2}}\left(-E_{3}\right)^{l_{3}}\right) \cdot \Delta_{123}^{X} \\
& +\left(\rho^{\times 3}\right)_{*}\left(\left(-E_{1}\right)^{l_{1}}\left(-E_{2}\right)^{l_{2}}\left(-E_{3}\right)^{l_{3}} \cdot \iota_{*}^{\prime} \alpha\right) .
\end{aligned}
$$

We note that

$$
\left(\rho^{\times 3}\right)_{*}\left(\Delta_{123}^{\widetilde{X}} \cdot\left(-E_{1}\right)^{l_{1}}\left(-E_{2}\right)^{l_{2}}\left(-E_{3}\right)^{l_{3}}\right)=\left(\iota_{\Delta, 3}\right)_{*} \rho_{*}(-E)^{l_{1}+l_{2}+l_{3}}
$$

and we also observe that $\rho_{*}\left(E^{l}\right) \in \mathrm{CH}_{\mathrm{CK}}^{*}(X)_{0}$ since it is either the class of $X$ (when $l=0$ ) or of the form $i_{*}\left(\right.$ polynomial of $\left.c\left(\mathscr{N}_{Y / X}\right)\right)$.

It follows that

$$
\left(\rho^{\times 3}\right)_{*}\left(\Delta_{123}^{\widetilde{X}} \cdot\left(-E_{1}\right)^{l_{1}}\left(-E_{2}\right)^{l_{2}}\left(-E_{3}\right)^{l_{3}}\right) \in \mathrm{CH}_{\mathrm{CK}}^{*}(X \times X \times X)_{0} .
$$

Similarly, using Proposition 8.7, one also shows that

$$
\left(\rho^{\times 3}\right)_{*}\left(\left(-E_{1}\right)^{l_{1}}\left(-E_{2}\right)^{l_{2}}\left(-E_{3}\right)^{l_{3}}\right) \cdot \Delta_{123}^{X} \in \mathrm{CH}_{\mathrm{CK}}^{*}(X \times X \times X)_{0},
$$

Hence we conclude that

$$
\left(\rho^{\times 3}\right)_{*}\left(\left(-E_{1}\right)^{l_{1}}\left(-E_{2}\right)^{l_{2}}\left(-E_{3}\right)^{l_{3}} \cdot \iota_{*}^{\prime} \alpha\right) \in \mathrm{CH}_{\mathrm{CK}}^{*}(X \times X \times X)_{0} .
$$

Substituting the expression (83) into the above equation, we find

$$
\begin{aligned}
\left(\rho^{\times 3}\right)_{*}\left(\left(-E_{1}\right)^{l_{1}}\left(-E_{2}\right)^{l_{2}}\left(-E_{3}\right)^{l_{3}} \cdot \iota_{*}^{\prime} \alpha\right) & =\left(\rho^{\times 3}\right)_{*} \iota_{*}^{\prime}\left(\left(\xi_{1}^{\prime}\right)^{l_{1}}\left(\xi_{2}^{\prime}\right)^{l_{2}}\left(\xi_{3}^{\prime}\right)^{l_{3}} \cdot \alpha\right) \\
& =\left(\iota_{\Delta, 3}\right)_{*} i_{*}\left(\pi_{/ Y}^{\times 3}\right)_{*}\left(\left(\xi_{1}^{\prime}\right)^{l_{1}}\left(\xi_{2}^{\prime}\right)^{l_{2}}\left(\xi_{3}^{\prime}\right)^{l_{3}} \cdot \alpha\right) \\
& =\left(\iota_{\Delta, 3}\right)_{*} i_{*} L_{l_{1} l_{2} l_{3}}\left(\alpha_{i_{1} i_{2} i_{3}}\right) \in \mathrm{CH}_{\mathrm{CK}}^{*}(X \times X \times X)_{0},
\end{aligned}
$$


where $L_{l_{1} l_{2} l_{3}}$ is a linear form of $\alpha_{i_{1} i_{2} i_{3}}$ whose coefficients are polynomials of the Chern classes of $\mathscr{N}_{Y / X}$. Since both $i_{*}$ and $\left(\iota_{\Delta, 3}\right)_{*}$ are injective and respect the decompositions of the Chow groups, we get

$$
L_{l_{1} l_{2} l_{2}}\left(\alpha_{i_{1} i_{2} i_{3}}\right) \in \mathrm{CH}^{*}(Y)_{0} .
$$

By induction, it follows from these linear equations that $\alpha_{i_{1} i_{2} i_{3}} \in \mathrm{CH}^{*}(Y)_{0}$. Consider the following fiber square

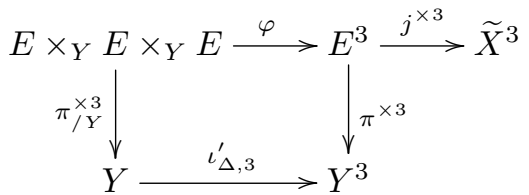

where $\iota_{\Delta, 3}^{\prime}$ is the small diagonal embedding of $Y$. Note that

$$
\begin{aligned}
\iota_{*}^{\prime} \alpha & =\left(j^{\times 3}\right)_{*} \varphi_{*} \alpha \\
& =\left(j^{\times 3}\right)_{*}\left(\sum_{i_{1}, i_{2}, i_{3}=0}^{r} \varphi_{*}\left(\left(\pi_{/ Y}^{\times 3}\right)^{*} \alpha_{i_{1} i_{2} i_{3}}\right) \cdot \xi_{1}^{i_{1}} \xi_{2}^{i_{2}} \xi_{3}^{i_{3}}\right) \\
& =\left(j^{\times 3}\right)_{*}\left(\sum_{i_{1}, i_{2}, i_{3}=0}^{r}\left(\pi^{\times 3}\right)^{*}\left(\left(\iota_{\Delta, 3}^{\prime}\right)_{*} \alpha_{i_{1} i_{2} i_{3}}\right) \cdot \xi_{1}^{i_{1}} \xi_{2}^{i_{2}} \xi_{3}^{i_{3}}\right) .
\end{aligned}
$$

Combining this with (81), (82) and the fact that $\alpha_{i_{1} i_{2} i_{3}} \in \mathrm{CH}^{*}(Y)_{0}$ yields that $\Delta_{123}^{\widetilde{X}}$ lies in the piece $\mathrm{CH}^{2 d}\left(\widetilde{X}^{3}\right)_{0}$, which finishes the proof.

13.3. Multiplicative Chow-Künneth decomposition for $X^{[2]}$. If $X$ is a smooth projective variety, we consider $X^{[2]}$ as the quotient under the action of $\mathfrak{S}_{2}$ that permutes the factors on the blow-up of $X \times X$ along the diagonal. First we note that Proposition 8.7 implies that condition (ii) of Proposition 13.2 is automatically satisfied for the blow-up of $X \times X$ along the diagonal if $X$ is endowed with a multiplicative Chow-Künneth decomposition that is self-dual :

Proposition 13.3. Let $X$ be a smooth projective variety endowed with a multiplicative self-dual ChowKünneth decomposition. Assume that $c_{p}(X)$ belongs to $\mathrm{CH}_{\mathrm{CK}}^{p}(X)_{0}$ for all $p \geq 0$. Then the induced Chow-Künneth decomposition (as described in Proposition 13.2) on the blow-up of $X \times X$ along the diagonal is multiplicative.

Proof. Indeed, by Proposition 8.7, the assumptions of Proposition 13.2 are met (and note also that, since $p_{1} \circ \iota_{\Delta}=\mathrm{id}_{X}: X \rightarrow X,\left(\iota_{\Delta}\right)_{*}: \mathrm{CH}^{*}(X) \rightarrow \mathrm{CH}^{*+d}(X \times X)$ is injective).

Theorem 13.4. Let $X$ be a smooth projective variety endowed with a multiplicative self-dual ChowKünneth decomposition. Assume that $c_{p}(X)$ belongs to $\mathrm{CH}_{\mathrm{CK}}^{p}(X)_{0}$ for all $p \geq 0$. For instance, $X$ could be an abelian variety (the positive Chern classes are trivial) or a K3 surface endowed with the ChowKünneth decomposition $(3)\left(c_{2}(X)=24 \mathfrak{o}_{X}\right.$ by [11]). Then $X^{[2]}$ has a multiplicative Chow-Künneth decomposition.

Proof. Let $\tau: X \times X \rightarrow X \times X$ be the morphism that permutes the factors. This gives an action of the symmetric group $\mathfrak{S}_{2}=\{1, \tau\}$ on $X \times X$, which by functoriality of blow-ups extends to an action of $\mathfrak{S}_{2}$ on the blow-up $\rho: Z=(\widetilde{X \times X})_{\Delta} \rightarrow X \times X$ of $X \times X$ along the diagonal. The morphism $p: Z \rightarrow X^{[2]}$ is then the quotient morphism corresponding to that action.

The action of $\mathfrak{S}_{2}=\{1, \tau\}$ on $X \times X$ induces an action of $\mathfrak{S}_{2}$ on the ring of self-correspondences of $X \times X$ : if we view 1 and $\tau$ as correspondences from $X \times X$ to $X \times X$, then we have

$$
\tau \bullet \gamma:=\tau \circ \gamma \circ \tau, \quad \text { for all } \gamma \in \mathrm{CH}^{2 d}((X \times X) \times(X \times X)) .
$$

It is then clear that the product Chow-Künneth decomposition of $X \times X$ as defined in (48) is $\mathfrak{S}_{2^{-}}$ equivariant for that action ; it is also multiplicative by virtue of Theorem 8.6. Likewise, the induced action of $\mathfrak{S}_{2}$ on the blow-up $Z$ induces an action of $\mathfrak{S}_{2}$ on the ring of self-correspondences of $Z$, and again we view 1 and $\tau$ as correspondences from $Z$ to $Z$. With respect to that action, the Chow-Künneth 
decomposition $\left\{\pi_{Z}^{i}: 0 \leq i \leq 4 d\right\}$ on $Z$ induced by that on $X \times X$ and $X$ via the blow-up formula (79) is $\mathfrak{S}_{2}$-equivariant ; it is also multiplicative by Proposition 13.3. Moreover, as correspondences, we have

$$
\Gamma_{p} \circ{ }^{t} \Gamma_{p}=2 \Delta_{X[2]} \quad \text { and } \quad{ }^{t} \Gamma_{p} \circ \Gamma_{p}=1+\tau,
$$

where $\Gamma_{p} \in \mathrm{CH}^{2 d}\left(Z \times X^{[2]}\right)$ denotes the graph of $p: Z \rightarrow X^{[2]}$ and ${ }^{t} \Gamma_{p}$ denotes its transpose.

We now claim that the correspondences

$$
\pi^{i}:=\frac{1}{2} \Gamma_{p} \circ \pi_{Z}^{i} \circ{ }^{t} \Gamma_{p} \in \mathrm{CH}^{2 d}\left(X^{[2]} \times X^{[2]}\right)
$$

define a multiplicative Chow-Künneth decomposition on $X^{[2]}$. First it is clear that the action of $\pi^{i}$ on $\mathrm{H}^{*}\left(X^{[2]}, \mathbb{Q}\right)$ is the projector on $\mathrm{H}^{i}\left(X^{[2]}, \mathbb{Q}\right)$. Next we have

$$
\begin{aligned}
4 \pi^{i} \circ \pi^{j} & =\Gamma_{p} \circ \pi_{Z}^{i} \circ{ }^{t} \Gamma_{p} \circ \Gamma_{p} \circ \pi_{Z}^{j} \circ{ }^{t} \Gamma_{p}=\Gamma_{p} \circ \pi_{Z}^{i} \circ(1+\tau) \circ \pi_{Z}^{j} \circ{ }^{t} \Gamma_{p} \\
& =\Gamma_{p} \circ \pi_{Z}^{i} \circ \pi_{Z}^{j} \circ{ }^{t} \Gamma_{p}+\Gamma_{p} \circ \pi_{Z}^{i} \circ \tau \circ \pi_{Z}^{j} \circ{ }^{t} \Gamma_{p} .
\end{aligned}
$$

Since $\pi_{Z}^{i}$ is $\mathfrak{S}_{2}$-equivariant, we have $\tau \circ \pi_{Z}^{i} \circ \tau=\pi_{Z}^{i}$, so that $\tau \circ \pi_{Z}^{i}=\pi_{Z}^{i} \circ \tau$. Thus $\Gamma_{p} \circ \pi_{Z}^{i} \circ \tau \circ \pi_{Z}^{j} \circ{ }^{t} \Gamma_{p}=$ $\Gamma_{p} \circ \tau \circ \pi_{Z}^{i} \circ \pi_{Z}^{j} \circ{ }^{t} \Gamma_{p}$, and because $\Gamma_{p} \circ \tau=\Gamma_{p}$, we get $\Gamma_{p} \circ \pi_{Z}^{i} \circ \tau \circ \pi_{Z}^{j} \circ{ }^{t} \Gamma_{p}=\Gamma_{p} \circ \pi_{Z}^{i} \circ \pi_{Z}^{j} \circ{ }^{t} \Gamma_{p}$. This yields

$$
4 \pi^{i} \circ \pi^{j}=2 \Gamma_{p} \circ \pi_{Z}^{i} \circ \pi_{Z}^{j} \circ{ }^{t} \Gamma_{p},
$$

and hence that the correspondences $\pi^{i}$ define a Chow-Künneth decomposition on $X^{[2]}$. It remains to check that this decomposition is multiplicative. By the criterion given in Proposition 8.4, it suffices to check that the small diagonal $\Delta_{123}^{X^{[2]}}$ belongs to $\mathrm{CH}_{\mathrm{CK}}^{4 d}\left(X^{[2]}\right)_{0}$, i.e., that

$$
\left(\pi^{i} \otimes \pi^{j} \otimes \pi^{k}\right)_{*} \Delta_{123}^{X[2]}=0, \quad \text { whenever } i+j+k \neq 4 d .
$$

We note that $\Gamma_{p} \circ \pi_{Z}^{i} \circ \tau=\Gamma_{p} \circ \tau \circ \pi_{Z}^{i}=\Gamma_{p} \circ \pi_{Z}^{i}$ and therefore that

$$
\Gamma_{p} \circ \pi_{Z}^{i} \circ{ }^{t} \Gamma_{p} \circ \Gamma_{p}=\Gamma_{p} \circ \pi_{Z}^{i} \circ(1+\tau)=2 \Gamma_{p} \circ \pi_{Z}^{i} \quad \text { in } \mathrm{CH}^{2 d}\left(Z \times X^{[2]}\right) .
$$

We also note that

$$
\left(\Gamma_{p} \otimes \Gamma_{p} \otimes \Gamma_{p}\right)_{*} \Delta_{123}^{Z}=4 \Delta_{123}^{X^{[2]}}
$$

It follows that

$$
\begin{aligned}
\left(\pi^{i} \otimes \pi^{j} \otimes \pi^{k}\right)_{*} \Delta_{123}^{X} & =\frac{1}{32}\left(\left(\Gamma_{p} \circ \pi_{Z}^{i} \circ{ }^{t} \Gamma_{p}\right) \otimes\left(\Gamma_{p} \circ \pi_{Z}^{j} \circ{ }^{t} \Gamma_{p}\right) \otimes\left(\Gamma_{p} \circ \pi_{Z}^{k} \circ{ }^{t} \Gamma_{p}\right)\right)_{*}(p \times p \times p)_{*} \Delta_{123}^{Z} \\
& =\frac{1}{32}\left(\left(\Gamma_{p} \circ \pi_{Z}^{i} \circ{ }^{t} \Gamma_{p} \circ \Gamma_{p}\right) \otimes\left(\Gamma_{p} \circ \pi_{Z}^{j} \circ{ }^{t} \Gamma_{p} \circ \Gamma_{p}\right) \otimes\left(\Gamma_{p} \circ \pi_{Z}^{k} \circ{ }^{t} \Gamma_{p} \circ \Gamma_{p}\right)\right)_{*} \Delta_{123}^{Z} \\
& =\frac{1}{4}(p \times p \times p)_{*}\left(\pi_{Z}^{i} \otimes \pi_{Z}^{j} \otimes \pi_{Z}^{k}\right)_{*} \Delta_{123}^{Z} \\
& =0 \quad \text { if } i+j+k \neq 4 d,
\end{aligned}
$$

where the last equality follows from multiplicativity for the Chow-Künneth decomposition $\left\{\pi_{Z}^{i}\right\}$ of $Z$. This finishes the proof of the theorem.

Remark 13.5. Define as usual $\mathrm{CH}_{\mathrm{CK}}^{p}\left(X^{[2]}\right)_{s}:=\pi_{*}^{2 p-s} \mathrm{CH}^{p}\left(X^{[2]}\right)$. Then note, for further reference, that with the notations of Theorem 13.4 and its proof the quotient map $p: Z \rightarrow X^{[2]}$ is compatible with the Chow-Künneth decompositions on $Z$ and $X^{[2]}$, in the sense that for all $p$ and all $s$ we have

$$
p_{*} \mathrm{CH}_{\mathrm{CK}}^{p}(Z)_{s}=\mathrm{CH}_{\mathrm{CK}}^{p}\left(X^{[2]}\right)_{s} \quad \text { and } \quad p^{*} \mathrm{CH}_{\mathrm{CK}}^{p}\left(X^{[2]}\right)_{s} \subseteq \mathrm{CH}_{\mathrm{CK}}^{p}(Z)_{s} \text {. }
$$

Remark 13.6. Let $C$ be a smooth projective curve. The Hilbert scheme of length- $n$ subschemes on $C$ is smooth and is nothing but the $n^{\text {th }}$ symmetric product of $C$. Assume that $C$ admits a multiplicative ChowKünneth decomposition, e.g., $C$ is a hyperelliptic curve ; see Example 8.16. Then the product ChowKünneth decomposition on $C^{n}$ is multiplicative by Theorem 8.6, and it is clearly $\mathfrak{S}_{n}$-equivariant. Here, $\mathfrak{S}_{n}$ is the symmetric group on $n$ elements acting on $C^{n}$ by permuting the factors. An easy adaptation of the proof of Theorem 13.4 yields that $C^{[n]}$ has a multiplicative Chow-Künneth decomposition. 
13.4. Proof of Theorem 6. According to Theorem 8.6, Proposition 13.1, Proposition 13.3 and Theorem 13.4 , it suffices to check that

(a) the varieties listed in Theorem 6 admit a multiplicative Chow-Künneth decomposition such that their Chern classes belong to the degree-zero graded part of the induced decomposition on the Chow ring ;

(b) the property that the Chern classes belong to the degree-zero graded part of the Chow ring is stable under the operations (i)-(iv) listed in Theorem 6.

Item (b) is taken care of by the following lemma :

Lemma 13.7. Let $X$ and $Y$ be two smooth projective varieties endowed with multiplicative ChowKünneth decompositions such that $c_{i}(X) \in \mathrm{CH}_{\mathrm{CK}}^{i}(X)_{0}$ for all $0 \leq i \leq \operatorname{dim} X$ and $c_{i}(Y) \in \mathrm{CH}_{\mathrm{CK}}^{i}(Y)_{0}$ for all $0 \leq i \leq \operatorname{dim} Y$. Then the following statements hold.

(i) $c_{i}(X \times Y) \in \mathrm{CH}_{\mathrm{CK}}^{i}(X \times Y)_{0}$ for all $0 \leq i \leq \operatorname{dim} X+\operatorname{dim} Y$.

(ii) $c_{i}\left(\mathbb{P}\left(\mathscr{T}_{X}\right)\right) \in \mathrm{CH}_{\mathrm{CK}}^{i}\left(\mathbb{P}\left(\mathscr{T}_{X}\right)\right)_{0}$ for all $0 \leq i \leq 2 \operatorname{dim} X-1$.

(iii) $c_{i}(Z) \in \mathrm{CH}_{\mathrm{CK}}^{i}(Z)_{0}$ for all $0 \leq i \leq 2 \operatorname{dim} X$, where $Z$ is the blow-up of $X \times X$ along the diagonal.

(iv) $c_{i}\left(X^{[2]}\right) \in \mathrm{CH}_{\mathrm{CK}}^{i}\left(X^{[2]}\right)_{0}$ for all $0 \leq i \leq 2 \operatorname{dim} X$.

Proof. Statement (i) follows directly from Theorem 8.6 and Proposition 8.7(i), and from the formula $c(X \times Y)=p_{1}^{*} c(X) \cdot p_{2}^{*} c(Y)$, where $p_{1}: X \times Y \rightarrow X$ and $p_{2}: X \times Y \rightarrow Y$ are the two projections.

Let $E:=\mathbb{P}\left(\mathscr{T}_{X}\right)$ be the geometric projectivization of the tangent bundle of $X$ and let $\pi: E \rightarrow X$ be the projection morphism. The relative Euler exact sequence in this setting becomes

$$
0 \longrightarrow \mathcal{O}_{E} \longrightarrow \pi^{*} \mathscr{T}_{X} \otimes \mathcal{O}(1) \longrightarrow \mathscr{T}_{E / X} \longrightarrow 0
$$

where $\mathscr{T}_{E / X}$ is the relative (or vertical) tangent bundle. It follows that

$$
\operatorname{ch}\left(\mathscr{T}_{E / X}\right)=\pi^{*} \operatorname{ch}\left(\mathscr{T}_{X}\right) \cdot e^{\xi}-1,
$$

where $\xi$ is the first Chern class of the relative $\mathcal{O}(1)$-bundle. Note that the multiplicative Chow-Künneth decomposition on $E$ given by Proposition 13.1 satisfies $\xi \in \mathrm{CH}_{\mathrm{CK}}^{1}(E)_{0}$. Hence we conclude that the Chern character $\operatorname{ch}\left(\mathscr{T}_{E / X}\right)$ lies in the piece $\mathrm{CH}_{\mathrm{CK}}^{*}(E)_{0}$, so that

$$
\operatorname{ch}\left(\mathscr{T}_{E}\right)=\pi^{*} \operatorname{ch}\left(\mathscr{T}_{X}\right)+\operatorname{ch}\left(\mathscr{T}_{E / X}\right) \in \mathrm{CH}_{\mathrm{CK}}^{*}(E)_{0} .
$$

Statement (ii) follows immediately.

Let $\rho: Z \rightarrow X \times X$ be the blow-up morphism and let $j^{\prime}: E \rightarrow Z$ be the exceptional divisor. Note that $E$ is isomorphic to $\mathbb{P}\left(\mathscr{T}_{X}\right)$. Consider the short exact sequence

$$
0 \longrightarrow \rho^{*} \Omega_{X \times X}^{1} \longrightarrow \Omega_{Z}^{1} \longrightarrow j_{*}^{\prime} \Omega_{E / X}^{1} \longrightarrow 0 .
$$

By taking Chern characters, one finds

$$
\operatorname{ch}\left(\Omega_{Z}^{1}\right)=\rho^{*} \operatorname{ch}\left(\Omega_{X \times X}^{1}\right)+\operatorname{ch}\left(j_{*}^{\prime} \Omega_{E / X}^{1}\right) .
$$

By (i) and Proposition 13.3, we see that

$$
\rho^{*} \operatorname{ch}\left(\Omega_{X \times X}^{1}\right) \in \mathrm{CH}_{\mathrm{CK}}^{*}(Z)_{0} .
$$

Hence it suffices to show that

$$
\operatorname{ch}\left(j_{*}^{\prime} \Omega_{E / X}^{1}\right) \in \mathrm{CH}_{\mathrm{CK}}^{*}(Z)_{0} .
$$

This can be obtained from the Grothendieck-Riemann-Roch Theorem. Indeed, we have

$$
\operatorname{ch}\left(j_{*}^{\prime} \Omega_{E / X}^{1}\right)=\frac{j_{*}^{\prime}\left(\operatorname{ch}\left(\Omega_{E / X}^{1}\right) \cdot \operatorname{td}(E)\right)}{\operatorname{td}(Z)}=j_{*}^{\prime}\left(\frac{\operatorname{ch}\left(\Omega_{E / X}^{1}\right)}{\operatorname{td}\left(\mathscr{N}_{E / Z}\right)}\right) .
$$

Now note that $\mathscr{N}_{E / Z}=\mathcal{O}_{E}(-1)$, so that $\operatorname{td}\left(\mathscr{N}_{E / Z}\right) \in \mathrm{CH}_{\mathrm{CK}}^{*}(E)_{0}$. Statement (ii) implies that $\operatorname{ch}\left(\Omega_{E / X}^{1}\right) \in$ $\mathrm{CH}_{\mathrm{CK}}^{*}(E)_{0}$. The proof of (iii) then follows from the compatibility of $j_{*}^{\prime}$ with the gradings induced by the Chow-Künneth decompositions as in Proposition 13.2. 
Let $p: Z \rightarrow X^{[2]}$ be the natural double cover ramified along the divisor $E$. Then we have the following short exact sequence

$$
0 \longrightarrow \mathscr{T}_{Z} \longrightarrow p^{*} \mathscr{T}_{X[2]} \longrightarrow \mathcal{O}_{E}(2 E) \longrightarrow 0,
$$

from which one obtains

$$
p^{*} \operatorname{ch}\left(\mathscr{T}_{X[2]}\right)=\operatorname{ch}\left(\mathscr{T}_{Z}\right)+\operatorname{ch}\left(\mathcal{O}_{E}(2 E)\right) .
$$

Note that $\mathcal{O}_{E}(2 E)$ fits into the short exact sequences

$$
0 \longrightarrow \mathcal{O}_{Z}(E) \longrightarrow \mathcal{O}_{Z}(2 E) \longrightarrow \mathcal{O}_{E}(2 E) \longrightarrow 0
$$

It follows that $\operatorname{ch}\left(\mathcal{O}_{E}(2 E)\right) \in \mathrm{CH}_{\mathrm{CK}}^{*}(Z)_{0}$. Statement (iii) implies that $\operatorname{ch}\left(\mathscr{T}_{Z}\right) \in \mathrm{CH}_{\mathrm{CK}}^{*}(Z)_{0}$. Hence we get

$$
p^{*} \operatorname{ch}\left(\mathscr{T}_{X[2]}\right) \in \mathrm{CH}_{\mathrm{CK}}^{*}(Z)_{0},
$$

which proves (iv) ; see Remark 13.5.

As for item (a), first note that if $X$ has a Chow-Künneth decomposition, we have $\mathrm{CH}^{0}(X)=\mathrm{CH}_{\mathrm{CK}}^{0}(X)_{0}$ so that obviously $c_{0}(X)$ lies in $\mathrm{CH}_{\mathrm{CK}}^{0}(X)_{0}$. We then proceed case-by-case :

Case 1: $X$ is a smooth projective variety with Chow groups of finite rank (as Q-vector spaces). By [49, Theorem 5], the Chow motive of $X$ is isomorphic to a direct sum of Lefschetz motives (see [49] for the definitions) so that $X$ has a Chow-Künneth decomposition such that the induced grading on $\mathrm{CH}^{*}(X)$ is concentrated in degree zero, that is, $\mathrm{CH}^{*}(X)=\mathrm{CH}_{\mathrm{CK}}^{*}(X)_{0}$. The Chow motive of $X \times X \times X$, which is the 3 -fold self tensor product of the Chow motive of $X$, is also isomorphic to a direct sum of Lefschetz motives and the product Chow-Künneth decomposition is such that $\mathrm{CH}^{*}(X \times X \times X)$ is concentrated in degree 0. It is then clear that the Chern classes of $X$ lie in the degree-zero graded part and in view of the criterion of Proposition 8.4 that this Chow-Künneth decomposition is multiplicative.

Case 2: $X$ is the $n^{\text {th }}$-symmetric product of an hyperelliptic curve $C$. By Remark 13.6, $X=C^{[n]}$ admits a multiplicative Chow-Künneth decomposition. The Chern classes $c_{i}\left(C^{[n]}\right)$ belong to $\mathrm{CH}_{\mathrm{CK}}^{i}\left(C^{[n]}\right)_{0}$ for the following reason. Let $Z \subset C^{[n]} \times C$ be the universal family. Given a line bundle $\mathscr{L}$ on $C$, the associated tautological bundle $\mathscr{L}^{[n]}$ on $C^{[n]}$ can be defined by

$$
\mathscr{L}^{[n]}:=p_{*}\left(\mathcal{O}_{Z} \otimes q^{*} \mathscr{L}\right),
$$

where $p: C^{[n]} \times C \rightarrow C^{[n]}$ and $q: C^{[n]} \times C \rightarrow C$ are the two projections. If we take $\mathscr{L}=\Omega_{C}^{1}$, it turns out that the associated tautological bundle is $\Omega_{C[n]}^{1}$. The Grothendieck-Riemann-Roch theorem applied to this situation becomes

$$
\operatorname{ch}\left(\Omega_{C[n]}^{1}\right)=p_{*}\left(\operatorname{ch}\left(\mathcal{O}_{Z}\right) \cdot q^{*}\left(\operatorname{ch}\left(\Omega_{C}^{1}\right) \cdot \operatorname{td}(C)\right)\right) .
$$

Hence it suffices to see that $\operatorname{ch}\left(\mathcal{O}_{Z}\right) \in \mathrm{CH}_{\mathrm{CK}}^{*}\left(C^{[n]} \times C\right)_{0}$. Let $Z_{i}:=p_{i, n+1}^{-1} \Delta_{C} \subset C^{n} \times C, 1 \leq i \leq n$, and let $\pi: C^{n} \rightarrow C^{[n]}$ be the symmetrization morphism. Then we have

$$
\left(\pi \times \operatorname{Id}_{C}\right)^{*} \mathcal{O}_{Z}=\sum_{i=1}^{n} \mathcal{O}_{Z_{i}}, \quad \text { in } \mathrm{K}_{0}\left(C^{n} \times C\right) .
$$

By taking the Chern character, we see that $\left(\pi \times \operatorname{Id}_{C}\right)^{*} \operatorname{ch}\left(\mathcal{O}_{Z}\right)$ lies in the degree-zero graded part of the Chow ring of $C^{n} \times C$ and hence, after applying $\left(\pi \times \operatorname{Id}_{C}\right)_{*}$, that $\operatorname{ch}\left(\mathcal{O}_{Z}\right) \in \mathrm{CH}_{\mathrm{CK}}^{*}\left(C^{[n]} \times C\right)_{0}$.

Case 3: $X$ is an abelian variety endowed with the multiplicative self-dual Chow-Künneth decomposition of Example 8.3. In that case, the tangent bundle of $X$ is trivial so that its Chern classes of positive degree vanish and hence obviously lie in the degree-zero graded part of $\mathrm{CH}^{*}(X)$.

Case 4: $X$ is a K3 surface endowed with the multiplicative self-dual Chow-Künneth decomposition of Example 8.17. In that case, $c_{1}(X)=0$, and $c_{2}(X)=24 \mathfrak{o}_{X}$ by Beauville-Voisin [11].

The proof of Theorem 6 is now complete. 


\section{The Fourier Decomposition For $S^{[2]}$}

In this section, we construct a cycle $L \in \mathrm{CH}^{2}\left(S^{[2]} \times S^{[2]}\right)$ that represents the Beauville-Bogomolov form $\mathfrak{B}$ and show that $S^{[2]}$ endowed with that cycle $L$ satisfies the conclusions of Theorem 1 and Theorem 2. This is achieved in $§ 14.2$. A preliminary crucial ingredient for that purpose is an equality, given in Lemma 14.3, in the ring of correspondences of $S^{[2]}$.

14.1. A key lemma. Let $X$ be a smooth projective variety of dimension $d$. The set-up is that of Section 10. We consider the diagonals (introduced in of Definition 8.8) $\Delta_{123}, \Delta_{i j}$ and $\Delta_{i}$ with respect to the choice of a zero-cycle $\mathfrak{o}_{X}$ of degree 1 on $X$. For simplicity of notations, from now on we assume that $\mathfrak{o}_{X}$ is effective. Otherwise, one may replace $X_{\mathfrak{o}}$ by $p_{*} q^{*} \mathfrak{o}_{X}$ and Lemma 14.2 below still holds true.

The following blow-up formula, which was already used in the proof of Proposition 13.2, will be very useful in explicit computations. We include it here for the convenience of the reader.

Lemma 14.1 ([25], Proposition 6.7). Let $Y$ be a smooth projective variety, and $Y_{1} \subset Y$ a smooth closed sub-variety of codimension e. Let $\rho_{1}: \widetilde{Y} \rightarrow Y$ be the blow-up of $Y$ along $Y_{1}$ and $E_{1}=\mathbb{P}\left(\mathscr{N}_{Y_{1} / Y}\right) \subset \widetilde{Y}$ the exceptional divisor. We have the following diagram attached to this situation

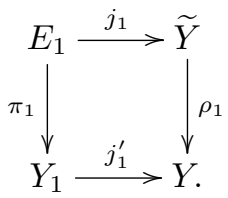

Then for any $\sigma \in \mathrm{CH}^{*}\left(Y_{1}\right)$ we have

$$
\rho_{1}^{*} j_{1, *}^{\prime} \sigma=j_{1, *}\left(c_{e-1}(\mathscr{F}) \cdot \pi_{1}^{*} \sigma\right)
$$

where $\mathscr{F}=\left(\pi_{1}^{*} \mathscr{N}_{Y_{1} / Y}\right) / \mathscr{N}_{E_{1} / \widetilde{Y}}$.

We fix some notations that will be used frequently in the remaining part of this section. Let $f: Y \rightarrow Y^{\prime}$ be a morphism, then $\mathscr{N}_{f}$ denotes the cokernel of the sheaf homomorphism $\mathscr{T}_{Y} \rightarrow f^{*} \mathscr{T}_{Y^{\prime}}$. This quotient sheaf $\mathscr{N}_{f}$ will be called the normal sheaf (or normal bundle if it is locally free) of the morphism $f$. If two varieties $Y_{1}$ and $Y_{2}$ are defined over some base $B$, then $p_{Y_{1} \times_{B} Y_{2}, i}, i=1$, 2, denote the projections $Y_{1} \times{ }_{B} Y_{2} \rightarrow Y_{i}$

Consider the following diagram ( $c f . \S 10$ for the notations) :

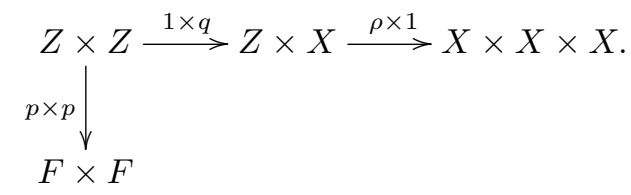

Lemma 14.2. The following equations hold in $\mathrm{CH}^{2 d}(F \times F)$.

$$
\begin{aligned}
(p \times p)_{*}(\rho \times q)^{*} \Delta_{123} & =\sum_{i=0}^{d-1} \frac{(-1)^{d-1-i}}{2} p_{1}^{*}\left(j_{*} \pi^{*} c_{i}(X)\right) \cdot \delta_{1}^{d-1-i} \cdot I \\
(p \times p)_{*}(\rho \times q)^{*} \Delta_{12} & =\sum_{i=0}^{d-1}(-1)^{d-1-i} p_{1}^{*}\left(j_{*} \pi^{*} c_{i}(X)\right) \cdot \delta_{1}^{d-1-i} \cdot p_{2}^{*} X_{\mathfrak{o}} \\
(p \times p)_{*}(\rho \times q)^{*} \Delta_{23} & =p_{1}^{*} X_{\mathfrak{o}} \cdot I-\Gamma_{\mathfrak{o}_{X}} \\
(p \times p)_{*}(\rho \times q)^{*} \Delta_{13} & =p_{2}^{*} X_{\mathfrak{o}} \cdot I-\Gamma_{\mathfrak{o}_{X}} \\
(p \times p)_{*}(\rho \times q)^{*} \Delta_{i} & =p_{1}^{*} X_{\mathfrak{o}} \cdot p_{2}^{*} X_{\mathfrak{o}}, \quad i=1,2 \\
(p \times p)_{*}(\rho \times q)^{*} \Delta_{3} & =2 p_{1}^{*} \mathfrak{o}_{F} .
\end{aligned}
$$


Proof. Let $\iota_{\Delta_{X}} \times \operatorname{Id}_{X}: X \times X \rightarrow X \times X \times X$ be the morphism $(x, y) \mapsto(x, x, y)$. Then we have the following commutative diagram

$$
\begin{aligned}
& E \times Z \stackrel{\operatorname{Id}_{E} \times q}{\longrightarrow} E \times X \stackrel{\pi \times \operatorname{Id}_{X}}{\longrightarrow} X \times X \\
& j^{\prime} \times \operatorname{Id}_{Z} \downarrow \quad j^{\prime} \times \operatorname{Id}_{X} \downarrow \quad \iota_{\Delta_{X}} \times \operatorname{Id}_{X} \downarrow \\
& Z \times Z \stackrel{\operatorname{Id}_{Z} \times q}{\longrightarrow} Z \times X \stackrel{\rho \times \operatorname{Id}_{X}}{\longrightarrow} X \times X \times X,
\end{aligned}
$$

where all the squares are fiber products. Note that $\left(\iota_{\Delta_{X}} \times \operatorname{Id}_{X}\right)_{*} \Delta_{X}=\Delta_{123}$.

$$
\left(\rho \times \operatorname{Id}_{X}\right)^{*} \Delta_{123}=\left(\rho \times \operatorname{Id}_{X}\right)^{*}\left(\iota_{\Delta_{X}} \times \operatorname{Id}_{X}\right)_{*} \Delta_{X} .
$$

We also see that the morphism $\rho \times \operatorname{Id}_{X}$ is naturally the blow-up of $X \times X \times X$ along the image of $\iota_{\Delta_{X}} \times \operatorname{Id}_{X}$. By the blow-up formula (Lemma 14.1), we have

$$
\left(\rho \times \operatorname{Id}_{X}\right)^{*}\left(\iota_{\Delta_{X}} \times \operatorname{Id}_{X}\right)_{*} \Delta_{X}=\left(j^{\prime} \times \operatorname{Id}_{X}\right)_{*}\left(c_{d-1}(\mathscr{E}) \cdot\left(\pi \times \operatorname{Id}_{X}\right)^{*} \Delta_{X}\right),
$$

where $\mathscr{E}$ is the locally free sheaf defined by

$$
\mathscr{E}:=\operatorname{coker}\left\{\mathscr{N}_{j^{\prime} \times \operatorname{Id}_{X}} \longrightarrow\left(\pi \times \operatorname{Id}_{X}\right)^{*} \mathscr{N}_{\iota_{\Delta_{X}} \times \operatorname{Id}_{X}}\right\} .
$$

Note that $\mathscr{N}_{j^{\prime} \times \operatorname{Id}_{X}}=\left(p_{E \times X, 1}\right)^{*} \mathscr{N}_{E / Z}$ and $\mathscr{N}_{\iota_{X} \times \operatorname{Id}_{X}}=\left(p_{X \times X, 1}\right)^{*} \mathscr{T}_{X}$. On $E=\mathbb{P}\left(\mathscr{T}_{X}\right)$ we have the following short exact sequence

$$
0 \longrightarrow \mathcal{O}(-1) \longrightarrow \pi^{*} \mathscr{T}_{X} \longrightarrow \mathscr{E}_{0} \longrightarrow 0
$$

Then the vector bundle $\mathscr{E}$ can be identified with $\left(p_{E \times X, 1}\right)^{*} \mathscr{E}_{0}$. The above short exact sequence implies

$$
c\left(\mathscr{E}_{0}\right)=\pi^{*}\left(1+c_{1}(X)+c_{2}(X)+\cdots+c_{d}(X)\right) \cdot\left(1+\xi+\xi^{2}+\cdots+\xi^{2 d-1}\right),
$$

where $\xi=-\left.E\right|_{E}$ is the first Chern class of the relative $\mathcal{O}(1)$-bundle. It follows that

$$
c_{d-1}(\mathscr{E})=\sum_{i=0}^{d-1}\left(p_{E \times X, 1}\right)^{*}\left(\pi^{*} c_{i}(X) \cdot \xi^{d-1-i}\right) .
$$

Substituting (88) into (86) gives

$$
\begin{aligned}
\left(\rho \times \operatorname{Id}_{X}\right)^{*}\left(\iota_{\Delta_{X}} \times \operatorname{Id}_{X}\right)_{*} \Delta_{X} & =\sum_{i=0}^{d-1}\left(j^{\prime} \times \operatorname{Id}_{X}\right)_{*}\left\{\left(p_{E \times X, 1}\right)^{*}\left(\pi^{*} c_{i}(X) \cdot \xi^{d-1-i}\right) \cdot\left(\pi \times \operatorname{Id}_{X}\right)^{*} \Delta_{X}\right\} \\
& =\sum_{i=0}^{d-1}\left(j^{\prime} \times \operatorname{Id}_{X}\right)_{*}\left\{\left(p_{E \times X, 1}\right)^{*}\left(\pi^{*} c_{i}(X) \cdot \xi^{d-1-i}\right) \cdot\left(\operatorname{Id}_{E}, \pi\right)_{*} E\right\} \\
& =\sum_{i=0}^{d-1}\left(j^{\prime} \times \operatorname{Id}_{X}\right)_{*}\left(\operatorname{Id}_{E}, \pi\right)_{*}\left\{\left(\operatorname{Id}_{E}, \pi\right)^{*}\left(p_{E \times X, 1}\right)^{*}\left(\pi^{*} c_{i}(X) \cdot \xi^{d-1-i}\right)\right\} \\
& =\sum_{i=0}^{d-1}\left(j^{\prime}, \pi\right)_{*}\left(\pi^{*} c_{i}(X) \cdot \xi^{d-1-i}\right) .
\end{aligned}
$$

Here the second equality uses the following fiber product square

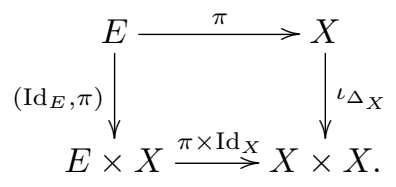

The third equality uses the projection formula. The last equality follows from the facts that $\left(j^{\prime} \times \operatorname{Id}_{X}\right) \circ$ $\left(\operatorname{Id}_{E}, \pi\right)=\left(j^{\prime}, \pi\right)$ and that $\left(p_{E \times X, 1}\right) \circ\left(\operatorname{Id}_{E}, \pi\right)=\operatorname{Id}_{E}$. Substituting the above equality into equation (85) yields

$$
\left(\rho \times \operatorname{Id}_{X}\right)^{*} \Delta_{123}=\sum_{i=0}^{d-1}\left(j^{\prime}, \pi\right)_{*}\left(\pi^{*} c_{i}(X) \cdot \xi^{d-1-i}\right) .
$$


Note that we have the following fiber square

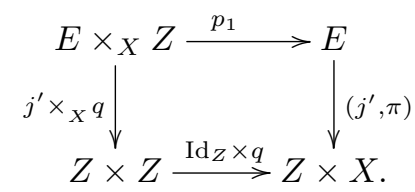

From this and the above equation (89), we get

$$
\left(\operatorname{Id}_{Z} \times q\right)^{*}\left(\rho \times \operatorname{Id}_{X}\right)^{*} \Delta_{123}=\sum_{i=0}^{d-1}\left(j^{\prime} \times_{X} q\right)_{*}\left(p_{1}\right)^{*}\left(\pi^{*} c_{i}(X) \cdot \xi^{d-1-i}\right) .
$$

We now observe that, for any cycle class $\mathfrak{a}$ on $E$, we have

$$
\left(j^{\prime} \times_{X} q\right)_{*}\left(p_{1}\right)^{*} \mathfrak{a}=\left(p_{Z \times Z, 1}\right)^{*}\left(j_{*}^{\prime} \mathfrak{a}\right) \cdot(q \times q)^{*} \Delta_{X}
$$

Indeed, consider the following commutative diagram

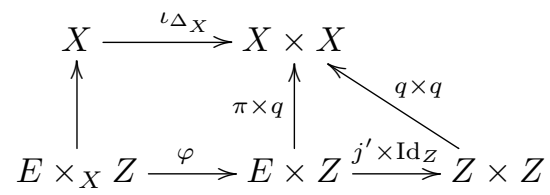

where the square is a fiber product. Then we have

$$
\begin{aligned}
\left(j^{\prime} \times{ }_{X} q\right)_{*} p_{1}^{*} \mathfrak{a} & =\left(j^{\prime} \times \operatorname{Id}_{Z}\right)_{*} \varphi_{*} p_{1}^{*} \mathfrak{a} \\
& =\left(j^{\prime} \times \operatorname{Id}_{Z}\right)_{*}\left(\left(p_{E \times Z, 1}\right)^{*} \mathfrak{a} \cdot(\pi \times q)^{*} \Delta_{X}\right) \\
& =\left(j^{\prime} \times \operatorname{Id}_{Z}\right)_{*}\left(\left(p_{E \times Z, 1}\right)^{*} \mathfrak{a} \cdot\left(j^{\prime} \times \operatorname{Id}_{Z}\right)^{*}(q \times q)^{*} \Delta_{X}\right) \\
& =\left(j^{\prime} \times \operatorname{Id}_{Z}\right)_{*}\left(p_{E \times Z, 1}\right)^{*} \mathfrak{a} \cdot(q \times q)^{*} \Delta_{X} .
\end{aligned}
$$

Then equation (91) follows easily by noting

$$
\left(j^{\prime} \times \operatorname{Id}_{Z}\right)_{*}\left(p_{E \times Z, 1}\right)^{*} \mathfrak{a}=\left(p_{Z \times Z, 1}\right)^{*} j_{*}^{\prime} \mathfrak{a} .
$$

Combining equations (90) and (91) gives

$$
\begin{aligned}
(\rho \times q)^{*} \Delta_{123} & =\sum_{i=0}^{d-1}\left(p_{Z \times Z, 1}\right)^{*}\left(j_{*}^{\prime}\left(\pi^{*} c_{i}(X) \cdot \xi^{d-1-i}\right)\right) \cdot(q \times q)^{*} \Delta_{X} \\
& =\sum_{i=0}^{d-1}\left(p_{Z \times Z, 1}\right)^{*}\left(j_{*}^{\prime}\left(\pi^{*} c_{i}(X) \cdot j^{\prime *}(-E)^{d-1-i}\right)\right) \cdot(q \times q)^{*} \Delta_{X} \\
& =\sum_{i=0}^{d-1}(-1)^{d-1-i}\left(p_{Z \times Z, 1}\right)^{*}\left(j_{*}^{\prime} \pi^{*} c_{i}(X) \cdot E^{d-1-i}\right) \cdot(q \times q)^{*} \Delta_{X} \\
& =\sum_{i=0}^{d-1} \frac{(-1)^{d-1-i}}{2}(p \times p)^{*}\left(p_{1}^{*} j_{*} \pi^{*} c_{i}(X) \cdot \delta_{1}^{d-1-i}\right) \cdot(q \times q)^{*} \Delta_{X} .
\end{aligned}
$$

Now we apply $(p \times p)_{*}$ to the above equation and find

$$
(p \times p)_{*}(\rho \times q)^{*} \Delta_{123}=\sum_{i=0}^{d-1} \frac{(-1)^{d-1-i}}{2} p_{1}^{*}\left(j_{*} \pi^{*} c_{i}(X)\right) \cdot \delta_{1}^{d-1-i} \cdot(p \times p)_{*}(q \times q)^{*} \Delta_{X} .
$$

This proves the first identity. 
In order to prove the second equality, we note that $\Delta_{12}=\left(\iota_{\Delta_{X}} \times \operatorname{Id}_{X}\right)_{*}\left(p_{X \times X, 2}\right)^{*} \mathfrak{o}_{X}$. Hence we have

$$
\begin{aligned}
\left(\rho \times \operatorname{Id}_{X}\right)^{*} \Delta_{12} & =\left(\rho \times \operatorname{Id}_{X}\right)^{*}\left(\iota_{\Delta_{X}} \times \operatorname{Id}_{X}\right)_{*}\left(p_{X \times X, 2}\right)^{*} \mathfrak{o}_{X} \\
& =\left(j^{\prime} \times \operatorname{Id}_{X}\right)_{*}\left(c_{d-1}(\mathscr{E}) \cdot\left(\pi \times I d_{X}\right)^{*}\left(p_{X \times X, 2}\right)^{*} \mathfrak{o}_{X}\right) \\
& =\sum_{i=0}^{d-1}(-1)^{d-1-i}\left(j^{\prime} \times \operatorname{Id}_{X}\right)_{*}\left\{\left(p_{E \times X, 1}\right)^{*}\left(\pi^{*} c_{i}(X) \cdot E^{d-1-i}\right) \cdot\left(p_{E \times X, 2}\right)^{*} \mathfrak{o}_{X}\right\} \\
& =\sum_{i=0}^{d-1}(-1)^{d-1-i}\left(p_{Z \times X, 1}\right)^{*}\left(j_{*}^{\prime} \pi^{*} c_{i}(X) \cdot E^{d-1-i}\right) \cdot\left(p_{Z \times X, 2}\right)^{*} \mathfrak{o}_{X} .
\end{aligned}
$$

Applying $\left(\operatorname{Id}_{Z} \times q\right)^{*}$ to the above equation, we get

$$
(\rho \times q)^{*} \Delta_{12}=\sum_{i=0}^{d-1}(-1)^{d-1-i}\left(p_{Z \times Z, 1}\right)^{*}\left(j_{*}^{\prime} \pi^{*} c_{i}(X) \cdot E^{d-1-i}\right) \cdot\left(p_{Z \times Z, 2}\right)^{*}\left(q^{*} \mathfrak{o}_{X}\right) .
$$

It follows from this equation that

$$
(p \times p)_{*}(\rho \times q)^{*} \Delta_{12}=\sum_{i=0}^{d-1}(-1)^{d-1-i} p_{1}^{*}\left(j_{*} \pi^{*} c_{i}(X)\right) \cdot \delta_{1}^{d-1-i} \cdot p_{2}^{*}\left(p_{*} q^{*} \mathfrak{o}_{X}\right),
$$

which proves the second equality.

For the third equality, we proceed to the following direct computation. Recall that there is an involution $\tau$ on $Z$. We will denote $\tau_{1}:=\tau \times \operatorname{Id}_{Z}$ the involution of $Z \times Z$ induced by the action of $\tau$ on the first factor. Similarly, we have $\tau_{2}:=\operatorname{Id}_{Z} \times \tau$. Then we have

$$
\begin{aligned}
(p \times p)_{*}(\rho \times q)^{*} \Delta_{23}= & (p \times p)_{*}(\rho \times q)^{*}\left\{\left(p_{X^{3}, 1}\right)^{*} \mathfrak{o}_{X} \cdot\left(p_{X^{3}, 23}\right)^{*} \Delta_{X}\right\} \\
= & (p \times p)_{*}\left\{\left(p_{Z \times Z, 1}\right)^{*} q^{*} \mathfrak{o}_{X} \cdot \tau_{1}^{*}(q \times q)^{*} \Delta_{X}\right\} \\
= & (p \times p)_{*}\left\{(p \times p)^{*} p_{1}^{*} p_{*} q^{*} \mathfrak{o}_{X} \cdot \tau_{1}^{*}(q \times q)^{*} \Delta_{X}\right\} \\
& \quad-(p \times p)_{*}\left\{\tau_{1}^{*}\left(p_{Z \times Z, 1}\right)^{*} q^{*} \mathfrak{o}_{X} \cdot \tau_{1}^{*}(q \times q)^{*} \Delta_{X}\right\} \\
= & p_{1}^{*} p_{*} q^{*} \mathfrak{o}_{X} \cdot(p \times p)_{*} \tau_{1}^{*}(q \times q)^{*} \Delta_{X} \\
& \quad-(p \times p)_{*}\left\{\left(p_{Z \times Z, 1}\right)^{*} q^{*} \mathfrak{o}_{X} \cdot(q \times q)^{*} \Delta_{X}\right\} \\
= & p_{1}^{*}\left(p_{*} q^{*} \mathfrak{o}_{X}\right) \cdot I-\Gamma_{\mathfrak{o}_{X}} .
\end{aligned}
$$

Here the first equality follows from the definition of $\Delta_{23}$. The second equality follows from the fact that $\left(p_{X^{3}, 1}\right) \circ(\rho \times q)=q \circ\left(p_{Z \times Z, 1}\right)$ and that $\left(p_{X^{3}, 1}\right) \circ(\rho \times q)=(q \times q) \circ \tau_{1}$. The third equality is obtained by using

$$
(p \times p)^{*} p_{1}^{*} p_{*} \mathfrak{a}=\left(p_{Z \times Z, 1}\right)^{*} \mathfrak{a}+\tau_{1}^{*}\left(p_{Z \times Z, 1}\right)^{*} \mathfrak{a}, \quad \forall \mathfrak{a} \in \mathrm{CH}^{*}(Z \times Z) .
$$

The fourth equality uses the projection formula and the fact that $(p \times p)_{*} \tau_{1}^{*} \mathfrak{a}=(p \times p)_{*} \mathfrak{a}$ for all cycle classes $\mathfrak{a}$ on $Z \times Z$. The last equality follows from the definition of $\Gamma_{\sigma}$.

The equation involving $\Delta_{13}$ follows from that involving $\Delta_{23}$ by symmetry. The remaining equalities are proved easily.

Note that a direct computation shows that, if $X$ satisfies Assumption 12.3, then for all $\sigma \in \mathrm{CH}_{0}(F)$ we have

$$
\left(p_{1}^{*}\left(2 X_{\mathfrak{o}}+(-1)^{d} \delta^{d}\right) \cdot I\right)^{*} \sigma=\left(2 X_{\mathfrak{o}}+(-1)^{d} \delta^{d}\right) \cdot I_{*} \sigma=2 \operatorname{deg}(\sigma) \mathfrak{o}_{F} .
$$

The reason for carrying out the computations involved in the proof of Lemma 14.2 is to show that the formula above can actually be made much more precise in the case when $X=S$ is a K3 surface. The following lemma gives an equality of correspondences (rather than merely an equality of actions of correspondences on zero-cycles) and is essential to establishing Conjecture 1 for $F=S^{[2]}$.

Lemma 14.3. Let $S$ be a K3 surface and $F=S^{[2]}$ the Hilbert scheme of length-2 subschemes of $S$. Then the following relation holds in $\mathrm{CH}^{4}(F \times F)$ :

$$
p_{1}^{*}\left(2 S_{\mathfrak{o}}+\delta^{2}\right) \cdot I=2 \delta_{1}^{2} \cdot p_{2}^{*} S_{\mathfrak{o}}+4 p_{1}^{*} S_{\mathfrak{o}} \cdot p_{2}^{*} S_{\mathfrak{o}}+2 p_{1}^{*} \mathfrak{o}_{F} .
$$


Proof. We know from [11] that $\Delta_{\text {tot }}=0 \in \mathrm{CH}_{2}(S \times S \times S)$ (see Theorem 8.10), and also that $c_{1}(S)=0$ and $c_{2}(S)=24 \mathfrak{o}_{S}$. Then the lemma follows from spelling out the equation $(p \times p)_{*}(\rho \times q)^{*} \Delta_{\text {tot }}=0$ term by term using Lemma 14.2.

14.2. The Fourier transform for $S^{[2]}$. In the remaining part of this section, we assume that $X=S$ is a K3 surface so that $F=S^{[2]}$ is a hyperkähler fourfold. In that case, it is possible to refine the results of $\S 12$ and obtain a decomposition of the Chow groups of $F$, similar in every way to the Fourier decomposition we will eventually establish, by using the powers of the action of the incidence correspondence $I$. However, the incidence correspondence $I$ does not deform in moduli and the whole point of modifying $I$ into a cycle $L$ with cohomology class the Beauville-Bogomolov class $\mathfrak{B}$ is to have at our disposal a cycle that would deform in moduli (and in fact our cycle $L$ defined in (92) does deform as will be shown in §16) and whose deformations would induce a Fourier decomposition for all hyperkähler varieties of $\mathrm{K} 3^{[2]}$-type.

In concrete terms, the goal here is to prove Theorem 2 for $F$. For that purpose, we first construct a cycle $L \in \mathrm{CH}^{2}(F \times F)$ that represents the Beauville-Bogomolov class $\mathfrak{B}$, and we show (Theorem 14.5) that $L$ satisfies Conjecture 1, i.e., that $L^{2}=2 \Delta_{F}-\frac{2}{25}\left(l_{1}+l_{2}\right) \cdot L-\frac{1}{23 \cdot 25}\left(2 l_{1}^{2}-23 l_{1} l_{2}+2 l_{2}^{2}\right) \in \mathrm{CH}^{4}(F \times F)$. Then we check that Properties (7) and (8) are satisfied by $L$ (Proposition 14.6). We can thus apply Theorem 2.2. Finally, we show that $L$ satisfies Property (9) (Proposition 14.8) so that we can apply Theorem 2.4 and get the Fourier decomposition of Theorem 2 for $F$.

It is easy to see that $\Delta_{S}-p_{1}^{*} \mathfrak{o}_{S}-p_{2}^{*} \mathfrak{o}_{S}$ represents the intersection form on $S$. From the definition of $I$, a simple computation gives

$$
\begin{aligned}
(p \times p)_{*}(q \times q)^{*}\left(\Delta_{S}-p_{1}^{*} \mathfrak{o}_{S}-p_{2}^{*} \mathfrak{o}_{S}\right) & =I-(p \times p)_{*}\left(p_{1}^{*} q^{*} \mathfrak{o}_{S} \cdot p_{2}^{*} p^{*}[F]+p_{2}^{*} q^{*} \mathfrak{o}_{S} \cdot p_{1}^{*} p^{*}[F]\right) \\
& =I-2 p_{1}^{*} S_{\mathfrak{o}}-2 p_{2}^{*} S_{\mathfrak{o}} .
\end{aligned}
$$

Here the coefficient 2 appears in the second equality because $p: Z \rightarrow F$ is of degree 2 . As a result, the cycle

$$
L_{\delta}:=I-2 p_{1}^{*} S_{\mathfrak{o}}-2 p_{2}^{*} S_{\mathfrak{o}}
$$

represents the $\left(\mathrm{H}^{2}(S, \mathbb{Q}) \otimes \mathrm{H}^{2}(S, \mathbb{Q})\right)$-component of the Beauville-Bogomolov class $\mathfrak{B} \in \mathrm{H}^{2}(F, \mathbb{Q}) \otimes$ $\mathrm{H}^{2}(F, \mathbb{Q}) \subset \mathrm{H}^{4}(F \times F, \mathbb{Q})$ with respect to the $q_{F}$-orthogonal decomposition (56). Hence we see that

$$
L:=I-2 p_{1}^{*} S_{\mathfrak{o}}-2 p_{2}^{*} S_{\mathfrak{o}}-\frac{1}{2} \delta_{1} \delta_{2}
$$

represents the Beauville-Bogomolov class $\mathfrak{B}$, where $\delta_{i}=p_{i}^{*} \delta, i=1,2$. Proposition 11.7, together with the identity $c_{2}(S)=24 \mathfrak{o}_{S}$ of Beauville-Voisin [11], implies

$$
\left(\iota_{\Delta}\right)^{*} I=24 S_{\mathfrak{o}}-2 \delta^{2} .
$$

Then we have

$$
l:=\left(\iota_{\Delta}\right)^{*} L=20 S_{\mathfrak{o}}-\frac{5}{2} \delta^{2}=\frac{5}{6} c_{2}(F),
$$

where the last equality is the following lemma.

Lemma 14.4. If $S$ is a K3 surface, then $c_{2}\left(S^{[2]}\right)=p_{*} q^{*} c_{2}(S)-3 \delta^{2}=24 S_{\mathfrak{o}}-3 \delta^{2}$.

Proof. We take up the notations of diagram (54) with $X=S$. The blow-up morphism $\rho: Z \rightarrow S \times S$ gives a short exact sequence

$$
0 \longrightarrow \rho^{*} \Omega_{S \times S}^{1} \longrightarrow \Omega_{Z}^{1} \longrightarrow j_{*}^{\prime} \Omega_{E / S}^{1} \longrightarrow 0 .
$$

Note that $j^{\prime} \Omega_{E / S}^{1} \cong \mathcal{O}_{E}(2 E)$, and hence

$$
c\left(j_{*}^{\prime} \Omega_{E / S}^{1}\right)=\frac{1+2 E}{1+E}=1+E-E^{2}+E^{3}-E^{4} .
$$

By taking Chern classes of the sheaves in the above short exact sequence, we get

$$
c\left(\Omega_{Z}^{1}\right)=\rho^{*} c\left(\Omega_{S \times S}^{1}\right) \cdot c\left(j_{*}^{\prime} \Omega_{E / S}^{1}\right)=\rho^{*}\left(p_{1}^{*} c\left(\Omega_{S}^{1}\right) \cdot p_{2}^{*} c\left(\Omega_{S}^{1}\right)\right) \cdot \frac{1+2 E}{1+E} .
$$


Meanwhile the double cover $p: Z \rightarrow F$ gives the following short exact sequence

$$
0 \longrightarrow p^{*} \Omega_{F}^{1} \longrightarrow \Omega_{Z}^{1} \longrightarrow \mathcal{O}_{E}(-E) \longrightarrow 0 .
$$

By taking Chern classes, we get

$$
p^{*} c\left(\Omega_{F}^{1}\right)=c\left(\Omega_{Z}^{1}\right) / c\left(\mathcal{O}_{E}(-E)\right)=c\left(\Omega_{Z}^{1}\right) \cdot \frac{1-2 E}{1-E}
$$

Combining the above two computations yields

$$
p^{*} c\left(\Omega_{F}^{1}\right)=\rho^{*}\left(p_{1}^{*} c\left(\Omega_{S}^{1}\right) \cdot p_{2}^{*} c\left(\Omega_{S}^{1}\right)\right) \cdot \frac{1-4 E^{2}}{1-E^{2}}
$$

This gives $p^{*} c_{2}(F)=\rho^{*}\left(p_{1}^{*} c_{2}(S)+p_{2}^{*} c_{2}(S)\right)-3 E^{2}$. We apply $p_{*}$ to this equation and note that $p_{*} E^{2}=2 \delta^{2}$ and $p_{*} \rho^{*} p_{i}^{*} c_{2}(S)=p_{*} q^{*} c_{2}(S), i=1,2$. Then we get

$$
2 c_{2}(F)=2 p_{*} q^{*} c_{2}(S)-6 \delta^{2}
$$

which, in light of Beauville-Voisin's formula $c_{2}(S)=24 \mathfrak{o}_{S}$, proves the lemma.

Theorem 14.5. Conjecture 1 holds for $F$ with $L$ as in (92).

Proof. First note that Proposition 11.4 gives the equation

$$
I^{2}=2 \Delta_{F}-\left(\delta_{1}^{2}-\delta_{1} \delta_{2}+\delta_{2}^{2}\right) \cdot I+24 p_{1}^{*} S_{\mathfrak{o}} \cdot p_{2}^{*} S_{\mathfrak{o}} .
$$

If we substitute $I=L+2 p_{1}^{*} S_{\mathfrak{o}}+2 p_{2}^{*} S_{\mathfrak{o}}+\frac{1}{2} \delta_{1} \delta_{2}$ into the above equation, we get

$$
L^{2}=2 \Delta_{F}-\left(\delta_{1}^{2}+\delta_{2}^{2}+4 p_{1}^{*} S_{\mathfrak{o}}+4 p_{2}^{*} S_{\mathfrak{o}}\right) \cdot L+P_{1}\left(\delta_{1}, \delta_{2}, p_{1}^{*} S_{\mathfrak{o}}, p_{2}^{*} S_{\mathfrak{o}}\right),
$$

where $P_{1}$ is a weighted homogeneous polynomial of degree 4 . Lemma 14.3 implies that $\left(\delta_{1}^{2}+2 p_{1}^{*} S_{\mathfrak{o}}\right) \cdot L$ is a weighted homogeneous polynomial of degree 4 in $\left(\delta_{1}, \delta_{2}, p_{1}^{*} S_{\mathfrak{o}}, p_{2}^{*} S_{\mathfrak{o}}\right)$. By symmetry, so is $\left(\delta_{2}^{2}+2 p_{2}^{*} S_{\mathfrak{o}}\right) \cdot L$. From the equation (93), we get

$$
\frac{2}{25} l-\left(4 S_{\mathfrak{o}}+\delta^{2}\right)=-\frac{6}{5}\left(\delta^{2}+2 S_{\mathfrak{o}}\right) .
$$

Hence $p_{i}^{*}\left(\frac{2}{25} l-\left(4 S_{\mathfrak{o}}+\delta^{2}\right)\right) \cdot L, i=1,2$, is a weighted homogeneous polynomial of degree 4 in $\left(\delta_{1}, \delta_{2}, p_{1}^{*} S_{\mathfrak{o}}, p_{2}^{*} S_{\mathfrak{o}}\right)$. From equation (95), we get

$$
L^{2}=2 \Delta_{F}-\frac{2}{25}\left(l_{1}+l_{2}\right) \cdot L+P_{2}\left(\delta_{1}, \delta_{2}, p_{1}^{*} S_{\mathfrak{o}}, p_{2}^{*} S_{\mathfrak{o}}\right),
$$

where $P_{2}$ is a weighted homogeneous polynomial of degree 4 which is homologically equivalent to $\frac{-1}{23 \cdot 25}\left(2 l_{1}^{2}-\right.$ $\left.23 l_{1} l_{2}+2 l_{2}^{2}\right)$. Then Lemma 4.8 shows that $P_{2}=\frac{-1}{23 \cdot 25}\left(2 l_{1}^{2}-23 l_{1} l_{2}+2 l_{2}^{2}\right)$.

We now check that $L$ satisfies conditions (7) and (8) of Theorem 2.2 .

Proposition 14.6. We have $L_{*} l^{2}=0$ and $L_{*}\left(l \cdot L_{*} \sigma\right)=25 L_{*} \sigma$ for all $\sigma \in \mathrm{CH}_{0}(F)$.

Proof. A direct computation using (92) yields

$$
L_{*}[x, y]=I_{*}[x, y]-2 S_{\mathfrak{o}}=S_{x}+S_{y}-2 S_{\mathfrak{o}} .
$$

By (93), $l$ is proportional to $c_{2}(F)$ and it follows, thanks to [54], that $l^{2}$ is proportional to $\left[\mathfrak{o}_{S}, \mathfrak{o}_{S}\right]$. Thus (97) gives $L_{*} l^{2}=0$.

Let now $a$ and $b$ be rational numbers. An easy computation using $(97), S_{x} \cdot S_{y}=[x, y]$ and $\delta^{2} \cdot S_{x}=$ $-[x, x]$ yields

$$
L_{*}\left(\left(a S_{\mathfrak{o}}+b \delta^{2}\right) \cdot L_{*}[x, y]\right)=(a-2 b) L_{*}[x, y] .
$$

The identity (93) $l=20 S_{\mathfrak{o}}-\frac{5}{2} \delta^{2}$ then gives $L_{*}\left(l \cdot L_{*} \sigma\right)=25 L_{*} \sigma$ for all $\sigma \in \mathrm{CH}_{0}(F)$.

We then check that $L$ satisfies condition (9) of Theorem 2.4. Let us first compare the decompositions of $\mathrm{CH}_{0}(F)$ induced by $I$ and $L$.

Lemma 14.7. We have

$$
\begin{aligned}
& \operatorname{ker}\left\{L_{*}: \mathrm{CH}_{0}(F)_{\text {hom }} \rightarrow \mathrm{CH}_{2}(F)\right\}=\operatorname{ker}\left\{I_{*}: \mathrm{CH}_{0}(F) \rightarrow \mathrm{CH}_{2}(F)\right\} ; \\
& \operatorname{ker}\left\{\left(L^{2}\right)_{*}: \mathrm{CH}_{0}(F) \rightarrow \mathrm{CH}_{0}(F)\right\}=\operatorname{ker}\left\{\left(I^{2}-4 \Delta_{F}\right)_{*}: \mathrm{CH}_{0}(F) \rightarrow \mathrm{CH}_{0}(F)\right\} .
\end{aligned}
$$


Proof. The first equality follows from (97) and from $I_{*}\left[\mathfrak{o}_{S}, \mathfrak{o}_{S}\right]=2 S_{\mathfrak{o}} \neq 0 \in \mathrm{CH}_{2}(F)$. A straightforward computation involving Proposition 12.6, (93), (6) and (97) gives

$$
\left(L^{2}\right)_{*}[x, y]=2[x, y]-2\left[\mathfrak{o}_{S}, x\right]-2\left[\mathfrak{o}_{S}, y\right]+2\left[\mathfrak{o}_{S}, \mathfrak{o}_{S}\right] .
$$

Thus $L^{2}$ acts as zero on cycles of the form $[x, x]$. Proposition 12.2 implies that $\operatorname{ker}\left\{\left(I^{2}-4 \Delta_{F}\right)_{*}\right\} \subseteq$ $\operatorname{ker}\left\{\left(L^{2}\right)_{*}\right\}$. Consider now $\sigma \in \mathrm{CH}_{0}(F)$ such that $\left(L^{2}\right)_{*} \sigma=0$. Then by (6) we see that $\sigma$ is a linear combination of $l \cdot L_{*} \sigma$ and of $l^{2}$. Since $l$ is a linear combination of $\delta^{2}$ and $S_{\mathfrak{o}}$, the cycle $\sigma$ is a linear combination of cycles of the form $[\mathfrak{o}, x]$. By Proposition 12.7, we conclude that $\left(I^{2}\right)_{*} \sigma=4 \sigma$.

Proposition 14.8. $\left(L^{2}\right)_{*}\left(l \cdot\left(L^{2}\right)_{*} \sigma\right)=0$ for all $\sigma \in \mathrm{CH}^{2}(F)$.

Proof. In view of Theorem 14.5 and Proposition 14.6, Theorem 2.2 gives eigenspace decompositions $\mathrm{CH}^{4}(F)=\Lambda_{0}^{4} \oplus \Lambda_{2}^{4}$ and $\mathrm{CH}^{2}(F)=\Lambda_{25}^{2} \oplus \Lambda_{2}^{2} \oplus \Lambda_{0}^{2}$ for the action of $L^{2}$. Moreover, $\Lambda_{25}^{2}=\langle l\rangle$ and Lemma 2.3 gives $\left(L^{2}\right)_{*} l^{2}=0$. Therefore, it is sufficient to prove that $l \cdot \sigma=0$ for all $\sigma \in \Lambda_{2}^{2}$. According to (37), $\sigma \in \Lambda_{2}^{2}$ if and only if $l \cdot \sigma \in \Lambda_{2}^{4}$. But, since (93) $l=20 S_{\mathfrak{o}}-\frac{5}{2} \delta^{2}$, Propositions 12.2 and 12.7, together with Lemma 14.7, show that $l \cdot \sigma \in \Lambda_{0}^{4}$ for all $\sigma \in \mathrm{CH}^{2}(F)$. Hence if $\sigma \in \Lambda_{2}^{2}$, then $l \cdot \sigma \in \Lambda_{0}^{4} \cap \Lambda_{2}^{4}=\{0\}$.

By the work carried out in Part I, the Fourier decomposition as in Theorem 2 is proved for the Hilbert scheme of length-2 subschemes of K3 surfaces.

\section{The Fourier DECOMPosition FOR $S^{[2]}$ IS MUltiplicAtive}

Let $F=S^{[2]}$ be the Hilbert scheme of length-2 subschemes on a $K 3$ surface $S$. To avoid confusion, the Fourier decomposition of the Chow groups of $S^{[2]}$ obtained in Section 14.2 will be denoted $\mathrm{CH}_{\mathcal{F}}^{p}(-)_{s}$. We continue to denote $\mathrm{CH}_{\mathrm{CK}}^{p}(-)_{s}$ the decomposition induced by the multiplicative Chow-Künneth decomposition of Theorem 13.4 obtained by considering the multiplicative Chow-Künneth decomposition on $S$ of Example 8.17. The goal of this section is to show that these two decompositions coincide, thereby proving Theorem 3 in the case $F=S^{[2]}$.

15.1. The Chow-Künneth decomposition of $\mathrm{CH}^{*}\left(S^{[2]}\right)$. Let $S$ be a K3 surface and let $\mathfrak{o}_{S}$ be the class of a point on $S$ lying on a rational curve. Recall that the following idempotents in $\mathrm{CH}^{2}(S \times S)$

$$
\pi_{S}^{0}=\mathfrak{o}_{S} \times S, \quad \pi_{S}^{4}=S \times \mathfrak{o}_{S}, \quad \pi_{S}^{2}=\Delta_{S}-\pi_{S}^{0}-\pi_{S}^{4}
$$

define a multiplicative Chow-Künneth decomposition of $S$ in the sense of Definition 8.1. The Chow ring of $S$ inherits a bigrading

$$
\mathrm{CH}^{i}(S)_{s}:=\left(\pi_{S}^{2 i-s}\right)_{*} \mathrm{CH}^{i}(S) .
$$

As explained in the introduction, this bigrading coincides with the one induced by the Fourier transform $\mathcal{F}(-):=\left(p_{2}\right)_{*}\left(\pi_{S}^{2} \cdot p_{1}^{*}-\right)$. We will thus omit the subscript "CK" or " $\mathcal{F}$ " when mentioning the Chow ring of $S$.

The variety $S \times S$ is naturally endowed with the product Chow-Künneth decomposition given by

$$
\pi_{S \times S}^{k}=\sum_{i+j=k} \pi_{S}^{i} \otimes \pi_{S}^{j}, \quad 0 \leq k \leq 8 .
$$

According to Theorem 8.6, this Chow-Künneth decomposition is multiplicative and induces a bigrading

$$
\mathrm{CH}_{\mathrm{CK}}^{i}(S \times S)_{s}:=\left(\pi_{S \times S}^{2 i-s}\right)_{*} \mathrm{CH}^{i}(S \times S)
$$

of the Chow ring of $S \times S$.

Let $p_{i}: S \times S \rightarrow S, i=1,2$, be the two projections. We write $(\mathfrak{o}, \mathfrak{o}):=p_{1}^{*} \mathfrak{o}_{S} \cdot p_{2}^{*} \mathfrak{o}_{S} \in \mathrm{CH}_{\mathrm{CK}}^{4}(S \times S)$. We define

$$
\begin{aligned}
\phi_{1}: S \rightarrow S \times S, & x \mapsto\left(x, \mathfrak{o}_{S}\right) ; \\
\phi_{2}: S \rightarrow S \times S, & x \mapsto\left(\mathfrak{o}_{S}, x\right) .
\end{aligned}
$$

Lemma 15.1. The above morphisms $\phi_{i}, i=1,2$, satisfy

$$
\phi_{i}^{*} \mathrm{CH}_{\mathrm{CK}}^{p}(S \times S)_{s} \subseteq \mathrm{CH}^{p}(S)_{s} \quad \text { and } \quad \phi_{i_{*}} \mathrm{CH}^{p}(S)_{s} \subseteq \mathrm{CH}_{\mathrm{CK}}^{p+2}(S \times S)_{s} .
$$


Proof. By Proposition 8.7(i), we see that $\phi_{1_{*}}[S]=p_{2}^{*} \mathfrak{o}_{S}$ belongs to $\mathrm{CH}_{\mathrm{CK}}^{2}(S \times S)_{0}$. Let $\alpha \in \mathrm{CH}^{p}(S)_{s}$; then

$$
\phi_{1_{*}} \alpha=p_{1}^{*} \alpha \cdot \phi_{1_{*}} S \in \mathrm{CH}_{\mathrm{CK}}^{p}(S \times S)_{s} \cdot \mathrm{CH}_{\mathrm{CK}}^{2}(S \times S)_{0} \subseteq \mathrm{CH}_{\mathrm{CK}}^{p+2}(S \times S)_{s} .
$$

Let $\beta \in \mathrm{CH}_{\mathrm{CK}}^{p}(S \times S)_{s}$; then

$$
\phi_{1_{*}} \phi_{1}^{*} \beta=\beta \cdot \phi_{1_{*}} S \in \mathrm{CH}_{\mathrm{CK}}^{p+2}(S \times S)_{2} .
$$

Given that $\phi_{1_{*}}$ is injective and respects the Chow-Künneth decomposition, the above equation implies $\phi_{1}^{*} \beta \in \mathrm{CH}^{p}(S)_{s}$.

Proposition 15.2. The Chow-Künneth decomposition of the Chow groups of $S \times S$ can be described as

$$
\begin{aligned}
& \mathrm{CH}_{\mathrm{CK}}^{2}(S \times S)_{0}=\left\{\alpha \in \mathrm{CH}^{2}(S \times S): \alpha \cdot p_{1}^{*} \mathfrak{o}_{S} \in\langle(\mathfrak{o}, \mathfrak{o})\rangle, \alpha \cdot p_{2}^{*} \mathfrak{o}_{S} \in\langle(\mathfrak{o}, \mathfrak{o})\rangle\right\} \\
& \mathrm{CH}_{\mathrm{CK}}^{2}(S \times S)_{2}=p_{1}^{*} \mathrm{CH}^{2}(S)_{2} \oplus p_{2}^{*} \mathrm{CH}^{2}(S)_{2} \\
& \mathrm{CH}_{\mathrm{CK}}^{3}(S \times S)_{0}=p_{1}^{*} \mathfrak{o}_{S} \cdot p_{2}^{*} \mathrm{CH}^{1}(S) \oplus p_{1}^{*} \mathrm{CH}^{1}(S) \cdot p_{2}^{*} \mathfrak{o}_{S} \\
& \mathrm{CH}_{\mathrm{CK}}^{3}(S \times S)_{2}=\mathrm{CH}^{3}(S \times S)_{\mathrm{hom}} \\
& \mathrm{CH}_{\mathrm{CK}}^{4}(S \times S)_{0}=\mathbb{Q}(\mathfrak{o}, \mathfrak{o}) \\
& \mathrm{CH}_{\mathrm{CK}}^{4}(S \times S)_{2}=p_{1}^{*} \mathfrak{o}_{S} \cdot p_{2}^{*} \mathrm{CH}^{2}(S)_{2} \oplus p_{1}^{*} \mathrm{CH}^{2}(S)_{2} \cdot p_{2}^{*} \mathfrak{o}_{S} \\
& \mathrm{CH}_{\mathrm{CK}}^{4}(S \times S)_{4}=p_{1}^{*} \mathrm{CH}^{2}(S)_{2} \cdot p_{2}^{*} \mathrm{CH}^{2}(S)_{2}
\end{aligned}
$$

Proof. In this proof, we use $p_{S^{4}, i}$ and $p_{S^{4}, i j}$ to denote the projections from $S^{4}$ to the corresponding factor(s) and we use $p_{i}$ to denote the projection from $S^{2}$ to the corresponding factor.

Let $\alpha \in \mathrm{CH}^{2}(S \times S)$. Using the expression (99) for $\pi_{S \times S}^{k}$, we have

$$
\begin{aligned}
\left(\pi_{S \times S}^{0}\right)_{*} \alpha= & ((\mathfrak{o}, \mathfrak{o}) \times S \times S)_{*} \alpha=0 . \\
\left(\pi_{S \times S}^{2}\right)_{*} \alpha= & \left(\pi_{S}^{0} \otimes \pi_{S}^{2}\right)_{*} \alpha+\left(\pi_{S}^{2} \otimes \pi_{S}^{0}\right)_{*} \alpha \\
= & \left(p_{S^{4}, 34}\right)_{*}\left(p_{S^{4}, 1}^{*} \mathfrak{o}_{S} \cdot p_{S^{4}, 24}^{*} \pi_{S}^{2} \cdot p_{S^{4}, 12}^{*} \alpha\right) \\
& +\left(p_{S^{4}, 34}\right)_{*}\left(p_{S^{4}, 2}^{*} \mathfrak{o}_{S} \cdot p_{S^{4}, 13}^{*} \pi_{S}^{2} \cdot p_{S^{4}, 12}^{*} \alpha\right) \\
= & p_{2}^{*}\left(\pi_{S}^{2}\right)_{*} \phi_{2}^{*} \alpha+p_{1}^{*}\left(\pi_{S}^{2}\right)_{*} \phi_{1}^{*} \alpha . \\
\left(\pi_{S \times S}^{6}\right)_{*} \alpha= & 0 . \\
\left(\pi_{S \times S}^{8}\right)_{*} \alpha= & 0 .
\end{aligned}
$$

Note that $\alpha \cdot p_{i}^{*} \mathfrak{o}_{S} \in\langle(\mathfrak{o}, \mathfrak{o})\rangle$ if and only if $\phi_{j}^{*} \alpha \in\left\langle\mathfrak{o}_{S}\right\rangle$ for $(i, j)=(1,2)$ and $(i, j)=(2,1)$. If follows that if $\alpha \cdot p_{i}^{*} \mathfrak{o}_{S} \in\langle(\mathfrak{o}, \mathfrak{o})\rangle$, then $\left(\pi_{S \times S}^{k}\right)_{*} \alpha=0$ for all $k \neq 4$. Therefore $\alpha$ belongs to $\mathrm{CH}_{\mathrm{CK}}^{2}(S \times S)_{0}$. Conversely, if $\alpha \in \mathrm{CH}_{\mathrm{CK}}^{2}(S \times S)_{0}$, then $\alpha \cdot p_{i}^{*} \mathfrak{o}_{S} \in \mathrm{CH}_{\mathrm{CK}}^{4}(S \times S)_{0}=\langle(\mathfrak{o}, \mathfrak{o})\rangle$. This proves the first equation. The remaining equations are easier to establish and their proofs are thus omitted.

Let $\rho: Z \rightarrow S \times S$ be the blow-up of $S \times S$ along the diagonal. We have the following commutative diagram

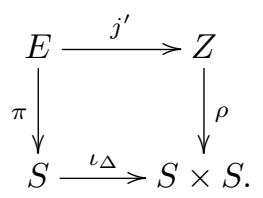

The K3 surface $S$ is endowed with its multiplicative self-dual Chow-Künneth decomposition (98). We also have $c_{1}(S)=0$, and $c_{2}(S)=24 \mathrm{o}_{S}$ which is in $\mathrm{CH}^{2}(S)_{0}$. Therefore Proposition 13.3 yields the existence of a multiplicative Chow-Künneth decomposition for $Z$ inducing a bigrading

$$
\mathrm{CH}^{i}(Z)_{s}=\rho^{*} \mathrm{CH}^{i}(S \times S)_{s} \oplus j_{*}^{\prime} \pi^{*} \mathrm{CH}^{i-1}(S)_{s}
$$

of the Chow ring of $Z$. 
Proposition 15.3. The Chow-Künneth decomposition (100) of the Chow groups of $Z$ have the following description

$$
\begin{aligned}
& \mathrm{CH}_{\mathrm{CK}}^{2}(Z)_{0}=\left\{\alpha \in \mathrm{CH}^{2}(Z): \alpha \cdot \rho^{*} p_{i}^{*} \mathfrak{o}_{S} \in\left\langle\mathfrak{o}_{Z}\right\rangle, \forall i=1,2\right\} ; \\
& \mathrm{CH}_{\mathrm{CK}}^{2}(Z)_{2}=\rho^{*} \mathrm{CH}_{\mathrm{CK}}^{2}(S \times S)_{2} ; \\
& \mathrm{CH}_{\mathrm{CK}}^{3}(Z)_{0}=\rho^{*} \mathrm{CH}_{\mathrm{CK}}^{3}(S \times S)_{0} \oplus \mathbb{Q} \cdot j_{*}^{\prime} \pi^{*} \mathfrak{o}_{S} ; \\
& \mathrm{CH}_{\mathrm{CK}}^{3}(Z)_{2}=\mathrm{CH}^{3}(Z)_{\mathrm{hom}} \\
& \mathrm{CH}_{\mathrm{CK}}^{4}(Z)_{0}=\left\langle\mathfrak{o}_{Z}\right\rangle \\
& \mathrm{CH}_{\mathrm{CK}}^{4}(Z)_{2}=\rho^{*} \mathrm{CH}_{\mathrm{CK}}^{4}(S \times S)_{2} ; \\
& \mathrm{CH}_{\mathrm{CK}}^{4}(Z)_{4}=\rho^{*} \mathrm{CH}_{\mathrm{CK}}^{4}(S \times S)_{4} ;
\end{aligned}
$$

where $\mathfrak{o}_{Z}:=\rho^{*}(\mathfrak{o}, \mathfrak{o})$.

Proof. This follows from formula (100) and Proposition 15.2. For example, consider $\alpha \in \mathrm{CH}^{2}(Z)$. We can write

$$
\alpha=\rho^{*} \alpha^{\prime}+j_{*}^{\prime} \pi^{*} \beta,
$$

for some $\alpha^{\prime} \in \mathrm{CH}^{2}(S \times S)$ and some $\beta \in \mathrm{CH}^{1}(S)$. Then

$$
\begin{aligned}
\alpha \cdot \rho^{*} p_{1}^{*} \mathfrak{o}_{S} & =\rho^{*}\left(\alpha^{\prime} \cdot p_{1}^{*} \mathfrak{o}_{S}\right)+j_{*}^{\prime}\left(\pi^{*} \beta \cdot j^{\prime *} \rho^{*} p_{1}^{*} \mathfrak{o}_{S}\right) \\
& =\rho^{*}\left(\alpha^{\prime} \cdot p_{1}^{*} \mathfrak{o}_{S}\right)+j_{*}^{\prime}\left(\pi^{*} \beta \cdot \pi^{*} \iota_{\Delta}^{*} p_{1}^{*} \mathfrak{o}_{S}\right) \\
& =\rho^{*}\left(\alpha^{\prime} \cdot p_{1}^{*} \mathfrak{o}_{S}\right)+j_{*}^{\prime}\left(\pi^{*} \beta \cdot \pi^{*} \mathfrak{o}_{S}\right) \\
& =\rho^{*}\left(\alpha^{\prime} \cdot p_{1}^{*} \mathfrak{o}_{S}\right) .
\end{aligned}
$$

Hence the condition $\alpha \cdot \rho^{*} p_{1}^{*} \mathfrak{o}_{S} \in\left\langle\mathfrak{o}_{Z}\right\rangle$ is equivalent to $\alpha^{\prime} \cdot p_{1}^{*} \mathfrak{o}_{S} \in\langle(\mathfrak{o}, \mathfrak{o})\rangle$, which is further equivalent to $\alpha^{\prime} \in \mathrm{CH}_{\mathrm{CK}}^{2}(S \times S)_{0}$. By symmetry, the same holds if we replace $p_{1}$ by $p_{2}$. This yields the first equality. All the other equalities can be verified similarly.

15.2. The Fourier decomposition of $\mathrm{CH}^{*}\left(S^{[2]}\right)$. We now provide an explicit description of the Fourier decomposition on the Chow groups of $F$. A satisfying description consists in understanding how $S_{\mathfrak{o}}$ and $\delta$ intersect with 2-cycles. For that matter, Lemma 15.5 is crucial ; it relies on the key Lemma 14.3.

Let $p: Z \rightarrow F=S^{[2]}$ be the natural double cover and $j=p \circ j^{\prime}: E \rightarrow F$. Recall the following definition (72) from Section 12 :

$$
\mathcal{A}:=I_{*} \mathrm{CH}^{4}(F) \subset \mathrm{CH}^{2}(F) .
$$

Note that $\mathcal{A}$ is generated by cycles of the form $S_{x}, x \in S$.

Lemma 15.4. The Fourier decomposition enjoys the following properties.

(i) $\mathrm{CH}_{\mathcal{F}}^{2}(F)_{2}=\mathcal{A}_{\text {hom }}$;

(ii) $\mathrm{CH}_{\mathcal{F}}^{4}(F)_{2}=\langle[\mathfrak{o}, x]-[\mathfrak{o}, y]\rangle, x, y \in S$;

(iii) $I_{*}: \mathrm{CH}_{\mathcal{F}}^{4}(F)_{2} \rightarrow \mathrm{CH}_{\mathcal{F}}^{2}(F)_{2}$ is an isomorphism and its inverse is given by intersecting with $S_{\mathfrak{o}}$;

(iv) $\delta \cdot \mathrm{CH}^{3}(F) \subseteq \mathrm{CH}^{4}(F)_{2} \oplus \mathrm{CH}^{4}(F)_{0}$.

Proof. By Theorems 2.2 and 2.4 and equation (97), we have

$$
\mathrm{CH}_{\mathcal{F}}^{2}(F)_{2}=\Lambda_{0}^{2}=L_{*} \mathrm{CH}^{4}(F)=\mathcal{A}_{\text {hom }} .
$$

This proves (i). For (ii), we argue as follows

$$
\begin{aligned}
\mathrm{CH}_{\mathcal{F}}^{4}(F)_{2} & =\left(\Lambda_{0}^{4}\right)_{\text {hom }} \quad(\text { Theorem 2.4) } \\
& =\left(\operatorname{ker}\left\{L^{2}: \mathrm{CH}^{4}(F) \rightarrow \mathrm{CH}^{4}(F)\right\}\right)_{\text {hom } \left.\quad \text { (by definition of } \Lambda_{0}^{4}\right)} \\
& =\left(\operatorname{ker}\left\{I^{2}-4 \Delta_{F}: \mathrm{CH}^{4}(F) \rightarrow \mathrm{CH}^{4}(F)\right\}\right)_{\text {hom }} \quad \text { (Lemma 14.7) } \\
& =\operatorname{im}\left\{\mathrm{CH}_{0}\left(S_{\mathfrak{o}}\right)_{\text {hom }} \rightarrow \mathrm{CH}_{0}(F)\right\} \quad \text { (Proposition 12.7). }
\end{aligned}
$$


Statement (iii) follows from (i) and (ii). Note that Proposition 12.6 shows that a 0-cycle supported on $E$ is also supported on $S_{\mathfrak{o}}$. Hence any 0-cycle supported on $E$ belongs to $\mathrm{CH}_{\mathcal{F}}^{4}(F)_{0} \oplus \mathrm{CH}_{\mathcal{F}}^{4}(F)_{2}$. Statement (iv) follows since $2 \delta=j_{*} E$.

We now come to the crucial lemma.

Lemma 15.5. Let $\alpha \in \mathrm{CH}_{\mathcal{F}}^{2}(F)_{0}$, then

$$
\alpha \cdot S_{\mathfrak{o}} \in \mathrm{CH}_{\mathcal{F}}^{4}(F)_{0} \quad \text { and } \quad \alpha \cdot \delta^{2} \in \mathrm{CH}_{\mathcal{F}}^{4}(F)_{0} .
$$

Proof. On the one hand, by Proposition 4.2 and equation (93), we know that

$$
\alpha \cdot l=\alpha \cdot\left(20 S_{\mathfrak{o}}+\frac{5}{2} \delta^{2}\right)=20 \alpha \cdot S_{\mathfrak{o}}+\frac{5}{2} \alpha \cdot \delta^{2} \in \mathrm{CH}_{\mathcal{F}}^{4}(F)_{0} .
$$

On the other hand, by the key Lemma 14.3, we see that

$$
I_{*}\left(2 \alpha \cdot S_{\mathfrak{o}}+\alpha \cdot \delta^{2}\right)=\left(p_{1}^{*}\left(2 S_{\mathfrak{o}}+\delta^{2}\right) \cdot I\right)_{*} \alpha \quad \text { is a multiple of } S_{\mathfrak{o}} \text { in } \mathrm{CH}^{2}(F) .
$$

Since $\alpha \cdot S_{\mathfrak{o}}$ is supported on $S_{\mathfrak{o}}$ and since $\alpha \cdot \delta^{2}$ is supported on the boundary divisor, it follows from Lemma 15.4 that

$$
\beta:=2 \alpha \cdot S_{\mathfrak{o}}+\alpha \cdot \delta^{2} \in \mathrm{CH}_{\mathcal{F}}^{4}(F)_{0} \oplus \mathrm{CH}_{\mathcal{F}}^{0}(F)_{2} .
$$

Note that $S_{\mathfrak{o}}$ is a linear combination of $l$ and $\delta^{2}$. We know that $l \in \mathrm{CH}_{\mathcal{F}}^{2}(F)_{0}$ (Theorem 2.4) and $\delta^{2} \in$ $\mathrm{CH}_{\mathcal{F}}^{2}(F)_{0}$ (Theorem 4.6). It follows that $S_{\mathfrak{o}} \in \mathrm{CH}_{\mathcal{F}}^{2}(F)_{0}$. By Lemma 15.4, $I_{*}: \mathrm{CH}_{\mathcal{F}}^{4}(F)_{2} \rightarrow \mathrm{CH}_{\mathcal{F}}^{2}(F)_{2}$ is an isomorphism. Hence the fact that $I_{*} \beta$ is a multiple of $S_{\mathfrak{o}} \in \mathrm{CH}_{\mathcal{F}}^{2}(F)_{0}$ implies that $\beta \in \mathrm{CH}_{\mathcal{F}}^{4}(F)_{0}$. Thus we have two different linear combinations of $S_{\mathfrak{o}}$ and $\delta^{2}$ whose intersections with $\alpha$ are multiples of $\mathfrak{o}_{F}$. The lemma follows immediately.

In order to get a description of the Fourier decomposition on $F$, we introduce some new notations. For a divisor $D \in \mathrm{CH}^{1}(S)$, we write

$$
\begin{aligned}
\hat{D} & :=p_{*} \rho^{*} p_{1}^{*} D \in \mathrm{CH}^{1}(F) ; \\
D_{\mathfrak{o}} & :=S_{\mathfrak{o}} \cdot \hat{D} .
\end{aligned}
$$

Proposition 15.6. The Fourier decomposition enjoys the following properties.

$$
\begin{aligned}
\mathrm{CH}_{\mathcal{F}}^{2}(F)_{0} & =\left\{\alpha \in \mathrm{CH}^{2}(F): \alpha \cdot S_{\mathfrak{o}} \in\left\langle\mathfrak{o}_{F}\right\rangle\right\} \\
& =\left\{\alpha \in \mathrm{CH}^{2}(F): \alpha \cdot \delta^{2} \in\left\langle\mathfrak{o}_{F}\right\rangle\right\} ; \\
\mathrm{CH}_{\mathcal{F}}^{2}(F)_{2} & =\left\langle S_{x}-S_{y}\right\rangle, \quad x, y \in S ; \\
\mathrm{CH}_{\mathcal{F}}^{3}(F)_{0} & =j_{*} \pi^{*} \mathfrak{o}_{S} \oplus\left\{D_{\mathfrak{o}}: D \in \mathrm{CH}^{1}(S)\right\} ; \\
\mathrm{CH}_{\mathcal{F}}^{3}(F)_{2} & =\mathrm{CH}^{3}(F)_{\mathrm{hom}} ; \\
\mathrm{CH}_{\mathcal{F}}^{4}(F)_{0} & =\left\langle\mathfrak{o}_{F}\right\rangle ; \\
\mathrm{CH}_{\mathcal{F}}^{4}(F)_{2} & =\langle[\mathfrak{o}, x]-[\mathfrak{o}, y]\rangle, \quad x, y \in S ; \\
\mathrm{CH}_{\mathcal{F}}^{4}(F)_{4} & =\mathrm{CH}_{\mathcal{F}}^{2}(F)_{2} \cdot \mathrm{CH}_{\mathcal{F}}^{2}(F)_{2} .
\end{aligned}
$$

Proof. If $\alpha \in \mathrm{CH}_{\mathcal{F}}^{2}(F)_{0}$, then by Lemma 15.5 we know that both of $\alpha \cdot S_{\mathfrak{o}}$ and $\alpha \cdot \delta^{2}$ are multiples of $\mathfrak{o}_{F}$. Conversely, let $\alpha \in \mathrm{CH}^{2}(F)$ be an arbitrary 2-cycle on $F$. Then we can write

$$
\alpha=\alpha_{0}+\alpha_{2}, \quad \alpha_{0} \in \mathrm{CH}_{\mathcal{F}}^{2}(F)_{0}, \quad \alpha_{2} \in \mathrm{CH}_{\mathcal{F}}^{2}(F)_{2} .
$$

Assume that $\alpha \cdot S_{\mathfrak{o}} \in \mathrm{CH}_{\mathcal{F}}^{4}(F)_{0}$. Note that Lemma 15.5 implies that $\alpha_{0} \cdot S_{\mathfrak{o}} \in \mathrm{CH}_{\mathcal{F}}^{4}(F)_{0}$. Hence we get $\alpha_{2} \cdot S_{\mathfrak{o}} \in \mathrm{CH}_{\mathcal{F}}^{4}(F)_{0}$. Since intersecting with $S_{\mathfrak{o}}$ defines an isomorphism between $\mathrm{CH}_{\mathcal{F}}^{2}(F)_{2}$ and $\mathrm{CH}_{\mathcal{F}}^{4}(F)_{2}$ by Lemma 15.4 (iii), it follows that $\alpha_{2} \cdot S_{\mathfrak{o}}=0$. Therefore

$$
\alpha_{2}=I_{*}\left(\alpha_{2} \cdot S_{\mathfrak{o}}\right)=0 .
$$


Thus $\alpha=\alpha_{0} \in \mathrm{CH}_{\mathcal{F}}^{2}(F)_{0}$. This establishes the first equality. The second equality is established similarly. The equations involving $\mathrm{CH}_{\mathcal{F}}^{2}(F)_{2}$ and $\mathrm{CH}_{\mathcal{F}}^{4}(F)_{2}$ are direct consequences of Lemma 15.4. Combining Theorems 2.2 and 2.4, we have

$$
\mathrm{CH}_{\mathcal{F}}^{3}(F)_{2}=\Lambda_{2}^{3}=\mathrm{CH}^{3}(F)_{\text {hom }} .
$$

The equation involving $\mathrm{CH}_{\mathcal{F}}^{3}(F)_{0}$ follows from the identity $\mathrm{CH}_{\mathcal{F}}^{3}(F)_{0}=\mathrm{CH}^{1}(F)^{\cdot 3}$ of Remark 4.7. Indeed, we have $\mathrm{CH}^{1}(F)=\langle\delta\rangle \oplus\left\{\hat{D}: D \in \mathrm{CH}^{1}(S)\right\}$. A direct calculation gives

$$
\begin{aligned}
\delta^{3} & =-12 j_{*} \pi^{*} \mathfrak{o}_{S} ; \\
\delta^{2} \cdot \hat{D} & =-j_{*}\left(\xi \cdot \pi^{*} D\right) ; \\
\delta \cdot \hat{D}_{1} \cdot \hat{D}_{2} & =2 j_{*} \pi^{*}\left(D_{1} \cdot D_{2}\right) ; \\
\hat{D}_{1} \cdot \hat{D}_{2} \cdot \hat{D}_{3} & =a_{1}\left(D_{1}\right)_{\mathfrak{o}}+a_{2}\left(D_{2}\right)_{\mathfrak{o}}+a_{3}\left(D_{3}\right)_{\mathfrak{o}} ;
\end{aligned}
$$

where $\xi \in \mathrm{CH}^{1}(E)$ is the class of the relative $\mathcal{O}(1)$ bundles and the $a_{i}$ 's are rational numbers. Note that

$$
p^{*} j_{*}\left(\xi \cdot \pi^{*} D\right)=2 j_{*}^{\prime}\left(\xi \cdot \pi^{*} D\right) \in \mathrm{CH}_{\mathrm{CK}}^{3}(Z)_{0} .
$$

By Propositions 15.2 and 15.3, we get

$$
p^{*} j_{*}\left(\xi \cdot \pi^{*} D\right)=\rho^{*}\left(D_{1}^{\prime} \times \mathfrak{o}_{S}+\mathfrak{o}_{S} \times D_{2}^{\prime}\right)+b j_{*}^{\prime} \pi^{*} \mathfrak{o}_{S}, \quad b \in \mathbb{Q} .
$$

Applying $p_{*}$ to the above we find

$$
j_{*}\left(\xi \cdot \pi^{*} D\right)=\frac{1}{2}\left(\left(D_{1}^{\prime}\right)_{\mathfrak{o}}+\left(D_{2}^{\prime}\right)_{\mathfrak{o}}\right)+\frac{1}{2} j_{*} \pi^{*} \mathfrak{o}_{S} .
$$

Combining all the above equations, we get the expression for $\mathrm{CH}_{\mathcal{F}}^{3}(F)_{0}$.

It remains to show the equation involving $\mathrm{CH}_{\mathcal{F}}^{4}(F)_{4}$. This can be done as follows

$$
\begin{aligned}
\mathrm{CH}_{\mathcal{F}}^{4}(F)_{4} & =\Lambda_{2}^{4} \quad(\text { Theorem 2.4) } \\
& =\operatorname{ker}\left\{L_{*}: \mathrm{CH}^{4}(F)_{\text {hom } \left.\rightarrow \mathrm{CH}^{2}(F)\right\} \quad \text { (Theorem 2.2) }}\right. \\
& =\operatorname{ker}\left\{I_{*}: \mathrm{CH}^{4}(F) \rightarrow \mathrm{CH}^{2}(F)\right\} \quad(\text { Lemma 14.7) } \\
& =\mathcal{A}_{\text {hom }} \cdot \mathcal{A}_{\text {hom } \quad(\text { Proposition 12.9) }} \\
& =\mathrm{CH}_{\mathcal{F}}^{2}(F)_{2} \cdot \mathrm{CH}_{\mathcal{F}}^{2}(F)_{2},
\end{aligned}
$$

where the last equality follows from Lemma 15.4.

15.3. The Chow-Künneth decomposition and the Fourier decomposition agree. Recall that $p: Z \rightarrow F$ denotes the quotient map from the blow-up of $X \times X$ along the diagonal to the Hilbert scheme $F=X^{[2]}$. The following proposition shows that the Chow-Künneth decomposition $(100)$ on $\mathrm{CH}^{*}(Z)$ is compatible with the Fourier decomposition of Theorem 2 on $\mathrm{CH}^{*}(F)$, via the morphism $p$.

Proposition 15.7. The following holds

$$
\mathrm{CH}_{\mathcal{F}}^{i}(F)_{s}=\left\{\alpha \in \mathrm{CH}^{i}(F): p^{*} \alpha \in \mathrm{CH}_{\mathrm{CK}}^{i}(Z)_{s}\right\} .
$$

Proof. This is a direct consequence of Propositions 15.2, 15.3 and 15.6. For example, consider a 2-cycle $\alpha \in \mathrm{CH}^{2}(F)$. If $p^{*} \alpha \in \mathrm{CH}_{\mathrm{CK}}^{2}(F)_{0}$, then

$$
p^{*} \alpha \cdot \rho^{*} p_{1}^{*} \mathfrak{o}_{S}=a \mathfrak{o}_{Z}, \quad \text { for some } a \in \mathbb{Q} .
$$

We apply $p_{*}$ to the above equation and find

$$
\alpha \cdot S_{\mathfrak{o}}=p_{*}\left(p^{*} \alpha \cdot \rho^{*} p_{1}^{*} \mathfrak{o}_{S}\right)=a p_{*} \mathfrak{o}_{Z}=a \mathfrak{o}_{F} .
$$

This yields that $\alpha \in \mathrm{CH}_{\mathcal{F}}^{2}(F)_{0}$. Conversely, if $\alpha \in \mathrm{CH}_{\mathcal{F}}^{2}(F)_{0}$, then we write

$$
p^{*} \alpha=\beta_{0}+\beta_{2}, \quad \beta_{s} \in \mathrm{CH}_{\mathrm{CK}}^{2}(Z)_{s} .
$$

The condition $\alpha \cdot S_{\mathfrak{o}}=b_{\mathfrak{o}_{F}}$ for some $b \in \mathbb{Q}$ implies that

$$
p_{*}\left(p^{*} \alpha \cdot \rho^{*} p_{i}^{*} \mathfrak{o}_{S}\right)=\alpha \cdot S_{\mathfrak{o}}=b \mathfrak{o}_{F} .
$$


It follows that $p_{*}\left(\beta_{2} \cdot \rho^{*} p_{i}^{*} \mathfrak{o}_{S}\right)=b^{\prime} \mathfrak{o}_{F}$. Since $\beta_{2}$ is homologically trivial, we actually have

$$
p_{*}\left(\beta_{2} \cdot \rho^{*} p_{i}^{*} \mathfrak{o}_{S}\right)=0 .
$$

Since $\beta_{2}$ is symmetric, we can write

$$
\beta_{2}=\sum_{i} \rho^{*}\left(p_{1}^{*}\left(x_{i}-y_{i}\right)+p_{2}^{*}\left(x_{i}-y_{i}\right)\right), \quad \text { for some } x_{i}, y_{i} \in S .
$$

Therefore we have

$$
0=p_{*}\left(\beta_{2} \cdot \rho^{*} p_{1}^{*} \mathfrak{o}_{S}\right)=p_{*} \rho^{*}\left(\sum_{i}\left(\mathfrak{o}, x_{i}\right)-\left(\mathfrak{o}, y_{i}\right)\right)=\sum_{i}\left(\left[\mathfrak{o}, x_{i}\right]-\left[\mathfrak{o}, y_{i}\right]\right) .
$$

It follows that $\sum_{i}\left(x_{i}-y_{i}\right)=0$ in $\mathrm{CH}^{2}(S)$. Thus we get $\beta_{2}=0$, namely $p^{*} \alpha \in \mathrm{CH}_{\mathrm{CK}}^{2}(Z)_{0}$. All the other cases can be easily proved and the details are thus omitted.

Given Remark 13.5, we readily get the following

Theorem 15.8. The Fourier decomposition agrees with the Chow-Künneth decomposition, namely

$$
\mathrm{CH}_{\mathcal{F}}^{p}\left(S^{[2]}\right)_{s}=\mathrm{CH}_{\mathrm{CK}}^{p}\left(S^{[2]}\right)_{s} .
$$

Consequently, the Fourier decomposition is compatible with intersection product, i.e, the conclusion of Theorem 3 holds for $F=S^{[2]}$.

Remark 15.9. One can check that the cycle $L$ representing the Beauville-Bogomolov class defined in (92) belongs to the graded piece $\mathrm{CH}_{\mathrm{CK}}^{2}\left(S^{[2]} \times S^{[2]}\right)_{0}$. Here, the Chow-Künneth decomposition of $S^{[2]} \times S^{[2]}$ is understood to be the product Chow-Künneth decomposition of the multiplicative Chow-Künneth decomposition of $S^{[2]}$ given by Theorem 6 .

\section{The CyCle $L$ of $S^{[2]}$ VIA MOduli of Stable Sheaves}

Let $F=S^{[2]}$ for some K3 surface $S$. So far, we have defined two cycles $L \in \mathrm{CH}^{2}(F \times F)$ lifting the Beauville-Bogomolov class $\mathfrak{B}$. The first one was defined in (53) using Markman's twisted sheaf $\mathscr{M}$ of Definition 9.13 on $F \times F$ with $F$ seen as the moduli space of stable sheaves on $S$ with Mukai vector $v=(1,0,-1)$, while the second one was defined in (92) using the incidence correspondence $I$. The goal of this section is to prove that these two cycles agree.

Proposition 16.1. The cycle $L$ defined in (92) agrees with the cycle $L$ defined in (53).

Let $Z \subset F \times S$ be the universal family of length-2 subschemes of $S$. Let $\mathcal{I}$ be the ideal sheaf defining $Z$ as a subscheme of $F \times S$. In this way, we realize $F$ as the moduli space of stable sheaves with Mukai vector $v=(1,0,-1)$ and $\mathcal{I}$ is the universal sheaf. As before, we use $p: Z \rightarrow F$ and $q: Z \rightarrow S$ to denote the natural projections. These are the restriction to $Z$ of the natural projections $\pi_{F}$ and $\pi_{S}$ from $F \times S$ to the corresponding factors.

Lemma 16.2. Let $Y$ be a smooth projective variety and $W \subset Y$ a smooth closed sub-variety. If $\mathcal{I}_{W}$ is the ideal sheaf defining the sub-variety $W$, then

$$
\operatorname{ch}\left(\mathcal{I}_{W}\right)=1-i_{W_{*}}\left(\frac{1}{\operatorname{td}\left(\mathscr{N}_{W / Y}\right)}\right)
$$

where $i_{W}: W \hookrightarrow Y$ is the natural closed embedding.

Proof. There is a short exact sequence

$$
0 \longrightarrow \mathcal{I}_{W} \longrightarrow \mathcal{O}_{Y} \longrightarrow i_{W_{*}} \mathcal{O}_{W} \longrightarrow 0
$$


on $Y$ and hence we have $\operatorname{ch}\left(\mathcal{I}_{W}\right)=1-\operatorname{ch}\left(i_{W_{*}} \mathcal{O}_{W}\right)$. If we apply the Grothendieck-Riemann-Roch theorem to the morphism $i_{W}$ and the structure sheaf $\mathcal{O}_{W}$, we get

$$
\begin{aligned}
\operatorname{ch}\left(i_{W_{*}} \mathcal{O}_{W}\right) & =\frac{i_{W *}\left(\operatorname{ch}\left(\mathcal{O}_{W}\right) \operatorname{td}_{W}\right)}{\operatorname{td}_{Y}} \\
& =\frac{i_{W_{*}} \operatorname{td}_{W}}{\operatorname{td}_{Y}} \\
& =i_{W_{*}}\left(\frac{1}{\operatorname{td}\left(\mathscr{N}_{W / Y}\right)}\right),
\end{aligned}
$$

which proves the lemma.

Proof of Proposition 16.1. Considering the universal family $i_{Z}: Z \hookrightarrow F \times S$, we easily deduce from (64) that

$$
c_{1}\left(\mathscr{N}_{Z / F \times S}\right)=E, \quad c_{2}\left(\mathscr{N}_{Z / F \times S}\right)=24 q^{*} \mathfrak{o}_{S}-E^{2}, \quad c_{3}\left(\mathscr{N}_{Z / F \times S}\right)=0, \quad c_{4}\left(\mathscr{N}_{Z / F \times S}\right)=0 .
$$

Then the Todd classes of $\mathscr{N}_{Z / F \times S}$ can be written explicitly as

$$
\operatorname{td}_{1}\left(\mathscr{N}_{Z / F \times S}\right)=\frac{1}{2} E, \quad \operatorname{td}_{2}\left(\mathscr{N}_{Z / F \times S}\right)=2 q^{*} \mathfrak{o}_{S}, \quad \operatorname{td}_{3}\left(\mathscr{N}_{Z / F \times S}\right)=2 q^{*} \mathfrak{o}_{S} \cdot E, \quad \operatorname{td}_{4}\left(\mathscr{N}_{Z / F \times S}\right)=0,
$$

where we use the fact that $E^{3}=-24 j_{Z *} \eta^{*} \mathfrak{o}_{S}$. By Lemma 16.2, we have

$$
\operatorname{ch}(\mathcal{I})=1-Z+\frac{1}{2} \pi_{F}^{*} \delta \cdot Z+\left(2 \pi_{S}^{*} \mathfrak{o}_{S} \cdot Z-\frac{1}{4} \pi_{F}^{*} \delta^{2} \cdot Z\right)+\frac{1}{8} \pi_{F}^{*} \delta^{3} \cdot Z+\frac{1}{2} \pi_{F}^{*} \mathfrak{o}_{F} \cdot Z .
$$

Let $\mathscr{E}=\mathrm{R} p_{12_{*}}\left(p_{13}^{*} \mathcal{I}^{\vee} \otimes p_{23}^{*} \mathcal{I}\right)$, where $p_{i j}$ and $p_{k}$ are the natural projections from $F \times F \times S$ and all the push-forward, pull-back and tensor product are in the derived sense. Then, by the GrothendieckRiemann-Roch formula, we have

$$
\operatorname{ch}(\mathscr{E})=p_{12_{*}}\left(p_{13}^{*} \operatorname{ch}^{\vee}(\mathcal{I}) \cdot p_{23}^{*} \operatorname{ch}(\mathcal{I}) \cdot p_{3}^{*} \operatorname{td}_{S}\right) .
$$

We put equation (101) into the above formula and eventually get

$$
\operatorname{ch}(\mathscr{E})=-2+\left(-\delta_{1}+\delta_{2}\right)+\left(I-\frac{1}{2} \delta_{1}^{2}-\frac{1}{2} \delta_{2}^{2}\right)+\cdots,
$$

where $I$ is the incidence correspondence of Definition 11.1. Let $\mathscr{E}^{i}:=\mathcal{E} x t_{p_{12}}^{i}\left(p_{13}^{*} \mathcal{I}, p_{23}^{*} \mathcal{I}\right), i=0,1,2$. Note that in the current situation, Markman's sheaf $\mathscr{M}$ is simply $\mathscr{E}^{1}$. In the $K$-group, we have

$$
[\mathscr{E}]=\left[\mathscr{E}^{0}\right]-\left[\mathscr{E}^{1}\right]+\left[\mathscr{E}^{2}\right]
$$

By Lemma $9.8, \mathscr{E}^{0}$ and $\mathscr{E}^{2}$ do not contribute to lower degree terms in the total Chern character. Hence $\operatorname{ch}\left(\mathscr{E}^{1}\right)$ agrees with $-\operatorname{ch}(\mathscr{E})$ in low degree terms. To be more precise, we have

$$
\operatorname{ch}\left(\mathscr{E}^{1}\right)=2+\left(\delta_{1}-\delta_{2}\right)+\left(-I+\frac{1}{2} \delta_{1}^{2}+\frac{1}{2} \delta_{2}^{2}\right)+\cdots .
$$

It follows that

$$
\kappa\left(\mathscr{E}^{1}\right)=\operatorname{ch}\left(\mathscr{E}^{1}\right) \exp \left(-c_{1}\left(\mathscr{E}^{1}\right) / r\right)=2-I+\frac{1}{4} \delta_{1}^{2}+\frac{1}{4} \delta_{2}^{2}+\frac{1}{2} \delta_{1} \delta_{2}+\cdots,
$$

where $r=\operatorname{rk}\left(\mathscr{E}^{1}\right)=2$. Thus we have

$$
\begin{aligned}
\kappa_{2}\left(\mathscr{E}^{1}\right) & =-I+\frac{1}{4} \delta_{1}^{2}+\frac{1}{4} \delta_{2}^{2}+\frac{1}{2} \delta_{1} \delta_{2} \\
& =-L-2 p_{1}^{*} S_{\mathfrak{o}}-2 p_{2}^{*} S_{\mathfrak{o}}++\frac{1}{4} \delta_{1}^{2}+\frac{1}{4} \delta_{2}^{2} \\
& =-L-\frac{1}{12} p_{1}^{*} c_{2}(F)-\frac{1}{12} p_{2}^{*} c_{2}(F),
\end{aligned}
$$

where $p_{i}: F \times F \rightarrow F$ are the projections. Here, $L$ denotes the cycle defined in (92) and the third equality uses Lemma 14.4. One consequence of the above is

$$
\iota_{\Delta}^{*} \kappa_{2}\left(\mathscr{E}^{1}\right)=-l-\frac{1}{6} c_{2}(F)=-c_{2}(F) .
$$

Comparing the above computation with (53) yields the proposition. 


\section{Part 3. The variety of lines on a cubic fourfold}

Fix a 6-dimensional vector space $V$ over $\mathbb{C}$. Let $X \subset \mathbb{P}(V)$ be a smooth cubic fourfold and $F=F(X)$ be the variety of lines on $X$. It is known that $F$ is smooth of dimension 4 ; see [5]. Let $h \in \operatorname{Pic}(X)$ be the class of a hyperplane section on $X$. The variety $F$ naturally embeds into the Grassmannian $G(2, V)$ and hence inherits the tautological rank 2 sub-bundle $\mathscr{E}_{2} \subset V$. Let $g=-c_{1}\left(\mathscr{E}_{2}\right) \in \operatorname{Pic}(F)$ be the Plücker polarization on $F$ and $c=c_{2}\left(\mathscr{E}_{2}\right)$. Set $g_{i}=p_{i}^{*} g \in \mathrm{CH}^{1}(F \times F)$ and $c_{i}=p_{i}^{*} c \in \mathrm{CH}^{2}(F \times F), i=1,2$, where $p_{i}: F \times F \rightarrow F$ are the projections. For all points $x \in X$, let $C_{x}$ be the sub-variety of $F$ parameterizing all lines passing through $x$. If $x \in X$ is general, then $C_{x}$ is a curve (cf. [20, Lemma 2.1]). Voisin [54] showed that $F$ carries a canonical 0 -cycle, $\mathfrak{o}_{F}$, of degree 1 such that the intersection of four divisors is always a multiple of $\mathfrak{o}_{F}$. We always use

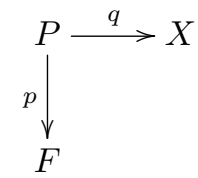

to denote the universal family of lines on $X$.

\section{The INCIDENCE CORRESPONDENCE $I$}

We introduce the incidence correspondence $I \in \mathrm{CH}^{2}(F \times F)$ on the variety of lines on a cubic fourfold. This cycle is the starting point to define the Fourier transform on the Chow ring of $F$. We establish three core identities involving $I$, namely we compute $I^{2}=I \cdot I$, the pull-back of $I$ along the diagonal embedding $\iota_{\Delta}: F \hookrightarrow F \times F$ and $c_{1} \cdot I$.

If $l \subset X$ is a line, then we use $[l] \in F$ to denote the corresponding point on $F$; If $t \in F$ is a closed point, then we use $l_{t} \subset X$ to denote the corresponding line on $X$.

Definition 17.1. The incidence subscheme $I \subset F \times F$ is defined to be

$$
I:=\left\{(s, t) \in F \times F: l_{s} \cap l_{t} \neq \emptyset\right\},
$$

which is endowed with the reduced closed subscheme structure. Its cycle class in $\mathrm{CH}^{2}(F \times F)$, also denoted $I$ by abuse of notations, is called the incidence correspondence. With the projection onto the first factor $F$, we get a morphism $I \rightarrow F$ whose fiber over a point $[l] \in F$ is denoted $S_{l}$.

It is known that $S_{l}$ is always a surface and smooth if $l$ is general ; see [52]. Consider the following diagram

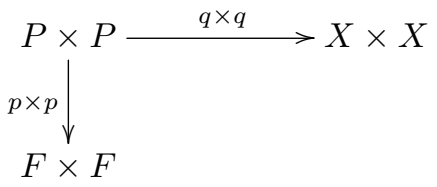

Lemma 17.2. The cycle class of $I$ is given by

$$
I=(p \times p)_{*}(q \times q)^{*} \Delta_{X}, \quad \text { in } \mathrm{CH}^{2}(F \times F) .
$$

Equivalently, if one sees the universal family of lines $P$ on the cubic fourfold $X$ as a correspondence between $F$ and $X$, then

$$
I={ }^{t} P \circ P, \quad \text { in } \mathrm{CH}^{2}(F \times F) .
$$

Proof. For $x \in X$, the fiber $q^{-1}(x)$ is identified with $C_{x}$, the space of lines passing through $x$. In [20, Lemma 2.1], it is shown that $\operatorname{dim} C_{x}=1$ for a general point $x \in X$. By [20, Corollary 2.2], there are at most finitely many points $x_{i} \in X$, called Eckardt points (see [20, Definition 2.3]), such that $C_{x_{i}}$ is a surface. As a consequence, $J:=(q \times q)^{-1} \Delta_{X} \subset P \times P$ is an irreducible variety of dimension 6 . Furthermore, the image of $J$ under the morphism $p \times p$ is equal to $I$. To prove the lemma, it suffices to show that $\left.(p \times p)\right|_{J}: J \rightarrow I$ is of degree 1 . This is clear since a general pair of intersecting lines on $X$ meet transversally in a single point.

For future reference, let us state and prove the following basic general lemma. 
Lemma 17.3. Let $Y$ and $Y^{\prime}$ be smooth projective varieties and $\Gamma \subset Y \times Y^{\prime}$ be a closed sub-variety of codimension $r$. Assume that $\Gamma \rightarrow Y$ is dominant with smooth general fibers. Then

$$
\left(\Gamma^{2}\right)_{*} y=\left(\Gamma_{*} y\right)^{2}
$$

holds in $\mathrm{CH}^{*}(Y)$ for a general point $y \in Y$. Here, $\Gamma^{2}$ denotes the self-intersection $\Gamma \cdot \Gamma \in \mathrm{CH}^{*}\left(Y \times Y^{\prime}\right)$.

Proof. This follows from the following computation.

$$
p_{2, *}\left(\Gamma^{2} \cdot\left(y \times Y^{\prime}\right)\right)=i_{*} c_{r}\left(\left.\mathscr{N}_{\Gamma / Y \times Y^{\prime}}\right|_{\Gamma_{y}}\right)=i_{*} c_{r}\left(\mathscr{N}_{\Gamma_{y} / Y^{\prime}}\right)=\left(\Gamma_{y}\right)^{2},
$$

where $\Gamma_{y}=\Gamma \cap\left(y \times Y^{\prime}\right)$ is viewed as a sub-variety of $Y^{\prime}$ and $i: \Gamma_{y} \hookrightarrow Y^{\prime}$ is the natural embedding.

In [54], C. Voisin established the following identity in $\mathrm{CH}^{4}(F \times F)$,

$$
I^{2}=\alpha \Delta_{F}+I \cdot\left(g_{1}^{2}+g_{1} g_{2}+g_{2}^{2}\right)+\Gamma_{2}\left(g_{1}, g_{2}, c_{1}, c_{2}\right) \text { for some integer } \alpha \neq 0,
$$

where $\Gamma_{2}\left(g_{1}, g_{2}, c_{1}, c_{2}\right)$ is a weighted homogeneous polynomial of degree 4 . Voisin's method actually gives $\Gamma_{2}=6 \Gamma_{h^{2}}-3\left(g_{1}+g_{2}\right) \Gamma_{h}$; see equations (119) and (120) for the notations. Given Proposition A.6, we readily obtain

$$
\Gamma_{2}=-g_{1}^{4}-g_{2}^{4}-g_{1}^{2} c_{2}-g_{2}^{2} c_{1}+2 g_{1}^{2} c_{1}+2 g_{2}^{2} c_{2}-g_{1} g_{2}\left(c_{1}+c_{2}\right)+2 c_{1} c_{2} .
$$

This shows that $\left(\Gamma_{2}\right)_{*}[l]=-g^{4}+2 g^{2} c$, which is of degree -18 . One also sees that

$$
\left(I \cdot\left(g_{1}^{2}+g_{1} g_{2}+g_{2}^{2}\right)\right)_{*}[l]=g^{2} \cdot\left(I_{*}[l]\right),
$$

which is of degree 21 since the cohomology class of $I_{*}[l]$ is equal to that of $\frac{1}{3}\left(g^{2}-c\right)$. Meanwhile, by Lemma 17.3 we have $\left(I^{2}\right)_{*}[l]=\left(I_{*}[l]\right)^{2}$, which is of degree 5 . Letting both sides of the identity (106) act on a point $[l]$ and then counting the degrees, we see that $\alpha=2$. We have thus established the following refinement of Voisin's identity (105).

Proposition 17.4. The following identity holds true in $\mathrm{CH}^{4}(F \times F)$,

$$
I^{2}=2 \Delta_{F}+I \cdot\left(g_{1}^{2}+g_{1} g_{2}+g_{2}^{2}\right)+\Gamma_{2}\left(g_{1}, g_{2}, c_{1}, c_{2}\right),
$$

where $\Gamma_{2}\left(g_{1}, g_{2}, c_{1}, c_{2}\right)$ is a weighted homogeneous polynomial of degree 4 .

The pull-back of $I$ along the diagonal embedding can be expressed in terms of $c$ and $g^{2}$ :

Proposition 17.5. The equation $\iota_{\Delta}^{*} I=6 c-3 g^{2}$ holds in $\mathrm{CH}^{2}(F)$, where $\iota_{\Delta}: F \rightarrow F \times F$ is the diagonal embedding.

Proof. Consider the fiber product square

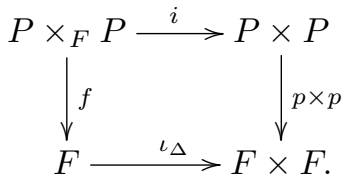

By Lemma 17.2 we have $I=(p \times p)_{*}(q \times q)^{*} \Delta_{X}$ and hence we get

$$
\iota_{\Delta}^{*} I=\iota_{\Delta}^{*}(p \times p)_{*}(q \times q)^{*} \Delta_{X}=f_{*} i^{*}(q \times q)^{*} \Delta_{X} .
$$

Let $\Delta_{P / F}: P \rightarrow P \times_{F} P$ be the diagonal morphism. Then one sees that $i^{*}(q \times q)^{*} \Delta_{X}=\left(\Delta_{P / F}\right)_{*} P$. Consider the following square

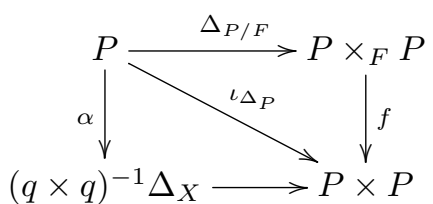

where $\iota_{\Delta_{P}}: P \rightarrow P \times P$ is the diagonal embedding. Let $\mathscr{N}_{1}$ be the normal bundle of $(q \times q)^{-1} \Delta_{X}$ in $P \times P$. Then $\alpha^{*} \mathscr{N}_{1} \cong q^{*} \mathscr{T}_{X}$. Let $\mathscr{N}_{2}$ be the normal bundle of the diagonal $\Delta_{P / F}$ in $P \times_{F} P$. Then $\mathscr{N}_{2}$ is naturally isomorphic to $\mathscr{T}_{P / F}$. The quotient $\alpha^{*} \mathscr{N}_{1} / \mathscr{N}_{2}$ is simply the total normal bundle $\mathscr{N}_{P / X}$. The 
refined intersection formula of Chapter 6 of Fulton [25] shows that $i^{*}(q \times q)^{*} \Delta_{X}=\left(\Delta_{P / F}\right)_{*} c_{3}\left(\mathscr{N}_{P / X}\right)$. By applying $f_{*}$ we get

$$
\iota_{\Delta}^{*} I=f_{*}\left(\Delta_{P / F}\right)_{*} c_{3}\left(\mathscr{N}_{P / X}\right)=p_{*} c_{3}\left(\mathscr{N}_{P / X}\right) .
$$

A routine Chern class computation gives

$$
\begin{aligned}
c\left(\mathscr{N}_{P / X}\right) & =\frac{c\left(q^{*} \mathscr{T}_{X}\right)}{c\left(\mathscr{T}_{P / F}\right)}=\frac{q^{*} c\left(\mathscr{T}_{\mathbb{P}^{5}}\right)}{q^{*} c\left(\mathcal{O}_{X}(3)\right) \cdot c\left(\mathscr{T}_{P / F}\right)} \\
& =\frac{q^{*}\left(1+6 h+15 h^{2}+20 h^{3}+15 h^{4}\right)}{\left(1+3 q^{*} h\right)\left(1+2 q^{*} h-p^{*} g\right)} .
\end{aligned}
$$

It follows that $c_{3}\left(\mathscr{N}_{P / X}\right)=-6 q^{*} h^{3}+6 p^{*} g \cdot q^{*} h^{2}-3 p^{*} g^{2} \cdot q^{*} h+p^{*} g^{3}$. Hence we have $p_{*} c_{3}\left(\mathscr{N}_{P / X}\right)=$ $-6\left(g^{2}-c\right)+6 g^{2}-3 g^{2}=6 c-3 g^{2}$. This concludes the proof.

The following lifting lemma is essential to getting statements that do not depend on the choice of a polarization. Specifically, Lemma 17.6 is to the variety of lines on a cubic fourfold what Lemma 14.3 is to the Hilbert scheme of length-2 subschemes on a K3 surface : it is essential to establishing Conjecture 1 for $F$.

Lemma 17.6. The following relation holds in $\mathrm{CH}^{4}(F \times F)$ :

$$
c_{1} \cdot I=P\left(g_{1}, g_{2}, c_{1}, c_{2}\right)
$$

for some weighted homogeneous polynomial $P$ of degree 4 .

Proof. Let $H_{1} \subset X$ be a general hyperplane section of $X$. Then the class $c$ can be represented by the surface of all lines contained in $H_{1}$. Then $c_{1} \cdot I$ is represented by the following cycle

$$
Z_{1}:=\left\{\left(\left[l_{1}\right],\left[l_{2}\right]\right) \in F \times F: l_{1} \subset H_{1}, l_{1} \cap l_{2} \neq \emptyset\right\} .
$$

Let $H_{2} \subset X$ be another general hyperplane section of $X$ and let

$$
Y_{1}:=\left\{[l] \in F: l \cap H_{1} \cap H_{2} \neq \emptyset\right\} .
$$

Note that the cycle class of $Y_{1}$ is simply $g$. The following two cycles are defined in Appendix A, equations (119) and (120) respectively.

$$
\begin{gathered}
\Gamma_{h}=\left\{\left(\left[l_{1}\right],\left[l_{2}\right]\right) \in F \times F: \exists x \in H_{1}, x \in l_{1} \cap l_{2}\right\} ; \\
\Gamma_{h^{2}}=\left\{\left(\left[l_{1}\right],\left[l_{2}\right]\right) \in F \times F: \exists x \in H_{1} \cap H_{2}, x \in l_{1} \cap l_{2}\right\} .
\end{gathered}
$$

We have the following scheme-theoretic intersection

$$
\left(Y_{1} \times F\right) \cap \Gamma_{h}=Z_{1} \cup \Gamma_{h^{2}}
$$

It follows that $g_{1} \cdot \Gamma_{h}$ is a linear combination of $Z_{1}$ and $\Gamma_{h^{2}}$. Or equivalently $c_{1} \cdot I$ can be written as a linear combination of $g_{1} \cdot \Gamma_{h}$ and $\Gamma_{h^{2}}$. It follows from Proposition A.6 that $c_{1} \cdot I$ is a polynomial in $\left(g_{1}, g_{2}, c_{1}, c_{2}\right)$.

\section{The Rational SELF-MaP $\varphi: F \rightarrow F$}

The goal of this section is to understand the action of a rational map $\varphi: F \rightarrow F$, first constructed by Voisin [53], on zero-cycles on $F$. We state and prove two lemmas that will be used in Section 19 to show Theorem 2 for $F$.

We refer to Appendix A for definitions and notations. In [53], Voisin defines a rational self-map $\varphi: F \rightarrow F$ as follows. If a line $l \subset X$ is of first type, then there is a unique plane $\Pi_{l}$ which contains $l$ and is tangent to $X$ along $l$. If $\Pi_{l}$ is not contained in $X$, then we have

$$
\Pi_{l} \cdot X=2 l+l^{\prime}
$$

for some line $l^{\prime}$. Let $\Sigma_{1} \subset F$ be the sub-variety of lines contained in some linear plane $\mathbb{P}^{2} \subset X$. When $X$ does not contain any plane, then $\Sigma_{1}=\emptyset$. If $X$ contains at least a plane, then $\Sigma_{1}$ is a disjoint union of $\mathbb{P}^{2}$ 's. Let $\Sigma_{2} \subset F$ be the surface of lines of second type.

Definition 18.1 ([53]). Let $\varphi: F \backslash\left(\Sigma_{1} \cup \Sigma_{2}\right) \rightarrow F$ be the morphism defined by $\varphi([l])=\left[l^{\prime}\right]$. 
Lemma 18.2 below will be used in the proof of Proposition 19.4 in order to establish conditions (7) and (8) in the case where $F$ is the variety of lines on a cubic fourfold. Note that Lemma 18.2 can be seen as a consequence of Proposition 21.3. We have however decided for clarity to postpone the more detailed study of $\varphi$ until after the Fourier decomposition for $F$ is established.

Lemma 18.2. For any point $[l] \in F$, we have

$$
g^{2} \cdot I_{*}[l]=\varphi_{*}[l]-4[l]+24 \mathfrak{o}_{F} \in \mathrm{CH}_{0}(F) .
$$

Proof. We may assume that the point $[l]$ is general. We first recall some results on the geometry of the surface $S_{l} \subset F$ of all lines meeting $l \subset X$. Our main reference for this is [52]. It is known that for general $l$ the surface $S_{l}$ is smooth. On $S_{l}$ we have an involution $\iota$ which is defined as follows. Let $\left[l_{1}\right] \in S_{l}$ which is not $[l]$ then $\iota\left(\left[l_{1}\right]\right)$ is the residue line of $l \cup l_{1}$. If $l_{1}=l$, then $\iota([l])=\varphi([l])$. There are two natural divisors on $S_{l}$. The first one is $C_{x}$ which is the curve parameterizing all the lines passing through a point $x \in l$. The second one is $C_{x}^{\iota}=\iota\left(C_{x}\right)$. Then we have

$$
C_{x}^{2}=[l], \quad\left(C_{x}^{\iota}\right)^{2}=\iota([l]),\left.\quad g\right|_{S_{l}}=2 C_{x}+C_{x}^{\iota} .
$$

Let $j: S_{l} \rightarrow F$ be the natural inclusion, then

$$
g^{2} \cdot I_{*}[l]=g^{2} \cdot S_{l}=j_{*}\left(j^{*} g^{2}\right)=j_{*}\left(2 C_{x}+C_{x}^{\iota}\right)^{2}=j_{*}\left(4[l]+4 C_{x} \cdot C_{x}^{\iota}+\iota([l])\right)
$$

One notes that $j_{*}\left(2[l]+C_{x} C_{x}^{\iota}\right)=j_{*}\left(C_{x}\right) \cdot g=6 \mathfrak{o}_{F}$. The lemma follows easily.

We now study the action of $\varphi$ on points $s \in \Sigma_{2}$, that is, on points $s \in F$ whose corresponding line $l=l_{s}$ is of second type ; see Appendix A. The following lemma is crucial to establishing condition (9) for $F$; see Proposition 19.6.

Lemma 18.3. Let $s \in \Sigma_{2}$, then we have

$$
\varphi_{*} s=-2 s+3 \mathfrak{o}_{F} \in \mathrm{CH}_{0}(F) .
$$

Proof. We write $l=l_{s} \subset X$. Let $\mathbb{P}_{\langle l\rangle}^{3}$ be the linear $\mathbb{P}^{3}$ tangent to $X$ along $l$. Let $S=\mathbb{P}_{\langle l\rangle}^{3} \cap X$. Then there is a canonical curve $l^{\prime} \subset S$ such that for any point $s^{\prime} \in \mathcal{E}_{[l]}$ the corresponding line $l_{s^{\prime}}$ meets both $l$ and $l^{\prime}$. Let $x \in l$ be a general point, then there are two points $s_{1}^{\prime}, s_{2}^{\prime} \in \mathcal{E}_{[l]}$ with corresponding lines $l_{1}$ and $l_{2}$ such that both of them pass through the point $x \in l$; see Proposition A.11. In Lemma A.8, we established the following fact : if $L_{1}$ and $L_{2}$ are two intersecting lines and $L_{t}$ a family of lines that specialize to the line $L_{2}$, then four secant lines of $\left(L_{1}, L_{t}\right)$ specialize to four lines $E_{1}, \ldots, E_{4}$ passing through $x=L_{1} \cap L_{2}$ with

$$
\left[L_{1}\right]+\left[L_{2}\right]+\left[E_{1}\right]+\left[E_{2}\right]+\left[E_{3}\right]+\left[E_{4}\right]=6 \mathfrak{o}_{F}, \quad \text { in } \mathrm{CH}_{0}(F)
$$

and the fifth secant line specializes to the residue line of $L_{1}$ and $L_{2}$. Now we apply this specialization argument by taking $L_{1}=l_{1}, L_{2}=l_{2}$ and $L_{t}=l_{t}, t \in \mathcal{E}_{[l]} \backslash\left\{s_{1}, s_{2}\right\}$ with $t \rightarrow s_{2}$. By Proposition A.12, the secant lines of $\left(l_{1}, l_{t}\right)$ are constantly given by $4 l+l^{\prime}$. In particular the limit is again $4 l+l^{\prime}$. Note that $l^{\prime}$ is the residue line of $l_{1}$ and $l_{2}$. Hence we have

$$
4[l]+\left[l_{1}\right]+\left[l_{2}\right]=6 \mathfrak{o}_{F} .
$$

The following claim shows that $\left[l_{1}\right]=\left[l_{2}\right]=\varphi_{*}[l]$ in $\mathrm{CH}_{0}(F)$.

Claim. Let $s=[l] \in \Sigma_{2}$, then $\varphi_{*} s=s^{\prime}$ in $\mathrm{CH}_{0}(F)$, where $s^{\prime} \in \mathcal{E}_{[l]}$ is an arbitrary point.

Proof of Claim. Consider a general curve $C \subset F$ such that $C$ meets the indeterminacy loci of $\varphi$ in the single point $s \in \Sigma_{2}$. Then $\left.\varphi\right|_{C \backslash\{s\}}$ extends to a morphism $\varphi^{\prime}: C \rightarrow F$ such that $s^{\prime}=\varphi^{\prime}(s) \in \mathcal{E}_{[l]}$. Note that $s$ is rationally equivalent to a cycle $\gamma$ supported on $C \backslash\{s\}$. Then by definition, we have

$$
\varphi_{*} s=\varphi_{*} \gamma=\varphi_{*}^{\prime} \gamma=\varphi_{*}^{\prime} s=s^{\prime} .
$$

Since $\mathcal{E}_{[l]}$ is a rational curve, any point $s^{\prime} \in \mathcal{E}_{[l]}$ represents $\varphi_{*} s$.

The lemma is now proved. 


\section{The Fourier DeCOMPosition For $F$}

In this section, we prove Theorem 2 for the variety of lines $F$ on a cubic fourfold. First we construct a cycle $L$ in $\mathrm{CH}^{2}(F \times F)$ that represents the Beauville-Bogomolov form $\mathfrak{B}$, and we show (Theorem 19.2) that $L$ satisfies Conjecture 1 , i.e., that $L^{2}=2 \Delta_{F}-\frac{2}{25}\left(l_{1}+l_{2}\right) \cdot L-\frac{1}{23 \cdot 25}\left(2 l_{1}^{2}-23 l_{1} l_{2}+2 l_{2}^{2}\right)$ in $\mathrm{CH}^{4}(F \times F)$. Then we check that Properties (7) and (8) are satisfied by $L$ (Proposition 19.4). We can thus apply Theorem 2.2. Finally, we show that $L$ satisfies Property (9) (Proposition 19.6) so that we can apply Theorem 2.4 and get the Fourier decomposition of Theorem 2 for $F$.

Let $\left\{\mathfrak{a}_{1}, \mathfrak{a}_{2}, \ldots, \mathfrak{a}_{23}\right\}$ be a basis of $H^{4}(X, \mathbb{Z})$. We use $b_{0}: H^{4}(X, \mathbb{Z}) \times H^{4}(X, \mathbb{Z}) \rightarrow \mathbb{Z},\left(\mathfrak{a}, \mathfrak{a}^{\prime}\right) \mapsto \int_{X} \mathfrak{a} \cup \mathfrak{a}^{\prime}$ to denote the intersection pairing on $\mathrm{H}^{4}(X, \mathbb{Z})$. Let $A=\left(b_{0}\left(\mathfrak{a}_{i}, \mathfrak{a}_{j}\right)\right)_{1 \leq i, j \leq 23}$ be the intersection matrix. For any $\mathfrak{a} \in \mathrm{H}^{4}(X, \mathbb{Q})$ we define $\hat{\mathfrak{a}}=p_{*} q^{*} \mathfrak{a} \in \mathrm{H}^{2}(F, \mathbb{Q})$. Then by a result of Beauville and Donagi [10], the set $\left\{\hat{\mathfrak{a}}_{1}, \ldots, \hat{\mathfrak{a}}_{23}\right\}$ forms a basis of $\mathrm{H}^{2}(F, \mathbb{Z})$. Consider now $\Lambda:=\mathrm{H}^{2}(F, \mathbb{Z})$ endowed with the Beauville-Bogomolov bilinear form $q_{F}$. Then $q_{F}$ can be described as follows

$$
q_{F}\left(\hat{\mathfrak{a}}, \hat{\mathfrak{a}^{\prime}}\right)=b_{0}\left(\mathfrak{a}, h^{2}\right) b_{0}\left(\mathfrak{a}^{\prime}, h^{2}\right)-b_{0}\left(\mathfrak{a}, \mathfrak{a}^{\prime}\right) .
$$

Hence as in equation (10) we obtain a well-defined element

$$
q_{F}^{-1} \in \operatorname{Sym}^{2}(\Lambda)\left[\frac{1}{2}\right] \subset \Lambda \otimes \Lambda\left[\frac{1}{2}\right] \subset \mathrm{H}^{4}(F \times F, \mathbb{Q}) .
$$

Proposition 19.1. The cohomological class $[I] \in \mathrm{H}^{4}(F \times F, \mathbb{Z})$ is given by

$$
[I]=\frac{1}{3}\left(g_{1}^{2}+\frac{3}{2} g_{1} g_{2}+g_{2}^{2}-c_{1}-c_{2}\right)-q_{F}^{-1} .
$$

Proof. Consider the diagram (104). By Lemma 17.2 we have $I=(p \times p)_{*}(q \times q)^{*} \Delta_{X}$. The cohomology class of the diagonal $\Delta_{X} \subset X \times X$ is given by

$$
\left[\Delta_{X}\right]=p t \times[X]+[X] \times p t+\frac{1}{3}\left(h \otimes h^{3}+h^{3} \otimes h\right)+b_{0}^{-1},
$$

where $b_{0}: \mathrm{H}^{4}(X, \mathbb{Z}) \times \mathrm{H}^{4}(X, \mathbb{Z}) \rightarrow \mathbb{Z},\left(\mathfrak{a}, \mathfrak{a}^{\prime}\right) \mapsto \int_{X} \mathfrak{a} \cup \mathfrak{a}^{\prime}$ is the intersection pairing. Then the cohomology class of $I$ can be computed as

$$
\begin{aligned}
{[I] } & =(p \times p)_{*}(q \times q)^{*}\left[\Delta_{X}\right] \\
& =\frac{1}{3}\left(p_{1}^{*}\left(p_{*} q^{*} h^{3}\right)+p_{2}^{*}\left(p_{*} q^{*} h^{3}\right)\right)-(p \times p)_{*}(q \times q)^{*} b_{0}^{-1} \\
& =\frac{1}{3}\left(g_{1}^{2}+g_{2}^{2}-c_{1}-c_{2}\right)-(p \times p)_{*}(q \times q)^{*} b_{0}^{-1}
\end{aligned}
$$

Here, we used the fact that $p_{*} q^{*} h^{3}=g^{2}-c$; see Lemma A.4. We take a basis $\left\{\mathfrak{a}_{1}^{\prime}=h^{2}, \mathfrak{a}_{2}^{\prime}, \ldots, \mathfrak{a}_{23}^{\prime}\right\}$ of $\mathrm{H}^{4}(X, \mathbb{Q})$ such that $b_{0}\left(h^{2}, \mathfrak{a}_{i}^{\prime}\right):=\int_{X} h^{2} \cup \mathfrak{a}_{i}^{\prime}=0$, for all $2 \leq i \leq 23$. Let $A^{\prime}=\left(b_{0}\left(\mathfrak{a}_{i}^{\prime}, \mathfrak{a}_{j}^{\prime}\right)\right)_{2 \leq i, j \leq 23}$ and $B^{\prime}=A^{\prime-1}$. Then we have

$$
b_{0}^{-1}=\frac{1}{3} h^{2} \otimes h^{2}+\sum_{2 \leq i, j \leq 23} b_{i j}^{\prime} \mathfrak{a}_{i}^{\prime} \otimes \mathfrak{a}_{j}^{\prime} .
$$

On $F$, the Beauville-Bogomolov bilinear form $q_{F}$ is given by $q_{F}(g, g)=6$ and $q_{F}\left(\hat{\mathfrak{a}}_{i}^{\prime}, \hat{\mathfrak{a}}_{j}^{\prime}\right)=-a_{i j}^{\prime}$, for all $2 \leq i, j \leq 23$. It follows that

Hence we have

$$
q_{F}^{-1}=\frac{1}{6} g \otimes g-\sum_{2 \leq i, j \leq 23} b_{i j}^{\prime} \hat{\mathfrak{a}}_{i}^{\prime} \otimes \hat{\mathfrak{a}}_{j}^{\prime} .
$$

$$
(p \times p)_{*}(q \times q)^{*} b_{0}^{-1}=\frac{1}{3} g_{1} g_{2}+\sum_{2 \leq i, j \leq 23} b_{i j}^{\prime} p_{1}^{*} \hat{\mathfrak{a}}_{i}^{\prime} p_{2}^{*} \hat{\mathfrak{a}}_{j}^{\prime}=\frac{1}{2} g_{1} g_{2}-q_{F}^{-1} .
$$

Combining this with the expression of $[I]$ obtained above gives

$$
[I]=\frac{1}{3}\left(g_{1}^{2}+\frac{3}{2} g_{1} g_{2}+g_{2}^{2}-c_{1}-c_{2}\right)-q_{F}^{-1}
$$

which completes the proof. 
Thus it follows from Proposition 19.1 and its proof that the cycle

$$
L:=\frac{1}{3}\left(g_{1}^{2}+\frac{3}{2} g_{1} g_{2}+g_{2}^{2}-c_{1}-c_{2}\right)-I
$$

represents the Beauville-Bogomolov class $\mathfrak{B}$. Also, we see that the cohomology class of the cycle

$$
L_{g}:=\frac{1}{3}\left(g_{1}^{2}+g_{1} g_{2}+g_{2}^{2}-c_{1}-c_{2}\right)-I
$$

is $\left(\left.q_{F}\right|_{\mathrm{H}^{2}(F, \mathbb{Z})_{\text {prim }}}\right)^{-1}$, where $\mathrm{H}^{2}(F, \mathbb{Z})_{\text {prim }}=g^{\perp}=p_{*} q^{*} \mathrm{H}^{4}(X, \mathbb{Z})_{\text {prim }}$.

Note that Proposition 17.5 gives

$$
l:=\left(\iota_{\Delta}\right)^{*} L=\frac{7}{6} g^{2}-\frac{2}{3} c-\iota_{\Delta}^{*} I=\frac{25}{6} g^{2}-\frac{20}{3} c=\frac{5}{6} c_{2}(F) .
$$

The last step follows from Lemma A.1. One should compare this with equation (93).

Theorem 19.2. The cycle $L$ of (107) satisfies Conjecture 1.

Proof. Using Voisin's identity (106) and the definition (107) of $L$, we easily get

$$
L^{2}=2 \Delta_{F}-\frac{1}{3}\left(g_{1}^{2}+g_{2}^{2}+2 c_{1}+2 c_{2}\right) \cdot L+\Gamma_{4},
$$

where $\Gamma_{4}$ is a weighted homogeneous polynomial of degree 4 in $\left(g_{1}, g_{2}, c_{1}, c_{3}\right)$. Note that Lemma 17.6 implies that $c_{i} \cdot L$ is a weighted homogeneous polynomial in $\left(g_{1}, g_{2}, c_{1}, c_{2}\right)$. Hence we can modify the term in the middle by $c_{i} \cdot L$ and get

$$
L^{2}=2 \Delta_{F}-\frac{1}{3}\left(g_{1}^{2}+g_{2}^{2}-\frac{8}{5} c_{1}-\frac{8}{5} c_{2}\right) \cdot L+\Gamma_{5},
$$

where $\Gamma_{5}$ is a degree 4 polynomial in $\left(g_{1}, g_{2}, c_{1}, c_{2}\right)$. Equation (108) gives

$$
\frac{1}{3}\left(g_{1}^{2}+g_{2}^{2}-\frac{8}{5} c_{1}-\frac{8}{5} c_{2}\right)=\frac{2}{25}\left(l_{1}+l_{2}\right)
$$

where $l_{i}=p_{i}^{*} l$ as before. Thus we have the following equation

$$
L^{2}=2 \Delta_{F}-\frac{2}{25}\left(l_{1}+l_{2}\right) \cdot L+\Gamma_{5}
$$

Comparing this with equation (12), we see that $\Gamma_{5}$ is cohomologically equivalent to $\frac{1}{23 \cdot 25}\left(2 l_{1}^{2}+23 l_{1} l_{2}+2 l_{2}^{2}\right)$. Lemma 4.8 yields $\Gamma_{5}=\frac{1}{23 \cdot 25}\left(2 l_{1}^{2}+23 l_{1} l_{2}+2 l_{2}^{2}\right)$.

Lemma 19.3. The cycle $L$ acts as zero on $\mathfrak{o}_{F}$ and on triangles $([l],[l], \varphi([l]))$. Precisely,

(i) $L_{*} \mathfrak{o}_{F}=0$;

(ii) $L_{*}\left(\varphi_{*}+2\right)=0$ on $\mathrm{CH}_{0}(F)$, where $\varphi$ is as in Definition 18.1 .

Proof. Using the definition of $L$, we have

$$
L_{*} \mathfrak{o}_{F}=\frac{1}{3}\left(g^{2}-c\right)-I_{*} \mathfrak{o}_{F}=0,
$$

where the last equality uses Lemma A.5. This proves statement (i). Let $t \in F$, then we have

$$
I_{*}\left(\varphi_{*} t+2 t\right)=\Phi\left(\Psi\left(\varphi_{*} t+2 t\right)\right)=\Phi\left(h^{3}\right)=g^{2}-c
$$

where the last equality uses Lemma A.4. By the definition of $L$, we get

$$
L_{*}\left(\varphi_{*} t+2 t\right)=\left(g^{2}-c\right)-I_{*}\left(\varphi_{*} t+2 t\right)=0 .
$$

This proves statement (ii).

We now check that the cycle $L$ satisfies conditions (7) and (8) of Theorem 2.2 :

Proposition 19.4. We have $L_{*} l^{2}=0$ and $L_{*}\left(l \cdot L_{*} \sigma\right)=25 L_{*} \sigma$ for all $\sigma \in \mathrm{CH}_{0}(F)$. 
Proof. By equation (108), we see that $l^{2}$ is proportional to $c_{2}(F)^{2}$. By [54], $l^{2}$ is a multiple of $\mathfrak{o}_{F}$. Hence we only need to prove that $L_{*} \mathfrak{o}_{F}=0$, which is statement (i) of Lemma 19.3. To prove the second equality, we take an arbitrary closed point $t \in F$. Then we have

$$
\begin{aligned}
l \cdot L_{*} t & =\left(\frac{25}{6} g^{2}-\frac{20}{3} c\right) \cdot\left(\frac{1}{3}\left(g^{2}-c\right)-I_{*} t\right) \\
& =a \mathfrak{o}_{F}-\frac{25}{6} g^{2} \cdot I_{*} t, \quad a \in \mathbb{Z} \\
& =a \mathfrak{o}_{F}-\frac{25}{6}\left(\varphi_{*} t-4 t+24 \mathfrak{o}_{F}\right) \\
& =a^{\prime} \mathfrak{o}_{F}-\frac{25}{6}\left(\varphi_{*} t+2 t\right)+25 t, \quad a^{\prime}=a-100 .
\end{aligned}
$$

Here the first equality uses the definition of $L$ and the explicit expression of $l$ as given in equation (108). The second equality used the fact that any degree 4 polynomial in $(g, c)$ is a multiple of $\mathfrak{o}_{F}$; see [54]. The third equality uses Lemma 18.2. We apply $L_{*}$ to the above equality and use Lemma 19.3, we get

$$
L_{*}\left(l \cdot L_{*} t\right)=0+0+25 L_{*} t=25 L_{*} t .
$$

This establishes the second equality.

We now check that the cycle $L$ satisfies condition (9) of Theorem 2.4. By Theorem 19.2 and Proposition 19.4, we see that $\mathrm{CH}^{4}(F)\left(=\mathrm{CH}_{0}(F)\right)$ has a Fourier decomposition as in Theorem 2.4. First, we have the following.

Proposition 19.5. We have

$$
\mathrm{CH}^{4}(F)_{0} \oplus \mathrm{CH}^{4}(F)_{2}=\operatorname{im}\left\{\mathrm{CH}_{0}\left(\Sigma_{2}\right) \rightarrow \mathrm{CH}_{0}(F)\right\} .
$$

Proof. Recall that (108) $l=\frac{25}{6} g^{2}-\frac{20}{3} c$, that $c \cdot \sigma$ is a multiple of $\mathfrak{o}_{F}$ for all $\sigma \in \mathrm{CH}^{2}(F)$ (Lemma A.3) and that $\Sigma_{2}=5\left(g^{2}-c\right)$ (Lemma A.9). Therefore $l \cdot \sigma$ is proportional to $\Sigma_{2} \cdot \sigma$ for all $\sigma \in \mathrm{CH}^{2}(F)$. Now that Theorem 19.2 and Proposition 19.4 have been proved, we know from Theorems $2.2 \& 2.4$ that $\mathrm{CH}^{4}(F)_{0}=\left\langle l^{2}\right\rangle$ and that $\mathrm{CH}^{4}(F)_{2}=l \cdot \mathrm{CH}^{2}(F)_{2}$. Hence $\mathrm{CH}^{4}(F)_{0} \oplus \mathrm{CH}^{4}(F)_{2} \subseteq \operatorname{im}\left\{\Sigma_{2} \cdot: \mathrm{CH}^{2}(F) \rightarrow\right.$ $\left.\mathrm{CH}^{4}(F)\right\}$. Consider now a zero-cycle $\tau \in \mathrm{CH}^{4}(F)$ which is supported on $\Sigma_{2}$. The key point is then Lemma 18.3 which gives $\varphi_{*} \tau=-2 \tau+3 \operatorname{deg}(\tau) \mathfrak{o}_{F} \in \mathrm{CH}^{4}(F)$. We also have, by Lemma $18.2, g^{2} \cdot I_{*}(\tau)=$ $\varphi_{*} \tau-4 \tau+24 \operatorname{deg}(\tau) \mathfrak{o}_{F}$. It follows that $\tau$ is a linear combination of $l \cdot L_{*}(\tau)$ and $\mathfrak{o}_{F}$. By Theorems 2.2 $\& 2.4, l \cdot L_{*}(\tau)$ and $\mathfrak{o}_{F}$ belong respectively to $\mathrm{CH}^{4}(F)_{2}$ and $\mathrm{CH}^{4}(F)_{0}$ and we are done.

Proposition 19.6. $\left(L^{2}\right)_{*}\left(l \cdot\left(L^{2}\right)_{*} \sigma\right)=0$ for all $\sigma \in \mathrm{CH}^{2}(F)$.

Proof. By Theorem 19.2 and Proposition 19.4, we see that the decomposition in Theorem 2.2 holds on $F$. Thus we have eigenspace decompositions $\mathrm{CH}^{4}(F)=\Lambda_{0}^{4} \oplus \Lambda_{2}^{4}$ and $\mathrm{CH}^{2}(F)=\Lambda_{25}^{2} \oplus \Lambda_{2}^{2} \oplus \Lambda_{0}^{2}$ for the action of $L^{2}$. Moreover, $\Lambda_{25}^{2}=\langle l\rangle$ and Lemma 2.3 gives $\left(L^{2}\right)_{*} l^{2}=0$. Therefore, it is sufficient to prove that $l \cdot \sigma=0$ for all $\sigma \in \Lambda_{2}^{2}$. According to (37), $\sigma \in \Lambda_{2}^{2}$ if and only if $l \cdot \sigma \in \Lambda_{2}^{4}$. In order to conclude it is enough to show that $l \cdot \sigma \in \Lambda_{0}^{4}$, where $\Lambda_{0}^{4}=\mathrm{CH}^{4}(F)_{0} \oplus \mathrm{CH}^{4}(F)_{2}$ by Theorem 2.4. But as in the proof of Proposition 19.5, we see that $l \cdot \sigma$ is proportional to $\Sigma_{2} \cdot \sigma$ so that the statement of Proposition 19.5 yields the result.

By Theorems 2.2 and 2.4, the Fourier decomposition as in Theorem 2 is now established for the variety of lines on a cubic fourfold.

\section{A First multiplicative RESUlt}

Let us start with the following definition.

Definition 20.1. We set

$$
\begin{aligned}
\mathrm{F}^{2} \mathrm{CH}_{\mathbb{Z}}^{4}(F) & =\mathrm{F}^{1} \mathrm{CH}_{\mathbb{Z}}^{4}(F)=\operatorname{ker}\left\{c l: \mathrm{CH}_{\mathbb{Z}}^{4}(F) \rightarrow \mathrm{H}^{8}(F, \mathbb{Z})\right\}, \\
\mathrm{F}^{4} \mathrm{CH}_{\mathbb{Z}}^{4}(F) & =\mathrm{F}^{3} \mathrm{CH}_{\mathbb{Z}}^{4}(F)=\operatorname{ker}\left\{I_{*}: \mathrm{CH}_{\mathbb{Z}}^{4}(F) \rightarrow \mathrm{CH}_{\mathbb{Z}}^{2}(F)\right\}, \\
\mathcal{A} & =I_{*} \mathrm{CH}_{\mathbb{Z}}^{4}(F) \subset \mathrm{CH}_{\mathbb{Z}}^{2}(F) .
\end{aligned}
$$


After tensoring with $\mathbb{Q}$, this filtration coincides with the one given in (4) in the introduction ; see the proof of Proposition 20.3.

The main result of this section is the following theorem which shows that the multiplicative structure on the cohomology of $F$ reflects on its Chow groups.

Theorem 20.2. The intersection of 2-cycles on $F$ enjoys the following properties.

(i) The natural homomorphism

$$
\mathrm{CH}_{\mathbb{Z}}^{2}(F) \otimes \mathrm{CH}_{\mathbb{Z}}^{2}(F) \rightarrow \mathrm{CH}_{\mathbb{Z}}^{4}(F)
$$

is surjective ;

(ii) The natural homomorphism

$$
\mathrm{CH}_{\mathbb{Z}}^{2}(F) \otimes \mathrm{CH}_{\mathbb{Z}}^{2}(F)_{\text {hom }} \rightarrow \mathrm{CH}_{\mathbb{Z}}^{4}(F)_{\text {hom }}
$$

is also surjective;

(iii) The image of the natural homomorphism

$$
\mathcal{A}_{\text {hom }} \otimes \mathcal{A}_{\text {hom }} \rightarrow \mathrm{CH}_{\mathbb{Z}}^{4}(F)_{\text {hom }}
$$

is equal to $\mathrm{F}^{4} \mathrm{CH}_{\mathbb{Z}}^{4}(F)$.

Here, the subscript "hom" denotes those cycles that are homologically trivial.

As an immediate corollary of Theorem 20.2(iii), we obtain the following.

Proposition 20.3. We have

$$
\mathrm{CH}^{4}(F)_{4}=\mathrm{CH}^{2}(F)_{2} \cdot \mathrm{CH}^{2}(F)_{2} .
$$

Proof. In view of Theorem 2 and Theorem 20.2, it suffices to check that $\mathcal{A}_{\text {hom }} \otimes \mathbb{Q}=L_{*} \mathrm{CH}^{4}(F)$ and that $\operatorname{ker}\left\{I_{*}: \mathrm{CH}^{4}(F) \rightarrow \mathrm{CH}^{2}(F)\right\}=\operatorname{ker}\left\{L_{*}: \mathrm{CH}^{4}(F)_{\text {hom }} \rightarrow \mathrm{CH}^{2}(F)\right\}$. Both equalities follow from the fact proved in Lemma A.5 that $I_{*} \mathfrak{o}_{F}=\frac{1}{3}\left(g^{2}-c\right)$ which is not zero in $\mathrm{H}^{4}(F, \mathbb{Q})$, and from (107) which gives $L_{*} \sigma=\frac{1}{3} \operatorname{deg}(\sigma)\left(g^{2}-c\right)-I_{\sigma}$ for all $\sigma \in \mathrm{CH}^{4}(F)$.

A key step to proving Theorem 20.2(iii) is embodied by Theorem 20.5 ; it consists in giving a different description of the filtration $\mathrm{F}^{\bullet}$ of Definition 20.1. On the one hand, we define

$$
\Phi=p_{*} q^{*}: \mathrm{CH}^{i}(X) \rightarrow \mathrm{CH}^{i-1}(F) \text { and } \Psi=q_{*} p^{*}: \mathrm{CH}^{i}(F) \rightarrow \mathrm{CH}^{i-1}(X)
$$

to be the induced homomorphisms of Chow groups ; see Definition A.2. On the other hand, we introduce the following crucial definition.

Definition 20.4. Three lines $l_{1}, l_{2}, l_{3} \subset X$ form a triangle if there is a linear $\Pi=\mathbb{P}^{2} \subset \mathbb{P}^{5}$ such that $\Pi \cdot X=l_{1}+l_{2}+l_{3}$. Each of the lines in a triangle will be called an edge of the triangle. A line $l \subset X$ is called a triple line if $(l, l, l)$ is a triangle on $X$. Let $\mathcal{R} \subset \mathrm{CH}_{0}^{\mathbb{Z}}(F)=\mathrm{CH}_{\mathbb{Z}}^{4}(F)$ be the sub-group generated by elements of the form $s_{1}+s_{2}+s_{3}$ where $\left(l_{s_{1}}, l_{s_{2}}, l_{s_{3}}\right)$ is a triangle.

Theorem 20.5. Let $\mathcal{R}_{\text {hom }} \subset \mathcal{R}$ be the sub-group of all homologically trivial elements, then

$$
\mathrm{F}^{4} \mathrm{CH}^{4}(F)=\operatorname{ker}\left\{\Psi: \mathrm{CH}^{4}(F) \rightarrow \mathrm{CH}^{3}(X)\right\}=\mathcal{R}_{\text {hom }} .
$$

Proof. To establish the first equality, we note that the composition $\Phi \circ \Psi$ is equal to $I_{*}$. Hence we only need to see that $\Phi: \mathrm{CH}^{3}(X)_{\text {hom }} \rightarrow \mathrm{CH}^{2}(F)_{\text {hom }}$ is injective. This is an immediate consequence of [47, Theorem 4.7] where it is shown that the composition of $\Phi$ and the restriction $\mathrm{CH}^{2}(F)_{\text {hom }} \rightarrow \mathrm{CH}^{2}\left(S_{l}\right)_{\text {hom }}$ is injective for $l$ a general line on $X$.

As for the second equality, the inclusion $\mathcal{R}_{\text {hom }} \subseteq \operatorname{ker}\{\Psi\}$ is obvious and the inclusion $\operatorname{ker}\{\Psi\} \subseteq \mathcal{R}_{\text {hom }}$ is Lemma 20.6 below.

Lemma 20.6. Let $l_{i}$ and $l_{i}^{\prime}, i=1, \ldots, n$, be two collections of lines on $X$ such that $\sum l_{i}=\sum l_{i}^{\prime}$ in $\mathrm{CH}^{3}(X)$. Then we have

$$
\sum_{i=1}^{n}\left[l_{i}\right]-\sum_{i=1}^{n}\left[l_{i}^{\prime}\right] \in \mathcal{R}_{\mathrm{hom}} .
$$


Proof. We recall the concept, which was defined in [46], of a secant line of a pair of (disjoint) curves $C_{1}$ and $C_{2}$ on $X$. Namely $l$ is a secant line of $\left(C_{1}, C_{2}\right)$ if it meets both curves. This concept is naturally generalized to the concept of secant lines of two 1-dimensional cycles on $X$. A pair of curves are wellpositioned if they have finitely many (counted with multiplicities) secant lines. To prove the lemma, we first prove the following.

Claim : Let $\left(l_{1}, l_{2}\right)$ be a pair of well-positioned lines on $X$ and $E_{i}, i=1, \ldots, 5$, the secant lines of $\left(l_{1}, l_{2}\right)$. Then we have

$$
2\left[l_{1}\right]+2\left[l_{2}\right]+\sum_{i=1}^{5}\left[E_{i}\right] \in \mathcal{R} .
$$

Proof of Claim. we may assume that the pair $\left(l_{1}, l_{2}\right)$ is general. This is because the special case follows from the generic case by a limit argument. Let $\Pi \cong \mathbb{P}^{3}$ be the linear span of $l_{1}$ and $l_{2}$. Since $\left(l_{1}, l_{2}\right)$ is general, the intersection $\Sigma=\Pi \cap X \subset \Pi \cong \mathbb{P}^{3}$ is a smooth cubic surface. Hence $\Sigma \cong \mathrm{Bl}_{\left\{P_{1}, \ldots, P_{6}\right\}}\left(\mathbb{P}^{2}\right)$ is the blow-up of $\mathbb{P}^{2}$ at 6 points. Let $R_{i} \subset \Sigma, 1 \leq i \leq 6$, be the the 6 exceptional curves. Let $L_{i j} \subset \Sigma$ be the strict transform of the line on $\mathbb{P}^{2}$ connecting $P_{i}$ and $P_{j}$, where $1 \leq i<j \leq 6$. Let $C_{i} \subset \Sigma, 1 \leq i \leq 6$, be the strict transform of the conic on $\mathbb{P}^{2}$ passing through all $P_{j}$ with $j \neq i$. The set $\left\{R_{i}, L_{i j}, C_{i}\right\}$ gives all the 27 lines on $\Sigma$. Without loss of generality, we may assume that $R_{1}=l_{1}$ and $R_{2}=l_{2}$. Then the set of all secant lines $\left\{E_{i}\right\}_{i=1}^{6}$ is explicitly given by $\left\{L_{12}, C_{3}, C_{4}, C_{5}, C_{6}\right\}$. The triangles on $\Sigma$ are always of the form $\left(R_{i}, L_{i j}, C_{j}\right)$ (here we allow $i>j$ and set $\left.L_{i j}=L_{j i}\right)$ or $\left(L_{i_{1} j_{1}}, L_{i_{2} j_{2}}, L_{i_{3} j_{3}}\right)$ where $\left\{i_{1}, i_{2}, i_{3}, j_{1}, j_{2}, j_{3}\right\}=\{1, \ldots, 6\}$. Then we easily check that

$$
\begin{aligned}
2 l_{1}+2 l_{2}+\sum E_{i}= & 2 R_{1}+2 R_{2}+L_{12}+C_{3}+C_{4}+C_{5}+C_{6} \\
= & \left(R_{1}+L_{13}+C_{3}\right)+\left(R_{1}+L_{14}+C_{4}\right)+\left(R_{2}+L_{25}+C_{5}\right) \\
& +\left(R_{2}+L_{26}+C_{6}\right)+\left(L_{12}+L_{46}+L_{35}\right) \\
& -\left(L_{13}+L_{25}+L_{46}\right)-\left(L_{14}+L_{26}+L_{35}\right) .
\end{aligned}
$$

Hence $2\left[l_{1}\right]+2\left[l_{2}\right]+\sum\left[E_{i}\right] \in \mathcal{R}$.

Back to the proof of the lemma. We pick a general line $l$ such that $\left(l, l_{i}\right)$ and $\left(l, l_{i}^{\prime}\right)$ are all wellpositioned. Let $E_{i, j}, j=1, \ldots, 5$, be the secant lines of $l_{i}$ and $l$; similarly let $E_{i, j}^{\prime}, j=1, \ldots, 5$, be the secant lines of $l_{i}^{\prime}$ and $l$. Then by definition, we have

$$
\sum_{i=1}^{n} \sum_{j=1}^{5}\left[E_{i, j}\right]=\Phi(\gamma) \cdot S_{l}, \quad \sum_{i=1}^{n} \sum_{j=1}^{5}\left[E_{i, j}^{\prime}\right]=\Phi\left(\gamma^{\prime}\right) \cdot S_{l}, \quad \text { in } \mathrm{CH}_{0}(F),
$$

where $\gamma=\sum l_{i} \in \mathrm{CH}_{1}(X)$ and $\gamma^{\prime}=\sum l_{i}^{\prime} \in \mathrm{CH}_{1}(X)$. By assumption $\gamma=\gamma^{\prime}$ and hence

$$
\sum_{i=1}^{n} \sum_{j=1}^{5}\left[E_{i, j}\right]=\sum_{i=1}^{n} \sum_{j=1}^{5}\left[E_{i, j}^{\prime}\right] \text {. }
$$

Thus we get

$$
\begin{aligned}
2\left(\sum_{i=1}^{n}\left[l_{i}\right]-\sum_{i=1}^{5}\left[l_{i}^{\prime}\right]\right) & =\left(2 \sum_{i=1}^{n}\left[l_{i}\right]+2 n[l]+\sum_{i=1}^{n} \sum_{j=1}^{5}\left[E_{i, j}\right]\right)-\left(2 \sum_{i=1}^{n}\left[l_{i}^{\prime}\right]+2 n[l]+\sum_{i=1}^{n} \sum_{j=1}^{5}\left[E_{i, j}^{\prime}\right]\right) \\
& =\sum_{i=1}^{n}\left(2[l]+2\left[l_{i}\right]+\sum_{j=1}^{5}\left[E_{i, j}\right]\right)-\sum_{i=1}^{n}\left(2[l]+2\left[l_{i}^{\prime}\right]+\sum_{j=1}^{5}\left[E_{i, j}^{\prime}\right]\right) \in \mathcal{R}_{\text {hom }} .
\end{aligned}
$$

Here the last step uses the claim. If we can prove that $\mathcal{R}_{\text {hom }}$ is divisible (and hence uniquely divisible), then we get $\sum\left[l_{i}\right]-\sum\left[l_{i}^{\prime}\right] \in \mathcal{R}_{\text {hom. }}$ Let $\widetilde{R}$ be the (desingularized and compactified) moduli space of triangles on $X$, then we have a surjection $\mathrm{CH}_{0}(\widetilde{R})_{\text {hom }} \rightarrow \mathcal{R}_{\text {hom }}$. It is a standard fact that $\mathrm{CH}_{0}(\widetilde{R})_{\text {hom }}$ is divisible. Hence so is $\mathcal{R}_{\text {hom }}$. This finishes the proof.

Proposition 20.7 below will be used to prove Theorem 20.2.

Proposition 20.7. Let $\left(l_{1}, l_{2}, l_{3}\right)$ be a triangle, then the following are true. 
(i) The identity $S_{l_{1}} \cdot S_{l_{2}}=6 \mathfrak{o}_{F}+\left[l_{3}\right]-\left[l_{1}\right]-\left[l_{2}\right]$ holds true in $\mathrm{CH}_{0}(F)$;

(ii) If $\left(l_{1}^{\prime}, l_{2}^{\prime}, l_{3}^{\prime}\right)$ is another triangle, then

$$
6\left(\sum_{i=1}^{i=3}\left[l_{i}\right]-\sum_{i=1}^{3}\left[l_{i}^{\prime}\right]\right)=\sum_{1 \leq i<j \leq 3}\left(S_{l_{i}}-S_{l_{j}}\right)^{2}-\sum_{1 \leq i<j \leq 3}\left(S_{l_{i}^{\prime}}-S_{l_{j}^{\prime}}\right)^{2}
$$

holds true in $\mathrm{CH}_{0}(F)$.

Proof of Proposition 20.7. We prove (i) for the case where all the edges of the triangle are distinct. Then the general case follows by a simple limit argument. Take a 1-dimensional family of lines $\left\{l_{t}: t \in T\right\}$ such that $l_{t_{1}}=l_{1}$. We may assume that $\left(l_{t}, l_{2}\right)$ is well-positioned when $t \neq t_{1}$. Let $\left\{E_{t, i}: i=1, \ldots, 5\right\}$ be the secant lines of the pair $\left(l_{t}, l_{2}\right)$ for $t \neq t_{1}$. The tangent space $\mathscr{T}_{t_{1}}(T)$ determines a section of $\mathrm{H}^{0}\left(l_{1}, \mathscr{N}_{l_{1} / X}\right)$ which in turn gives a normal direction $v$ in $\mathscr{N}_{l_{1} / X, x}$, where $x=l_{1} \cap l_{2}$. When $t \rightarrow t_{1}$, the secant lines $E_{t, i}$ specializes to $\left\{L_{1}, \ldots, L_{4}, l_{3}\right\}$ where $L_{1}, \ldots, L_{4}$ together with $\left\{l_{1}, l_{2}\right\}$ form the six lines through $x$ that are contained in the linear $\mathbb{P}^{3}$ spanned by $\left(l_{1}, l_{2}, v\right)$. This means $\left[L_{1}\right]+\cdots+\left[L_{4}\right]+\left[l_{1}\right]+\left[l_{2}\right]=6 \mathfrak{o}_{F}$; see Lemma A.8. By construction, we have

$$
S_{l_{t}} \cdot S_{l_{2}}=\sum_{i=1}^{5}\left[E_{t, i}\right]
$$

Let $t \rightarrow t_{1}$ and take the limit, we have

$$
S_{l_{1}} \cdot S_{l_{2}}=\sum_{i=1}^{4}\left[L_{i}\right]+\left[l_{3}\right] .
$$

We combine this with $\left[L_{1}\right]+\cdots+\left[L_{4}\right]+\left[l_{1}\right]+\left[l_{2}\right]=6 \mathfrak{o}_{F}$ and deduce (i).

Let $\left(l_{1}, l_{2}, l_{3}\right)$ be a triangle, then we have

$$
\begin{aligned}
\sum_{1 \leq i<j \leq 3}\left(S_{l_{i}}-S_{l_{j}}\right)^{2} & =2\left(\sum_{1 \leq i \leq 3} S_{l_{i}}\right)^{2}-6 \sum_{1 \leq i<j \leq 3} S_{l_{i}} \cdot S_{l_{j}} \\
& =2\left(\Phi\left(h^{3}\right)\right)^{2}+6 \sum_{1 \leq i \leq 3}\left[l_{i}\right]-108 \mathfrak{o}_{F} .
\end{aligned}
$$

Then (ii) follows easily from this computation.

Proof of Theorem 20.2. To prove (ii), it is enough to show that $\left[l_{1}\right]-\left[l_{2}\right]$ is in the image of the map. Let $l^{\prime}$ be a line meeting both $l_{1}$ and $l_{2}$, then $\left[l_{1}\right]-\left[l_{2}\right]=\left(\left[l_{1}\right]-\left[l^{\prime}\right]\right)+\left(\left[l^{\prime}\right]-\left[l_{2}\right]\right)$. Hence we may assume that $l_{1}$ meets $l_{2}$. We may further assume that $\left(l_{1}, l_{2}\right)$ is general. Since $\mathrm{CH}^{4}(F)_{\text {hom }}=\mathrm{CH}_{0}(F)_{\text {hom }}$ is uniquely divisible, we only need to show that $2\left(\left[l_{1}\right]-\left[l_{2}\right]\right)$ is in the image. Now let $l_{3}$ be the residue line of $l_{1} \cup l_{2}$ so that $\left(l_{1}, l_{2}, l_{3}\right)$ forms a triangle. Then

$$
2\left(\left[l_{1}\right]-\left[l_{2}\right]\right)=\left(S_{l_{2}}-S_{l_{1}}\right) \cdot S_{l_{3}},
$$

is in the image of $\mathrm{CH}^{2}(F) \otimes \mathrm{CH}^{2}(F)_{\text {hom }} \rightarrow \mathrm{CH}^{4}(F)_{\text {hom }}$.

To prove (i), it suffices to show that the image of $\mathrm{CH}_{\mathbb{Z}}^{2}(F) \otimes \mathrm{CH}_{\mathbb{Z}}^{2}(F) \rightarrow \mathrm{CH}_{\mathbb{Z}}^{4}(F)$ contains a cycle of degree 1. By Lemma A.3 and the fact that a general pair of lines has 5 secant lines (see [46]), we have

$$
\operatorname{deg}\left(g^{2} \cdot g^{2}\right)=108, \quad \operatorname{deg}\left(S_{l_{1}} \cdot S_{l_{2}}\right)=5
$$

Hence the image of the intersection of 2-cycles hits a 0 -cycle of degree 1.

(iii) As an easy consequence of Proposition 20.7(ii) we have $\mathcal{R}_{\text {hom }}=\mathcal{A}_{\text {hom }} \cdot \mathcal{A}_{\text {hom }}$. But then, Theorem 20.5 gives $\mathrm{F}^{4} \mathrm{CH}^{4}(F)=\mathcal{A}_{\text {hom }} \cdot \mathcal{A}_{\text {hom }}$

Remark 20.8. An alternate proof of statements (i) and (ii) of Theorem 20.2 can be obtained as a combination of Voisin's identity (106) and of the basic Lemma 17.3. However, (106) does not seem to imply statement (iii). In addition, our proofs also work for the case of cubic threefolds as follows. If $X$ is a cubic threefold, then its variety of lines, $S$, is a smooth surface. Then $\mathrm{F}^{2} \mathrm{CH}^{2}(S)$, the Albanese kernel, is the same as $\operatorname{ker}\left\{\Psi: \mathrm{CH}^{2}(S) \rightarrow \mathrm{CH}^{3}(X)\right\}$. As in Theorem $20.5, \mathrm{~F}^{2} \mathrm{CH}^{2}(S)$ is identified with $\mathcal{R}_{\text {hom }}$. Statements (i) and (ii) of Proposition 20.7 are true in the following sense. In this case $S_{l}$, the space of all lines meeting a given line $l$, is a curve. The constant class $6 \mathfrak{o}_{F}$ in statement (i) should be replaced by the 
class of the sum of all lines passing through a general point of $X$. Then the statement (iii) of Theorem 20.2 reads as $\operatorname{Pic}^{0}(S) \otimes \operatorname{Pic}^{0}(S) \rightarrow \mathrm{F}^{2} \mathrm{CH}^{2}(S)$ being surjective. This result concerning the Fano scheme of lines on a smooth cubic threefold already appears in [13].

\section{The Rational Self-MaP $\varphi: F \rightarrow F$ And the Fourier DeComposition}

In this section we determine completely the class in $\mathrm{CH}^{4}(F \times F)$ of the closure of the graph $\Gamma_{\varphi}$ of the rational map $\varphi$ of Definition 18.1; see Proposition 21.2. We obtain thus in Proposition 21.7 a complete description of the cohomology class of $\Gamma_{\varphi}$, thereby complementing the main result of Amerik [2]. After some work, we also obtain in Theorem 21.9 an eigenspace decomposition for the action of $\varphi$ on the Chow groups of $F$. It turns out that the action of $\varphi$ is compatible with the Fourier decomposition on the Chow groups of $F$; see $\S 21.6$. This interplay between $\varphi$ and the Fourier decomposition will be crucial to showing in Section 22 that the Fourier decomposition is compatible with intersection product.

Let $\omega$ be a global 2 form on $F$. The action of $\varphi^{*}$ on $\omega$ is known :

Proposition $21.1([3])$. The rational map $\varphi: F \rightarrow F$ has degree 16 and we have

$$
\varphi^{*} \omega=-2 \omega, \quad \varphi^{*} \omega^{2}=4 \omega^{2} .
$$

By the very definition of $\varphi$, the triple $(l, l, \varphi(l))$ is a triangle, in the sense of Definition 20.4, whenever $l$ is a line of first type. Thus $I_{*}\left(\varphi_{*} \sigma+2 \sigma\right)=0$ for all $\sigma \in \mathrm{CH}_{0}(F)_{\text {hom }}$; see Proposition 20.5. Other links between the rational map $\varphi$ and the incidence correspondence $I$ have already been mentioned : Lemma 18.2 shows that $g^{2} \cdot I_{*}[l]=\varphi_{*}([l])-4[l]+24 \mathfrak{o}_{F} \in \mathrm{CH}_{0}(F)$, and Proposition 20.7 implies, for instance, that $I_{*}[l] \cdot I_{*} \varphi_{*}[l]=6 \mathfrak{o}_{F}-\varphi_{*}[l]$ and $\left(I_{*}[l]\right)^{2}=6 \mathfrak{o}_{F}+\varphi_{*}[l]-2[l]$. Here we wish to relate $I$ and $\varphi$ as correspondences in $F \times F$ and not only the actions of $I$ and $\varphi$ on the Chow groups of $F$. As such, the main result of this section is Proposition 21.2. As a consequence, we determine the action of $\varphi$ on $\mathrm{CH}^{*}(F)$ and study its compatibility with the Fourier transform.

21.1. The correspondence $\Gamma_{\varphi}$. Let $\varphi: F \rightarrow F$ be the rational map introduced in Definition 18.1 and let $\Gamma_{\varphi} \subset F \times F$ be the closure of the graph of $\varphi$. We denote $\Gamma_{\varphi, 0} \subset F \times F$ the set of points $\left(\left[l_{1}\right],\left[l_{2}\right]\right) \in F \times F$ such that there is a linear plane $\mathbb{P}^{2} \subset \mathbb{P}^{5}$, not contained in $X$, with $\mathbb{P}^{2} \cdot X=2 l_{1}+l_{2}$. Then $\Gamma_{\varphi, 0}$ is irreducible and $\Gamma_{\varphi}$ is the closure of $\Gamma_{\varphi, 0}$. Furthermore, if $X$ does not contain any plane, then $\Gamma_{\varphi}=\Gamma_{\varphi, 0}$. Our next goal is to study the correspondence $\Gamma_{\varphi}$ in more details.

Let us now introduce some other natural cycles on $F \times F$; see Appendix A for more details. Let $\Gamma_{h} \subset F \times F$ be the 5 -dimensional cycle of all points $\left(\left[l_{1}\right],\left[l_{2}\right]\right)$ such that $x \in l_{1} \cap l_{2}$ for some point $x$ on a given hyperplane $H \subset X$. Let $\Gamma_{h^{2}} \subset F \times F$ be the 4-dimensional cycle of all points $\left(\left[l_{1}\right],\left[l_{2}\right]\right)$ such that $x \in l_{1} \cap l_{2}$ for some $x$ on an intersection $H_{1} \cap H_{2} \subset X$ of two general hyperplane sections. Let $\Pi_{j} \subset X$, $j=1, \ldots, r$, be all the planes contained in $X$ and $\Pi_{j}^{*} \subset F$ the corresponding dual planes. Then we define $I_{1}=\sum_{j=1}^{r} \Pi_{j}^{*} \times \Pi_{j}^{*} \in \mathrm{CH}^{4}(F \times F)$. Note that a general cubic fourfold does not contain any plane and hence $I_{1}=0$. Now we can state the main result of this section.

Proposition 21.2. The following equation holds true in $\mathrm{CH}^{4}(F \times F)$,

$$
\Gamma_{\varphi}+I_{1}=4 \Delta_{F}+\left(2 g_{1}^{2}+3 g_{1} g_{2}+g_{2}^{2}\right) \cdot I-\left(5 g_{1}+4 g_{2}\right) \cdot \Gamma_{h}+3 \Gamma_{h^{2}} .
$$

Furthermore, $-\left(5 g_{1}+4 g_{2}\right) \cdot \Gamma_{h}+3 \Gamma_{h^{2}}$ can be expressed as a weighted homogeneous polynomial $\Gamma_{2}^{\prime}\left(g_{1}, g_{2}, c_{1}, c_{2}\right)$ of degree 4 ; see Proposition A.6.

Proof. Let $I_{0}=I \backslash \Delta_{F}$. Then we have a natural morphism

$$
q_{0}: I_{0} \rightarrow X, \quad\left(\left[l_{1}\right],\left[l_{2}\right]\right) \mapsto x=l_{1} \cap l_{2} .
$$

Let $\pi_{i}=\left.p_{i}\right|_{I_{0}}: I_{0} \rightarrow F$ be the two projections. We use $\mathscr{E}_{2} \subset V$ to denote the natural rank two sub-bundle of $V$ on $F$ and $\mathscr{E}_{1}=\mathcal{O}_{X}(-1) \subset V$ to denote the restriction of the tautological line bundle. We first note that on $I_{0}$, we have natural inclusions $q_{0}^{*} \mathscr{E}_{1} \hookrightarrow \pi_{i}^{*} \mathscr{E}_{2}, i=1,2$, which correspond to the geometric fact that $x \in l_{i}$. In this way we get the following short exact sequences

$$
0 \longrightarrow q_{0}^{*} \mathscr{E}_{1} \longrightarrow \pi_{i}^{*} \mathscr{E}_{2} \longrightarrow \mathcal{Q}_{i} \longrightarrow 0,
$$


for $i=1,2$, where $\mathcal{Q}_{i}$ is an invertible sheaf on $I_{0}$. Let $\mathcal{V}_{3} \subset V$ be the rank 3 sub-bundle spanned by $\pi_{1}^{*} \mathscr{E}_{2}$ and $\pi_{2}^{*} \mathscr{E}_{2}$, then we have the following short exact sequence

$$
0 \longrightarrow q_{0}^{*} \mathscr{E}_{1} \stackrel{(+,-)}{\longrightarrow} \pi_{1}^{*} \mathscr{E}_{2} \oplus \pi_{2}^{*} \mathscr{E}_{2} \longrightarrow \mathcal{V}_{3} \longrightarrow 0
$$

At a point $\left(\left[l_{1}\right],\left[l_{2}\right]\right) \in I_{0}$, the fiber of $\mathcal{V}_{3}$ is the 3 -dimensional sub-space of $V$ that corresponds to the linear $\mathbb{P}^{2}$ spanned by $l_{1}$ and $l_{2}$. We have a natural inclusion $\pi_{i}^{*} \mathscr{E}_{2} \subset \mathcal{V}_{3}$ whose quotient is isomorphic to $\mathcal{Q}_{j}$ for $\{i, j\}=\{1,2\}$. This gives a natural surjection $\mathcal{V}_{3} \rightarrow \mathcal{Q}_{1} \oplus \mathcal{Q}_{2}$ which fits into the following short exact sequence

$$
0 \longrightarrow q_{0}^{*} \mathscr{E}_{1} \longrightarrow \mathcal{V}_{3} \longrightarrow \mathcal{Q}_{1} \oplus \mathcal{Q}_{2} \longrightarrow 0
$$

We write $\mathscr{F}_{i}=\pi_{i}^{*} \mathscr{E}_{2}, i=1,2$, and note that we have natural inclusions $\mathscr{F}_{i} \subset \mathcal{V}_{3}$. By taking the third symmetric power, we get

$$
0 \longrightarrow \mathscr{F}_{0} \longrightarrow \operatorname{Sym}^{3} \mathcal{V}_{3} \longrightarrow \mathscr{G} \longrightarrow 0,
$$

where $\mathscr{F}_{0}=\frac{\operatorname{Sym}^{3} \mathscr{F}_{1} \oplus \mathrm{Sym}^{3} \mathscr{F}_{2}}{q_{0}^{*} \mathscr{E}_{1}^{\otimes 3}}$ and $\mathscr{G}$ is locally free of rank 3 on $I_{0}$. Let $\phi=0$, for some $\phi \in \operatorname{Sym}^{3}(V)^{\vee}$, be the defining equation of $X \subset \mathbb{P}(V)$. Then by restricting to the sub-bundle $\operatorname{Sym}^{3} \mathcal{V}_{3}$ of $\operatorname{Sym}^{3} V$, we get a homomorphism $\phi_{1}: \operatorname{Sym}^{3} \mathcal{V}_{3} \rightarrow \mathcal{O}_{I_{0}}$. Note that at a closed point $\left(\left[l_{1}\right],\left[l_{2}\right]\right) \in I_{0}, \mathscr{F}_{i} \subset V$ corresponds to the line $l_{i}, i=1,2$. The fact that $l_{i}$ is contained in $X$ implies that $\phi_{1}$ vanishes on $\operatorname{Sym}^{3} \mathscr{F}_{i}$. Hence by the short exact sequence (112), we see that $\phi_{1}$ induces a homomorphism

$$
\phi_{2}: \mathscr{G} \longrightarrow \mathcal{O}_{I_{0}} \text {. }
$$

Consider the following diagram

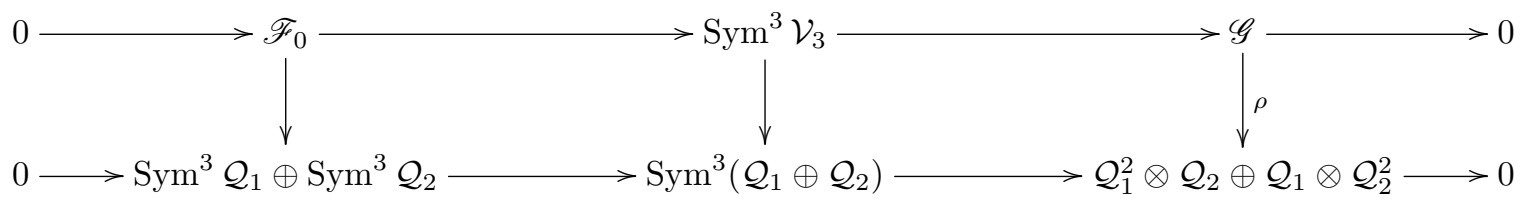

where all the vertical arrows are surjections. The homomorphism $\rho$ fits into the following short exact sequence

$$
0 \longrightarrow q_{0}^{*} \mathscr{E}_{1} \otimes \mathcal{Q}_{1} \otimes \mathcal{Q}_{2} \longrightarrow \mathscr{G} \longrightarrow \mathcal{Q}_{1}^{2} \otimes \mathcal{Q}_{2} \oplus \mathcal{Q}_{1} \otimes \mathcal{Q}_{2}^{2} \longrightarrow 0
$$

Hence $\phi_{2}$ induces an homomorphism

$$
\phi_{3}: q_{0}^{*} \mathscr{E}_{1} \otimes \mathcal{Q}_{1} \otimes \mathcal{Q}_{2} \longrightarrow \mathcal{O}_{I_{0}} .
$$

Let $Z \subset I_{0}$ be the locus defined by $\phi_{3}=0$. Hence on $Z$ the homomorphism $\phi_{3}$ factors through the third term in the sequence (113) and gives

$$
\phi_{4}: \mathcal{Q}_{1}^{2} \otimes \mathcal{Q}_{2} \oplus \mathcal{Q}_{1} \otimes \mathcal{Q}_{2}^{2} \longrightarrow \mathcal{O}_{I_{0}} .
$$

This homomorphism further splits as $\phi_{4}=\phi_{4,1}+\phi_{4,2}$, where

$$
\begin{aligned}
\phi_{4,1}: \mathcal{Q}_{1}^{2} \otimes \mathcal{Q}_{2} \longrightarrow \mathcal{O}_{I_{0}}, \\
\phi_{4,2}: \mathcal{Q}_{1} \otimes \mathcal{Q}_{2}^{2} \longrightarrow \mathcal{O}_{I_{0}} .
\end{aligned}
$$

Let $Z^{\prime} \subset Z$ be the locus defined by $\phi_{4,1}=0$. The cycle class of $Z^{\prime}$ on $I_{0}$ can then be computed as

$$
\begin{aligned}
Z^{\prime} & =c_{1}\left(q_{0}^{*} \mathscr{E}_{1}^{-1} \otimes \mathcal{Q}_{1}^{-1} \otimes \mathcal{Q}_{2}^{-1}\right) \cdot c_{1}\left(\mathcal{Q}_{1}^{-2} \otimes \mathcal{Q}_{2}^{-1}\right) \\
& =\left(q_{0}^{*} h+\left.g_{1}\right|_{I_{0}}-q_{0}^{*} h+\left.g_{2}\right|_{I_{0}}-q_{0}^{*} h\right)\left(\left.2 g_{1}\right|_{I_{0}}-2 q_{0}^{*} h+\left.g_{2}\right|_{I_{0}}-q_{0}^{*} h\right) \\
& =\left.\left(2 g_{1}^{2}+3 g_{1} g_{2}+g_{2}^{2}\right)\right|_{I_{0}}-\left(5 g_{1}+4 g_{2}\right) \cdot q_{0}^{*} h+3 q_{0}^{*} h^{2} .
\end{aligned}
$$

Here the second equality uses the fact that $-c_{1}\left(\mathcal{Q}_{i}\right)=\left.g_{i}\right|_{I_{0}}-q_{0}^{*} h, i=1,2$, which is a simple consequence of the sequence (110). Let $i_{0}: I_{0} \rightarrow F \times F \backslash \Delta_{F}$ be the inclusion, then we have

$$
\left(i_{0}\right)_{*} Z^{\prime}=\left(2 g_{1}^{2}+3 g_{1} g_{2}+g_{2}^{2}\right) \cdot I-\left(5 g_{1}+4 g_{2}\right) \cdot \Gamma_{h}+3 \Gamma_{h^{2}}, \quad \text { on } F \times F \backslash \Delta_{F} .
$$


Our next step is to relate $Z^{\prime}$ to $\Gamma_{\varphi}$. Let $e_{0}$ (resp. $e_{1}$ and $e_{2}$ ) be a local generator of $q_{0}^{*} \mathscr{E}_{1}$ (resp. $\mathcal{Q}_{1}$ and $\mathcal{Q}_{2}$ ) and $T_{0}$ (resp. $T_{1}$ and $T_{2}$ ) be the dual generator of $q_{0}^{*} \mathscr{E}_{1}^{-1}$ (resp. $\mathcal{Q}_{1}^{-1}$ and $\mathcal{Q}_{2}^{-1}$ ). Then $\left(T_{0}, T_{1}, T_{2}\right)$ can be viewed as homogeneous coordinates of the plane $\Pi_{t}$ spanned by $l_{1}$ and $l_{2}$, where $t=\left(\left[l_{1}\right],\left[l_{2}\right]\right) \in I_{0}$ is a point at which the $T_{i}$ 's are defined. Furthermore, $l_{1}$ is defined by $T_{2}=0$ and $l_{2}$ is defined by $T_{1}=0$. Let $\phi_{t}$ be the restriction of $\phi$ to $\Pi_{t}$. The fact that $\phi=0$ along $l_{1}$ and $l_{2}$ implies that $\phi_{t}=a T_{0} T_{1} T_{2}+b T_{1}^{2} T_{2}+c T_{1} T_{2}^{2}$. The condition $\phi_{3}=0$ is equivalent to $a=0$ and the condition $\phi_{4,1}=0$ is equivalent to $b=0$. Thence $t \in Z^{\prime}$ if and only if $\phi_{t}=c T_{1} T_{2}^{2}$ for some constant $c$. Equivalently, we have

$$
Z^{\prime}=\left\{t \in I_{0}: \Pi_{t} \cdot X=2 l_{1}+l_{2} \text { or } \Pi_{t} \subset X\right\} .
$$

It follows that $\left(i_{0}\right)_{*} Z^{\prime}=\Gamma_{\varphi}+I_{1}$ on $F \times F \backslash \Delta_{F}$. Using the localization sequence for Chow groups and equation (114), we get

$$
\Gamma_{\varphi}+I_{1}=\alpha \Delta_{F}+\left(2 g_{1}^{2}+3 g_{1} g_{2}+g_{2}^{2}\right) \cdot I-\left(5 g_{1}+4 g_{2}\right) \cdot \Gamma_{h}+3 \Gamma_{h^{2}}, \quad \text { in } \mathrm{CH}^{4}(F \times F),
$$

for some integer $\alpha$. By Proposition A.6, the terms involving $\Gamma_{h}$ and $\Gamma_{h^{2}}$ are polynomials in $g_{i}$ and $c_{i}$. To determine the value of $\alpha$, we note that all the terms in the above formula act trivially on $\mathrm{H}^{4,0}(F)$

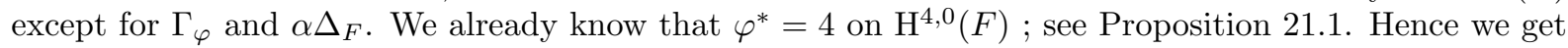
$\alpha=4$.

21.2. The action of $\varphi$ on homologically trivial cycles. Let $\Gamma_{\varphi} \subset F \times F$ be the closure of the graph of $\varphi$ as before. Then, for any element $\sigma \in \mathrm{CH}^{i}(F)$, we define

$$
\varphi^{*} \sigma=p_{1 *}\left(\Gamma_{\varphi} \cdot p_{2}^{*} \sigma\right) \quad \text { and } \quad \varphi_{*} \sigma=p_{2 *}\left(\Gamma_{\varphi} \cdot p_{1}^{*} \sigma\right)
$$

See Appendix B for a general discussion on the action of rational maps on Chow groups. The main goal of this section is to get explicit descriptions of $\varphi^{*}$ and $\varphi_{*}$.

Proposition 21.3. The following are true.

(i) The action of $\varphi^{*}$ on homologically trivial cycles can be described by

$$
\begin{aligned}
& \varphi^{*} \sigma=4 \sigma+2 g^{2} \cdot I_{*} \sigma, \quad \sigma \in \mathrm{CH}^{4}(F)_{\mathrm{hom}} \\
& \varphi^{*} \sigma=4 \sigma+3 g \cdot I_{*}(g \cdot \sigma), \quad \sigma \in \mathrm{CH}^{3}(F)_{\mathrm{hom}} \\
& \varphi^{*} \sigma=4 \sigma+I_{*}\left(g^{2} \cdot \sigma\right), \quad \sigma \in \mathrm{CH}^{2}(F)_{\text {hom }} ;
\end{aligned}
$$

(ii) The action of $\varphi_{*}$ on homologically trivial cycles can be described by

$$
\begin{aligned}
& \varphi_{*} \sigma=4 \sigma+g^{2} \cdot I_{*} \sigma, \quad \sigma \in \mathrm{CH}^{4}(F)_{\mathrm{hom}} \\
& \varphi_{*} \sigma=4 \sigma+3 g \cdot I_{*}(g \cdot \sigma), \quad \sigma \in \mathrm{CH}^{3}(F)_{\mathrm{hom}} \\
& \varphi_{*} \sigma=4 \sigma+2 I_{*}\left(g^{2} \cdot \sigma\right), \quad \sigma \in \mathrm{CH}^{2}(F)_{\mathrm{hom} .}
\end{aligned}
$$

Proof. In view of Proposition 21.2, (i) and (ii) are direct consequences of the following three facts. First, since $I_{1}$ is supported on the union of the $\Pi_{j}^{*} \times \Pi_{j}^{*}$, the action of $I_{1}$ on $\mathrm{CH}^{i}(F)$ factors through both $\bigoplus_{j} \mathrm{CH}_{i}\left(\Pi_{j}^{*}\right)$ and $\bigoplus_{j} \mathrm{CH}^{i}\left(\Pi_{j}^{*}\right)$. Therefore, $I_{1}$ acts as zero on $\mathrm{CH}^{i}(F)$ for $i \neq 2$ and since $\mathrm{CH}^{*}\left(\Pi_{j}^{*}\right)_{\text {hom }}=0$ it also acts as zero on $\mathrm{CH}^{*}(F)_{\text {hom }}$. Secondly, the action of $p_{1}^{*} \alpha \cdot p_{2}^{*} \beta \cdot \Gamma$ on $\sigma \in \mathrm{CH}^{*}(F)$, where $\alpha, \beta \in$ $\mathrm{CH}^{*}(F)$ and $\Gamma \in \mathrm{CH}^{*}(F \times F)$, is given by

$$
\left(p_{1}^{*} \alpha \cdot p_{2}^{*} \beta \cdot \Gamma\right)^{*} \sigma=\alpha \cdot \Gamma^{*}(\beta \cdot \sigma) .
$$

Thirdly, if $\Gamma_{2}^{\prime} \in \mathrm{CH}^{*}(F \times F)$ is a polynomial in the variables $c_{1}, c_{2}, g_{1}, g_{2}$, then for $\sigma \in \mathrm{CH}^{*}(F)_{\text {hom }}$ $\left(\Gamma_{2}^{\prime}\right)^{*} \sigma \in \mathrm{CH}^{*}(F)$ is a polynomial in the variables $c, g$ which is homologically trivial. The main result of [54] recalled in Theorem 4.3 implies that $\left(\Gamma_{2}^{\prime}\right)^{*} \sigma=0$ in $\mathrm{CH}^{*}(F)$.

21.3. The action of $\varphi$ on $g, c$ and their products. Amerik [2] computed, for $X$ generic, the action of $\varphi^{*}$ on the powers of $g$ and on $c$. We use Proposition 21.2 to complement Amerik's result.

Proposition 21.4. We have $\varphi^{*} g=7 g, \varphi_{*} g=28 g, \varphi^{*} g^{2}=\varphi_{*} g^{2}=4 g^{2}+45 c-I_{1}^{*} g^{2}, \varphi^{*} c=\varphi_{*} c=$ $31 c-I_{1}^{*} c, \varphi^{*} g^{3}=28 g^{3}$ and $\varphi_{*} g^{3}=7 g^{3}$. 
Proof. First note, as in the proof of Proposition 21.2, that $I_{1}$ acts as zero on $\mathrm{CH}^{i}(F)$ for $i \neq 2$ but also that the correspondence $I_{1}$ is self-transpose. To prove $\varphi_{*} g=28 g$, we apply the identity in Proposition 21.2 and Lemmas A.3 and A.4. We have

$$
\begin{aligned}
\varphi_{*} g & =4 g+2 I_{*} g^{3}+3 g \cdot I_{*} g^{2}-4 g \cdot\left(\Gamma_{h}\right)_{*} g-5\left(\Gamma_{h}\right)_{*} g^{2}+3\left(\Gamma_{h^{2}}\right)_{*} g \\
& =4 g+72 g+63 g-4 \cdot 6 g-5 \cdot 21 g+3 \cdot 6 g
\end{aligned}
$$

One simplifies the above expression and gets $\varphi_{*} g=28 g$. Let us now compute $\varphi_{*} c$. By Proposition 21.2, we have

$$
\varphi_{*} c+I_{1}^{*} c=4 c+2 I_{*}\left(g^{2} \cdot c\right)+3 g \cdot I_{*}(g \cdot c)+g^{2} \cdot I_{*} c-5\left(\Gamma_{h}\right)_{*}(g \cdot c)-4 g \cdot\left(\Gamma_{h}\right)_{*} c+3\left(\Gamma_{h^{2}}\right)_{*} c .
$$

Lemma A.3 and Proposition A.6 give $\left(\Gamma_{h}\right)_{*} c=6 g$ and $\left(\Gamma_{h^{2}}\right)_{*} c=6\left(g^{2}-c\right)$. On the other hand, [54, Lemma 3.5] shows that $g \cdot c$ and $g^{3}$ are proportional. Since by Lemma A.3 $\operatorname{deg}\left(g^{2} \cdot c\right)=45$ and $\operatorname{deg}\left(g^{4}\right)=108$, we get $12 g \cdot c=5 g^{3}$. In order to finish off the computation of $\varphi_{*} c$ using Lemma A.4, it remains to compute $I_{*} c$. Since $6 l=25 g^{2}-40 c$ and $L_{*} l=0$, we get $8 L_{*} c=5 L_{*} g^{2}$. Now by (107), $L=\frac{1}{3}\left(g_{1}^{2}+\frac{3}{2} g_{1} g_{2}+g_{2}^{2}-c_{1}-c_{2}\right)-I$. A straightforward calculation yields $I_{*} c=6[F]$. Putting all together gives the required $\varphi_{*} c=31 c-I_{1}^{*} c$. The other identities can be proved by using the same basic arguments and are therefore omitted.

21.4. Eigenspace decomposition of the cohomology groups. Let us denote $\tau: \Gamma_{\varphi} \rightarrow F$ and $\widetilde{\varphi}: \Gamma_{\varphi} \rightarrow F$ the first and second projection restricted to $\Gamma_{\varphi} \subset F \times F$. Thus $\varphi^{*} \sigma=\tau_{*} \widetilde{\varphi}^{*} \sigma$ and $\varphi_{*} \sigma=\widetilde{\varphi}_{*} \tau^{*} \sigma$ for all $\sigma \in \mathrm{CH}^{*}(F)$. Let us also denote $\Pi_{1}, \ldots, \Pi_{n}$ the planes contained in $X, \Pi_{1}^{*}, \ldots, \Pi_{n}^{*}$ the corresponding dual planes contained in $F$ and $E_{1}, \ldots, E_{n}$ their pre-images under $\tau$. Finally, we write $E$ for the ruled divisor which is the pre-image of the surface $\Sigma_{2}$ of lines of second type on $F$; see Lemma A.9. The Chow group $\mathrm{CH}^{1}(F)$ splits as

$$
\mathrm{CH}^{1}(F)=\langle g\rangle \oplus \mathrm{CH}^{1}(F)_{\text {prim }},
$$

where $\mathrm{CH}^{1}(F)_{\text {prim }}:=p_{*} q^{*} \mathrm{CH}_{2}(X)_{\text {prim }}$ and $\mathrm{CH}_{2}(X)_{\text {prim }}:=\left\{Z \in \mathrm{CH}_{2}(X):[Z] \in \mathrm{H}^{4}(X, \mathbb{Q})_{\text {prim }}\right\}$. An easy adaptation of [2, Proposition 6] and [54, Lemma 3.11] is the following.

Lemma 21.5. We have $\widetilde{\varphi}^{*} g=7 \tau^{*} g-3 E+\sum_{i} a_{g, i} E_{i}$ for some numbers $a_{g, i} \in \mathbb{Z}$, and for $D \in \mathrm{CH}^{1}(F)_{\text {prim }}$ we have $\widetilde{\varphi}^{*} D=-2 \tau^{*} D+\sum_{i} a_{D, i} E_{i}$ for some numbers $a_{D, i} \in \mathbb{Z}$.

Remark 21.6. Note that by Proposition 21.4, $\varphi_{*} g=28 \mathrm{~g}$, so that if $X$ does not contain any plane we get $7 \cdot 28 g=7 \cdot \varphi_{*} g=\widetilde{\varphi}_{*} \widetilde{\varphi}^{*} g+3 \widetilde{\varphi}_{*} E=16 g+3 \widetilde{\varphi}_{*} E$ and thus $\widetilde{\varphi}_{*} E=4 g$. Likewise, one may compute $3 \widetilde{\varphi}_{*} E^{2}=52 g^{2}+735 c$ and $4 \widetilde{\varphi}_{*} E^{3}=8059 g^{3}$.

First we have the following cohomological description of $\Gamma_{\varphi}$. Note that Proposition 21.2, together with (107), already fully computed the cohomology class of $\Gamma_{\varphi}$ in terms of $g, c$ and the Beauville-Bogomolov class $\mathfrak{B}$.

Proposition 21.7. Let $X$ be a smooth cubic fourfold and let $F$ be its variety of lines. Then

- $\left(\varphi^{*}+2\right)\left(\varphi^{*}-7\right)$ vanishes on $\mathrm{H}^{2}(F, \mathbb{Q})$;

- $\left(\varphi^{*}-28\right)\left(\varphi^{*}+8\right)$ vanishes on $\mathrm{H}^{6}(F, \mathbb{Q})$.

Assume moreover that $X$ does not contain any plane. Then

- $\left(\varphi^{*}-31\right)\left(\varphi^{*}+14\right)\left(\varphi^{*}-4\right)$ vanishes on $\mathrm{H}^{4}(F, \mathbb{Q})$.

Proof. According to Proposition 21.2, we know that the cohomology class of $\Gamma_{\varphi}+I_{1}$ is constant in the family, where $I_{1}=0$ if $X$ does not contain any plane. As in the proof of Proposition 21.3, we see that $I_{1}$ acts trivially on $\mathrm{H}^{i}(F, \mathbb{Q})$ unless possibly when $i=4$. Therefore it is enough to prove the proposition for generic $X$. In particular $X$ does not contain any plane and, as explained in [2, Remark 9], $\mathrm{H}^{2}(F, \mathbb{Q})$ decomposes as $\langle g\rangle \oplus \mathrm{H}^{2}(F, \mathbb{Q})_{\text {prim }}$, where $\mathrm{H}^{2}(F, \mathbb{Q})_{\text {prim }}$ is a simple Hodge structure spanned by a nowhere degenerate global 2-form $\omega$, and $\mathrm{H}^{4}(F, \mathbb{Q})=\operatorname{Sym}^{2} \mathrm{H}^{2}(F, \mathbb{Q})$ decomposes as a direct sum of simple Hodge structures

$$
\mathrm{H}^{4}(F, \mathbb{Q})=\left\langle g^{2}\right\rangle \oplus\langle c\rangle \oplus\left(g \cdot \mathrm{H}^{2}(F, \mathbb{Q})_{\operatorname{prim}}\right) \oplus V,
$$

where $V$ is spanned by $\omega^{2}$.

By Proposition 21.1 and Proposition 21.3 respectively, we see that $\varphi^{*}$ acts by multiplication by -2 on $\mathrm{H}^{2}(F, \mathbb{Q})_{\text {prim }}$ and by multiplication by 7 on $\langle g\rangle$. Thus $\left(\varphi^{*}+2\right)\left(\varphi^{*}-7\right)$ vanishes on $\mathrm{H}^{2}(F, \mathbb{Q})$. By 
MINGMIN SHEN AND CHARLES VIAL

Proposition 21.1, $\varphi^{*}-4$ vanishes on $V$. By Proposition 21.4, $\varphi^{*} g^{2}=4 g^{2}+45 c$ and $\varphi^{*} c=31 c$. Since clearly $\widetilde{\varphi}^{*} \omega=-2 \tau^{*} \omega$, it is straightforward from the projection formula to see that $\varphi^{*}([g] \cup \omega)=-14[g] \cup \omega$. Therefore $\left(\varphi^{*}-31\right)\left(\varphi^{*}+14\right)\left(\varphi^{*}-4\right)$ vanishes on $\mathrm{H}^{4}(F, \mathbb{Q})$. Finally, by the Lefschetz isomorphism, we have $\mathrm{H}^{6}(F, \mathbb{Q})=g^{2} \cdot \mathrm{H}^{2}(F, \mathbb{Q})=\left\langle g^{3}\right\rangle \oplus g^{2} \cdot \mathrm{H}^{2}(F, \mathbb{Q})_{\text {prim }}$. By [2, Theorem 8], $\varphi^{*} g^{3}=28 g^{3}$. It remains to compute $\varphi^{*}\left([g]^{2} \cup \omega\right)$. We have

$\varphi^{*}\left([g]^{2} \cup \omega\right)=\tau_{*}\left(\widetilde{\varphi}^{*}[g]^{2} \cup(-2) \tau^{*} \omega\right)=\varphi^{*}[g]^{2} \cup(-2 \omega)=\left(4[g]^{2}+45[c]\right) \cup(-2 \omega)=-8[g]^{2} \cup \omega-90[c] \cup \omega$.

But then, for generic $X, c$ can be represented by a singular rational surface ; see the proof of Lemma 3.2 in [54]. Therefore $[c] \cup \omega=0$ and hence $\varphi^{*}\left([g]^{2} \cup \omega\right)=-8[g]^{2} \cup \omega$.

21.5. Eigenspace decomposition of the Chow groups. In this paragraph we show that for a cubic fourfold $X$ not containing any plane, the Chow groups of its variety of lines $F$ split under the action of $\varphi^{*}$. Let us fix some notations.

Definition 21.8. For $\lambda \in \mathbb{Q}$, we set

$$
V_{\lambda}^{i}:=\left\{\sigma \in \mathrm{CH}^{i}(F): \varphi^{*} \sigma=\lambda \sigma\right\} .
$$

For instance, $\mathrm{CH}^{0}(F)=V_{1}^{4}$. According to Proposition 21.7 we have

$$
\mathrm{CH}^{1}(F)=V_{7}^{1} \oplus V_{-2}^{1} \text {, with } V_{7}^{1}=\langle g\rangle \text { and } V_{-2}^{1}=\mathrm{CH}^{1}(F)_{\text {prim }} .
$$

The following is the main theorem of this section.

Theorem 21.9. Let $X$ be a smooth cubic fourfold and let $F$ be its variety of lines. Let $\varphi: F \rightarrow F$ be the rational map of Definition 18.1. Then the following are true.

(i) The action of $\varphi^{*}$ on $\mathrm{CH}^{4}(F)$ satisfies $\left(\varphi^{*}-16\right)\left(\varphi^{*}+8\right)\left(\varphi^{*}-4\right)=0$ and induces an eigenspace decomposition

$$
\mathrm{CH}^{4}(F)=V_{16}^{4} \oplus V_{-8}^{4} \oplus V_{4}^{4},
$$

with $\quad \mathrm{CH}^{4}(F)_{0}=V_{16}^{4}, \quad \mathrm{CH}^{4}(F)_{2}=V_{-8}^{4}, \quad \mathrm{CH}^{4}(F)_{4}=V_{4}^{4}$.

(ii) The action of $\varphi^{*}$ on $\mathrm{CH}^{3}(F)$ satisfies $\left(\varphi^{*}-28\right)\left(\varphi^{*}+8\right)\left(\varphi^{*}-4\right)\left(\varphi^{*}+14\right)=0$ and induces a decomposition

$$
\begin{gathered}
\mathrm{CH}^{3}(F)=V_{28}^{3} \oplus V_{-8}^{3} \oplus V_{4}^{3} \oplus V_{-14}^{3}, \\
\text { with } V_{28}^{3}=\left\langle g^{3}\right\rangle, \quad V_{-8}^{3}=g^{2} \cdot V_{-2}^{1}, \quad V_{-14}^{3}=g \cdot V_{-2}^{2}, \\
\mathrm{CH}^{3}(F)_{0}=V_{28}^{3} \oplus V_{-8}^{3}, \quad \mathrm{CH}^{3}(F)_{2}=V_{4}^{3} \oplus V_{-14}^{3} .
\end{gathered}
$$

(iii) The action of $\varphi^{*}$ on $\mathrm{CH}^{2}(F)$ satisfies $\left(\varphi^{*}-31\right)\left(\varphi^{*}+14\right)\left(\varphi^{*}-4\right)\left(\varphi^{*}+2\right)=0$, provided that $X$ does not contain any plane, and induces a decomposition

$$
\begin{gathered}
\mathrm{CH}^{2}(F)=V_{31}^{2} \oplus V_{-14}^{2} \oplus V_{4}^{2} \oplus V_{-2}^{2}, \\
\text { with } \quad V_{31}^{2}=\langle c\rangle, \quad V_{-14}^{2}=g \cdot V_{-2}^{1}, \\
\mathrm{CH}^{2}(F)_{2}=V_{-2}^{2}, \quad \mathrm{CH}^{2}(F)_{0}=V_{31}^{2} \oplus V_{-14}^{2} \oplus V_{4}^{2} .
\end{gathered}
$$

That $\mathrm{CH}^{2}(F)_{2}$ is an eigenspace for the action of $\varphi^{*}$ on $\mathrm{CH}^{2}(F)$ is singled out in the following proposition. Its proof uses the triangle relation introduced in Definition 20.4, and its link to the filtration $\mathrm{F}^{\bullet}$ on $\mathrm{CH}^{4}(F)$ as shown in Theorem 20.5.

Proposition 21.10. Let $F$ be the variety of lines on a cubic fourfold. Then, inside $\mathrm{CH}^{2}(F)$, the sub-group $V_{-2}^{2}$ coincides with the sub-group $\mathcal{A}_{\text {hom }}$ introduced in Definition 20.1. In particular, $V_{-2}^{2}=\mathrm{CH}^{2}(F)_{2}$.

Proof. The group $\mathcal{A}_{\text {hom }}$ is spanned by cycles of the form $S_{l_{1}}-S_{l_{2}}$. Let us compute $\varphi^{*} \sigma$ for $\sigma=S_{l_{1}}-S_{l_{2}}=$ $I_{*} x$ where $x=\left[l_{1}\right]-\left[l_{2}\right] \in \mathrm{CH}_{0}(F)$.

$$
\begin{aligned}
\varphi^{*} \sigma & =4 \sigma+I_{*}\left(g^{2} \cdot I_{*} x\right) \\
& =4 \sigma+I_{*}\left(\varphi_{*} x-4 x\right) \\
& =4 \sigma+I_{*}\left(\varphi_{*} x+2 x\right)-I_{*}(6 x) \\
& =4 \sigma-6 \sigma \\
& =-2 \sigma .
\end{aligned}
$$


Thus $\mathcal{A}_{\text {hom }} \subseteq V_{-2}^{2}$. Here, the first and second equalities are Proposition 21.3. The fourth equality follows simply from the fact that $\varphi_{*} x+2 x$ satisfies the triangle relation of Definition 20.4, i.e., belongs to $\mathcal{R}_{\text {hom }}$, and that $I_{*}$ acts as zero on $\mathcal{R}_{\text {hom }}$ by Theorem 20.5 .

Consider now $\sigma \in \mathrm{CH}^{2}(F)$ such that $\varphi^{*} \sigma=-2 \sigma$. Because -2 is not an eigenvalue of $\varphi^{*}$ acting on $\mathrm{H}^{4}(F, \mathbb{Q})$, we see that $\sigma \in \mathrm{CH}^{2}(F)_{\text {hom. }}$ By Proposition 21.3 , we have $\varphi^{*} \sigma=4 \sigma+I_{*}\left(g^{2} \cdot \sigma\right)$ so that $I_{*}\left(g^{2} \cdot \sigma\right)=-6 \sigma$. Thus $6 V_{-2}^{2} \subseteq I_{*} \mathrm{CH}_{0}(F)_{\text {hom }}:=\mathcal{A}_{\text {hom }}$.

Proof of Theorem 21.9. We first show (i), the decomposition for $\mathrm{CH}^{4}(F)$. Let $x \in \mathrm{CH}^{4}(F)_{\text {hom }}$, then $\varphi_{*} x+2 x \in \mathcal{R}_{\text {hom }}$. Proposition 20.5 yields $I_{*}\left(\varphi_{*} x+2 x\right)=0$. Hence by Proposition 21.3 we have

$$
\left(\varphi_{*}-4\right)\left(\varphi_{*}+2\right) x=g^{2} \cdot I_{*}\left(\varphi_{*} x+2 x\right)=g^{2} \cdot 0=0 .
$$

This implies that $\left(\varphi^{*}-4\right)\left(\varphi^{*}+8\right)=0$ on $\mathrm{CH}^{4}(F)_{\text {hom. }}$. The rational map $\varphi$ has degree 16 and hence, by Proposition B.7, $\varphi_{*} \varphi^{*}=16$ on $\mathrm{CH}^{4}(F)$. If $x \in \mathrm{CH}^{4}(F)$, then $\varphi^{*} x-16 x \in \mathrm{CH}^{4}(F)_{\text {hom }}$ and hence $\left(\varphi^{*}-4\right)\left(\varphi^{*}+8\right)\left(\varphi^{*}-16\right) x=0$. This establishes the polynomial equation that $\varphi^{*}$ satisfies on $\mathrm{CH}^{4}(F)$. Let us prove that $\varphi^{*} \mathfrak{o}_{F}=16 \mathfrak{o}_{F}$, or equivalently $\varphi_{*} \mathfrak{o}_{F}=\mathfrak{o}_{F}$. This will show that $V_{0}^{16}=\mathrm{CH}^{4}(F)_{0}$ and that $\mathrm{CH}^{4}(F)_{\text {hom }}=V_{-8}^{4} \oplus V_{4}^{4}$. By Lemma A.5, we have $I_{*} \mathfrak{o}_{F}=\frac{1}{3}\left(g^{2}-c\right)$. Meanwhile, having in mind that whenever $\varphi$ is defined at $l$ the triple $(l, l, \varphi(l))$ is a triangle, Proposition 20.7(i) yields $\left(I_{*} \mathfrak{o}_{F}\right)^{2}=\varphi_{*} \mathfrak{o}_{F}+4 \mathfrak{o}_{F}$. Hence using the identities in (i) of Lemma A.3, we get

$$
\varphi_{*} \mathfrak{o}_{F}+4 \mathfrak{o}_{F}=\frac{1}{9}\left(g^{4}-2 g^{2} c+c^{2}\right)=5 \mathfrak{o}_{F} .
$$

It follows that $\varphi_{*} \mathfrak{o}_{F}=\mathfrak{o}_{F}$.

Finally, we have $g^{2} \cdot I_{*}=\varphi_{*}-4=-6$ on $V_{-8}^{4}$; see Proposition 21.3. This implies that $I_{*}$ induces an isomorphism $V_{-8}^{4} \stackrel{\cong}{\longrightarrow} \mathcal{A}_{\text {hom }}$ and that $\mathrm{F}^{4} \mathrm{CH}^{4}(F)=\operatorname{ker}\left(I_{*}\right)=V_{4}^{4}$. This shows that $V_{-8}^{4}=\mathrm{CH}^{4}(F)_{2}$ and $V_{0}^{4}=\mathrm{CH}^{4}(F)_{4}$.

Now we show (iii), the decomposition for $\mathrm{CH}^{2}(F)$ when $X$ does not contain any plane. For $\sigma \in \mathrm{CH}^{2}(F)$, Proposition 21.2 gives

$$
\varphi^{*} \sigma-4 \sigma=I_{*}\left(g^{2} \cdot \sigma\right)+P\left(g, D_{i}, c\right) \quad \text { in } \mathrm{CH}^{2}(F),
$$

for some weighted homogeneous polynomial $P$ of degree $2, D_{i} \in \mathrm{CH}^{1}(F)_{\text {prim }}$. The cycle $g^{2} \cdot \sigma$ can be written as $g^{2} \cdot \sigma=\operatorname{deg}\left(g^{2} \cdot \sigma\right)\left[\mathfrak{o}_{F}\right]+\sigma^{\prime}$, where $\sigma^{\prime} \in \mathrm{CH}^{4}(F)_{\text {hom. }}$. By Lemma A.5, $I_{*} \mathfrak{o}_{F}=\frac{1}{3}\left(g^{2}-c\right)$. Thus $\varphi^{*} \sigma-4 \sigma=I_{*}\left(\sigma^{\prime}\right)+Q\left(g, D_{i}, c\right)$, for some weighted homogeneous polynomial $Q$ of degree 2 . Now $I_{*}\left(\sigma^{\prime}\right)$ belongs to $\mathcal{A}_{\text {hom }}$ so that by Proposition 21.10 we get $\left(\varphi^{*}+2\right) I_{*} \sigma^{\prime}=0$. Recall that $\varphi^{*} c=31 c$ and $\varphi^{*} g^{2}=4 g^{2}+45 c$; see [2, Theorem 8]. Lemma 21.5 gives, when $X$ does not contain any plane, $\varphi^{*} D^{2}=4 D^{2}$ and $\varphi^{*}(g \cdot D)=-14 g \cdot D$ for all $D \in \mathrm{CH}^{1}(F)_{\text {prim. }}$. We can then conclude that $\left(\varphi^{*}+2\right)\left(\varphi^{*}-4\right) \sigma$ is a polynomial in $g, c$ and $D_{i}, D_{i} \in \mathrm{CH}^{1}(F)_{\text {prim }}$. On the other hand, by Proposition 21.7, we know that $\left(\varphi^{*}-31\right)\left(\varphi^{*}+14\right)\left(\varphi^{*}-4\right)$ maps $\mathrm{CH}^{2}(F)$ to $\mathrm{CH}^{2}(F)_{\text {hom }}$. Therefore $\left(\varphi^{*}-31\right)\left(\varphi^{*}+14\right)\left(\varphi^{*}+2\right)\left(\varphi^{*}-4\right) \sigma$ is a polynomial in the variables $g, c$ and $D_{i}$ which is homologically trivial. By the main result of [54], it must be zero modulo rational equivalence. This establishes the polynomial equation of $\varphi^{*}$ on $\mathrm{CH}^{2}(F)$.

Let us now show that $V_{31}^{2}=\langle c\rangle$. First note that for $\sigma \in \mathrm{CH}^{2}(F)_{\text {hom }}$ Proposition 21.3 gives $\varphi^{*} \sigma-4 \sigma=$ $I_{*}\left(g^{2} \cdot \sigma\right)$, and hence $\left(\varphi^{*}+2\right)\left(\varphi^{*}-4\right) \sigma=0$. Consider then $\sigma \in V_{31}^{2}$. By Proposition 21.7, or rather its proof, we see that $[\sigma]$ is proportional to $[c]$. But then, by the above, 31 is not an eigenvalue of $\varphi^{*}$ on $\mathrm{CH}^{2}(F)_{\text {hom }}$. Hence $\sigma$ is proportional to $c$, which proves that $V_{31}^{2}=\langle c\rangle$.

We now show that $V_{-14}^{2}=g \cdot V_{-2}^{1}$. Recall that $V_{-2}^{1}=\mathrm{CH}^{1}(F)_{\text {prim }}$. Lemma 21.5 gives the inclusion $V_{-14}^{2} \supseteq g \cdot V_{-2}^{1}$. Consider then $\sigma \in V_{-14}^{2}$. A quick look at the proof of Proposition 21.7 shows that $[\sigma]$ belongs to $[g] \cdot \mathrm{H}^{2}(F, \mathbb{Q})_{\text {prim. }}$. Since -14 is not an eigenvalue of $\varphi^{*}$ acting on $\mathrm{CH}^{2}(F)_{\text {hom }}$, we get that $\sigma \in g \cdot V_{-2}^{1}$.

Finally let us show that $\mathrm{CH}^{2}(F)_{0}=V_{31}^{2} \oplus V_{-14}^{2} \oplus V_{4}^{2}$. Consider $\sigma \in V_{4}^{2}$. We know that $0=\varphi^{*} \sigma-4 \sigma=$ $I_{*}\left(g^{2} \cdot \sigma-\operatorname{deg}\left(g^{2} \cdot \sigma\right)\left[\mathfrak{o}_{F}\right]\right)+Q\left(g, D_{i}, c\right)$, for some weighted homogeneous polynomial $Q$ of degree 2 . Since $I_{*}\left(g^{2} \cdot \sigma-\operatorname{deg}\left(g^{2} \cdot \sigma\right)\left[\mathfrak{o}_{F}\right]\right)$ belongs to $\mathrm{CH}^{2}(F)_{2}$ and $Q\left(g, D_{i}, c\right)$ belongs to $\mathrm{CH}^{2}(F)_{0}$, we get that $I_{*}\left(g^{2} \cdot \sigma-\operatorname{deg}\left(g^{2} \cdot \sigma\right)\left[\mathfrak{o}_{F}\right]\right)=0$. It immediately follows from (107) and from the identity $I_{*} \mathfrak{o}_{F}=\frac{1}{3}\left(g^{2}-c\right)$ of Lemma A.5 that $L_{*}\left(g^{2} \cdot \sigma\right)=0$. Since by (iii) of Lemma A.3 $c \cdot \sigma$ is a multiple of $\left[\mathfrak{o}_{F}\right]$ for all $\sigma \in \mathrm{CH}^{2}(F)$, we see that $L_{*}(l \cdot \sigma)=0$. Therefore by Theorem $19.2\left(L^{2}\right)_{*} \sigma=2 \sigma$, i.e., $\sigma \in W_{1}^{2} \subset$ 
$\mathrm{CH}^{2}(F)_{0}$. Since $V_{31}^{2} \oplus V_{-14}^{2} \subseteq \mathrm{CH}^{2}(F)_{0}$ and $V_{-2}^{2}=\mathrm{CH}^{2}(F)_{2}$, it is straightforward to conclude that $\mathrm{CH}^{2}(F)_{0}=V_{31}^{2} \oplus V_{-14}^{2} \oplus V_{4}^{2}$.

Now we prove (ii), the decomposition for $\mathrm{CH}^{3}(F)$. First consider $\sigma_{1} \in g \cdot V_{-2}^{2}$. Since $V_{-2}^{2}=\mathcal{A}_{\text {hom }}$ and $I_{*}: V_{-8}^{4} \stackrel{\simeq}{\longrightarrow} \mathcal{A}_{\text {hom }}$ is an isomorphism, we can write $\sigma_{1}=g \cdot I_{*} x$ for some $x \in V_{-8}^{4}$. Then by Proposition 21.3 we have

$$
\varphi^{*} \sigma_{1}=4 \sigma_{1}+3 g \cdot I_{*}\left(g \cdot \sigma_{1}\right)=4 \sigma+3 g \cdot I_{*}\left(g^{2} \cdot I_{*} x\right)=4 \sigma_{1}+3 g \cdot I_{*}(-6 x)=-14 \sigma_{1} .
$$

Here, we used the fact mentioned earlier in the proof that $g^{2} \cdot I_{*}$ acts as multiplication by -6 on $V_{-8}^{4}$. Thus we have shown that $\varphi^{*}=-14$ on $g \cdot V_{-2}^{2}$. The formula for the action of $\varphi^{*}$ on $\mathrm{CH}^{3}(F)_{\text {hom }}$ obtained in Proposition 21.3 shows that for all $\sigma \in \mathrm{CH}^{3}(F)_{\text {hom }}$, we have $\varphi^{*} \sigma-4 \sigma \in g \cdot V_{-2}^{2}$. It follows that $\left(\varphi^{*}+14\right)\left(\varphi^{*}-4\right) \sigma=0$. We may then conclude that $\left(\varphi^{*}-28\right)\left(\varphi^{*}+8\right)\left(\varphi^{*}-4\right)\left(\varphi^{*}+14\right)$ vanishes on $\mathrm{CH}^{3}(F)$ by Proposition 21.7 .

Finally, that $V_{28}^{3}=\left\langle g^{3}\right\rangle, V_{-8}^{3}=g^{2} \cdot V_{-2}^{1}$ and $\mathrm{CH}^{3}(F)_{0}=V_{28}^{3} \oplus V_{-8}^{3}$ follows from the proof of Proposition 21.7 and from the fact proved above that 28 and -8 are not eigenvalues of $\varphi^{*}$ acting on $\mathrm{CH}^{3}(F)_{\text {hom }}$.

Remark 21.11. It is interesting to note the following. Let $F$ be a hyperkähler variety of dimension $2 d$ which is birational to a Lagrangian fibration $\tilde{F} \rightarrow \mathbb{P}^{d}$. Let $B \rightarrow \mathbb{P}^{d}$ be a generically finite map with $B$ smooth projective such that the pull-back $\tilde{F}_{B} \rightarrow B$ admits a section. In that case, $\tilde{F}_{B}$ is an abelian scheme over $B$. Consider then the relative multiplication-by- $n$ map on $\tilde{F}_{B}$. By the work of Deninger-Murre [21], we see that $\mathrm{CH}_{2 d}\left(\tilde{F}_{B}\right)$ splits into eigenspaces for the action of the multiplicationby- $n$ map and that the eigenvalues are $n^{2 d}, n^{2 d-1}, \ldots, n^{d}$. This multiplication-by- $n$ map defines naturally a self-correspondence $\Gamma_{n} \in \mathrm{CH}_{2 d}(F \times F)$ which induces a similar decomposition on $\mathrm{CH}^{2 d}(F)$, i.e., we have $\mathrm{CH}^{2 d}(F)=\mathrm{CH}^{2 d}(F)_{0} \oplus \mathrm{CH}^{2 d}(F)_{2} \oplus \ldots \oplus \mathrm{CH}^{2 d}(F)_{2 d}$ where $\mathrm{CH}^{2 d}(F)_{2 i}=\left\{\sigma \in \mathrm{CH}^{2 d}(F): \Gamma_{n}^{*} \sigma=n^{2 d-i} \sigma\right\}$.

Thus, what we see here, is that the rational map $\varphi: F \rightarrow F$ induces a map $\varphi^{*}: \mathrm{CH}^{4}(F) \rightarrow \mathrm{CH}^{4}(F)$ which splits $\mathrm{CH}^{4}(F)$ in the same way a relative multiplication-by- $(-2)$ map would on a Lagrangian fibration. It would be interesting to show that when $F$ admits a Lagrangian fibration the two splittings are identical.

Let us end this section by the following complement to Theorem 21.9, which deals with the action of $\varphi^{*}$ on $\mathrm{CH}^{2}(F)$ when $X$ may contain some planes.

Lemma 21.12. Let $F$ be the variety of lines on a cubic fourfold $X$. If $\sigma \in \mathrm{CH}^{2}(F)_{0}$, then

$$
\varphi^{*} \sigma=4 \sigma+P\left(g, c, D_{i}\right),
$$

where $P\left(g, c, D_{i}\right), D_{i} \in \mathrm{CH}^{1}(F)$, is a weighted homogeneous polynomial of degree 2 . In particular, if $\sigma \in \mathrm{CH}^{2}(F)_{0, \text { hom }}$, then $\varphi^{*} \sigma=4 \sigma$.

Proof. Let $\sigma$ be a 2-cycle on $F$. By Proposition 21.2, we have

$$
\varphi^{*} \sigma=4 \sigma+I_{*}\left(g^{2} \cdot \sigma\right)+Q\left(D_{i}, g, c, \Pi_{j}^{*}\right)
$$

where $Q\left(D_{i}, g, c, \Pi_{j}^{*}\right)$ is a weighted homogeneous polynomial of degree 2. By Lemma 21.13 below, $\Pi_{j}^{*}$ is a polynomial in $g, c$ and $D_{\Pi_{j}}$, where $D_{\Pi_{j}}$ is the divisor of lines meeting $\Pi_{j}$. Therefore $Q\left(D_{i}, g, c, \Pi_{j}^{*}\right)$ is actually a weighted homogeneous polynomial of degree 2 in the variables $D_{i}, g, c$. Now assume that $\sigma \in \mathrm{CH}^{2}(F)_{0}$. We know from Proposition 4.2 that $l \cdot \sigma$ is a multiple of $\mathfrak{o}_{F}$. Since $g^{2}$ is a linear combination of $l$ and $c$ by (108) and since $c \cdot \tau$ is a multiple of $\mathfrak{o}_{F}$ by Lemma A.3 for all $\tau \in \mathrm{CH}^{2}(F)$, we see that $g^{2} \cdot \sigma$ is a multiple of $\mathfrak{o}_{F}$. Thus, by Lemma A.5, $I_{*}\left(g^{2} \cdot \sigma\right)$ is a linear combination of $g^{2}$ and $c$. All in all, this yields the first part of the lemma.

Now, if $\sigma$ is homologically trivial, then $P\left(g, c, D_{i}\right)$ is homologically trivial. By [54], it follows that $P\left(g, c, D_{i}\right)=0$.

Lemma 21.13. Let $\Pi \subset X$ be a plane and $\Pi^{*} \subset F$ the dual plane. Then the class of $\Pi^{*}$ in $\mathrm{CH}^{2}(F)$ is a linear combination of $g^{2}, c,\left(D_{\Pi}\right)^{2}$ and $g \cdot D_{\Pi}$, where $D_{\Pi}$ is the divisor of all lines meeting $\Pi$. 
Proof. This is implicitly contained in $\left[54\right.$, p.646] and we repeat the proof for completeness. Let $\widetilde{D}=q^{-1} \Pi$ then $\widetilde{D}$ is isomorphic to $D=D_{\Pi}$ away from $\Pi^{*}$. Thus under this identification, we get

$$
\left.D\right|_{D}=\operatorname{det}\left(q^{*} \mathscr{N}_{\Pi / X}\right)-\left.\mathscr{T}_{P / F}\right|_{\widetilde{D}}=0-\left(2 q^{*} h-p^{*} g\right)=p^{*} g-2 q^{*} h,
$$

away from $\Pi^{*}$. By pushing forward to $F$ and noting that the class $\left.h\right|_{\Pi}$ is represented by a line on $\Pi$, we get

$$
\left(D_{\Pi}\right)^{2}=a \Pi^{*}+g \cdot D_{\Pi}-2 S_{l}
$$

for some integer $a \in \mathbb{Z}$, where $l \subset \Pi$ is a line. Note that $[l]=\mathfrak{o}_{F}$ (see Lemma A.3) and $S_{l}=I_{*}[l]=$ $I_{*} \mathfrak{o}_{F}=\frac{1}{3}\left(g^{2}-c\right)$ (see Lemma A.5). To conclude, we still have to show that $a \neq 0$. But this is clear since if $a=0$ then $c$ would be a linear combination of intersections of divisors and we know this is not the case. Hence $a \neq 0$ and the Lemma follows.

\subsection{Compatibility of the action of $\varphi$ with the Fourier transform.}

Proposition 21.14. The rational map $\varphi: F \rightarrow F$ preserves the Fourier decomposition, i.e., $\varphi^{*}$ maps $\mathrm{CH}^{i}(F)_{s}$ into $\mathrm{CH}^{i}(F)_{s}$ for all $i$ and all $s$.

Proof. By Theorem 21.9, the only thing to check is that $\varphi^{*} \sigma \in \mathrm{CH}^{2}(F)_{0}$ for all $\sigma \in \mathrm{CH}^{2}(F)_{0}$. But this is a consequence of Lemma 21.12, together with Theorem 4.6 which shows that any weighted homogeneous polynomial $P\left(g, c, D_{i}\right)$ of degree 2 belongs to $\mathrm{CH}^{2}(F)_{0}$ (recall that $c$ is a linear combination of $l$ and $\left.g^{2}\right)$.

Actually we have the following more precise result which implies that $\varphi^{*}$ commutes with $\mathcal{F} \circ \mathcal{F}$ on $\mathrm{CH}^{i}(F)_{s}$ as long as $(i, s) \neq(2,0)$. On $\mathrm{CH}^{2}(F)_{0}$, it is not true that $\varphi_{*} \mathcal{F}=\mathcal{F} \varphi^{*}$ because both $\varphi_{*}=\varphi^{*}$ and $\mathcal{F}$ are diagonalizable but $\langle l\rangle$ is fixed by $\mathcal{F}$ and $\varphi^{*} l=\varphi_{*} l \notin\langle l\rangle$.

Proposition 21.15. Let $F$ be the variety of lines on a cubic fourfold $X$. Then

$$
\begin{aligned}
\varphi_{*} \mathcal{F} & =\mathcal{F} \varphi^{*}: \mathrm{CH}^{i}(F)_{s} \rightarrow \mathrm{CH}^{4-i+s}(F)_{s} \quad \text { for all }(i, s) \neq(2,0) ; \\
\varphi_{*} & =\varphi^{*}: \mathrm{CH}^{2}(F)_{0} \rightarrow \mathrm{CH}^{2}(F)_{0} .
\end{aligned}
$$

Proof. By Theorem 2, $\mathcal{F}$ induces isomorphisms $\mathrm{CH}^{i}(F)_{s} \rightarrow \mathrm{CH}^{4-i+s}(F)_{s}$. By Theorem 21.9, the action of $\varphi^{*}$ is multiplication by $(-2)^{4-s}$ on $\mathrm{CH}^{4}(F)_{2 s}$ and is multiplication by -2 on $\mathrm{CH}^{2}(F)_{2}$. By Proposition B.7, we know that $\varphi_{*} \varphi^{*}$ is multiplication by 16 on $\mathrm{CH}^{4}(F)$ and on $\mathrm{CH}^{2}(F)_{\text {hom }}$. Therefore the proposition is settled for $i=0$ or 4 and $(i, s)=(2,2)$. For $i=3$, we have $\mathrm{CH}^{3}(F)_{2}=\mathrm{CH}^{3}(F)_{\text {hom }}$ and $\mathcal{F}$ acts on $\mathrm{CH}^{3}(F)_{2}$ as the identity. We can then conclude in the case $(i, s)=(3,2)$ from Proposition 21.3 where it is shown that $\varphi_{*}$ and $\varphi^{*}$ have the same action on $\mathrm{CH}^{3}(F)_{\text {hom }}$. Let us now consider $(i, s)=(3,0)$ or $(1,0)$. Let $D$ be a divisor on $F$. One can check from the proof of Theorem 2.2 that $\mathcal{F}(D)=\frac{1}{2} l \cdot D$ and that $\mathcal{F}(l \cdot D)=25 D$. If $D \in \mathrm{CH}^{1}(F)_{\text {prim }}$, then by [54, (3.36)] $c \cdot D=0$ so that $l \cdot D$ is proportional to $g^{2} \cdot D$; if $D \in\langle g\rangle$, then by [54, Lemma 3.5] $c \cdot D$ is proportional to $g^{3}$ so that $l \cdot D$ is proportional to $g^{3}$. In the proof of Proposition 21.7, we determined the action of $\varphi^{*}$ on $\mathrm{H}^{2}(F, \mathbb{Q})$ and on $g^{2} \cdot \mathrm{H}^{2}(F, \mathbb{Q})$. Since both $\mathrm{CH}^{3}(F)_{0}$ and $\mathrm{CH}^{1}(F)_{0}$ inject into cohomology via the cycle class map, we need only to determine the action of $\varphi_{*}$ on $\mathrm{H}^{2}(F, \mathbb{Q})$ and on $g^{2} \cdot \mathrm{H}^{2}(F, \mathbb{Q})$. On the one hand, we have $\varphi_{*} g=28 g$ and $\varphi_{*} \omega=-8 \omega$ because $-2 \tau^{*} \omega=\widetilde{\varphi}^{*} \omega$. On the other hand, we have $\varphi_{*} g^{3}=7 g$ and $\varphi_{*}\left(\left[g^{2}\right] \cup \omega\right)=-\frac{1}{2} \widetilde{\varphi}_{*}\left(\tau^{*}\left[g^{2}\right] \cup \widetilde{\varphi}^{*} \omega\right)=$ $-\frac{1}{2} \varphi_{*}\left[g^{2}\right] \cup \omega=-2\left[g^{2}\right] \cup \omega$. This proves that $\varphi_{*} \mathcal{F}=\mathcal{F} \varphi^{*}$ on $\mathrm{CH}^{3}(F)_{0}$ and on $\mathrm{CH}^{1}(F)_{0}$.

It remains to see that $\varphi_{*}=\varphi^{*}$ on $\mathrm{CH}^{2}(F)_{0}$. Consider $\sigma \in \mathrm{CH}^{2}(F)_{0}$. By Lemma 21.12, we know that $\varphi^{*} \sigma=4 \sigma+P\left(g, c, D_{i}\right)$, where $P\left(g, c, D_{i}\right), D_{i} \in \mathrm{CH}^{1}(F)$, is a weighted homogeneous polynomial of degree 2. The exact same arguments as in the proof of Lemma 21.12 show that likewise we have $\varphi_{*} \sigma=4 \sigma+Q\left(g, c, D_{i}\right)$, where $Q\left(g, c, D_{i}\right), D_{i} \in \mathrm{CH}^{1}(F)$, is a weighted homogeneous polynomial of degree 2. Thus

$$
\varphi^{*} \sigma-\varphi_{*} \sigma=(P-Q)\left(g, c, D_{i}\right) .
$$

By Theorem 4.6, it is then enough to check that $\varphi_{*}=\varphi^{*}$ on $\mathrm{H}^{4}(F, \mathbb{Q})$. We know from Proposition 21.4 that this is the case on $\left\langle g^{2}, c\right\rangle$. From the proof of Proposition 21.7, it is enough to check that $\varphi_{*}([g] \cup \omega)=-14[g] \cup \omega$ and $\varphi_{*}\left(\omega^{2}\right)=4 \omega^{2}$. But then this follows immediately from $-2 \tau^{*} \omega=\widetilde{\varphi}^{*} \omega$ and from $\varphi_{*} g=28 g$. 


\section{The Fourier Decomposition for $F$ is multiplicative}

In this section, we use the rational map $\varphi: F \rightarrow F$ to investigate the compatibility of the Fourier decomposition with the intersection product on $\mathrm{CH}^{*}(F)$. The concatenation of all the results of this section provides a proof of Theorem 3 for $F$ the variety of lines on a very general cubic fourfold.

22.1. Intersection with homologically trivial 2-cycles. Because $\varphi$ is only a rational map, $\varphi^{*}$ does not respect the intersection product in general. Yet we have the following observation.

Lemma 22.1. If $\alpha \in \mathrm{CH}^{2}(F)_{\text {hom }}$, then $\varphi^{*} \alpha \cdot \varphi^{*} \beta=\varphi^{*}(\alpha \cdot \beta)$ for all $\beta \in \mathrm{CH}^{*}(F)$.

Proof. This is Proposition B.6 in view of the fact that $\mathrm{CH}^{2}(F)_{\text {hom }}=T^{2}(F)$, where $T^{2}(F)$ is the kernel of the Abel-Jacobi map $\mathrm{CH}^{2}(F)_{\text {hom }} \rightarrow J^{2}(F)$, because $J^{2}(F)$ is a sub-quotient of $\mathrm{H}^{3}(F, \mathbb{C})=0$.

Proposition 22.2. Let $F$ be the variety of lines on a cubic fourfold $X$. Then the Fourier decomposition satisfies

Moreover, we have

$$
\mathrm{CH}^{4}(F)_{2}=\mathrm{CH}^{1}(F)_{0}^{\cdot 2} \cdot \mathrm{CH}^{2}(F)_{2} .
$$

$$
g \cdot D \cdot \mathrm{CH}^{2}(F)_{2}=0, \quad \text { for all } D \in \mathrm{CH}^{1}(F)_{\text {prim }} .
$$

Proof. Recall that $\mathrm{CH}^{2}(F)_{2}=V_{-2}^{2}$. By Lemma 21.5, we have for all $D \in \mathrm{CH}^{1}(F)_{\text {prim }}$

$$
\begin{aligned}
\varphi^{*}(g \cdot D) & =\tau_{*} \widetilde{\varphi}^{*}(g \cdot D)=\tau_{*}\left(\left(7 \tau^{*} g-3 E+\sum a_{g, i} E_{i}\right) \cdot\left(-2 \tau^{*} D+\sum a_{D, i} E_{i}\right)\right) \\
& =-14 g \cdot D+\sum b_{g, D, i} \Pi_{i}^{*},
\end{aligned}
$$

where the $b_{g, D, i}$ are rational numbers and the $\Pi_{i}^{*}$ are the dual planes to the planes contained in $X$. Likewise, we have $\varphi^{*} g^{2}=4 g^{2}+45 c+\sum b_{g^{2}, i} \Pi_{i}^{*}$ and $\varphi^{*} D^{2}=4 D^{2}+\sum b_{D^{2}, i} \Pi_{i}^{*}$ for all $D \in \mathrm{CH}^{1}(F)_{\text {prim }}$.

Since for all $\sigma \in \mathrm{CH}^{2}(F)_{\text {hom }} c \cdot \sigma=0$ by Lemma A.3(iii) and $\Pi_{i}^{*} \cdot \sigma=0$, we see thanks to Lemma 22.1 that for all $D \in \mathrm{CH}^{1}(F)_{\text {prim }}$ and for all $\sigma \in \mathrm{CH}^{2}(F)_{2}$ we have

$$
\begin{gathered}
\varphi^{*}\left(g^{2} \cdot \sigma\right)=-8 g^{2} \cdot \sigma, \\
\varphi^{*}\left(D^{2} \cdot \sigma\right)=-8 D^{2} \cdot \sigma, \\
\varphi^{*}(g \cdot D \cdot \sigma)=28 g \cdot D \cdot \sigma .
\end{gathered}
$$

The proposition then follows because 28 is not an eigenvalue of $\varphi^{*}$ acting on $\mathrm{CH}^{4}(F)$ by Theorem 21.9 and because $g^{2} \cdot: \mathrm{CH}^{2}(F)_{2} \rightarrow \mathrm{CH}^{4}(F)_{2}$ is an isomorphism.

Since $\mathrm{CH}^{1}(F)_{0}^{2} \subseteq \mathrm{CH}^{2}(F)_{0}$ by Theorem 4.6, the following is a refinement of the first statement of Proposition 22.2.

Proposition 22.3. Let $F$ be the variety of lines on a cubic fourfold. Then

$$
\mathrm{CH}^{2}(F)_{0} \cdot \mathrm{CH}^{2}(F)_{2}=\mathrm{CH}^{4}(F)_{2} .
$$

Proof. By Proposition 21.10, we know that $\mathrm{CH}^{2}(F)_{2}=V_{-2}^{2}$ consists of homologically trivial cycles. Consider $\sigma \in \mathrm{CH}^{2}(F)_{0}$ and $\tau \in \mathrm{CH}^{2}(F)_{2}$. By Lemmas 22.1 and 21.12, we have

$$
\varphi^{*}(\sigma \cdot \tau)=\varphi^{*} \sigma \cdot \varphi^{*} \tau=\left(4 \sigma+P\left(g, c, D_{i}\right)\right) \cdot(-2 \tau)=-8 \sigma \cdot \tau-2 P\left(g, c, D_{i}\right) \cdot \tau .
$$

Since $c \cdot \tau=0$, by Proposition 22.2 we obtain that $P\left(g, c, D_{i}\right) \cdot \tau$ belongs to $\mathrm{CH}^{4}(F)_{2}$. It then immediately follows from the fact that $\mathrm{CH}^{4}(F)_{2}=V_{-8}^{4}$ that $\sigma \cdot \tau \in \mathrm{CH}^{4}(F)_{2}$.

Proposition 22.4. Let $F$ be the variety of lines on a cubic fourfold. Then

$$
\mathrm{CH}^{1}(F) \cdot \mathrm{CH}^{2}(F)_{0, \text { hom }}=0 \text {. }
$$

Proof. Indeed, if $\sigma \in \mathrm{CH}^{2}(F)_{0, \text { hom }}$, then by Lemma $21.12 \varphi^{*} \sigma=4 \sigma$. It follows from Lemma 22.1 that $\varphi^{*}(D \cdot \sigma)=4 \sigma \cdot \varphi^{*} D$ for all $D \in \mathrm{CH}^{1}(F)$. Depending whether $D \in\langle g\rangle$ or $D \in \mathrm{CH}^{1}(F)_{\text {prim }}$, we see that $\varphi^{*}(D \cdot \sigma)=28 \sigma \cdot D$ or $-8 \sigma \cdot D$. By Theorem 21.9, neither 28 nor -8 are eigenvalues of $\varphi^{*}$ acting on $\mathrm{CH}^{3}(F)_{\text {hom }}=\mathrm{CH}^{3}(F)_{2}$. Hence $D \cdot \sigma=0$.

Proposition 22.5. Let $F$ be the variety of lines on a cubic fourfold. Then 


$$
\mathrm{CH}^{2}(F)_{0} \cdot \mathrm{CH}^{2}(F)_{0, \text { hom }}=0 .
$$

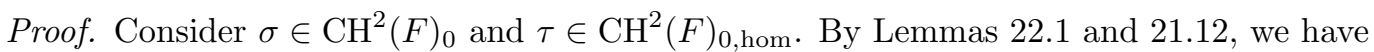

$$
\varphi^{*}(\sigma \cdot \tau)=\varphi^{*} \sigma \cdot \varphi^{*} \tau=\left(4 \sigma+P\left(g, c, D_{i}\right)\right) \cdot 4 \tau=16 \sigma \cdot \tau+4 P\left(g, c, D_{i}\right) \cdot \tau .
$$

But then, as in the proof of Proposition 22.2, we see that $\varphi^{*}\left(g^{2} \cdot \tau\right)=16 g^{2} \cdot \tau, \varphi^{*}\left(D^{2} \cdot \tau\right)=16 D^{2} \cdot \tau$ and $\varphi^{*}(g \cdot D \cdot \tau)=-14 \cdot 4 g \cdot D \cdot \tau$ for all $D \in \mathrm{CH}^{1}(F)_{\text {prim }}$. Also, we have $\varphi^{*}(c \cdot \tau)=31 \cdot 4 c \cdot \tau$. Since none of these numbers are eigenvalues of $\varphi^{*}$ acting on $\mathrm{CH}^{4}(F)_{\text {hom }}$, we see that $P\left(g, c, D_{i}\right) \cdot \tau=0$. Thus $\varphi^{*}(\sigma \cdot \tau)=16 \sigma \cdot \tau$. Again, 16 is not an eigenvalue of $\varphi^{*}$ acting on $\mathrm{CH}^{4}(F)_{\text {hom }}$ and hence $\sigma \cdot \tau=0$.

22.2. Intersection with divisors. We know that $\mathrm{CH}^{3}(F)_{2}=\mathrm{CH}^{3}(F)_{\text {hom }}$ and $\mathrm{CH}^{2}(F)_{2} \subseteq \mathrm{CH}^{2}(F)_{\text {hom }}$, so that it is clear that $\mathrm{CH}^{1}(F) \cdot \mathrm{CH}^{2}(F)_{2} \subseteq \mathrm{CH}^{3}(F)_{2}$. The following is thus a strengthening of the equality $\mathrm{CH}^{4}(F)_{2}=\mathrm{CH}^{1}(F)^{\cdot 2} \cdot \mathrm{CH}^{2}(F)_{2}$ of Proposition 22.2.

Proposition 22.6. Let $F$ be the variety of lines on a cubic fourfold $X$. Then

$$
\mathrm{CH}^{1}(F) \cdot \mathrm{CH}^{3}(F)_{2}=\mathrm{CH}^{4}(F)_{2} \text {. }
$$

Proof. Recall from Theorems $2.2 \& 2.4$ that $\mathrm{CH}^{3}(F)_{\text {hom }}=\mathrm{CH}^{3}(F)_{2}$. Let us first compute $\varphi^{*}(g \cdot \sigma)$ for $\sigma \in \mathrm{CH}^{3}(F)_{2}$. By Lemma 21.5, we have $\widetilde{\varphi}^{*} g=7 \tau^{*} g-3 E+\sum a_{g, i} E_{i}$ for some numbers $a_{g, i} \in \mathbb{Z}$. Thus

$$
\varphi^{*}(g \cdot \sigma)=\tau_{*}\left(\left(7 \tau^{*} g-3 E+\sum a_{g, i} E_{i}\right) \cdot \widetilde{\varphi}^{*} \sigma\right)=7 g \cdot \varphi^{*} \sigma-3 \tau_{*}\left(E \cdot \widetilde{\varphi}^{*} \sigma\right)+\sum a_{g, i} \tau_{*}\left(E_{i} \cdot \widetilde{\varphi}^{*} \sigma\right) .
$$

Now $\widetilde{\varphi}^{*} \sigma$ is a homologically trivial cycles so that $\tau_{*}\left(E \cdot \widetilde{\varphi}^{*} \sigma\right)$ is a homologically trivial zero-cycle supported on $\Sigma_{2}$. By Proposition 19.5 we get $\tau_{*}\left(E \cdot \widetilde{\varphi}^{*} \sigma\right) \in \mathrm{CH}^{4}(F)_{2}=V_{-8}^{4}$. Likewise $\tau_{*}\left(E_{i} \cdot \widetilde{\varphi}^{*} \sigma\right)$ is a homologically trivial cycle support on the plane $\Pi_{i}^{*}$ and is therefore zero. By Theorem 21.9, the action of $\varphi^{*}$ on $\mathrm{CH}^{3}(F)_{\text {hom }}$ diagonalizes with eigenvalues 4 and -14 . Since $7 \cdot 4$ and $7 \cdot(-14)$ are not eigenvalues of $\varphi^{*}$ acting on $\mathrm{CH}^{4}(F)$ we conclude that $g \cdot \sigma \in \mathrm{CH}^{4}(F)_{2}=V_{-8}^{4}$.

Consider now $D \in V_{-2}^{1}=\mathrm{CH}^{1}(F)_{\text {prim }}$. In that case, we have $\widetilde{\varphi}^{*} D=-2 \tau^{*} D+\sum a_{D, i} E_{i}$ for some numbers $a_{D, i} \in \mathbb{Z}$. Thus

$$
\varphi^{*}(D \cdot \sigma)=\tau_{*}\left(\left(-2 \tau^{*} D+\sum a_{D, i} E_{i}\right) \cdot \widetilde{\varphi}^{*} \sigma\right)=-2 D \cdot \varphi^{*} \sigma+\sum a_{D, i} \tau_{*}\left(E_{i} \cdot \widetilde{\varphi}^{*} \sigma\right)=-2 D \cdot \varphi^{*} \sigma .
$$

It follows that $\varphi^{*}(D \cdot \sigma) \in V_{-8}^{4}$ if $\varphi^{*} \sigma=4 \sigma$ and that $\varphi^{*}(D \cdot \sigma)=0$ if $\varphi^{*} \sigma=-14 \sigma$.

Proposition 22.7. Let $F$ be the variety of lines on a cubic fourfold $X$. Then

$$
\mathrm{CH}^{1}(F)_{\text {prim }} \cdot \mathrm{CH}^{2}(F)_{0}=\mathrm{CH}^{3}(F)_{0} .
$$

Moreover, if $X$ is very general, then $\mathrm{CH}^{1}(F) \cdot \mathrm{CH}^{2}(F)_{0}=\mathrm{CH}^{3}(F)_{0}$.

Proof. Let us consider $D \in \mathrm{CH}^{1}(F)_{\text {prim }}$ and $\sigma \in \mathrm{CH}^{2}(F)_{2}$. We have

$$
\begin{aligned}
\varphi^{*}(\sigma \cdot D) & =\tau_{*}\left(\widetilde{\varphi}^{*} \sigma \cdot \widetilde{\varphi}^{*} D\right) \\
& =\tau_{*}\left(\left(4 \tau^{*} \sigma+\tau^{*} P\left(g, c, D_{i}\right)+\alpha\right) \cdot\left(-2 \tau^{*} D+\sum a_{D, i} E_{i}\right)\right) \\
& =-8 \sigma \cdot D-2 P\left(g, c, D_{i}\right) \cdot D+\sum a_{D, i} \tau_{*}\left(\alpha \cdot E_{i}\right) .
\end{aligned}
$$

For the second equality, we have used Lemmas 21.5 and 21.12, and $\alpha$ is a 2-cycle on $\widetilde{F}$ such that $\tau_{*} \alpha=0$. The third equality is the projection formula coupled with $\tau_{*} E_{i}=0$. Since $l$ is a linear combination of $g^{2}$ and $c$, Theorem 4.6 gives $P\left(g, c, D_{i}\right) \cdot D \in \mathrm{CH}^{3}(F)_{0}$. On the other hand, $\tau_{*}\left(\alpha \cdot E_{i}\right)$ is a 1-cycle supported on $\Pi_{i}^{*}$. Therefore, $\tau_{*}\left(\alpha \cdot E_{i}\right)$ is rationally equivalent to a multiple of a rational curve on $F$ that contains a point rationally equivalent to $\mathfrak{o}_{F}$ (for instance because $c \cdot \Pi_{i}^{*}$ is a multiple of $\mathfrak{o}_{F}$ ). Now we claim that the class of every rational curve $C$ on $F$ that contains a point rationally equivalent to $\mathfrak{o}_{F}$ is in $\mathrm{CH}^{3}(F)_{0}$. Let us finish the proof assuming the claim. First, we see that

$$
\varphi^{*}(\sigma \cdot D)+8 \sigma \cdot D \in \mathrm{CH}^{3}(F)_{0} .
$$

Second, we know from Theorem 21.9 that $\mathrm{CH}^{3}(F)_{0}=V_{28}^{3} \oplus V_{-8}^{3}$ and that $\mathrm{CH}^{3}(F)_{2}=V_{4}^{3} \oplus V_{-14}^{3}$. We immediately get that $\sigma \cdot D \in \mathrm{CH}^{3}(F)_{0}$.

Let us now prove the claim. As in the proof of [54, Corollary 3.4], note that $I^{2}$ is the restriction of $I \times I$ to the diagonal $\Delta_{F \times F}$ of $(F \times F)^{2}$. Therefore $\left(I^{2}\right)_{*} C=\left.\left((I \times I)_{*}\left(\iota_{\Delta}\right)_{*} C\right)\right|_{\Delta_{F \times F}}$. But then, 
MINGMIN SHEN AND CHARLES VIAL

because $C$ is a rational curve and because it contains a point rationally equivalent to $\mathfrak{o}_{F}$, we have $\left(\iota_{\Delta}\right)_{*} C=\mathfrak{o}_{F} \times C+C \times \mathfrak{o}_{F}$. This immediately yields $\left(I^{2}\right)_{*} C=2 I_{*} C \cdot I_{*} \mathfrak{o}_{F}$. Since $I_{*} \mathfrak{o}_{F}$ is a linear combination of $l$ and $g^{2}$, Theorem 4.6 shows that $\left(I^{2}\right)_{*} C \in \mathrm{CH}^{3}(F)_{0}$. By comparing (106) and (109), one may see that, for $\mu \in \mathrm{CH}^{3}(F)_{2}=\mathrm{CH}^{3}(F)_{\text {hom }}, \varphi^{*} \mu=4 \mu$ if and only if $\left(I^{2}\right)_{*} \mu=2 \mu$ and that $\varphi^{*} \mu=-14 \mu$ if and only if $\left(I^{2}\right)_{*} \mu=-4 \mu$. Thus, in view of Theorem $21.9, I^{2}$ induces an automorphism of $\mathrm{CH}^{3}(F)_{\text {hom }}$ and we obtain that $C \in \mathrm{CH}^{3}(F)_{0}$.

Finally, if $X$ is very general, then (115) shows that $\mathrm{CH}^{2}(F)_{0}$ is spanned by $l, g^{2}$ and by $\mathrm{CH}^{2}(F)_{0, \text { hom }}$. The proposition is thus a combination of Proposition 22.4, Theorem 4.6 and Proposition 4.2.

22.3. Self-intersection of 2-cycles. We already proved that $\mathrm{CH}^{2}(F)_{2} \cdot \mathrm{CH}^{2}(F)=\mathrm{CH}^{4}(F)_{4}$ and that $\mathrm{CH}^{2}(F)_{0} \cdot \mathrm{CH}^{2}(F)_{2}=\mathrm{CH}^{4}(F)_{2}$. We cannot prove that $\mathrm{CH}^{2}(F)_{0} \cdot \mathrm{CH}^{2}(F)_{0}=\mathrm{CH}^{4}(F)_{0}$ in general but we have the following.

Proposition 22.8. Let $F$ be the variety of lines on a cubic fourfold $X$. Then

$$
\mathrm{CH}^{2}(F)_{0} \cdot \mathrm{CH}^{2}(F)_{0} \subseteq \mathrm{CH}^{4}(F)_{0} \oplus \mathrm{CH}^{4}(F)_{2} .
$$

If $X$ is a very general cubic, then $\mathrm{CH}^{2}(F)_{0} \cdot \mathrm{CH}^{2}(F)_{0}=\mathrm{CH}^{4}(F)_{0}$.

Proof. First we show that

$$
\mathrm{CH}^{1}(F) \cdot \mathrm{CH}^{1}(F) \cdot \mathrm{CH}^{2}(F)_{0} \subseteq \mathrm{CH}^{4}(F)_{0} \oplus \mathrm{CH}^{4}(F)_{2} \cdot
$$

Indeed, if $D, D^{\prime} \in \mathrm{CH}^{1}(F)$ and $\sigma \in \mathrm{CH}^{2}(F)_{0}$, then $D^{\prime} \cdot \sigma \in \mathrm{CH}^{3}(F)=\mathrm{CH}^{3}(F)_{0} \oplus \mathrm{CH}^{3}(F)_{2}$, so that by Theorem 4.6 and Proposition 22.6 we get $D \cdot D^{\prime} \cdot \sigma \in \mathrm{CH}^{4}(F)_{0} \oplus \mathrm{CH}^{4}(F)_{2}$.

Let us now consider $\sigma$ and $\sigma^{\prime} \in \mathrm{CH}^{2}(F)_{0}$. By Lemma 21.12, we have

$$
\widetilde{\varphi}^{*} \sigma=4 \tau^{*} \sigma+\tau^{*} P+\alpha \quad \text { and } \quad \widetilde{\varphi}^{*} \sigma^{\prime}=4 \tau^{*} \sigma^{\prime}+\tau^{*} P^{\prime}+\alpha^{\prime},
$$

where $P, P^{\prime}$ are weighted homogeneous polynomials of degree 2 in $g, c, D_{i}$, and $\alpha, \alpha^{\prime}$ are cycles supported on $E$ such that $\tau_{*} \alpha=0$ and $\tau_{*} \alpha^{\prime}=0$. We then have

$$
\begin{aligned}
\varphi^{*}\left(\sigma \cdot \sigma^{\prime}\right) & =\tau_{*}\left(\widetilde{\varphi}^{*} \sigma \cdot \widetilde{\varphi}^{*} \sigma^{\prime}\right) \\
& =16 \sigma \cdot \sigma^{\prime}+\sigma^{\prime} \cdot P+\sigma \cdot P^{\prime}+P \cdot P^{\prime}+\tau_{*}\left(\alpha \cdot \alpha^{\prime}\right) .
\end{aligned}
$$

By Theorem 4.6, $P \cdot P^{\prime} \in \mathrm{CH}^{4}(F)_{0}$. By (118), $\sigma^{\prime} \cdot P$ and $\sigma \cdot P^{\prime}$ belong to $\mathrm{CH}^{4}(F)_{0} \oplus \mathrm{CH}^{4}(F)_{2}$. The cycle $\tau_{*}\left(\alpha \cdot \alpha^{\prime}\right)$ is supported on $\Sigma_{2}$ so that, by Proposition $19.5, \tau_{*}\left(\alpha \cdot \alpha^{\prime}\right) \in \mathrm{CH}^{4}(F)_{0} \oplus \mathrm{CH}^{4}(F)_{2}$. It is then immediate to conclude from Theorem 21.9 that $\sigma \cdot \sigma^{\prime} \in \mathrm{CH}^{4}(F)_{0} \oplus \mathrm{CH}^{4}(F)_{2}$.

If $X$ is very general, then (115) shows that $\mathrm{CH}^{2}(F)_{0}$ is spanned by $l$, by $g^{2}$ and by $\mathrm{CH}^{2}(F)_{0, \text { hom }}$. That $\mathrm{CH}^{2}(F)_{0} \cdot \mathrm{CH}^{2}(F)_{0}=\mathrm{CH}^{4}(F)_{0}$ is thus a combination of Proposition 22.5, Theorem 4.6 and Proposition 4.2 .

Remark 22.9 (About the intersection with $\left.\mathrm{CH}^{2}(F)_{0}\right)$. The results of this section prove Theorem 3 for the variety of lines on a very general cubic fourfold. In order to prove Theorem 3 for the variety of lines on a (not necessarily very general) cubic fourfold, one would need to prove that $g \cdot \mathrm{CH}^{2}(F)_{0}=\mathrm{CH}^{3}(F)_{0}$ and that $\mathrm{CH}^{2}(F)_{0} \cdot \mathrm{CH}^{2}(F)_{0}=\mathrm{CH}^{4}(F)_{0}$.

That $g \cdot \mathrm{CH}^{2}(F)_{0}=\mathrm{CH}^{3}(F)_{0}$ would follow from knowing that the image of $\mathrm{CH}^{1}\left(\Sigma_{2}\right) \rightarrow \mathrm{CH}^{3}(F)$ is contained in $\mathrm{CH}^{3}(F)_{0}$. In addition, that $\mathrm{CH}^{2}(F)_{0} \cdot \mathrm{CH}^{2}(F)_{0}=\mathrm{CH}^{4}(F)_{0}$ would follow from knowing that the image of $\mathrm{CH}^{1}\left(\Sigma_{2}\right) \cdot \mathrm{CH}^{1}\left(\Sigma_{2}\right) \rightarrow \mathrm{CH}^{4}(F)$ is contained in $\mathrm{CH}^{4}(F)_{0}$.

\section{Appendix A. Some geOmetry of CubiC Fourfolds}

In this appendix we review and prove some results on the geometry of a smooth cubic fourfold and its variety of lines. We fix an algebraically closed field $k$ of characteristic different from 2 or 3 . Let $X \subset \mathbb{P}(V)$ be a smooth cubic fourfold, where $V$ is a 6 -dimensional vector space over $k$. Let $F$ be the variety of lines on $X$, which is smooth of dimension 4 by [5]. When $k=\mathbb{C}$, it is also known that $F$ is a hyperkähler manifold of $\mathrm{K}^{[2]}$-type ; see [10]. Let $h \in \mathrm{Pic}(X)$ be the class of a hyperplane section. The variety $F$ is naturally a sub-variety of $G(2, V)$ and hence inherits a natural rank 2 sub-bundle $\mathscr{E}_{2} \subset V$. Let $g=-c_{1}\left(\mathscr{E}_{2}\right)$ be the Plücker polarization and $c=c_{2}\left(\mathscr{E}_{2}\right)$. 
A.1. Some natural cycle classes. As a sub-variety of $G(2, V)$, the variety $F$ can be viewed as the zero locus of a section of $\mathrm{Sym}^{3} \mathscr{E}_{2}^{\vee}$; see [5]. This description also gives a short exact sequence

$$
\left.\left.0 \longrightarrow \mathscr{T}_{F} \longrightarrow \mathscr{T}_{G(2, V)}\right|_{F} \longrightarrow \operatorname{Sym}^{3} \mathscr{E}_{2}\right|_{F} \longrightarrow 0
$$

Then a routine Chern class computation gives the following

Lemma A.1. $c_{2}(F)=5 g^{2}-8 c$.

Over $F$, we have the universal family of lines

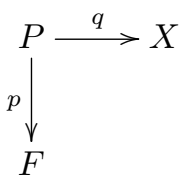

Definition A.2. Associated with the above situation, we define the cylinder homomorphism as $\Psi=$ $q_{*} p^{*}: \mathrm{CH}^{i}(F) \rightarrow \mathrm{CH}^{i-1}(X)$ and the Abel-Jacobi homomorphism as $\Phi=p_{*} q^{*}: \mathrm{CH}^{i}(X) \rightarrow \mathrm{CH}^{i-1}(F)$.

It is proved in [54] that $F$ contains a surface $W$ which represents $c=c_{2}\left(\mathscr{E}_{2}\right)$ in $\mathrm{CH}^{2}(F)$. Furthermore, any two points on $W$ are rationally equivalent on $F$. Hence a point on $W$ defines a special degree 1 element $\mathfrak{o}_{F} \in \mathrm{CH}_{0}(F)$. We summarize some results we need.

Lemma A.3. The following statements hold true.

(i) Any weighted homogeneous polynomial of degree 4 in $g$ and $c$ is a multiple of $\mathfrak{o}_{F}$ in $\mathrm{CH}_{0}(F)$. To be more precise, we have $g^{4}=108 \mathfrak{o}_{F}, c^{2}=27 \mathfrak{o}_{F}$ and $g^{2} c=45 \mathfrak{o}_{F}$.

(ii) Let $C_{x} \subset F$ be the curve of all lines passing through a general point $x \in X$, then $g \cdot C_{x}=6 \mathfrak{o}_{F}$ in $\mathrm{CH}_{0}(F)$.

(iii) For any 2-cycle $\gamma \in \mathrm{CH}^{2}(F)$, the intersection $\gamma \cdot c$ is a multiple of $\mathfrak{o}_{F}$ in $\mathrm{CH}_{0}(F)$.

(iv) If $\Pi=\mathbb{P}^{2} \subset X$ is a plane contained in $X$, then $[l]=\mathfrak{o}_{F}$ for any line $l \subset \Pi$.

(v) Let $l \subset X$ be a line such that $[l]=\mathfrak{o}_{F}$, then $3 l=h^{3}$ in $\mathrm{CH}^{3}(X)$.

Proof. For (i), (ii) and (iii), we refer to [54]. For (iv), we take $\gamma$ to be the lines on $\Pi$ and then apply (iii). To prove $(v)$, we note that the class $c^{2}$ can be represented by the sum of all points corresponding to lines on the intersection $Y=H_{1} \cap H_{2}$ of two general hyperplanes on $X$. Since $Y$ is a smooth cubic surface, it contains exactly 27 lines. These 27 lines can be divided into 9 groups, where each group forms a triangle ; see Definition 20.4. Statement $(v)$ then follows from the observation that the class of each triangle on $X$ is equal to $h^{3}$ and that $c^{2}=27 \mathfrak{o}_{F}$.

Now we introduce some correspondences on $F$. The most natural one is the incidence correspondence $I \subset F \times F$ which consists of pairs of lines meeting each other. Let $H_{1}$ and $H_{2}$ be two general hyperplanes sections on $X$. Then we define

$$
\begin{gathered}
\Gamma_{h}=\left\{\left(\left[l_{1}\right],\left[l_{2}\right]\right) \in F \times F: \exists x \in H_{1}, \text { such that } x \in l_{1} \cap l_{2}\right\} ; \\
\Gamma_{h^{2}}=\left\{\left(\left[l_{1}\right],\left[l_{2}\right]\right) \in F \times F: \exists x \in H_{1} \cap H_{2}, \text { such that } x \in l_{1} \cap l_{2}\right\} .
\end{gathered}
$$

Lemma A.4. We have the following list of identities.

(i) The cylinder homomorphism $\Psi$ satisfies

$$
\Psi(g)=6[X], \quad \Psi\left(g^{2}\right)=21 h, \quad \Psi\left(g^{3}\right)=36 h^{2}, \quad \Psi\left(g^{4}\right)=36 h^{3} .
$$

The Abel-Jacobi homomorphism $\Phi$ satisfies

$$
\Phi\left(h^{2}\right)=g, \quad \Phi\left(h^{3}\right)=g^{2}-c, \quad \Phi\left(h^{4}\right)=3 C_{x} .
$$

(ii) The action of $I$ on self-intersections of $g$ is given by

$$
I_{*}\left(g^{2}\right)=21[F], \quad I_{*}\left(g^{3}\right)=36 g, \quad I_{*}\left(g^{4}\right)=36 \Phi\left(h^{3}\right),
$$

where $I \subset F \times F$ is the incidence correspondence.

(iii) The action of $\Gamma_{h}$ on the self-intersections of $g$ is given by

$$
\left(\Gamma_{h}\right)^{*} g=6[F], \quad\left(\Gamma_{h}\right)^{*} g^{2}=21 g, \quad\left(\Gamma_{h}\right)^{*} g^{3}=36 \Phi\left(h^{3}\right), \quad\left(\Gamma_{h}\right)^{*} g^{4}=108 C_{x} .
$$


(iv) The action of $\Gamma_{h^{2}}$ on the self-intersections of $g$ is given by

$$
\left(\Gamma_{h^{2}}\right)^{*} g=6 g, \quad\left(\Gamma_{h^{2}}\right)^{*} g^{2}=21 \Phi\left(h^{3}\right), \quad\left(\Gamma_{h^{2}}\right)^{*} g^{3}=108 C_{x}, \quad\left(\Gamma_{h^{2}}\right)^{*} g^{4}=0 .
$$

Proof. First, for dimension reasons, we have $\Psi(g)=n_{1}[X]$ for some integer $n_{1}$. Then one computes

$$
n_{1} \equiv x \cdot \Psi(g) \equiv \Phi(x) \cdot g=C_{x} \cdot g \equiv 6 .
$$

Since $\Psi\left(g^{2}\right)$ lives on $X$, it has to be a multiple of $h$. We assume that $\Psi\left(g^{2}\right)=n_{2} h$, then we have

$$
n_{2} \equiv \Psi\left(g^{2}\right) \cdot l \equiv g^{2} \cdot \Phi(l)=g^{2} \cdot S_{l} \equiv 21 .
$$

In the last step, we used the fact that the cohomology class of $S_{l}$ is $\frac{1}{3}\left(g^{2}-c\right)$; see [52, §0]. Similarly, we have $\Psi\left(g^{3}\right)=n_{3} h^{2}$ and we determine $n_{3}=36$ by

$$
3 n_{3} \equiv \Psi\left(g^{3}\right) \cdot h^{2} \equiv g^{3} \cdot \Phi\left(h^{2}\right)=g^{3} \cdot g \equiv 108 .
$$

By Lemma A.3, we have $\Psi\left(\mathfrak{o}_{F}\right)=\frac{1}{3} h^{3}$. Hence $\Psi\left(g^{4}\right)=\Psi\left(108 \mathfrak{o}_{F}\right)=36 h^{3}$. The equality $g=\Phi\left(h^{2}\right)$ and $\Phi\left(h^{4}\right)=3 C_{x}$ are easy. To prove the remaining equality, we note that $P=\mathbb{P}\left(\mathscr{E}_{2}\right)$ and $q^{*} h$ is the Chern class of the relative $\mathcal{O}(1)$ bundle. Hence we have the equality

$$
q^{*} h^{2}+p^{*} c_{1}\left(\mathscr{E}_{2}\right) q^{*} h+p^{*} c_{2}\left(\mathscr{E}_{2}\right)=0 .
$$

Then it follows

$$
\begin{aligned}
q^{*} h^{3} & =q^{*} h\left(-p^{*} c_{1}\left(\mathscr{E}_{2}\right) q^{*} h-p^{*} c_{2}\left(\mathscr{E}_{2}\right)\right) \\
& =q^{*} h\left(p^{*} g q^{*} h-p^{*} c\right) \\
& =p^{*} g\left(p^{*} g q^{*} h-p^{*} c\right)-q^{*} h p^{*} c \\
& =p^{*}\left(g^{2}-c\right) q^{*} h-p^{*}\left(g^{2} c\right) .
\end{aligned}
$$

Hence $\Phi\left(h^{3}\right)=p_{*} q^{*} h^{3}=g^{2}-c$. This proves (i). Since $I_{*}=\Phi \circ \Psi,\left(\Gamma_{h}\right)^{*} g^{i}=\Phi\left(h \cdot \Psi\left(g^{i}\right)\right)$ and $\left(\Gamma_{h^{2}}\right)^{*} g^{i}=\Phi\left(h^{2} \cdot \Psi\left(g^{i}\right)\right)$, all the remaining identities follow from $(i)$.

Lemma A.5. $I_{*} \mathfrak{o}_{F}=\frac{1}{3}\left(g^{2}-c\right)$.

Proof. By $(v)$ of Lemma A.3, we see that $\Psi\left(\mathfrak{o}_{F}\right)=\frac{1}{3} h^{3}$. Then by (i) of Lemma A.4, we conclude the proof.

Proposition A.6. The following identities hold true in the Chow groups of $F \times F$

$$
\begin{aligned}
\Gamma_{h} & =\frac{1}{3}\left(g_{1}^{3}+g_{1}^{2} g_{2}+g_{1} g_{2}^{2}+g_{2}^{3}-2 g_{1} c_{1}-g_{1} c_{2}-g_{2} c_{1}-2 g_{2} c_{2}\right) ; \\
\Gamma_{h^{2}} & =\frac{1}{3}\left(g_{1}^{3} g_{2}+g_{1}^{2} g_{2}^{2}+g_{1} g_{2}^{3}-g_{1}^{2} c_{2}-2 g_{1} g_{2} c_{2}-g_{2}^{2} c_{1}-2 g_{1} g_{2} c_{1}+c_{1} c_{2}\right) .
\end{aligned}
$$

Proof. Let $h_{i}=p_{i}^{*} h$ on $X \times X$. In the proof of [54, Proposition 3.3], Voisin shows that there is a Künneth type decomposition

$$
\Delta_{X}=\Delta_{1}+\Delta_{0}
$$

in $\mathrm{CH}^{4}(X \times X)$ such that $\Delta_{1}=\sum_{i=0}^{4} \alpha_{i} h_{1}^{i} h_{2}^{4-i}$ and $\Delta_{0} \cdot h_{i}=0$ in $\mathrm{CH}^{5}(X \times X)$. One can show that $\alpha_{i}=\frac{1}{3}, i=0, \ldots, 4$, as follows. Let both side act on $h^{j}, j=1, \ldots, 4$, and get

$$
h^{j}=\alpha_{4-j}\left(h^{4}\right) h^{j}=3 \alpha_{4-j} h^{j} .
$$

Hence we get $\alpha_{i}=\frac{1}{3}, i=0, \ldots, 3$. By symmetry, we also have $\alpha_{4}=\frac{1}{3}$. Therefore we have

$$
\begin{aligned}
\Gamma_{h} & =(p \times p)_{*}(q \times q)^{*}\left(\Delta_{X} \cdot h_{1}\right) \\
& =\frac{1}{3}(p \times p)(q \times q)^{*}\left(h_{1} h_{2}^{4}+h_{1}^{2} h_{2}^{3}+h_{1}^{3} h_{2}^{2}+h_{1}^{4} h_{2}\right) \\
& =\frac{1}{3} \sum_{i=1}^{4} p_{1}^{*} \Phi\left(h^{i}\right) p_{2}^{*} \Phi\left(h^{5-i}\right) \\
& =\frac{1}{3}\left(3 p_{2}^{*} C_{x}+g_{1}\left(g_{2}^{2}-c_{2}\right)+\left(g_{1}^{2}-c_{1}\right) g_{2}+3 p_{1}^{*} C_{x}\right)
\end{aligned}
$$


Note that in the Proof of [53, Lemma 3.2], Voisin shows that $3 C_{x}=g^{3}-2 g c$. Put this into the above formula, we get the equality for $\Gamma_{h}$. The second equality can be proved in a similar way.

A.2. Secant lines and their specializations. We recall some definitions from [46]. Let $C_{1}, C_{2} \subset X$ be two disjoint curves on $X$.

Definition A.7. A secant line of the pair $\left(C_{1}, C_{2}\right)$ is a line on $X$ that meets both $C_{1}$ and $C_{2}$.

It is shown in [46] that the total number, if finite, of secant lines (counted with multiplicity) of $\left(C_{1}, C_{2}\right)$ is equal to $5 d_{1} d_{2}$, where $d_{i}=\operatorname{deg}\left(C_{i}\right), i=1,2$. We will be mainly interested in the case when both $C_{1}$ and $C_{2}$ are lines.

Lemma A.8. Let $l_{1}, l_{2}$ be two lines on $X$ meeting each other in a point $x$ and $\Pi=\mathbb{P}^{2}$ the plane spanned by $l_{1}$ and $l_{2}$. Assume that $\Pi$ is not contained in $X$ and $\Pi \cdot X=l_{1}+l_{2}+l_{3}$ for a third line $l_{3} \subset X$. Let $\left\{l_{t}: t \in T\right\}$ be a 1-dimensional family of lines on $X$ with $l_{t_{0}}=l_{1}$. Assume that for a general point $t \in T$, the pair $\left(l_{2}, l_{t}\right)$ has five secant lines $\left\{E_{1, t}, \ldots, E_{5, t}\right\}$. Then as $t$ specializes to $t_{0}$, four of the secant lines, say $\left\{E_{1, t}, \ldots, E_{4, t}\right\}$, specialize to four lines $\left\{E_{1}, \ldots, E_{4}\right\}$ passing through $x$ while the fifth secant line $E_{5, t}$ specializes to the line $l_{3}$, such that

$$
\left[l_{1}\right]+\left[l_{2}\right]+\left[E_{1}\right]+\left[E_{2}\right]+\left[E_{3}\right]+\left[E_{4}\right]=6 \mathfrak{o}_{F} .
$$

Proof. Note that the tangent space of $T$ at the point $t_{0}$ corresponds to a global section $v \in \mathrm{H}^{0}\left(l_{1}, \mathscr{N}_{l_{1} / X}\right)$. We may assume that $v(x) \neq 0$ and hence $v(x), \mathscr{T}_{l_{1}, x}$ and $\mathscr{T}_{l_{2}, x}$ span a linear $\mathbb{P}^{3}$ which is tangent to $X$ at the point $x$. There are 6 lines on $X$ passing through $x$ and contained in the above $\mathbb{P}^{3}$. These lines are $\left\{l_{1}, l_{2}, E_{1}, \ldots, E_{4}\right\}$. By construction, one easily checks that the cycle $\left[l_{1}\right]+\left[l_{2}\right]+\left[E_{1}\right]+\ldots+\left[E_{4}\right]$ represents $C_{x} \cdot g=6 \mathfrak{o}_{F}$.

A.3. The geometry of lines of second type. If $l \subset X$ is a line contained in $X$, then either

$$
\mathscr{N}_{l / X} \cong \mathcal{O}(1) \oplus \mathcal{O}^{2}
$$

in which case $l$ is said to be of first type, or

$$
\mathscr{N}_{l / X} \cong \mathcal{O}(1)^{2} \oplus \mathcal{O}(-1)
$$

in which case $l$ is said to be of second type. It is known that for a general point $[l] \in F$, the corresponding line $l$ is of first type. Let $\Sigma_{2} \subset F$ be the closed subscheme of lines of second type.

Lemma A.9 $([19,2]), \Sigma_{2}$ is a surface on $F$ whose cycle class is $5\left(g^{2}-c\right)$.

Proof. The fact that $\Sigma_{2}$ is a surface is proved in [19, Corollary 7.6]. The cohomology class of $\Sigma_{2}$ is computed in [2] and is actually valid at the level of Chow groups.

Proposition A.10. For all $\sigma \in \mathrm{CH}^{2}(F)_{\mathrm{hom}},\left[\Sigma_{2}\right] \cdot \sigma=5 g^{2} \cdot \sigma$.

Proof. Let $\sigma \in \mathrm{CH}^{2}(F)_{\text {hom }}$, then by (iii) of Lemma A.3, we have $c \cdot \sigma=0$. The proposition then follows from Lemma A.9.

Now we study the geometry of lines of second type. Let $l \subset X$ be a line of second type. Then there exist a linear $\mathbb{P}^{3}$, denoted $\mathbb{P}_{\langle l\rangle}^{3}$, containing $l$ which is tangent to $X$ along $l$. Let $B \cong \mathbb{P}^{1}$ be the rational curve parameterizing all planes containing $l$ and contained in the linear $\mathbb{P}_{\langle l\rangle}^{3}$. For $t \in B$, we use $\Pi_{t}$ to denote the corresponding plane. When $t$ is general, then $\Pi_{t}$ is not contained in $X$ and hence we have

$$
\Pi_{t} \cdot X=2 l+l_{t},
$$

for some line $l_{t}$. As $t$ moves in $B$, the points $\left[l_{t}\right]$ traces out a curve $\mathbb{P}^{1} \cong \mathcal{E}_{[l]} \subset F$. The lines parameterized by $\mathcal{E}_{[l]}$ swipe out a cubic surface singular along $l$, which is simply $\mathbb{P}_{\langle l\rangle}^{3} \cap X$.

Proposition A.11. Let $l \subset X$ be a line of second type and notation as above. Then there is a unique line $l^{\prime} \subset X$, disjoint with $l$, together with a natural isomorphism $\alpha_{1}: \mathcal{E}_{[l]} \rightarrow l^{\prime}$ and a degree 2 morphism $\alpha_{2}: \mathcal{E}_{[l]} \rightarrow l$ such that the following holds : for all point $s \in \mathcal{E}_{[l]}$, the corresponding line $l_{s} \subset X$ is the line connecting the points $\alpha_{1}(s)$ and $\alpha_{2}(s)$. The surface $S$ is smooth away from $l$. 
Proof. This is the consequence of the geometry of cubic surfaces singular along a curve. Let $S=\mathbb{P}_{\langle l\rangle}^{3} \cap X$ be the cubic surface swept out by all lines parameterized by $\mathcal{E}_{[l]}$. Then $S$ is singular along $l$. Take homogeneous coordinates $\left[X_{0}: X_{1}: X_{2}: X_{3}\right]$ on $\mathbb{P}_{\langle l\rangle}^{3}$ such that $l$ is defined by $X_{2}=X_{3}=0$. Let $l^{\prime}$ be the line defined by $X_{2}=X_{3}=0$. We easily see that $S \subset \mathbb{P}_{\langle l\rangle}^{3}$ is defined by an equation

$$
X_{0} Q_{1}\left(X_{2}, X_{3}\right)-X_{1} Q_{0}\left(X_{2}, X_{3}\right)=0
$$

where $Q_{0}, Q_{1}$ are homogeneous polynomials of degree 2 in $X_{2}, X_{3}$. We see that the smoothness of $X$ forces $Q_{0}$ and $Q_{1}$ to be linearly independent. Indeed, if $Q_{0}$ and $Q_{1}$ are proportional to each other, then we can apply a linear change of coordinates and the equation for $S$ in $\mathbb{P}_{\langle l\rangle}^{3}$ can be written as $X_{0} X_{2} X_{3}=0$ or $X_{0} X_{2}^{2}=0$. In either case, one easily checks that $X$ is singular at the point $[0: 1: 0: 0] \in \mathbb{P}_{\langle l\rangle}^{3} \cdot \mathrm{We}$ use $\left[X_{0}: X_{1}\right]$ as homogeneous coordinates on $l$. Pick a point $t=\left[t_{0}: t_{1}\right] \in l$. A line on $S$ passing through $t$ can be parameterized by

$$
\left[\lambda_{0}: \lambda_{1}\right] \mapsto\left[\lambda_{0} t_{0}: \lambda_{0} t_{1}: \lambda_{1} a: \lambda_{2} b\right]
$$

where $t_{0} Q_{1}(a, b)-t_{1} Q_{0}(a, b)=0$. We note that any such line always meets the line $l^{\prime}$ at the point $[0: 0: a: b]$. For a fixed $t$, we have two pairs (counted with multiplicities) $(a, b)$ satisfying the above equation. Consequently through a general point $t \in l$, there are two points $s_{1}, s_{2} \in \mathcal{E}_{[l]}$ such that both $l_{s_{1}}$ and $l_{s_{2}}$ pass through $t$. Then we take $\alpha_{1}(s)=l_{s} \cap l^{\prime}$ and $\alpha_{2}(s)=l_{s} \cap l$ for all $s \in \mathcal{E}_{[l]}$. We still need to show that $S$ is smooth away from $l$. Note that the above description of $S$ implies that $S$ is smooth away from $l$ and $l^{\prime}$. However the fact that $\alpha_{1}$ is an isomorphism forces $S$ to be smooth along $l^{\prime}$.

Proposition A.12. Let $l \subset X$ be a line of second type and $s_{1}, s_{2} \in \mathcal{E}_{[l]}$ two points such that the corresponding lines $l_{1}=l_{s_{1}}$ and $l_{2}=l_{s_{2}}$ are disjoint. Then the set of secant lines of $\left(l_{1}, l_{2}\right)$ is given by $4 l+l^{\prime}$, where $l^{\prime}$ is the line as in Proposition A.11.

Proof. Any secant line $L$ of $\left(l_{1}, l_{2}\right)$ different from $l$ or $l^{\prime}$ is in the smooth locus of $S$. This implies that $\mathscr{N}_{l / S} \cong \mathcal{O}(-1)$. It follows that $L$ does not move on $S$. Thus $\left(l_{1}, l_{2}\right)$ has finitely many, and hence five, secant lines. Note that both $l_{1}$ and $l_{2}$ are pointing the the positive direction of $\mathscr{N}_{l / X}$. Hence the multiplicity of $l$, as a secant line of $\left(l_{1}, l_{2}\right)$ is 4 ; see $[46, \S 3]$. Hence the multiplicity of $l^{\prime}$ has to be 1 . This finishes the proof.

\section{Appendix B. Rational maps and Chow groups}

We consider $\varphi: X \rightarrow Y$ a rational map between projective varieties defined over a field $k$ of characteristic zero. Let

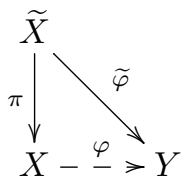

be a resolution of $\varphi$, that is, $\pi: \widetilde{X} \rightarrow X$ is a projective birational morphism such that $\varphi$ extends to a morphism $\widetilde{\varphi}: \widetilde{X} \rightarrow Y$. Note that no smoothness assumption is required on $\widetilde{X}$.

Assume that $X$ is smooth. Then we define a map $\varphi_{*}: \mathrm{CH}_{l}(X) \rightarrow \mathrm{CH}_{l}(Y)$ by the formula

$$
\varphi_{*} \alpha:=\widetilde{\varphi}_{*} \pi^{*} \alpha \quad \text { for all } \alpha \in \mathrm{CH}_{l}(X) .
$$

Here, $\pi^{*}: \mathrm{CH}_{l}(X) \rightarrow \mathrm{CH}_{l}(\tilde{X})$ is the pull-back map as defined in $[25, \S 8]$.

Lemma B.1. Assume that $X$ is smooth. The map $\varphi_{*}: \mathrm{CH}_{l}(X) \rightarrow \mathrm{CH}_{l}(Y)$ defined above does not depend on a choice of resolution for $\varphi$.

Proof. Let $\pi_{1}: X_{1} \rightarrow X, \varphi_{1}: X_{1} \rightarrow Y$ be a resolution of $\varphi$, and let $\pi_{2}: X_{2} \rightarrow X, \varphi_{2}: X_{2} \rightarrow Y$ be another resolution of $\varphi$. There is then a projective variety $\widetilde{X}$ and birational morphisms $\widetilde{\pi}_{1}: \widetilde{X} \rightarrow X_{1}$ and $\tilde{\pi}_{2}: \widetilde{X} \rightarrow X_{2}$ such that both $\varphi_{1}$ and $\varphi_{2}$ extend to a morphism $\widetilde{\varphi}: \widetilde{X} \rightarrow Y$. It is then enough to see that

$$
\widetilde{\varphi}_{*}\left(\pi_{1} \circ \tilde{\pi}_{1}\right)^{*}=\left(\varphi_{1}\right)_{*} \pi_{1}^{*}: \mathrm{CH}_{l}(X) \rightarrow \mathrm{CH}_{l}(Y) \text {. }
$$


The identity $\varphi_{1} \circ \widetilde{\pi}_{1}=\widetilde{\varphi}$ gives $\widetilde{\varphi}_{*}\left(\pi_{1} \circ \widetilde{\pi}_{1}\right)^{*}=\left(\varphi_{1}\right)_{*}\left(\widetilde{\pi}_{1}\right)_{*}\left(\pi_{1} \circ \widetilde{\pi}_{1}\right)^{*}$. We may then conclude by the projection formula $\left[25\right.$, Proposition 8.1.1] which implies that $\left(\widetilde{\pi}_{1}\right)_{*}\left(\pi_{1} \circ \widetilde{\pi}_{1}\right)^{*}=\pi_{1}^{*}$.

If $Y$, instead of $X$, is assumed to be smooth, then we define a map $\varphi^{*}: \mathrm{CH}^{l}(Y) \rightarrow \mathrm{CH}^{l}(X)$ by the formula

$$
\varphi^{*} \beta:=\pi_{*} \widetilde{\varphi}^{*} \beta \quad \text { for all } \beta \in \mathrm{CH}^{l}(Y) .
$$

Likewise, we have

Lemma B.2. Assume that $Y$ is smooth. The map $\varphi^{*}: \mathrm{CH}^{l}(Y) \rightarrow \mathrm{CH}^{l}(X)$ defined above does not depend on a choice of resolution for $\varphi$.

Let now $p_{X}: X \times Y \rightarrow X$ and $p_{Y}: X \times Y \rightarrow Y$ be the natural projections, and let $\Gamma_{\varphi} \subset X \times Y$ be the closure of the graph of $\varphi$, given with the natural projections

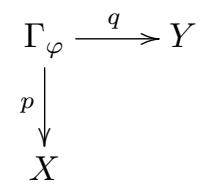

The morphism $p$ is birational and the morphism $q$ clearly extends $\varphi$. Therefore, as a consequence of Lemmas B.1 and B.2, we get

Lemma B.3. If $X$ is smooth, then $\varphi_{*} \alpha=q_{*} p^{*} \alpha$ for all $\alpha \in \mathrm{CH}_{l}(X)$. If $Y$ is smooth, then $\varphi^{*} \beta=p_{*} q^{*} \beta$ for all $\beta \in \mathrm{CH}^{l}(Y)$. If $X$ and $Y$ are both smooth, then

$$
\varphi_{*} \alpha=\left(\Gamma_{\varphi}\right)_{*} \alpha:=\left(p_{Y}\right)_{*}\left(\Gamma_{\varphi} \cdot p_{X}^{*} \alpha\right) \quad \text { and } \quad \varphi^{*} \beta=\left(\Gamma_{\varphi}\right)^{*} \beta:=\left(p_{X}\right)_{*}\left(\Gamma_{\varphi} \cdot p_{Y}^{*} \beta\right) .
$$

We now want to understand, when $X$ and $Y$ are both smooth, to which extent $\varphi^{*}: \mathrm{CH}^{*}(Y) \rightarrow \mathrm{CH}^{*}(X)$ is compatible with intersection product.

Lemma B.4. Let $\pi: \widetilde{X} \rightarrow X$ be a dominant morphism between smooth projective varieties and let $x$ and $y$ be two cycles in $\mathrm{CH}_{*}(\widetilde{X})$. Then

$$
\pi_{*}(x \cdot y)=\pi_{*} x \cdot \pi_{*} y+\pi_{*}\left(\left(x-\pi^{*} \pi_{*} x\right) \cdot\left(y-\pi^{*} \pi_{*} y\right)\right) .
$$

Proof. Let's define $x^{\prime}:=x-\pi^{*} \pi_{*} x$ and $y^{\prime}:=y-\pi^{*} \pi_{*} y$. By the projection formula, $\pi_{*} \pi^{*}$ acts as the identity on $\mathrm{CH}_{*}(X)$. Therefore $\pi_{*} x^{\prime}=0$ and $\pi_{*} y^{\prime}=0$. The projection formula gives $\pi_{*}\left(\left(\pi^{*} \pi_{*} x\right) \cdot y^{\prime}\right)=$ $\pi_{*} x \cdot \pi_{*} y^{\prime}=0$ and $\pi_{*}\left(x^{\prime} \cdot\left(\pi^{*} \pi_{*} y\right)\right)=\pi_{*} x^{\prime} \cdot \pi_{*} y=0$. This yields

$$
\pi_{*}(x \cdot y)=\pi_{*}\left(\pi^{*} \pi_{*} x \cdot \pi^{*} \pi_{*} y\right)+\pi_{*}\left(x^{\prime} \cdot y^{\prime}\right)=\pi_{*} x \cdot \pi_{*} y+\pi_{*}\left(x^{\prime} \cdot y^{\prime}\right) .
$$

For a smooth projective variety $X$ over $k$, we write

$$
T^{2}(X):=\operatorname{ker}\left\{A J^{2}: \mathrm{CH}^{2}(X)_{\text {hom }} \rightarrow J^{2}(X)\right\}
$$

for the kernel of Griffiths' second Abel-Jacobi map to the second intermediate Jacobian $J^{2}(X)$ which is a quotient of $H^{3}(X, \mathbb{C})$. The group $T^{2}(X)$ is a birational invariant of smooth projective varieties. Precisely, we have

Lemma B.5. Let $\pi: \widetilde{X} \rightarrow X$ be a birational map between smooth projective varieties. Then $\pi^{*} \pi_{*}$ acts as the identity on $\mathrm{CH}_{0}(\widetilde{X}), \operatorname{Griff}_{1}(\widetilde{X}), \operatorname{Griff}^{2}(\widetilde{X}), T^{2}(\widetilde{X}), \mathrm{CH}^{1}(\widetilde{X})_{\text {hom }}$ and $\mathrm{CH}^{0}(\widetilde{X})$.

Proof. This is proved in [50, Proposition 5.4] in the case when $\pi$ is a birational morphism. In the general case, by resolution of singularities, there is a smooth projective variety $Y$ and birational morphisms $f: Y \rightarrow \widetilde{X}$ and $\widetilde{\pi}: \widetilde{X} \rightarrow X$ such that $\widetilde{\pi}=\pi \circ f$. By definition of the action of rational maps on Chow groups, we have $\pi^{*} \pi_{*}=f_{*} \widetilde{\pi}^{*} \widetilde{\pi}_{*} f^{*}$ and the proof of the lemma reduces to the case of birational morphisms. 
Proposition B.6. Let $\varphi: X \rightarrow Y$ be a rational map between smooth projective varieties and let $d$ be the dimension of $X$. Let $\alpha \in \mathrm{CH}^{*}(Y)$ be a cycle that belongs to one of the sub-groups $\mathrm{CH}^{d}(Y), T^{2}(Y)$, $C H^{1}(Y)$ hom or $\mathrm{CH}^{0}(Y)$ (respectively $\alpha \in \operatorname{Griff}^{d-1}(Y)$ or $\operatorname{Griff}^{2}(Y)$ ), and let $\beta \in \mathrm{CH}^{*}(Y)$ be any cycle. Then

$$
\left.\varphi^{*}(\alpha \cdot \beta)=\varphi^{*} \alpha \cdot \varphi^{*} \beta \in \mathrm{CH}^{*}(X) \text { (resp. } \in \operatorname{Griff}^{*}(X)\right) .
$$

Proof. Let $\pi: \widetilde{X} \rightarrow X, \widetilde{\varphi}: \widetilde{X} \rightarrow Y$ be a resolution of $\varphi$ with $\widetilde{X}$ smooth. For $x$ belonging to one of the groups $\mathrm{CH}_{0}(\widetilde{X}), \operatorname{Griff}_{1}(\widetilde{X}), \operatorname{Griff}^{2}(\widetilde{X}), T^{2}(\widetilde{X}), C H^{1}(\widetilde{X})_{\text {hom }}$ or $\mathrm{CH}^{0}(\widetilde{X})$, we have by Lemma B.5 $x=\pi^{*} \pi_{*} x$, so that Lemma B.4 yields

$$
\pi_{*}(x \cdot y)=\pi_{*} x \cdot \pi_{*} y
$$

If we now set $x=\widetilde{\varphi}^{*} \alpha$ for $\alpha$ as in the statement of the proposition, then $x$ defines a cycle as above (if $\alpha \in T^{2}(Y)$, then $x \in T^{2}(\widetilde{X})$ by functoriality of the Abel-Jacobi map with respect to the action of correspondences), and (121) gives :

$$
\begin{aligned}
\varphi^{*} \alpha \cdot \varphi^{*} \beta & =\pi_{*} \widetilde{\varphi}^{*} \alpha \cdot \pi_{*} \widetilde{\varphi}^{*} \beta \\
& =\pi_{*}\left(\widetilde{\varphi}^{*} \alpha \cdot \widetilde{\varphi}^{*} \beta\right) \\
& =\pi_{*} \widetilde{\varphi}^{*}(\alpha \cdot \beta) \\
& =\varphi^{*}(\alpha \cdot \beta) .
\end{aligned}
$$

There is also a projection formula for rational maps.

Proposition B.7. Let $\varphi: X \rightarrow Y$ be a rational map between smooth projective varieties and let $d$ be the dimension of $X$. Let $\alpha \in \mathrm{CH}^{*}(Y)$ be a cycle that belongs to one of the sub-groups $\mathrm{CH}^{d}(Y), T^{2}(Y)$, $C H^{1}(Y)_{\text {hom }}$ or $\mathrm{CH}^{0}(Y)$ (respectively $\alpha \in \operatorname{Griff}^{d-1}(Y)$ or $\operatorname{Griff}^{2}(Y)$ ), and let $\beta \in \mathrm{CH}^{*}(X)$ be any cycle. Then

$$
\varphi_{*}\left(\varphi^{*} \alpha \cdot \beta\right)=\alpha \cdot \varphi_{*} \beta \in \mathrm{CH}^{*}(Y)\left(\text { resp. } \in \operatorname{Griff}^{*}(Y)\right) .
$$

In particular, if $\varphi: X \rightarrow Y$ is a finite rational map of degree $\operatorname{deg} \varphi$, then

$$
\varphi_{*} \varphi^{*} \alpha=(\operatorname{deg} \varphi) \alpha \in \mathrm{CH}^{*}(Y)\left(\text { resp. } \in \operatorname{Griff}^{*}(Y)\right) .
$$

Proof. Again consider a resolution of $\varphi$ as in the proof of Proposition B.6. By Lemmas B.1 and B.2, Lemma B.5 and the projection formula for morphism of smooth projective varieties, we have

$$
\begin{aligned}
\varphi_{*}\left(\varphi^{*} \alpha \cdot \beta\right) & =\widetilde{\varphi}_{*} \pi^{*}\left(\pi_{*} \widetilde{\varphi}^{*} \alpha \cdot \beta\right) \\
& =\widetilde{\varphi}_{*}\left(\pi^{*} \pi_{*} \widetilde{\varphi}^{*} \alpha \cdot \pi^{*} \beta\right) \\
& =\widetilde{\varphi}_{*}\left(\widetilde{\varphi}^{*} \alpha \cdot \pi^{*} \beta\right) \\
& =\alpha \cdot \widetilde{\varphi}_{*} \pi^{*} \beta \\
& =\alpha \cdot \varphi_{*} \beta .
\end{aligned}
$$

The last statement follows by taking $\beta=[X] \in \mathrm{CH}^{0}(X)$. 


\section{REFERENCES}

[1] A. Altman and S. Kleiman, Foundations of the theory of Fano schemes, Compositio Mathematica 34, no. 1 (1977), p. 3-47.

[2] E. Amerik, A computation of invariants of a rational self-map, Ann. Fac. Sci. Toulouse Math. 18 (2009), no. 3, 445-457.

[3] E. Amerik and C. Voisin, Potential density of rational points on the variety of lines of a cubic fourfold, Duke Math. J. 145(2008), no. 2, 379-408.

[4] G. Ancona, Décomposition de motifs abéliens, preprint, 2013.

[5] Y. André and B. Kahn, Nilpotence, radicaux et structures monodales, with an appendix by Peter O'Sullivan, Rend. Sem. Mat. Univ. Padova 108 (2002), 107-291.

[6] A. Beauville, Variétes Kähleriennes dont la premiére classe de Chern est nulle, J. Diff. Geom. 18 (1983), 755-782.

[7] A. Beauville, Quelques remarques sur la transformation de Fourier dans l'anneau de Chow d'une variété abélienne, Algebraic Geometry (Tokyo/Kyoto 1982), LN 1016, 238-260.

[8] A. Beauville, Sur l'anneau de Chow d'une variété abélienne. Math. Ann. 273, 647-651 (1986).

[9] A. Beauville, On the splitting of the Bloch-Beilinson filtration, Algebraic cycles and motives (vol. 2), London Math. Soc. Lecture Notes 344, 38-53 ; Cambridge University Press (2007).

[10] A. Beauville and R. Donagi, La variété des droites d'une hypersurface cubique de dimension 4, C. R. Acad. Sci. Paris Sr. I Math. 301 (1985), no. 14, 703-706.

[11] A. Beauville and C. Voisin, On the Chow ring of a K3 surface. J. Algebraic Geom. 13 (2004), 417-426.

[12] A. Beilinson, Height pairing between algebraic cycles, K-theory, arithmetic and geometry (Moscow, 1984-1986), 1-25, Lecture Notes in Math., 1289, Springer, Berlin, 1987.

[13] S. Bloch, Lectures on algebraic cycles, Second edition. New Mathematical Monographs, Cambridge University Press, Cambridge, 2010.

[14] S. Bloch and V. Srinivas, Remarks on correspondences and algebraic cycles, Amer. J. Math. 105 (1983), no. 5, $1235-1253$.

[15] F. Bogomolov, Hamiltonian Kählerian manifolds, Dokl. Akad. Nauk SSSR 243 (1978), no. 5, 1101-1104.

[16] F. Bogomolov, On the cohomology ring of simple hyperkähler manifold (on the results of Verbitsky), Geometric and Functional Analysis, Vol. 6 No. 4 (1996), 612-618.

[17] A. Căldăraru, Derived categories of twisted sheaves on Calabi-Yau manifolds, Ph.D. thesis, Cornell University, 2000.

[18] M.A. de Cataldo and L. Migliorini, The Chow groups and the motive of the Hilbert scheme of points on a surface, J. Algebra 251 (2002), no. 2, 824-848.

[19] C.H. Clemens and P.A. Griffiths, The intermediate Jacobian of the cubic threefold, Ann. of Math. 95 (1972), 281-356.

[20] I. Coskun and J. Starr, Rational curves on smooth cubic hypersurfaces, Int. Math. Res. Not., 2009, no.24, 4626-4641.

[21] C. Deninger and J. Murre, Motivic decomposition of abelian schemes and the Fourier transform, J. Reine Angew. Math. 422 (1991), 201-219.

[22] L. Fu, Decomposition of small diagonals and Chow rings of hypersurfaces and Calabi-Yau complete intersections, Adv. Math. 244 (2013), 894-924.

[23] L. Fu, On the action of symplectic automorphisms on the $\mathrm{CH}_{0}$-groups of some hyper-Kähler fourfolds, preprint, 2013.

[24] A. Fujiki, On the de Rham cohomology group of a compact Kähler symplectic manifold, Adv. Studies in Pure Math. 10, Algebraic Geometry, Sendai 1985, 105-165, 1987.

[25] W. Fulton, Intersection theory, Second edition. Ergebnisse der Mathematik und ihrer Grenzgebiete. 3. Folge. A Series of Modern Surveys in Mathematics, Springer-Verlag, 1998.

[26] U. Greiner, On the Chow ring of birational irreducible symplectic varieties, preprint, 2013.

[27] B.H. Gross and C. Schoen, The modified diagonal cycle on the triple product of a pointed curve, Ann. Inst. Fourier (Grenoble) 45 (1995), no. 3, 649-679.

[28] D. Huybrechts, Birational symplectic manifolds and their deformations, J. Differential Geom. 45 (1997), $488-513$.

[29] D. Huybrechts, The Kähler cone of a compact hyperkähler manifold, Math. Ann. 326 (2003), no. 3, 499-513.

[30] D. Huybrechts, Symplectic automorphisms of K3 surfaces of arbitrary order, Math. Res. Lett. 19 (2012), $947-951$.

[31] D. Huybrechts and M. Lehn, The geometry of moduli spaces of sheaves, second edition, Cambridge University Press, 2010.

[32] U. Jannsen, Motivic sheaves and filtrations on Chow groups, In Motives (Seattle, WA, 1991), volume 55 of Proc. Sympos. Pure Math., pages 245-302. Amer. Math. Soc., Providence, RI, 1994.

[33] S.I. Kimura, Chow groups are finite dimensional, in some sense, Math. Ann. 331 (2005), no. 1, 173-201.

[34] Y. Manin, Correspondences, motifs and monoidal transformations, Mat. Sb. (N.S.) 77 (119) (1968) $475-507$.

[35] E. Markman, The Beauville-Bogomolov class as a characteristic class, preprint, 2011.

[36] B. Moonen, On the Chow motive of an abelian scheme with non-trivial endomorphisms, J. Reine Angew. Math., in press.

[37] B. Moonen and Q. Yin, On a question of O'Grady about modified diagonals, preprint, 2013.

[38] J. Murre, On the motive of an algebraic surface, J. Reine Angew. Math. 409 (1990), 190-204.

[39] J. Murre, On a conjectural filtration on the Chow groups of an algebraic variety. I. The general conjectures and some examples, Indag. Math. 4 (1993), no. 2, 177-188.

[40] K. O'Grady, The weight-two Hodge structure of moduli spaces of sheaves on a K3 surface, J. Alg. Geom. 6 (1997), 599-644. 
[41] K. O'Grady, Computations with modified diagonals, preprint, 2013.

[42] P. O'Sullivan, The structure of certain rigid tensor categories, C. R. Math. Acad. Sci. Paris 340 (2005), no. 8 , $557-562$.

[43] P. O'Sullivan, Algebraic cycles on an abelian variety, J. Reine Angew. Math. 654 (2011), 1-81.

[44] A. Rapagnetta, On the Beauville form of the known irreducible symplectic varieties, Math. Ann. 340 (2008), 77-95.

[45] A. Rojtman, The torsion of the group of 0-cycles modulo rational equivalence, Ann. of Math. 111 (1980), no. 3, $553-569$.

[46] M. Shen, On relations among 1-cycles on cubic hypersurfaces, J. Alg. Geom., to appear.

[47] M. Shen, Surface with involution and Prym constructions, preprint, 2012.

[48] M. Shen and C. Vial, On modified diagonals, in preparation.

[49] C. Vial, Projectors on the intermediate algebraic Jacobians, New York J. Math. 19 (2013), 793-822.

[50] C. Vial, Algebraic cycles and fibrations, Doc. Math. 18 (2013) 1521-1553.

[51] C. Vial, Remarks on motives of abelian type, preprint, 2012.

[52] C. Voisin, Théorème de Torelli pour les cubiques de $\mathbb{P}^{5}$, Invent. Math. 86 (1986), no. 3, 577-601.

[53] C. Voisin, Intrinsic pseudo-volume forms and K-correspondences, The Fano Conference, (Eds A.Collino, A. Conte, M. Marchisio), 761-792, Univ. Torino, Turin, (2004).

[54] C. Voisin, On the Chow ring of certain algebraic hyperkähler manifolds, Pure Appl. Math. Q. 4(2008), no. 3, part 2, 613-649.

[55] C. Voisin, Chow rings and decomposition theorems for families of K3 surfaces and Calabi-Yau hypersurfaces, Geometry \& Topology 16 (2012), 433-473.

[56] C. Voisin, Symplectic involutions of K3 surfaces act trivially on $\mathrm{CH}_{0}$, Doc. Math. 17 (2012), 851-860.

[57] C. Voisin, Chow rings, decomposition of the diagonal and the topology of families, Annals of Math. Studies 187, Princeton University Press 2014.

[58] J. Wierzba, Birational geometry of symplectic 4-folds, preprint, 2002.

DPMms, University of Cambridge, Wilberforce Road, Cambridge CB3 0WB, UK

E-mail address: M.Shen@dpmms.cam.ac.uk, C.Vial@dpmms.cam.ac.uk 